
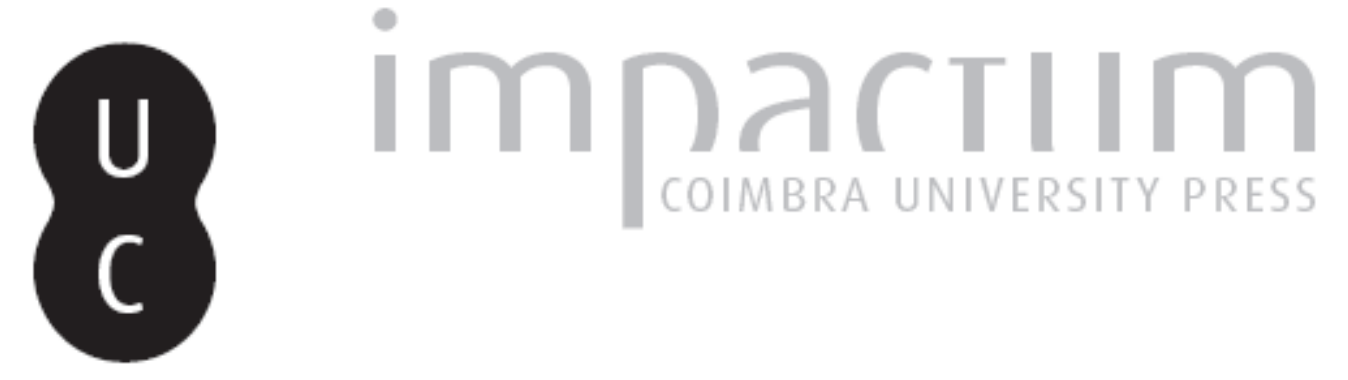

\title{
La mine antique d'Aljustrel (Portugal) et les tables de bronze de Vipasca
}

\author{
Autor(es): Domergue, Claude
}

Publicado por: Imprensa da Universidade de Coimbra

URL persistente:

URl:http://hdl.handle.net/10316.2/45696

DOI:

DOI:https://dx.doi.org/10.14195/1647-8657_22_1

Accessed : $\quad$ 26-Apr-2023 09:12:30

A navegação consulta e descarregamento dos títulos inseridos nas Bibliotecas Digitais UC Digitalis, UC Pombalina e UC Impactum, pressupõem a aceitação plena e sem reservas dos Termos e Condições de Uso destas Bibliotecas Digitais, disponíveis em https://digitalis.uc.pt/pt-pt/termos.

Conforme exposto nos referidos Termos e Condições de Uso, o descarregamento de títulos de acesso restrito requer uma licença válida de autorização devendo o utilizador aceder ao(s) documento(s) a partir de um endereço de IP da instituição detentora da supramencionada licença.

Ao utilizador é apenas permitido o descarregamento para uso pessoal, pelo que o emprego do(s) título(s) descarregado(s) para outro fim, designadamente comercial, carece de autorização do respetivo autor ou editor da obra.

Na medida em que todas as obras da UC Digitalis se encontram protegidas pelo Código do Direito de Autor e Direitos Conexos e demais legislação aplicável, toda a cópia, parcial ou total, deste documento, nos casos em que é legalmente admitida, deverá conter ou fazer-se acompanhar por este aviso.

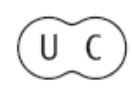


FACULDADE DE LETRAS

INSTITUTO DE ARQUEOLOGIA

\section{CONIMBRIGA}

VOLUMEXXII

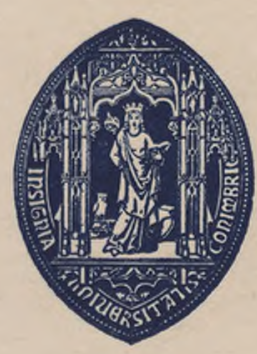

UNIVERSIDADE DE COIMBRA

1983 
Claude Domergue

Professor da Universidade de Toulouse

\author{
LA MINE ANTIQUE D'ALJUSTREL (PORTUGAL) ET LES TABLES \\ DE BRONZE DE VIPASCA \\ Conimbriga, XXII (1983), 5-193
}

RÉSUMÉ: La présente étude est divisée en deux parties. La première est consacrée à la mine d'Aljustrel. On s'est efforcé d'y rassembler tout ce que l'on sait de son exploitation. Sont ainsi examinés les conditions de gisement, la nature des minerais recherchés par les Anciens, les travaux miniers, les métaux produits, la chronologie.

La deuxième partie contient l'examen des deux tables en bronze trouvées respectivement en 1876 et en 1906 à Aljustrel, anciennement Vipasca. Chacun de ces deux textes (Vip. I et Vip. II) est traduit et commenté. Ils apparaissent l'un et l'autre comme étant des fragments de règlements miniers locaux, dont certaines dispositions ont un caractère général tandis que d'autres ont une portée plus réduite, quelques-unes même semblant limitées à la mine de Vipasca.

Resumo: O presente estudo divide-se em duas partes. A primeira, consagrada à mina antiga de Aljustrel, reúne tudo o que se conseguiu averiguar sobre a sua exploração. Examinam-se as condições da jazida, a natureza dos minérios que na Antiguidade se exploraram, os trabalhos de mineração, os metais que se produziram, a cronologia.

$\mathrm{Na}$ segunda parte estudam-se as duas tábuas de bronze encontradas em Aljustrel (antiga Vipasca), respectivamente em 1876 e 1906. Os dois textos (Vip. I e Vip. II) são traduzidos e comentados. Ambos se apresentam como fragmentos de regulamentos locais das minas; algumas disposições são de carácter geral; outras têm alcance mais reduzido ; algumas parece mesmo limitarem-se à mina de Vipasca. 
(Página deixada propositadamente em branco) 


\title{
LA MINE ANTIQUE D'ALJUSTREL (PORTUGAL) ET LES TABLES DE BRONZE DE VIPASCA
}

\author{
LIVRE PREMIER \\ CHAPITRE UNIQUE
}

LA MINE ANTIQUE D'ALJUSTREL

Avec S. Domingos (POR 7) et Caveira (POR 36), la mine d'AIjustrel $i^{1}$ ) se trouve dans la partie occidentale de la zone pyriteuse qui s'étend de la province de Séville (Espagne) jusqu'à l'Océan Atlantique. On retrouve donc, dans cette région du Portugal, des gisements analogues à ceux de la province de Huelva.

\section{A. Les gisements}

Ainsi, à Aljustrel, les terrains encaissants sont constitués par des schistes et des grauwackes d'une part, et par des porphyres de l'autre. Trois gisements principaux y dessinent un arc de cercle $\mathrm{du}$ nord-ouest au sud-est: aux extrémités, respectivement ceux de S. João do Deserto et des Algares, entre lesquels prend place celui de Moinho. Seuls les deux premiers nous intéressent, car, (i)

(i) Carte topographique du Portugal $\left(1 / 25000^{\mathrm{e}}\right)$, feuille 538 «Messejana», coordonnées Lambert 197,6-99,6 (Algares).

Les indications telles que POR 7 et POR 36 renvoient à notre Catalogue des mines et fonderies antiques de la Péninsule Ibérique (section «Portugal») d'où est extraite la notice concernant le site d'Aljustrel, qui forme la première partie de cette étude. Dans son premier état, la deuxième partie constituait un appendice à notre thèse sur Les mines de la Péninsule Ibérique à Vépoque romaine (à paraître prochainement). Dans son état actuel, elle a bénéficié des remarques des membres de notre jury, en particulier de M.M. J.Le Gall et W. Seston. D'autre part nous avons jugé que, pour être publiée à part, elle devait être refondue et améliorée. 
par leurs affleurements, ils ont attiré l'attention des Anciens qui les ont travaillés; Moinho en revanche était aveugle $\left(^{2}\right)$ et n'a pas été touché par ces derniers.

\section{Gisement des Algares}

11 s'allonge sur $1 \mathrm{~km}$ et se compose de trois masses minéralisées, d'orientation générale $\mathrm{N} .140^{\circ} \mathrm{O}$, avec un pendage de 60 à $70^{\circ}$ à l'est: le filon du Toit ou filon Est, le filon du Centre et le filon $\mathrm{du} \operatorname{Mur}\left({ }^{3}\right)$. Les deux derniers constituent pratiquement un seul et même ensemble, signalé en surface par le puissant chapeau de fer du filon du Mur: $900 \mathrm{~m}$ de longueur, 10 à $35 \mathrm{~m}$ d'épaisseur, «crestón» de $15 \mathrm{~m}$ de hauteur bruni par les oxydes de fer (fig. 1 et 2). Dans la zone médiane (puits Viana) en effet et dans le secteur sud-est (puits Ramaix), le filon du Mur se confond en profondeur avec le filon du Centre (fig. 23), qui, dépourvu de chapeau de fer, ne commence qu'entre les niveaux 100 et $200 \quad\left(^{4}\right)$. Or l'exploitation antique n'ayant pas dépassé les niveaux 100/120, on conçoit que le filon du Centre n'ait pu être touché qu'occasionnellement et seulement dans sa partie supérieure (fig. 33). On peut donc considérer globalement que, de ce côté, c'est le filon du Mur qui a fait l'objet des travaux des Anciens.

A quelque $150 \mathrm{~m}$ à l'est, se trouve le filon du Toit, pourvu d'un chapeau de fer qui, au nord-ouest, a une puissance de 10

(2) Le gisement de Feitais, situé à $\mathrm{t} \mathrm{km}$ au nord-est des Algares, n'est pas encore en exploitation; il est lui aussi aveugle et n'a pas été repéré par les Anciens.

(3) Nous suivons ici Freire de Andrade, as minas de Aljustrel, p. 78 et Jazigo, p. 466. Filon du Toit, filon du Mur: ces dénominations proviennent $\mathrm{du}$ fait que, le gisement des Algares ayant été d'abord traité comme un seul gîte, la masse de l'est était considérée comme située au toit $\mathrm{du}$ gisement, celle de l'ouest au mur. «On appelle toit le plan droit ou ondulé qui forme la partie supérieure d'un gîte; le plan inférieur est le mur» (Burat, Traité du gisement, p. 290).

$\left.{ }^{4}\right)$ Niveau 100: $100 \mathrm{~m}$ au-dessous de la surface (pour le filon du Mur, la cote «zéro» est à la bouche du puits Yiana). Dans le secteur Sud-est, il faut ajouter au filon du Centre d'autres masses minéralisées, telles que le filon «Chalco» et le filon du Puits. 
à $15 \mathrm{~m}$. Mais, si Ton en juge par ce qui en reste (fig. 5 et 6), il était très difficilement repérable en surface. Il n'en a pas moins été intensément exploité dans Tantiquité. Nous reviendrons sur ce point à l'occasion du commentaire de Vip. i7( $\left.{ }^{5}\right)$, mais il est clair dès maintenant que le filon du Toit et le filon du Mur constituaient pour les Anciens les deux gisements principaux des Algares.

Effectivement, les vieux rapports - par exemple celui de 1873 - nous disent que les puits antiques étaient nombreux d'un bout à l'autre du filon Est et, avant les travaux modernes, se comptaient par plusieurs centaines sur le chapeau de fer du filon du Mur et à son voisinage $\left(^{6}\right)$.

\section{2. ${ }^{\circ}$ Gisement de S. João do Deserto}

Il n'avait qu'un affleurement, celui de la masse située au mur. On y comptait en 1873 neuf puits antiques ouverts au toit, donc à l'est du «crestón» ferrugineux. Tout ceci a disparu lors de l'ouverture de la «corta».

\section{$B$. Les travaux miniers antiques}

\section{1. ${ }^{\circ}$ Les sources}

Aucune étude spécifique des travaux antiques n'a été faite quand, au siècle dernier et au début du $\mathrm{XX}^{\mathrm{e}}$, les entreprises modernes ont attaqué les niveaux déjà exploités par les Anciens. Il faut le regretter, car aujourd'hui nous ne disposons sur cette ${ }^{*}$ il

$\left.{ }^{5}\right) \quad$ Vip. I désigne la table de bronze trouvée en 1876, Vip. Il celle qui fut découverte en 1906. Pour le commentaire de ces tables, voir la deuxième partie de notre étude.

$\left.{ }^{6}\right)$ Le même rapport (RIBEIRo et alii, Relatório, p. 6) signale encore de «vieux travaux» à l'ouest de l'affleurement du filon du Mur, principalement sur une étroite bande de schistes cuprifères, tachés de vert par les carbonates:

il s'agit sans doute de travaux superficiels - prospection ou très ancienne recherche d'oxydés — qui n'ont rien à voir avec l'intense exploitation par puits des filons de l'Est et du Mur. 
question que de maigres données: des observations générales

— existence de «vieux travaux», de puits, de galeries antiques

- quelques découvertes ponctuelles dues au hasard des travaux modernes - par exemple le puits à quatre treuils du niveau $\left.120{ }^{7}\right)$, le puits et la galerie coupés par un tunnel de roulage $\left(^{8}\right)$ - et surtout conservés dans les archives de la mine, les plans des travaux d'exploitation qui se sont déroulés entre 1903 et 1916.

Ces travaux sont présentés pilier par pilier et tranche par tranche $(9)$. Chaque tranche d'exploitation a fait l'objet d'un relevé en couleurs à l'échelle $1 / 200 \mathrm{e}$, où sont portés d'une part le tracé des minéralisations et les teneurs de ces dernières, d'autre part les travaux antiques rencontrés: puits, galeries, excavations, remblais. Ces relevés $\left({ }^{10}\right)$ concernent principalement le filon du

$\left(^{7}\right)$ VIANA et alii, Minerações romanas, p. 80-82. Nous reprenons infra la description de ces vestiges; voir aussi infra la figure 33 .

${ }^{8}$ ) Domergue-Freire de Andrade, Sondages à Aljustrel, p. 106. Cf. infra les figures 11 et 12 .

$\left.{ }^{9}\right)$ Dans une mine moderne, l'exploitation se déroule généralement de la façon suivante: le gisement est découpé transversalement en piliers verticaux et l'exploitation progresse par niveaux (à Aljustrel, de $20 \mathrm{~m}$ en $20 \mathrm{~m}$ ) ; à chaque niveau, les piliers sont abattus par tranches horizontales, le dépilage commençant par le bas et les vides laissés par l'abattage du minerai étant remblayés aussitôt. Pour plus de détails sur la méthode suivie par la «Société Anonyme Belge des Mines d'Aljustrel» à qui sont dûs les relevés en question, voir F INI L L s-L ou is, Les Mines romaines d'Aljustrel, p. 528-529.

$\left.{ }^{10}\right)$ Ils constituent six épais dossiers, dont voici le contenu:

dossier 1: fdon du Mur, niveau40; années 1904-1906.

dossier 2: filon du Mur, niveau60; année 1903.

dossier 3: filon du Toit, niveau60; années 1907-1909.

filon du Mur (filon «Chalco» et filon du Puits); années 1907-1908 .

dossier 4: filon du Mur, niveau80; année 1903.

dossier 5: filon du Toit, niveau80; années 1913-1915.

dossier 6: filon du Ravin, niveau 100; années 1914-1916.

São João, niveau 30; années 1914-1915.

L'existence de ces relevés nous avait été révélée par R. Freire de Andrade que nous remercions chaleureusement. Ce dernier en avait utilisé quelques-uns dans son article A lavra romana, p. 281-282, malheureusement amputé des illustrations correspondantes, ce qui ôte aux commentaires une grande part de leur intérêt. Pour notre part nous avons pu les consulter en mars 1980, grâce à l'amabilité de l'administration de «Pirites Alentejanas, S.A.R.L.», à laquelle nous adressons nos plus vifs remerciements. 
Mur - zone médiane (niveaux 40, 60, 80) et secteur sud-est (filon du Puits et filon "Chalco», niveau 60) (u) - et le filon du Toit (niveaux 60 et 80 ), ainsi qu'épisodiquement le gîte de S. João (niveau 30); on y trouve fréquemment mentionnés des travaux «romains», un terme que l'on doit toujours manier avec prudence mais qui, ici, vu l'importance et la durée de l'exploitation romaine dont témoignent en particulier les crassiers de scories visibles en surface, et vu les comparaisons que nous ferons plus loin avec les dispositions $\mathrm{du}$ règlement minier de Vipasca, nous paraît totalement justifié.

Ces relevés ne permettent malheureusement pas de restituer, à un ou plusieurs niveaux, l'intégralité des travaux romains sur toute la longueur des filons ni même sur un tronçon limité de 200 ou $300 \mathrm{~m}$ de longueur. Il n'est donc pas possible d'avoir une vue suffisamment vaste de leur organisation. Cette remarque vaut d'ailleurs aussi pour le plan Burthe-Gérard-Volpelière, publié par E. Cuq en 1911 (12) (fig. 26 et 27).

(n) On trouve aussi (dossier 6) des relevés du filon du Ravin (niveau 100) que nous ne savons où situer: vraisemblablement dans le secteur sud-est du filon du Mur.

(12) CuQ, Le développement de Vindustrie minière, p. 352-353 et 354. Nous avons reproduit ce plan à la figure 26. Y sont situés les 39 puits et les travaux antiques rencontrés jusqu'en 1911 aux niveaux 40 et 60 dans le filon du Mur et les filons annexes. Pour ce travail ont sans doute été utilisés les relevés mentionnés ci-dessus et d'autres encore, aujourd'hui perdus. Cependant il n'est rien moins que certain que sur eux toutes les structures antiques aient été indiquées. Ces relevés étaient en effet exécutés pour les besoins de l'exploitation moderne et non pour dresser le plan des travaux antiques, que l'on n'a pas recherchés spécialement. Il y a donc sûrement des lacunes, ce dont il faut tenir compte. Cuq ne l'a pas toujours fait; ainsi il n'est pas évident que, contrairement à son avis, ibid., p. 345, les puits $\mathrm{n}^{\circ} 11$ de notre figure 26, situés à l'écart et au mur du filon, soient à mettre en relation avec la longue bande de chantiers ouverts au toit. N'y avait-il pas d'autres puits d'accès, plus proches et qui auraient échappé à la curiosité des mineurs? En outre se pose le problème de l'interprétation et de la transcription des relevés: il est possible que celui que nous reproduisons à notre figure 28 (34e pilier, lère tranche) corresponde à une partie du plan Burthe-Gérard-Volpelière, à savoir les trois puits du $n^{\circ} 1$ et le puits $n^{\circ} 2$ situés au mur du filon du Mur (fig. 27): dans les deux cas il s'agit du niveau 40; la situation des puits, les espaces qui les séparent sont à peu 
Aussi pour notre part avons-nous préféré utiliser ponctuellement ces documents, en choisissant les relevés où la densité des structures antiques signalées paraissait garantir qu'on n'en avait pas oublié.

\section{2. ${ }^{\circ}$ S. João do Deserto}

Nous n'avons que peu de renseignements sur les travaux profonds de S. João. Nous savons seulement qu'il y en avait et que les galeries modernes les ont rencontrés: ainsi les deux puits

près semblables; en outre une précision de Cuq paraît le confirmer: «les (trois) puits inférieurs $(=\mathrm{au}$ mur)... sont dans un endroit où le terrain est si mauvais que les ingénieurs modernes ont préféré le contourner par une galerie circulaire», écrit-il; or une galerie de ce genre est bien indiquée sur le relevé. L'absence du puits et du travers-banc du niveau 40 signalés au toit (fig. 27) peut s'expliquer par l'état d'avancement des travaux, le relevé datant de 1905-1906 et le plan d'ensemble de 1911. Mais, sur celui-ci, tous les puits sont circulaires, alors que sur celui-là les deux plus au nord le sont, les autres étant rectangulaires; et surtout, sur le relevé de 1905-1906, le deuxième puits circulaire est désigné comme «soufflard» (ou puits d'aérage) et entouré d'un massif de protection, ce qui montre son utilisation à l'époque des travaux modernes, alors que sur le plan Burthe-Gérard-Volpelière, le deuxième puits est considéré comme ancien. Sans doute, objectera-t-on, il a un diamètre réduit $(1 \mathrm{~m})$, comme le premier: pourquoi donc ne s'agirait-il pas d'un puits antique réutilisé comme «soufflard» à l'époque moderne? Oui, mais, alors que chacun des trois autres puits est désigné nommément comme «puits romain», celui-là porte la seule mention de «soufflard». Enfin, au cas où l'on refuserait ce rapprochement, à quel autre emplacement du plan Burthe-Gérard-Volpelière pourraient être situés les travaux romains portés sur le relevé de 1906-1907? Si l'on connaissait la situation exacte du $34 \mathrm{e}$ pilier, il serait facile de s'en rendre compte; tel n'est pas le cas mais, de toute façon, on ne voit, sur le plan de la figure 26, aucun autre ensemble qui, au niveau 40 , corresponde au relevé du $34 \mathrm{e}$ pilier-lère tranche, que le deuxième des quatre puits soit maintenu ou non.

Ainsi donc on constate, à propos de cet exemple précis, que le plan Burthe-Gérard-Volpelière est soit inexact, soit incomplet. On ne peut donc s'appuyer sur lui pour étudier l'organisation de l'exploitation romaine. Et, $\mathrm{vu}$ ce que nous avons dit plus haut de la nature des relevés miniers, il en va de même pour tout plan d'ensemble qui s'appuierait sur eux et dont on voudrait tirer des conclusions sur l'organisation globale d'une mine romaine. 
qui sont signalés au toit du gisement, au niveau 40; de section carrée (1 $\mathrm{m}$ de côté), ils étaient situés à $4 \mathrm{~m}$ l'un de l'autre (13).

\section{$3^{\circ}$ Algares}

Nos informations sont plus importantes pour les Algares. La profondeur maximum atteinte est de $120 \mathrm{~m}$; la hauteur moyenne de la zone oxydée n'est que de $34 \mathrm{~m}$ environ. Mais cela ne signifie pas qu'au-dessous du chapeau de fer, les Anciens aient exploité la pyrite elle-même; simplement les variations du niveau hydrostatique ont fait que localement les zones d'oxydation et de cémentation descendaient plus bas. Selon Finiels et Louis, à qui nous devons de nombreuses précisions, les vieux travaux étaient particulièrement développés au toit du gisement $\left({ }^{14}\right)$, où la minéralisation était plus riche qu'au mur.

\section{a) Les puits}

Les Anciens avaient foncé un très grand nombre de puits, les uns en partant de la surface, les autres de l'intérieur entre deux niveaux. Il y en avait sur la crête même du chapeau de fer (fig. 8), mais surtout, vu le pendage, à l'est de cette dernière au toit. Toujours étroits, ils ont une section circulaire ( $O=0,90 \mathrm{~m}$ à $1 \mathrm{~m})$ ou carrée $(0,90 \mathrm{~m} / 1 \mathrm{~m}$ de côté $)$, parfois rectangulaire $(0,70 / 0,80 \mathrm{mxl} \mathrm{m}$ : fig. 28). Les puits de section plus importante (1,40 mxl,40 m) sont rares.

On retrouve encore de ces puits antiques au hasard des travaux modernes: ainsi, celui que nous avons vu en 1969 (fig. 11), de section rectangulaire $(1,20 \times 0,90 \mathrm{~m})$, muni sur une des grandes parois d'encoches espacées de $0,30 \mathrm{~m}$ à $0,40 \mathrm{~m}$ : il s'agissait donc d'un puits d'accès dans lequel les mineurs devaient monter et descendre soit en s'appuyant contre la paroi opposée, soit en s'aidant d'une corde pendant depuis le haut. Finiels (15) remarque que

(13) Arch. Mines Aljustrel, dossier n. ${ }^{\circ}$ 6: niveau 30, 5e pilier, 2e tranche.

(14) Finiels-Louis, Les mines romaines cTAljustrel, p. 529.

(15) Ibid., p. 530. 
certains puits avaient des cadres de soutènement en chêne vert constitués chacun de quatre éléments standards, donc interchangeables, longs de $1,30 \mathrm{~m}$, larges de $0,10 \mathrm{~m}$ à $0,12 \mathrm{~m}$ et épais de $0,06 \mathrm{~m}$. Les entailles des assemblages étaient à mi-bois, pratiquées sur la hauteur de la planche de chêne; les planches étaient placées de chant pour former les cadres qui étaient posés jointifs. Dans chaque cadre, les éléments femelles se faisaient face, mais les cadres alternaient, les éléments mâles étant placés 1 et 3 dans l'un, 2 et 4 dans le suivant. $\mathrm{Vu}$ la solidité de ces étayages, Finiels pense que les puits ainsi revêtus étaient des puits d'extraction.

Cette opinion est confirmée par la découverte d'un puits intérieur ( $\left.{ }^{16}\right)$ muni de quatre treuils d'extraction, faite en 1921 et publiée en 1954 par Viana, Freire de Andrade et Veiga Ferreira $\left({ }^{17}\right)$. La section du puits (fig. 33) est approximativement de $1 \mathrm{mxl} \mathrm{m}$ et, sur le dessin, seule l'alternance des cadres n'est pas indiquée. Chacun des treuils était constitué par un tambour tournant sur les fourches de deux supports verticaux et mu par deux cabestans verticaux de quatre bras, un à chaque extrémité; la partie centrale du tambour était de section carrée pour que le câble pût s'enrouler sans glisser; auparavant, le câble passait dans la gorge d'une poulie de gros diamètre dont l'axe reposait sur un support analogue au premier et placé en avant de lui; en outre un rouleau fixe sur la paroi du puits, près du bord, permettait au câble de glisser sans s'user sur l'arête tranchante du dernier cadre. Le fond de ce puits était à la cote 120 ; les treuils se trouvaient $8 \mathrm{~m}$ plus haut, au niveau 112. Les câbles et les couffins en spart utilisés pour l'extraction furent trouvés sur place; ils sont semblables à ceux

(16) On appelle «puits intérieur» un puits qui ne débouche pas au jour. Les puits intérieurs sont utilisés pour la poursuite de l'exploitation en profondeur.

(17) Vian a et alii, Minerações romanas, p. 80-82. Il ne saurait s'agir, malgré les apparences, du puits ou d'un des puits étudiés par Finiels et Louis, Les Mines romaines d'Aljustrel, p. 529-530; en effet les Archives de la Mine conservent de P. Finiels un manuscrit dactylographié daté de 1903, qui renferme déjà les observations techniques contenues dans l'article publié en 1936 en collaboration avec Louis: il s'agit donc de deux vestiges différents, chacun corroborant la réalité de l'autre. 
qui sont conservés à Lisbonne aux Services Géologiques; en revanche les poulies du Musée de Belém (fig. 14) sont d'un diamètre nettement inférieur à celui des treuils décrits ci-dessus.

Dans de nombreuses mines du Sud-ouest de la Péninsule (province de Huelva, en Espagne, et Sud du Portugal) où les gîtes sont du même type que celui d'Aljustrel et où l'exploitation romaine a été très importante, on a remarqué que les puits étaient fréquemment groupés deux par deux, d'où l'expression de «puits jumeaux» souvent employée à leur égard $\left({ }^{18}\right)$. Les ensembles les plus remarquables se trouvent à La Zarza (H 19), Sotiel Coronada (H 20), Cabezas del Pasto (H 39) $\left({ }^{19}\right)$. D'après un exemple que nous avons étudié sur ce dernier site, nous avons pensé que l'un des deux puits, muni d'encoches superposées sur une seule paroi ou sur deux parois se faisant face, était le puits de descente pour le personnel, l'autre aux parois lisses le puits d'extraction du minerai. Des puits jumeaux paraissent être indiqués sur le plan de la figure $26\left({ }^{20}\right)$ et il est possible que le puits de la figure 11 ait appartenu à un couple de ce genre et par ailleurs nous rappelons les deux puits de $\mathrm{S}$. João, déjà signalés, qui, éloignés seulement de $4 \mathrm{~m}$ l'un de l'autre, ont pu en constituer un.

A Sotiel Coronada et à La Zarza plus particulièrement (fig. 35 et 36), la distribution régulière de ces couples de puits fait penser à une division des gisements en concession d'égales dimensions. En était-il de même à Aljustrel ? Nous reviendrons là-dessus dans notre commentaire de Vip 77, mais dès maintenant l'examen de quelques-uns des plans de travaux modernes (fig. 28 à 32) nous conduit à faire à ce propos quelques remarques intéressantes.

(18) Voir là-dessus, dans Domergue, Mines, le chapitre concernant les techniques d'exploitation. A remarquer aussi le témoignage de Deligny, Notice, p. 901: «Lorsque les puits devaient avoir une grande profondeur, on faisait des puits jumeaux, afin d'assurer la ventilation. Ces puits, qui n'avaient pas plus de $0,90 \mathrm{~m}$ à $1 \mathrm{~m}$ sur $0,70 \mathrm{~m}$ à $0,80 \mathrm{~m}$, allaient ainsi à plus de $80 \mathrm{~m}$ de profondeur».

(19) Les indications du type $\mathrm{H}$ 19, H 20, etc. renvoient à notre Catalogue des mines et fonderies antiques de la Péninsule Ibérique, province de Huelva.

$\left(2^{\circ}\right)$ pig. $26, n^{\circ} 6,8,11$. Dans le cas du $n^{\circ} 1$ (au toit), le puits relevé au niveau 60 est sûrement un puits intérieur. 
Ainsi nous sommes frappé par la régularité de l'espacement des structures antiques telles que puits et travers-bancs. Sur la figure 28 une distance de $8,50 \mathrm{~m}$ sépare les puits 1 et 2 (21) et on la retrouve normalement à quelques mètres au-dessous entre les mêmes puits (fig. 29) (22); par ailleurs les puits 2 et 3 de la figure 28 sont séparés par $24 \mathrm{~m}$, soit 3 fois $8 \mathrm{~m}$. A la figure 30 , on mesure $9,50 \mathrm{~m}$ entre les puits 1 et 2 et $16 \mathrm{~m}$ (soit deux fois $8 \mathrm{~m}$ ) entre les puits 2 et 4 , tandis que le travers-banc 3, qui se dirige vers une veine minéralisée située plus à l'ouest, est à égale distance de ces deux derniers puits. Sur la figure 32, les deux travers-bancs sont distants de $15 \mathrm{~m}$ l'un de l'autre. Un intervalle constant qui varie de $7,50 \mathrm{~m}$ à $9,50 \mathrm{~m}$ - mais il atteint le plus souvent $8 \mathrm{~m}$ — semble donc d'après ces exemples séparer les diverses structures d'accès aux chantiers. Si ces structures appartiennent respectivement à des concessions constituées par des sections verticales du gisement découpé régulièrement, cet intervalle pourrait être aussi la mesure de la largeur des concessions. Elle équivaudrait à un nombre rond de 30 pieds romains $\left({ }^{23}\right)$.

\section{b) L'abattage et les chantiers}

Le petit nombre de pics en fer recueillis et conservés s'explique sans doute par le rôle que joue tout fragment de fer dans le processus chimique de cémentation $\left({ }^{24}\right)$ : nous n'en connaissons que deux. En tout cas pics et coins étaient les outils utilisés pour l'abattage; selon Finiels $\left({ }^{25}\right)$, celui-ci était organisé rationnellement et, à l'inté-

(21) Les distances sont mesurées d'axe en axe.

(22) Des remblais romains importants sont signalés sur le relevé de la 7e tranche (niveau 60) de ce même pilier: ils emplissaient des chantiers qui doivent être mis en relation avec ces puits.

(23) Le pied romain vaut $0,2944 \mathrm{~m}$.

(24) Finiels-Louis, Les mines romaines (TAljustrel, p. 532. Ce procédé a été communément utilisé au XIXème et au XXème siècle dans les mines de pyrite du Sud-Ouest de la Péninsule pour récupérer le cuivre contenu par les eaux acides issues des travaux profonds: il consistait à mettre des eaux en contact avec des déchets de ferraille pour précipiter le cuivre.

(25) Finiels-Louis, Ibid., p. 533-535. 
rieur d'un chantier, niveau par niveau, section par section et tranche par tranche, de bas en haut. On pourrait craindre qu'ici la vision de l'ingénieur n'ait été faussée par l'habitude des techniques modernes; or le dessin d'un chantier découvert en 1920-1921 vient encore corroborer les dires de Finiels (fig. 33): on y voit un échafaudage à trois niveaux, constitué par des troncs d'arbre munis d'encoches appuyés obliquement contre les parois d'un filon et supportant des madriers horizontaux; le niveau supérieur est le front de taille. En bas, s'accumulent les stériles résultant d'un premier tri et destinés à remblayer le vide provoqué par l'abattage de la section. Le piétinement des mineurs, l'action des eaux et de la chaleur finissaient par transformer ces remblais en masse assez compacte pour éviter les effondrements lors de l'abattage du pilier suivant; ces remblais furent exploités au siècle dernier, car, à $60 \mathrm{~m}$ de profondeur, ils contenaient en moyenne $1 \%$ de cuivre et à $40 \mathrm{~m}$ encore près de $0,50 \%$; plus haut il n'y avait plus $\mathrm{du}$ tout de cuivre. C'est dans ces remblais que furent retrouvés la plupart des outils et ustensiles antiques. La découverte d'une meta en pierre dure (diam. : 0,45 m) suggère qu'on a pu procéder, dès le premier tri, au broyage du minerai.

Lorsque les épontes étaient solides, les chantiers pouvaient ne pas être remblayés. On remarque par exemple dans le filon du Mur, au niveau 60, des vides dont le volume dépasse $70 \mathrm{~m}^{3}$ et qui sont maintenus par des piliers de soutènement renfermant des minerais à $4,87 \%$ de cuivre $\left({ }^{26}\right)$.

(26) Filon du Mur, 33e pilier, 5e tranche: 2 vides dont l'un de $50 \mathrm{~m}^{3}$ et l'autre plus vaste encore; $29 \mathrm{e}$ pilier, $4 \mathrm{e}$ tranche: 1 vide de $70 \mathrm{~m}^{3}$ (L.: $\left.8,60 \mathrm{~m}\right)$. Des remblais importants sont à noter, toujours au niveau 60 du filon du Mur, dans le 30e pilier, 8e tranche (L.: $12 \mathrm{~m}$ ); L: $5 \mathrm{~m}$ ) et dans le 34e pilier, 7e tranche. D'ailleurs, dans ce secteur du filon du Mur, du 29e au 34e pilier et entre la $3 \mathrm{e}$ et la $8 \mathrm{e}$ tranche, c'est une succession presque ininterrompue de vides et de remblais antiques (Arch. Mines cTAljustrel, Plans d'exploitation, 2e dossier). 


\section{c) Les galeries}

La circulation entre les chantiers et les puits se faisait par le moyen de galeries voûtées, de petite section (h.: 0,90 m;

1. : $0,80 \mathrm{~m}$ ) (fîg. 12), munies de niches pour les lampes ou encore par des descenderles $\left({ }^{27}\right)$. Quand ces galeries traversaient des terrains sans consistance, on avait recours au boisage. Des troncs de chênes verts, creusés d'encoches, servaient d'échelles dans les passages difficiles (fîg. 13).

\section{d) La régularité des travaux}

Toutes ces observations suggèrent que, dans les parties du gisement exploitées par les Anciens, les travaux se sont déroulés d'une façon méthodique $\left({ }^{28}\right)$; on ne se trompera sans doute pas en attribuant ces travaux à l'exploitation rationnelle d'époque romaine que suggèrent les dispositions de Vip. 7/(29). Car antérieurement, et surtout à l'Enéolithique et à l'Age du Bronze, où, comme nous le verrons plus bas, le gisement a été exploité, les travaux ont dû être moins rationnels.

\section{e) Uexhaure}

Un problème est celui de l'exhaure. Les dispositions de Vip. 11 14 à 18 plaident fortement en faveur de l'existence d'un travers-banc romain destiné à évacuer les eaux de mine. Mais aucun rapport

(27) L'une d'elles est signalée dans le filon du Toit, 33e pilier, 7e tranche (Arch. Mines d'Aljustrel-Plans d'exploitation, 5e dossier).

(28) Contra, Freire de Andrade, A lavra romana, p. 280. Le fait qu'on ne peut avoir des travaux antiques une vision globale et l'aspect actuel des chantiers qui, au cours de quelque dix-huit siècles, ont eu largement le temps de se détériorer expliquent pour une grande part une telle opinion, Par ailleurs, il est bien vrai que souvent, en particulier dans des gîtes filoniens, les travaux sont irréguliers et désordonnés. Mais, dans les mines du Sud-ouest, vu l'allure des gisements et surtout l'organisation imposée par le fisc, il semble bien qu'ils ont été plus rationnels et davantage ordonnés.

${ }^{29}$ ) Voir le commentaire que nous en donnons dans la deuxième partie de cette étude. 
ni article du siècle dernier n'en parlent, bien qu'à cette époque le premier souci des sociétés minières qui entreprenaient l'exploitation des gîtes métalliques, en particulier dans le Sud-ouest de la Péninsule, fût de retrouver le travers-banc d'exhaure antique et de le mettre en état au plus tôt, afin de dénoyer les niveaux supérieurs de la mine, de les explorer et éventuellement de les exploiter dans les meilleurs délais. C'est dans ces conditions qu'ont été découverts les travers-bancs romains des mines du Sud-ouest suivantes: Concepción (H 6: «socavón Carmen»), filon Nord à Tharsis (H 12: «La Amargasilla»), La Zarza (H 19: «Los Cepos» et «La Algaida»), Sotiel Coronada ( $\mathrm{H}$ 20) dans la province de Huelva; S. Domingos (POR 7) et Serra de Caveira (POR 36), les deux autres grands gîtes pyriteux du Portugal méridional.

Aux Algares, vu la topographie du site et le pendage du gisement, un tel travers-banc devait atteindre ce dernier à l'est et déboucher près de la «ribeiia dos Feitais». Or il y en a effectivement un et son entrée est toujours visible (fig. 7), mais il est connu sous le nom de «galeria da Transtagana» ${ }^{30}$ ), ce qui semblerait signifier qu'il a été creusé par la Companhia de Mineração Transtagana, à laquelle la mine avait été concédée de 1867 à 1881 . Le rapport de 1876 parle des travaux qui furent réalisés cette année-là pour l'élargissement et l'augmentation de pente de la «galeria geral d'esgoto» ${ }^{(31)}$. Cette dernière existait donc déjà auparavant mais elle était étroite et sa pente insuffisante. Il est peu probable que, dans ce premier état, elle ait été l'oeuvre de la Transtagana; celle-ci n'a en effet la concession d'Aljustrel que depuis $1867\left({ }^{32}\right)$, et si, après cette date, elle avait entrepris

$\left(3^{\circ}\right)$ Freire de Andrade, A lavra romana, p. 278.

(31) Companhia de Mineração Transtagana- Gerencia de 1876 (dactylographié), p. 7. Le paragraphe concerné porte le titre suivant: "Alargamento e rectificação da galeria geral d'esgoto» et nous en citons les passages les plus significatifs: "Subordinada en grande parte a extracção á galería geral d'esgoto, era urgente colocá-la en circonstancias de bem servir. Estava projectado o alargamento e rebaixo do pizo para dar ás águas maior corrente... Esta grande reparação, que foi feita nos 12 mezes, na extensão de 800 m, em rocha metamorphica bastante dura...».

(32) Voici en quelques dates l'histoire des mines d'Aljustrel à l'époque 
un tel travail, elle aurait sans doute tracé le travers-banc de façon à ne pas avoir à l'élargir et à rectifier sa pente quelques années plus tard.

Nous ne savons pas grand chose de l'activité de la «Lusitanian Mining $\mathrm{G}^{0}$ », qui a précédé la Transtagana; elle fut sans doute la première à l'époque moderne à exploiter les Algares, où une galerie recoupant le gisement au toit lui est attribuée par le rapport de $1876{ }^{(33)}$. Mais a-t-elle aussi percé le travers-banc d'exhaure? Si tel avait été le cas, il eût été visible et par conséquent porté sur le plus ancien plan de surface des Algares que nous possédions, celui de 1867 (fig. 24) ( $\left.{ }^{34}\right)$. Or il n'en est rien, ou plutôt il y a

moderne (d'après Allan, A mineração em Portugal, p. 11 et Freire de ANDRADE, As minas de Aljustrel, p. 75-77).

- 1847: première concession, sans doute à D. Sebastião de Gargamala et seulement pour le gîte de S. João.

- 22 avril 1854: fin de la concession.

- septembre 1854: visite et rapports de Carlos Ribeiro et João Ferreira Braga (reproduits dans Freire de Andrade, Documentos inéditos, p. 337-351): ces rapports signalent l'intérêt du gisement des Algares, tandis que jusqu'alors, semble-t-il, seul celui de S. João avait été exploité.

- 1854 ou peu après: concession à la «Lusitanian Mining $\mathrm{C}^{\circ}$ » (d'après Allan, «Lusitanian Mines, Ltd»). Exploitation du gîte des Algares, dans la zone des Moinhos Caídos (Allan), ou plutôt du Moinho Caiado, situé sur le chapeau de fer des Algares, et qui donnera son nom au futur puits Caiado.

- 27 avril 1866: fin de la concession.

— 1867: concession à la «Companhia de Mineração Transtagana».

- 1868: début de l'exploitation du gisement par la Transtagana.

- 1881: fin de la concession d'Aljustrel à la Transtagana.

- 1895: concession à la banque Fonsecas, Santos et Viana.

— 1898: concession à la «Société Anonyme Belge des Mines d'Aljustrel».

— 1975: concession à la compagnie «Pirites Alentejanas S.A.R.L.».

(33) Est-ce celui qui est indiqué à l'extrémité sud-est du chapeau de fer des Algares, sur la figure 24? C'est possible.

(34) Original daté du 30 septembre 1867. Fac-similé dans Freire de Andrade, Documentos inéditos, d'après une copie («copia fiel») exécutée le 15 janvier 1901. Notre propre figure 24 est un calque de cette dernière. $\mathrm{Vu}$ sa date, l'original est sans doute le plan de surface qui a été exécuté au moment où la Transtagana a obtenu la concession. $\mathrm{Y}$ sont portés tous les travaux visibles en surface.

Plusieurs plans anciens sont conservés dans les archives de la mine. Outre la copie de celui de 1867, nous avons plus particulièrement consulté:

IB: sans date $\left(=\mathrm{IB}^{1}\right)$. Ech.: 1/2500e. Papier cansón beige, recollé 
bien, à l'est du moulin Caiado, une série de trois puits alignés, unis par un double tireté et qui, par leur situation, paraissent correspondre aux puits de jour du travers-banc les plus proches du chapeau de fer, tels qu'ils sont représentés sur le plan plus tardif de la figure 25. Mais la lettre $A$, mentionnée d'abord auprès des puits, ensuite à l'endroit où s'interrompt le double tireté paraissant représenter un tronçon de galerie long de $130 \mathrm{~m}$ (fig. 24), a bien des chances de signifier «antigo». Cela indiquerait qu'en 1867 on avait repéré au toit $\mathrm{du}$ filon $\mathrm{du}$ mur une galerie munie de trois puits de jour, que cette galerie était considérée comme antique (A) $h t$ qu'elle n'avait pas encore été explorée sur toute sa longueur.

Il apparaît donc que, selon toute probabilité, la «galeria geral d'esgoto» n'est l'oeuvre ni de la «Companhia de Mineração Transtagana», ni de la «Lusitanian Mining $\mathrm{C}^{\circ}$ ». En revanche, on a de bonnes raisons de penser qu'il doit s'agir d'un travers-banc antique dont seul un tronçon était connu en 1867, lorsque la Transtagana obtint la concession de la mine. Connaissant l'intérêt que témoignaient alors les compagnies minières à la découverte et à la remise en état de tels ouvrages, on peut s'attendre que la Transtagana n'eut pas de tâche plus urgente que d'en terminer le dégagement pour enfin disposer d'une «galeria d'esgoto». Ce qui fut fait dès $1873\left({ }^{35}\right)$. Dès lors on ne s'étonnera pas que, sur des plans plus tardifs $\left({ }^{36}\right)$, le travers-banc soit facilement

avec de la toile aux endroits déchirés. Manque le coin supérieur droit, où devait être mentionnée la date. Très mauvais état.

IB: sans date $\left(=\mathrm{IB}^{2}\right)$. Ech. : 1/5000e. Papier cansón beige, entièrement recollé sur toile. Mauvais état. Nous en avons tiré le calque d'après lequel est faite la figure 25 et duquel est exclus le tracé des installations d' «ustullaçâo» et des limites de propriétés.

2B: (primitivement 3 B), 25 août 1899. Ech.: 1/5000e. Papier toile blanc.

Les deux premiers plans portant la même cote, nous les avons différenciés: $\mathrm{IB}^{1}$ et $\mathrm{IB}^{2}$. $\mathrm{Vu}$ l'ordre adopté par les archives de la mine pour le classement de ces plans, il est probable que les plans $\mathrm{IB}^{1}$ et $\mathrm{IB}^{2}$ sont antérieurs à $2 \mathrm{~B}$.

${ }^{(35)}$ Le rapport de R IB EIRO et alii, Relatório, p. 9, le mentionne.

(36) Plans $1 \mathrm{~B}^{1}$ et $1 \mathrm{~B}^{2}$ de la note 34 . En revanche rien n'est signalé sur le plan $2 \mathrm{~B}$. 
repérable sur toute sa longueur, avec ses puits de jour et son entrée ( ${ }^{37}$ ) (fig. 26). Il coupait le gisement des Algares à $60 \mathrm{~m}$ environ au-dessous de l'affleurement du filon du Mur.

La façon dont il a été tracé et creusé nous paraît confirmer notre opinion. On retrouve là en effet la marque de la technique romaine: creusement par tronçons à partir de puits de jour( $\left.{ }^{38}\right)$, tracé établi en fonction des talwegs existant en surface de façon à réduire au maximum la profondeur des puits de jour et le travail de levage des déblais produits par le traçage des divers tronçons. Le travers-banc de la Algaida à la Zarza (H 19) et l'un de ceux de Riotinto (H 40) constituent d'autres exemples de ce type d'ouvrage ( $\left.{ }^{39}\right)$.

Ainsi donc il apparaît que la «galeria da Transtagana» ne peut être autre chose que le cuniculus qui aquam metallis subducet de Vip. $/ /(40)$. Son tracé, long de quelque huit cents mètres,

(37) Elle a été ajoutée au crayon sur le plan $1 \mathrm{~B}^{2}$.

(38) Il est vrai que cette méthode est encore recommandée au XIXème siècle pour le percement des galeries d'écoulement ou de tout ouvrage comparable (cf. Bu RAT, Traité $d u$ gisement, p. 144). Mais ici tous les autres indices - le tracé, la comparaison avec les autres mines antiques principalement celles du Sud-ouest, l'exégèse des plans du XIXème siècle — s'accordent pour qu'on fasse du travers-banc d'Aljustrel un ouvrage romain.

(39) Pour plus de détails sur ce point, voir, dans Domergue, Mines, le chapitre concernant les techniques.

${ }^{40}$ C'était déjà l'opinion de Freire DE ANDRADE, A lavra romana, p. 279. Encore fallait-il le démontrer. Peut-être pensera-t-on que nous nous sommes trop longuement attardé sur ce problème. Mais il nous a paru indispensable de le résoudre, car, pour comprendre les paragraphes 14 à 18 de Vip. II, il importe que l'existence réelle d'un travers-banc d'exhaure romain à la mine des Algares soit bien établie.

Par ailleurs l'hypothèse d'un travail arabe est peu probable, et, en plus des preuves que nous avons fournies ci-dessus, il paraît plus logique d'attribuer l'exécution de ce travers-banc aux Romains qui ont laissé tant de traces de leur activité minière et métallurgique à Aljustrel plutôt qu'aux Arabes, dont les Algares n'ont guère conservé de témoignage de leurs travaux, bien que le nom du site, «El Algar», désigne la mine en arabe.

Après la Reconquête, le site est attribué par D. Sancho II à l'ordre de Santiago, comme le montre un document de 1235 [Arch. Nac., «Livro dos Mestrados», f. 170 v.) cité par Estácio da VeigA, A tabula de bronze, p. 38. Les ferraria mentionnés par ce document doivent être les crassiers de scories 
suivait le fond d'un talweg, affluent temporaire de la rive gauche de la ribeira dos Feitais; il était jalonné par six (ou huit) puits de jour (fig. 33 et 25) (41); il atteignait le gisement des Algares au toit à $60 \mathrm{~m}$ de profondeur environ.

Il reste qu'au-dessous de ce niveau, les travaux se sont poursuivis encore jusqu'à $100 / 120 \mathrm{~m}$ de profondeur. Il faut donc que de ces niveaux jusqu'à celui du travers-banc, l'épuisement des eaux ait été effectué par d'autres moyens. On n'a pas trouvé à Aljustrel de batteries de vis d'Archimède ou des séries de roues à augets comme dans d'autres mines de la Péninsule $\left({ }^{42}\right)$, ce qui ne signifie pas qu'il n'y en ait pas existé. En tout cas, localement, à l'intérieur de la mine, on a pu utiliser des seaux en bronze (fig. 16) que les treuils élevaient $\mathrm{du}$ fond des puits et dont on versait le contenu dans des caniveaux ou des rigoles en bois (fig. 13) qui conduisaient l'eau jusqu'au travers-banc. Des récipients en bois munis ou non d'un manche (fig. 16) semblent avoir servi à écoper.

dont la teneur en fer est, comme on sait, toujours très élevée. Quant au terme adicia employé dans ce même texte, il est absent du Glossarium mediae et infimae latinitatis, de Du Cange. En revanche, dans le Novo Dicionário da Lingua Portuguesa, de Cândido de Figueiredo, 4ème édition, Lisbonne, sans date, on trouve adiça, mot ancien désignant une «mina de oiro». Ainsi donc, au XlIIème siècle, on recherchait l'or à Aljustrel: sans doute l'or natif dans les niveaux supérieurs des gisements.

${ }^{41}$ Six puits seulement, régulièrement espacés, sont portés sur le plan $1 \mathrm{~B}^{*}{ }^{1}$. Sur le plan $1 \mathrm{~B}^{2}$ (fig. 25), deux puits plus resserrés sont indiqués entre le dernier (compté à partir de l'entrée du travers-banc) des six puits. Et il y a les 3 puits du plan de 1867 (fig. 24). Alors, six, huit, ou neuf puits?

(42) Vis d'Archimède: El Centenillo ( $\mathrm{J}$ 12), Sotiel Coronada (H 20). Roues à augets: Tharsis (H 12), Riotinto (H 40), S. Domingos (POR 7), etc. Voir là-dessus, dans Doмe RGUE, Mines, le chapitre sur les techniques minières romaines. 


\section{Les minerais exploités. Les métaux produits}

\section{$10 \quad$ Le cuive}

11 est clair que les minerais de cuivre oxydés (carbonates) et les riches sulfures soit primaires (chalcopyrite) soit secondaires (chalcosite) ont été exploités par les Anciens: plusieurs des remblais le prouvent; nous en avons recueilli des échantillons tant sur l'oppidum de Mangancha que dans l'habitat du chapeau de fer des Algares (43); enfin, lors d'un ultime triage avant la fusion, des minerais jugés trop pauvres ont été rejetés et on les trouve abandonnés dans les crassiers tels les petits blocs de quartz ou les fragments de schiste teintés de malachite que nous y avons souvent remarqués. On a aussi découvert dans les scories un lingot de cuivre.

(43) Analyse d'un échantillon trouvé dans l'habitat romain du chapeau de fer des Algares (1er siècle de notre ère): $\mathrm{Pb} 0,23 \% ; \mathrm{Zn} 0,80 \% ; \mathrm{Cu} 23,75 \%$ (Laboratoire des mines d'Aljustrel).

Analyses spectrographiques de minerais réalisées par J. Bourhis, ingénieur G.N.R.S. au Laboratoire $d^{\wedge}$ Anthropologie, Préhistoire, Protohistoire et Quartenaire Armoricains de Rennes (arc sur globule: technique de l'arc continu).

\begin{tabular}{|c|c|c|c|c|c|c|c|c|c|c|c|c|}
\hline$N^{\circ}$ & Nature & $\mathrm{Cu}$ & $\mathrm{Sn}$ & $\mathrm{Pb}$ & As & $\mathrm{Sb}$ & Ag & $\mathrm{Ni}$ & $\mathrm{Bi}$ & $\mathrm{Fe}$ & $\mathrm{Zn}$ & Mn \\
\hline 314 & malachite & +++ & 0,002 & 0,002 & 0,001 & - & - & 0,05 & - & 0,80 & 0,08 & 0,05 \\
\hline 315 & malachite & 65,5 & 0,002 & 0,001 & 0,005 & - & 0,001 & 0,06 & - & + & 1 & 0,01 \\
\hline 316 & malachite & 53,4 & $<0,001$ & 0,001 & 0,001 & - & - & $<0,001$ & - & 1,5 & 0,50 & 0,005 \\
\hline 317 & chalco. & +++ & 0,05 & 0,08 & 1 à 3 & 0,15 & 0,008 & 0,005 & 0,05 & + & 0,50 & 0,008 \\
\hline 318 & malachite & ++ & 0,002 & 0,005 & 0,01 & - & - & 0,01 & $<0,001$ & + & 0,10 & 0,02 \\
\hline 319 & azurite & 43,9 & 0,003 & 0,15 & 0,008 & $<0,001$ & - & - & 0,001 & $\sim 8$ & 0,01 & 0,01 \\
\hline 320 & malachite & 5,2 & - & 0,01 & 0,01 & $<0,001$ & $<0,001$ & 0,02 & - & 2,5 & 3 & 0,50 \\
\hline
\end{tabular}

Les numéros sont ceux de la série d'analyses qui constitue un appendice à notre Catologue. Teneur comprise entre 1 et $10 \%$ : + , supérieure à $50 \%:+++$, comprise entre 10 et $50 \%:++$.

Les échantillons proviennent de l'oppidum de Mangancha (couche du 1er s. av. J.-C.: nos 314 à 316), de l'habitat du chapeau de fer des Algares (couche du 1er s. ap. J.-C.: $n^{\circ}$ 317) (couche du lile s. ap. J.-C.: $n^{\text {os }} 318,319$ ). Le $n^{\circ} 320$ a été trouvé dans un crassier romain du vallon de Feitais. 
Selon R. Freire de Andrade (44), la teneur limite en cuivre des minerais exploités par les Romains est de 1 ordre de $2 \%$ dans les niveaux supérieurs (jusqu'au niveau 60), tandis que dans les niveaux inférieurs, du minerai à 15 ou $18 \%$ de cuivre n'avait pas été abattu. Par ailleurs, les remblais des niveaux supérieurs sont à peu près stériles, alors qu'au-dessous ils peuvent renfermer des fragments de minerai dont la teneur en cuivre est de l'ordre de 1 à $3 \%$.

Les quelques sondages que nous avons effectués dans les plans d'exploitation des niveaux 40 à 100 confirment à peu près ce point de vue, au moins pour les niveaux supérieurs. Il est néanmoins difficile d'en faire une loi générale, comme le montrent les quelques cas exposés ci-dessous:

1_ Niveau 40, filon du Mur (fig. 28) (45): quoique percée à son extrémité nord par le puits romain 2, une veine minéralisée longue de $15 \mathrm{~m}$ et puissante de $1 \mathrm{~m}$, dont la teneur en cuivre était supérieure à $1 \%$ et pouvait atteindre $14 \%, \mathrm{n}$ a pas été abattue. Il est vraisemblable que le souci de maintenir autour $\mathrm{du}$ puits un massif de protection a empêché la reconnaissance de la veine. La même situation se retrouve dans le même pilier au niveau 60 (fig. 29), où deux veines à $7 \%$ de cuivre encadrent le puits 2 et, proches du puits 1 , n'ont pas été touchées.

2 -Niveau 60, filon du Mur( $\left.{ }^{4 e}\right)$ : sur le côté d'un chantier dont on a parlé plus haut et qui avait laissé dans le filon un vide important maintenu par des piliers de minerai iiche, un massif minéralisé à $6 \%$ de cuivre n'a pas été enlevé, sans doute parce qu'il était entouré d'un minerai pauvre dont la teneur en cuivre était inférieure à $2 \%$.

3 _ Niveau 60, filon Est $\left({ }^{47}\right)$ : un travers-banc venant de l'ouest et tracé dans le stérile s'est arrêté à $2 \mathrm{~m}$ d'un massif minéralisé à $2 \%$ de cuivre; s'il avait pénétré dans la veine, il aurait trouvé à quelques mètres au sud, des minéralisations a 5 ou $6 \%$ de cuivre.

(44) Freire de Andrade, A lavra romana, p. 281.

(45) Arch. Mines d'Aljustrel, Plans d'exploitation: 34e pilier, 1ère tranche, niveau $40\left(\right.$ dossier $\left.n^{\circ} 1\right)$ et 8 e tranche, niveau $60\left(\operatorname{dossier} n^{\circ} 2\right)$.

$\left({ }^{4} \ll\right)$ Ibid., $29 \mathrm{e}$ pilier, $3 \mathrm{e}$ tranche $\left(\operatorname{dossier} \mathrm{n}^{\circ} 2\right)$.

$\left({ }^{47}\right) \quad$ Ibid., 31e pilier, $4 \mathrm{e}$ tranche $\left(\right.$ dossier $\left.\mathrm{n}^{\circ} 3\right)$. 
4 - Niveau 60, filon du Puits $\left({ }^{48}\right)$ : l'extrémité d'un traversbanc s'est élargie en un chantier assez vaste, mais où l'abattage a été arrêté bien que la veine abattue fût minéralisée avec des teneurs variant de 6,37 à $10 \%$ de cuivre.

b - Niveau 60, filon de l'Est (fig. 31) (49): un puits romain se trouve à $5 \mathrm{~m}$ à l'ouest du mur du gisement, alors qu'une veine minéralisée à $6 \%$ de cuivre court en avant de lui sur $30 \mathrm{~m}$ de longueur sans avoir été touchée. Le voisinage du travers-banc d'exhaure explique l'absence de recherche dans ce secteur comme le prescrivent les dispositions de Vip. II $\left({ }^{50}\right)$.

6 - Niveau 80, filon de l'Est $\left({ }^{51}\right)$ : la descenderle romaine déjà mentionnée plus haut longe l'extrémité d'une importante veine (longueur: $15 \mathrm{~m}$; largeur: $3 \mathrm{~m}$ ) minéralisée en cuivre avec des teneurs de l'ordre de 1 à $5 \%$. Ici effectivement la recherche de minerais plus riches à des niveaux plus profonds a pu faire négliger cette veine.

7 - Niveau 100, filon du Ravin (52): un puits et deux petits travers-bancs romains sont signalés au mur du gisement, à 2 ou $3 \mathrm{~m}$ d'une veine dont le minerai, sur $15 \mathrm{~m}$ de longueur et $5 \mathrm{~m}$ de puissance, a une teneur en cuivre proche de $18 \%$.

Les cas qui viennent d'être évoqués appellent les remarques suivantes:

- il est difficile de dire que les Romains ont négligé des amas de minerai riche en cuivre quand ni leurs puits, ni leurs travers-bancs ne les ont touchés (cas nos 3 et 7). En revanche cela montre la difficulté qu'ils avaient à reconnaître les gisements. Le cas $n^{\circ} 5$ résulte de l'application du règlement minier.

- lorsque de toute évidence des minéralisations suffisamment riches pour intéresser les mineurs ont été touchées par leurs travaux sans que l'exploitation ait été poursuivie, il peut se faire que des circonstances spéciales justifient cet apparent dédain: maintien

(48) Ibid., 7e pilier, 1ère tranche $\left(\right.$ dossier $\left.\mathrm{n}^{\circ} 3\right)$

(49) Ibid., 27e pilier, 1ère tranche $\left(\operatorname{dossier} \mathrm{n}^{\circ} 3\right)$. Vip. II.

${ }^{50}$ ) Nous reviendrons sur cet exemple au cours du commentaire de

(51) Ibid., 33e pilier, 7e tranche $\left(\operatorname{dossier} \mathrm{n}^{\circ} 5\right)$.

(52) Ibid., 33e pilier, 2e tranche (dossier $\left.\mathrm{n}^{\circ} 6\right)$. 
d'un massif de protection (cas $\mathrm{n}^{\circ} 1$ ), massif riche dissimulé par un massif pauvre (cas $\left.n^{\circ} 2\right)$. Dans d'autres cas $\left(n^{\circ} 4\right)$ un événement imprévisible (éboulement par exemple) a pu survenir, à moins que le chantier n'ait été arrêté au moment de l'abandon de la mine. Mais, dans le cas $n^{\circ} 7$ l'espoir de trouver en profondeur des minerais plus riches a pu jouer.

Enfin, nous avons jusqu'ici raisonné en fonction de l'exploitation des minerais de cuivre. Mais il ne faut pas oublier qu'à Aljustrel, comme le montre Vip. II, à côté de putei aerarii il y avait des putei argentarii, c'est-à-dire des concessions où l'on recherchait en priorité l'argent. Cela peut donc parfois expliquer que de riches minerais de cuivre aient été négligés.

\section{$2^{\circ}$ Les métaux nobles}

On sait que, dans les gisements pyriteux du Sud-Ouest de la Péninsule, à la base du chapeau de fer, il est courant de rencontrer des minerais complexes associés aux métaux nobles, or et argent (53) ; les Algares n'ont pas dû échapper à la règle et les Anciens ont dû développer leurs chantiers dans ces zones (54); d'autre part, dans le gisement d'Aljustrel il y avait de la galène qui, selon le rapport de Leitão, avait une teneur de $1000 \mathrm{~g}$ d'argent par tonne de plomb (55). Enfin l'exploitation de l'argent est attestée par Vip. IL

\section{$3^{\circ}$ La question $d u$ fer}

Les déchets de minerais rencontrés dans les scories sont des témoins indiscutables de la nature des minéralisations recherchées

(53) Cf. Domergue, Mines, Première partie.

(54) Bien que n'affleurant pratiquement pas et quoiqu'il ait subi plutôt une action chimique qu'une véritable érosion physique, le gisement de Moinho possède un chapeau de fer et le minerai sous-jacent renferme des solutions de cuivre, riches en or et en argent; deux échantillons analysés en 1971 par le Laboratoire de la Mine d'Aljustrel, renfermaient respectivement 329 et $364 \mathrm{~g}$ d'argent, 5,2 et 3,7 $\mathrm{g}$ d'or par tonne.

(55) Leitã O, Relatório, p. 476. Estácjo da Veiga, A tabula de bronze, p. 8 signale une teneur de $725 \mathrm{~g}$ Ag par tonne de plomb. On peut considérer que la scorie $\mathrm{n}^{\circ} 354$ (voir infra note 59) est une scorie plombeuse, provenant de la métallurgie de minerais d'argent. 
par les Anciens; or nous avons aussi remarqué de nombreux fragments de roche contenant des oxydes de fer - en particulier un grès et un schiste ferrugineux -, minerais pauvres dont il est logique de penser que les fondeurs se sont débarrassés comme ils ont fait des minerais de cuivre déjà mentionnés. Il semblerait donc qu'à Aljustrel on ait exploité des minerais de fer; de nombreux arguments plaident en faveur de cette hypothèse, et d'abord la richesse du «gossan» en oxydes de fer: en particulier celui des Algares renfermait, selon la notice du catalogue de Monteiro et Barata (56), de grandes masses de limonite traversées par des travaux antiques. Ensuite les scories d'Aljustrel (fîg. 9) constituent une masse énorme: elles couvrent une surface de $444500 \mathrm{~m}^{2}$ et l'épaisseur du crassier atteignait par endroits $10 \mathrm{~m} \mathrm{(57)}$; en admettant une épaisseur moyenne de $3 \mathrm{~m}$, on obtient un total de $1333500 \mathrm{~m}^{3}$, soit au moins 3000000 tonnes; même si l'on suppose que les débris de fours et de constructions diverses constituent le $1 / 3$ de ce total, il reste quelque 2000000 tonnes de scories (58) ; c'est beaucoup pour une mine où l'on n'aurait exploité que du cuivre et de l'argent. En outre ces scories sont pour la plupart très pauvres en cuivre. Sur le plan de 1867 (fig. 24) sont indiquées les zones des crassiers où les scories, tachées de vert par les carbonates, sont sûrement des scories cuivreuses. Mais ces zones (secteurs $\mathrm{d}$ 'Azinhal et $\mathrm{du}$ vallon dos Feitais) sont trop réduites pour qu'on puisse sur cette seule indication distinguer les scories provenant de la métallurgie du cuivre et les autres, d'autant que nous avons vu des scories de ce type disséminées dans l'épaisseur de crassiers sur la pente à l'est des Algares. Sans doute on a dû utiliser des oxydes de fer comme fondants pour éliminer la silice (quartz) des minerais de cuivre, mais les analyses de plusieurs échantillons de scories antiques d'Aljustrel révèlent le plus souvent soit l'absence totale de cuivre, soit des teneurs très basses, incompatibles

(56) Monteiro-Barata, Exposição, p. 96.

(57) Freire de Andrade, A lavra romana, p. 276.

(88) Le chiffre de 450000 tonnes avancé par Allan, A mineração em Portugal, p. 11, est nettement inférieur à la réalité. Vu la surface couverte et la densité des scories, même en n'admettant qu'une épaisseur d'un mètre, on obtient un chiffre d'au moins 1000000 tonnes. 
avec le niveau des techniques métallurgiques de l'époque ( $\mathrm{Si} * * * * * * 59)$, alors que les teneurs en fer et en silice ainsi que les proportions entre ces deux éléments sont remarquablement cohérentes, s'agissant

Ánalyses de scories antiques, effectuées par J. Bourhis (pour les numéros et les signes, voir supra, note 43):

\begin{tabular}{|c|c|c|c|c|c|c|c|c|c|c|c|c|}
\hline No & $\mathrm{Cu}$ & Sn & $\mathrm{Pb}$ & As & $\mathrm{Sb}$ & Ag & $\mathrm{Ni}$ & $\mathrm{Bi}$ & $\mathrm{Fe}$ & $\mathrm{Zn}$ & Mn & $\mathrm{Au}$ \\
\hline 352 & - & 0,001 & 0,001 & 0,001 & - & - & 0,08 & - & ++ & - & 0,08 & \\
\hline 353 & 0,25 & 0,008 & 0,05 & 0,005 & 0,015 & 0,001 & 0,005 & 0,001 & ++ & $<0,001$ & 0,05 & - \\
\hline 354 & 0,35 & 0,10 & 0,60 & 0,10 & 0,10 & 0,008 & 0,001 & - & $++t$ & 0,10 & 0,008 & $<0,001$ \\
\hline 355 & 1 & 0,007 & 0,10 & 0,005 & 0,06 & 0,002 & - & 0,001 & ++ & 0,10 & 0,05 & $<0,001$ \\
\hline 356 & 0,40 & 0,01 & 0,05 & 0,01 & 0,01 & 0,05 & 0,001 & - & +++ & 0,01 & 0,01 & - \\
\hline 357 & 0,40 & 0,01 & 0,05 & 0,01 & 0,01 & 0,005 & 0,005 & 0,005 & $++t$ & 0,50 & 0,01 & $<0,001$ \\
\hline
\end{tabular}

Les échantillons proviennent de Mangancha (couche du 1er s. av. J.-C.: $\mathrm{n}^{\text {os }}$ 352-353), de l'habitat du chapeau de fer des Algares (couche du ler s. ap. J.-C.: $\mathrm{n}^{\circ}$ 354; couche du lile s. ap. J.-C.: $\mathrm{n}^{\circ}$ 355), des crassiers romains d'époque impériale de Feitais ( ${ }^{\text {os }} 356$ et 357 ).

Analyses de scories antiques, effectuées par le laboratoire des mines

d'Aljustrel entre 1914 et 1941: Si

\begin{tabular}{|c|c|c|c|c|c|c|c|c|c|c|}
\hline $\mathrm{N}^{\circ}$ & $\mathrm{Cu}$ & $\mathrm{Fe}$ & $\mathrm{SiO}_{2}$ & $\mathrm{~S}$ & As & Al & $\mathrm{Zn}$ & $\mathrm{Pb}$ & $\mathrm{CaO}$ & $\begin{array}{l}\text { Provenance } \\
\text { précise }\end{array}$ \\
\hline 1 & 0,30 & 44,77 & 31,50 & 1,03 & tr. & 1,05 & tr. & tr. & 0,10 & $?$ \\
\hline 2 & - & 41,48 & 18,50 & - & - & - & - & - & - & Valdoca \\
\hline 3 & 0,58 & 42,58 & 31,50 & 2,60 & - & 3,34 & 0,09 & - & - & $?$ \\
\hline 4 & - & 42,74 & 14,00 & - & - & - & - & - & - & Repreza \\
\hline 5 & 0,58 & 42,58 & 34,75 & 2,60 & - & - & - & - & - & $?$ \\
\hline 6 & 0,26 & 41,61 & 22,00 & 2,39 & 0,20 & - & - & - & - & Telheiro \\
\hline 7 & - & 43,51 & 14,50 & - & - & - & - & - & - & $?$ \\
\hline 8 & - & 49,91 & 7,60 & - & - & - & - & - & - & $?$ \\
\hline
\end{tabular}

Si l'on accepte le critère établi par Salkield, Ancient slags, p. 95 (une scorie qui contient plus de $0,50 \% \mathrm{Cu}$, peu de plomb et peu d'argent est une scorie cuivreuse; dans le cas contraire, c'est une scorie plombeuse), nous pouvons considérer:

— comme scories cuivreuses les nos 355 à 357 (série de Rennes), 3 et 5 (série Aljustrel)

- comme scorie plombeuse le $\mathrm{n}^{\circ} 354$ (série de Rennes) (voir supra, note 55).

Et les autres? Vu l'absence totale ou quasi-totale de cuivre et de plomb, les nos 352 (série de Rennes) 2, 4, 7 et 8 (série d'Aljustrel) peuvent fort bien provenir de la métallurgie d'oxydes de fer. 
de rebuts de la métallurgie d'oxydes de fer riches en silice comme le sont ceux des chapeaux de fer, en particulier aux Algares. La question de la production du fer à Aljustrel dans l'antiquité reste donc posée, comme d'ailleurs dans tous les sites analogues du Sud-ouest de la Péninsule.

Ainsi nous considérons que, dans l'antiquité, on a produit à Aljustrel du cuivre, de l'argent, probablement de l'or et peut-être du fer.

\section{La chronologie des travaux. L'histoire du site}

Le témoignage le plus ancien est fourni par un tesson de campaniforme, qui fait remonter les débuts de l'exploitation des Algares à l'Enéolitique, plus précisément à l'aube du Ilème millénaire avant notre ère $\left({ }^{60}\right)$. Le cuivre est alors le métal recherché, ainsi, peut-être, que l'or natif.

La pointe de flèche en cuivre signalée par Estácio da Veiga et dont l'étude a été reprise par Schubart semble dater du Bronze 1 (1500 à 1100) du Sud-Ouest (61) tandis que les maillets à rainure provenant des Algares remontent au moins au Bronze final, à l'image des fragments de vases de Mangancha présentés ci-dessous. Sans doute, au cours des sondages réalisés en 1967 et 1969 à Mangancha (62), la couche correspondant à cette époque ne renfermait-elle aucun fragment de carbonate de cuivre, mais ces sondages ne sont pas assez nombreux par rapport à la superficie de l'oppidum pour être représentatifs. Aussi pensons-nous que, dès ces époques, l'activité minière a existé tant aux Algares qu'au filon São João, près de Mangancha. On a alors exploité le cuivre, peut-être aussi l'or et l'argent.

(60) Domergue-Freire de Andrade, Sondages à Aljustrel, p. 108-111 et fig. 9-10.

(61) Estácio da Veiga, Antiguidades, p. 127 et fig. 9, p. 124; SChubart, Die Kultur, p. 71, 245-246, n. ${ }^{\circ} 339$ et pl. 34.

(62) Domergue-Freire de Andrade, Sondages à Aljustrel, p. 106-107. Mangancha est un oppidum situé près de la mine S. João. Il a été occupé au moins du Bronze final au 1er siècle av. J.-C. 
Dans la couche supérieure de Mangancha, datée du 1er siècle avant J.-C., apparaissent plusieurs fragments de malachite provenant de São João. Si l'on en croit le témoignage d'une scorie de Mangancha ( ${ }^{63}$ ), dès cette époque la production de fer pourrait s'être ajoutée à celle des autres métaux.

Mais à partir de l'époque augustéenne, c'est près du gisement des Algares que se concentre l'habitat: au Ilème siècle, on l'appellera le Metallum Vipascense. Tant l'étude du matériel recueilli dans les tombes de la nécropole de Valdoca ${ }^{(64)}$ que les sondages effectués dans les ruines des constructions antiques existant sur le «gossan» des Algares ( $\left.{ }^{65}\right)$ prouvent l'occupation du site et la permanence de l'activité minière et métallurgique au moins jusque dans la deuxième moitié du IIlème siècle de notre ère. De même, dans les scories où, à part les tegulae et les briques de fours, le matériel céramique est rare, nous avons recueilli des fragments de vases du $1 \mathrm{er}$ et du Ilème siècle de notre ère. La mine produit alors du cuivre, de l'argent ${ }^{66}$ ), peut-être aussi de l'or et, croyons-nous, du fer.

Par ailleurs l'histoire de cette période est illustrée d'un côté par les deux tables de bronze, Vip. I et Vip. //, trouvées dans les scories, de l'autre par la base en marbre mentionnant un procurateur et dont le texte est rappelé dans l'appendice. Les premières, y compris selon nous Vip. /, datent de l'époque d'Hadrien. L'étude de la seconde, où ledit procurateur est appelé restitutor metallorum nous a amené à la dater de 173 et à y voir un témoignage de reconnaissance des colons de Vipasca au procurateur anonyme qui aurait remis en activité la mine et ses installations, ravagées par le raid maure de 172 dans le Sud de la Péninsule ( ${ }^{67}$ ).

Enfin, les ruines de l'édifice situé sur la pente, à $150 \mathrm{~m}$ à l'est des Algares, et connu sous le nom de «Maison du Procurateur»

(63) Voir supra, notes 55 et 59.

(64) AlarCã O, $O$ espólio, p. 7-8.

(65) Domergue-Freire de Andrade, Sondages à Aljuslrel, p. 104-105.

(66) Peut-être a-t-on recherché l'argent avec moins d'acharnement que le cuivre, ce qui a pu pousser Hadrien à prendre les dispositions en faveur de l'exploitation des putei argentarii, contenues dans Vip. II, 2.

(67) Pour plus de détails, voir Domergue, Mines. 
ont fourni, outre une grande abondance de T. S. arétine, galloromaine et hispanique, quelques fragments de T. S. claire estampée qui prouvent l'occupation du site à la fin du IVème et au début du Vème siècle de notre ère: il est entouré de scories, mais cela suffit-il pour conclure à la permanence de l'activité minière et métallurgique à cette époque?

\section{E. Conclusion}

On ne dira jamais assez l'importance d'Aljustrel dans l'histoire des mines antiques de la Péninsule Ibérique. On y trouve un des plus anciens témoignages d'une occupation susceptible d'être mise en relation avec l'extraction du cuivre. Elle est, au même titre que Riotinto (H 40), Sotiel Coronada (H 20) ou Cabezas del Pasto (H 39), un modèle de mine romaine avec son travers-banc d'exhaure et ses indices d'exploitation rationnelle. Elle a fourni les deux textes les plus riches sur l'organisation fiscale des travaux miniers sous le Haut-Empire, sur les modalités pratiques de mise en valeur d'un gisement, enfin sur la règlementation en usage dans une mine impériale et dans la bourgade qui en dépendait.

Cette bourgade, Vipasca, nous en connaissons l'emplacement. C'est à Valdoca, à l'ouest des Algares. Alors même qu'elle est encore ensevelie, nous savons par Vip. I qu'on y trouvait forum, thermes, école, boutiques, etc. Quelle illustration la fouille du site n'apporterait-elle pas à ces textes de loi! Quels compléments aussi, car, quelque intéressantes que soient les deux tables de bronze, elles sont fragmentaires et laissent beaucoup ignorer de la vie quotidienne à Vipasca.

Or, au moment où nous écrivons, nous savons que le site est menacé p r la construction de bâtiments industriels. Par bonheur, l'Institut Portugais du Patrimoine Culturel, alerté, a pris des mesures pour sauver Vipasca. Et nous nous en réjouissons, car, n'en doutons pas, pour les raisons que nous venons d'exprimer, il faut sauver Vipasca. 


\section{APPENDICE}

Nous rassemblons dans cet appendice les textes épigraphiques et le matériel archéologique permettant d'éclairer l'histoire du site et d'illustrer l'activité de ses mines.

\section{Epigraphie}

Elle comprend des textes bien connus:

- Vip. I et Vip. II, dont nous donnons le texte et une traduction, accompagnés d'un commentaire, dans la deuxième partie de cette étude;

- une dédicace gravée sur une base de marbre trouvée près de la mine en 1907 et dont voici le texte (68):

Lib (ou Tib) [...] iio, Aug(usti) Ub(erto), proc(uratori) diligenl ti[ss]imo et amantissimo, ralti[onaliu]m uicar(io), homini op/timo et iu[stissi]mo, restitu/tori metallo[r]uml, coloni Aug(usti) n(ostri) metalli Vipas/censis statuam cum basi de suol libenter posuerunt ii I qui infra scripti suntj, dedicante ipso.fT. Iunius

Dedi]cata [.........]/Cn. Claud[io...].

' - une inscription funéraire (69) : D(is) M(anibus) S(acrum)/ $M^{\wedge}$ destianus $I$ an(norum) $p$ (lus) m(inus) $X /$ h(ic) s(itus) e(st.) $S$ (it) t(ibi) t\{erra) l\{euis). I P(ublius) Aelius Modes/tus filio pien[tissimo.

L'inscription, trouvée en 1905, à Valdoca, sur le versant occidental des Algares, provient sans doute de la nécropole dont J. et A. de Alar cão ont publié le matériel $\left({ }^{70}\right)$.

(68) D'après Wickert, Bericht, p. 9-12 et Lambrino, Catalogue, p. 130, $n^{\circ} 44$.

(69) D'après CuQ, Notes d'épigraphie, p. 311 et 312, et LAM B R INo, Catalogue, p. 131, $\mathrm{n}^{\circ} 45$.

(70) Alarcão, O espólio, p. 7-109.

Conimbriga, 22 (1983), 5-193 
Ajoutons-y des graffiti, gravés sur des vases provenant de la nécropole de Valdoca. Ils nous donnent les noms d'habitants de Vipasca. Ce sont Palentia ( $\left.{ }^{71}\right)$, Tancus $\left({ }^{72}\right)$ auxquels nous joignons les deux suivants, inédits, gravés sur des urnes cinéraires: L. [Q]uintil[l]a / h(ic) s(ita) e(st) (73) et L(ucius) $\mathrm{F}(\mathrm{ou}$ X)irius Calatius / / S[...] / An[...](*).

\section{Matériel archéologique}

Nous ne revenons pas sur le matériel céramique qui a été soit publié antérieurement par J. et A. de Alarcão ${ }^{(75)}$ soit signalé par R. Freire de Andrade et nous-même $\left({ }^{76}\right)$. De même, malgré la révision qu'il nécessiterait, nous laissons de côté le matériel provenant d'Aljustrel (monnaies, lampes, T. S. arétine, T. S. gallo-romaine, verre) déposé soit au Musée National d'Archéologie de Belém, soit aux Services Géologiques du Portugal à Lisbonne, soit au Musée d'Aljustrel, car il n'apporte rien de nouveau pour la chronologie (77), mises à part quelques monnaies de Maxence (306312) et Valentinien I (364-375) ou II (375-392).

En revanche, nous mentionnons une pointe de flèche de l'Âge $\mathrm{du}$ Bronze et nous présentons deux tessons recueillis en surface sur les pentes de Mangancha, deux autres trouvés par nous mêlés aux scories, enfin deux fragments de T. S. claire provenant de la Maison du Procurateur.

Les objets présentés ci-dessous sont conservés au Musée d'Aljustrel sauf le $\mathrm{n}^{\circ} 7$ (Museu Nacional de Arqueologia e Etnologia, de Belém) et le n 6 (Serviços Geológicos de Portugal).

(71) Alarcão, $O$ espólio, p. 60.

(72) Et non TANCIS, comme a lu Ala a RCão, ibid., p. 62.

(73) Museu Nacional de Arqueologia e Etnologia, de Belém, inv. n 15767.

(74) Ibid., inv. $n^{\circ}$ 15759. Sur une autre urne (inv. $n^{\circ} 15$ 758) on lit

[....... ] D M S / hedera.

(75) Alarcão, $O$ espólio, p. 7-109.

(76) Domergue-Freire de Andrade, Sondages à Aljustrel, p. 104-115.

(77) Ce matériel a été partiellement publié par Veiga FERREIRA-Freire de Andrade, Marcas, p. 1-6.

Conimbriga, 22 (1983), 5-193 
Mangancha (fig. 34)

Outre les renseignements déjà fournis par la note sur les sondages archéologiques effectués à Mangancha, voici deux fragments intéressants trouvés en surface.

1 - Grande coupe carénée, à bord retourné vers l'extérieur. Pâte

grossière, de couleur noir marron, renfermant un abondant dégraissant de quartz. Lissé intérieurement et extérieurement. Le profil caractérise le Bronze final dans le Sud et le Sud-Ouest de la Péninsule, ainsi que dans le Levant $\left({ }^{78}\right)$. Nous avons trouvé des vases de ce type à Gerro Muriano (GO 39), à Tharsis (H 12), etc.

2 - Fragment de la panse d'une amphore. Pâte rouge brique,

friable. Profil caractéristique indiquant l'élargissement du récipient vers le bas.

Amphore du type phénicien fréquent dans les gisements andalous du Vllème au Vème siècle avant notre ère: Tharsis ( $\mathrm{H} \mathrm{12}$ ), Riotinto (H 43), Huelva, Torre del Mar, etc.

Algares (fig. 34)

Matériel recueilli parmi les scories, dans le grand crassier situé à $500 \mathrm{~m}$ à l'est des Algares.

3 - Fragment de la carène d'une patère Drag. 15/17 en T. S.

gallo-romaine. Pâte rosée, dure. Vernis marron chocolat, mat à brillant.

Date: deux derniers tiers du 1er siècle après J.-C. $\left({ }^{79}\right)$.

4 - Fragment du bord d'un bol en T. S. claire A, du type

Hayes 14 A. Vernis rouge, clair à l'intérieur, plus sombre à l'extérieur. Date: milieu du Ilème siècle $\left({ }^{80}\right)$.

(78) Arteaga-Serna, Influjos, p. 742-743 et note 9. Pour le Portugal, un catalogue de la céramique lissée du Bronze final a été récemment publié par SPINDER et alii, Le monument, p. 91-153: on y trouve de nombreux profils comparables au nôtre; on retiendra plus spécialement les figures 14 et $19 \mathrm{~d}$, e, dont les profils sont ceux de vases dépourvus de décoration.

D'autres vases de ce type, mis au jour par nos sondages de Mangancha, seront prochainement publiés.

(79) Vernhet, Notes, pi. VI.

$\left.{ }^{80}\right)$ Hayes, Late Roman Pottery, p. 39-41. 


\section{«Maison du Procurateur» \\ T. S. claire D}

5 - Fragment de fond de plat, dont le centre est décoré de six cercles concentriques (type Hayes 29).

Style Hayes A II: fin IVème-début Vème siècle ( $\left.{ }^{81}\right)$.

6 - Fragment de fond de plat portant un décor estampé comportant plusieurs motifs en disposition radiale: palme avec barbes en relief dans un cachet en creux (dessin assez proche du type Hayes 3); grille proche du type Hayes 71; quatre cercles concentriques (type Hayes 27).

Style Hayes A II, daté de la fin du IVème et du début du Vème siècle (350-420) ( $\left.^{\mathrm{S} 2}\right)$.

\section{Intérieur de la mine}

7 - Pointe de flèche à longue soie, en cuivre arsenical. L. : $11,9 \mathrm{~cm}$.

Datée par Schubart du Bronze I du Sud-Ouest, soit de la deuxième moitié du Ilème millénaire avant notre ère $\left({ }^{83}\right)$.

Objects et instruments se rapportant à Vart des mines ou à la métallurgie antique

Plusieurs de ces objets ont été publiés avec d'excellentes photographies dans l'article de Viana, Freire de Andrade et Veiga Ferreira, Minerações romanas, cité dans la bibliographie. Ils sont conservés au Musée d'Aljustrel (n ${ }^{\text {os }} 26$ et 27), au Museu Nacional de Arqueologia \& Etnologia, de Belém (nos 8, 9, 11, 15, 16, 19 à 23, 28, 29, 31), aux Services Géologiques du Portugal à Lisbonne $\left(10,12,13,17,18,24,25,30,32\right.$ à 38). Le ${ }^{\circ} 14$ est perdu.

8 et 9 - Deux maillets à rainure en roche verte. L. : $20 \mathrm{~cm}$ et

$18,5 \mathrm{~cm} . \mathrm{N}^{\circ}$ d'inventaire: 10.280 A et B.

(81) H y y s, Late Roman Pottery, p. 218-219, 236.

(82. Ibid., p. 218-219, 235, 241-242.

(83) Estácio da Veiga, Antiguidades, p. 127 sq. et fig. 9, p. 124. SChUbart, Die Kultur, p. 71, p. 245-246, n 339 et pl. 34. 
10 — Pic en fer oxydé. L.: $17 \mathrm{~cm} ; 1$ max.: $5,5 \mathrm{~cm}$; ép.: $3 \mathrm{~cm}$. La partie pic est légèrement recourbée, la partie marteau quelque peu écrasée.

11 - Pic en fer très oxydé. L.: $28 \mathrm{~cm} ; 1$ max.: $5,5 \mathrm{~cm}$.

12 - Cadres d'étayage de puits, en chêne, constitués chacun de quatre éléments.

13 - Elément en chêne vert pourvu d'une encoche à chaque extrémité. L.: $136 \mathrm{~cm}$. Elément de boisage d'une galerie?

14 - Quatre treuils comprenant tambour, poulies et supports trouvés dans la mine des Algares à $112 \mathrm{~m}$ de profondeur - (fig- 33) r).

15 — Poulie en chêne vert. Diam. moyen: $18,5 \mathrm{~cm}$; ép.: $5 \mathrm{~cm}$. Trou d'axe irrégulier; gorge abîmée (fig. 14).

16 - Poulie en chêne vert, plus régulière que le $n^{\circ} 15$. Diam.: $22 \mathrm{~cm}$. Gorge bien marquée. Trou d'axe ovale (fig. 14).

17 - Fragments de câble en sparte, à trois torons; diam.: 4,5 cm.

18 - Couffin en sparte à deux poignées; 1 . (à plat: $48 \mathrm{~cm}$; h. : $31 \mathrm{~cm}$.

19 - Echelle rudimentaire formée par un tronc de chêne vert quelque peu tordu; prenait appui sur la fourche. H.: $240 \mathrm{~cm}$, diam.: $20 \mathrm{~cm}$. Six encoches (fig. 13).

20 à 22 - Trois fragments d'échelles du même type que le $\mathrm{n}^{\circ} 19$.

L. respective: $85 \mathrm{~cm}$ (quatre degrés); $35 \mathrm{~cm}$ (un degré); $80 \mathrm{~cm}$ (trois degrés).

23 - Echelle à pied fourchu, brisée en deux tronçons. L. totale: $185 \mathrm{~cm}$ (cinq encoches).

24 - Echelle en chêne vert à trois encoches. L.: $100 \mathrm{~cm}$; diam. $15 \mathrm{~cm}$.

25 - Ecope ou plateau en bois, de section trapézoïdale, sans manche, d'une seule pièce. L.: 29 cm.; L: $16 \mathrm{~cm}$; h.: $7 \mathrm{~cm}$.

26 - Ecope ou plateau en chêne vert, de section trapézoïdale, pourvue d'un manche, le tout d'une seule pièce. L.: $35 \mathrm{~cm}$; L: 13,5 cm; h.: $5 \mathrm{~cm}$ (fig. 16).

${ }^{84}$ C'est cet ensemble qui est publié par VIan a et alii, Minerações romanas, p. $80-82$. 
27- Racloir en chêne vert, demi-circulaire; $1 .: 17 \mathrm{~cm}$; h.: $6,5 \mathrm{~cm}$. Il porte un trou pour le manche, qui était assujetti par une cheville (fig. 16).

28 - Vase en bronze à fond légèrement bombé et muni d'un rebord plat. Très mauvais état. Deux trous sont visibles sur un côté, assez proches l'un de l'autre. Les dimensions devaient être les mêmes que celles de l'exemplaire $n^{\circ} 29$.

Ce vase a-t-il servi à l'épuisement? Etait-il suspendu par les deux trous au câble en spart dont on voit les restes à l'intérieur?

29 - Vase en bronze de même type que le $\mathrm{n}^{\circ} 21$, mais mieux conservé. H.: $21 \mathrm{~cm}$; diam.: $33,5 \mathrm{~cm}$; ép. de la feuille de bronze: 0,1 cm (fig. 15). $\mathrm{N}^{\circ}$ d'inventaire Belém: 17864 .

30 - Trois attaches en bronze destinées à accrocher l'anse d'un seau. Il est vraisemlable que les parois de ces seaux étaient en bois.

31 - Rigole faite d'un tronc de chêne vert creusé. L.: $200 \mathrm{~cm}$; 1.: 22 à $25 \mathrm{~cm}$. Profil en auge; dimensions intérieures; $1 .: 15 \mathrm{~cm}$; prof.: $6 \mathrm{~cm}$ (fig. 13).

32 - Rigole en chêne vert. L.: $63 \mathrm{~cm}$; L: $14 \mathrm{~cm}$.

33 - Massue en chêne vert. L. : $31 \mathrm{~cm}$.

34 - Bonnet en spart. H.: $16 \mathrm{~cm}$; 1 . (à plat): $25 \mathrm{~cm}$.

35 — Semelle d'espadrille en spart. L. : $25 \mathrm{~cm}$.

36 - Embout de soufflet en fer oxydé; 1 . max.: $13 \mathrm{~cm}$.

37 — Lingot de cuivre rectangulaire; retassures en surface.

L.: $28 \mathrm{~cm}$; $1 .: 17 \mathrm{~cm}$; ép.: $3 \mathrm{~cm}\left({ }^{85}\right)$.

Trouvé dans les scories, comme le $\mathrm{n}^{\circ} 38$.

38 - Poids en plomb de forme elliptique. Grand axe: $18 \mathrm{~cm}$; petit axe: $14 \mathrm{~cm}$; ép.: $2,5 \mathrm{~cm}\left({ }^{86}\right)$.

Il s'agit d'un poids (on voit sur une face les points d'attache de la poignée) et non d'un lingot.

(85) Analyse (d'après VianA et alii, Minerações romanas, p. 86):Cu: 95,50; As: 1,14; S: 0,80; Fe-fAl: 0,23; Bi: 0,23; Si02: 0,65; Ni: tr.; Zn: tr.; Sn: 0,82; Sb: 0,21 .

(86) Analyse (Ibid., p. 86): Pb: 99,47; Si02: 0,30. 
LIVRE II

\section{LES TABLES DE BRONZE DE VIPASCA}

Notre intention n'est pas de donner ci-dessous une édition critique des textes gravés sur les deux tables de bronze $\left({ }^{x}\right)$ trouvées dans les crassiers de la mine d'Aljustrel. Néanmoins, alors que l'établissement $\mathrm{du}$ texte même de Vip. $/ /\left(^{2}\right)$ ne pose pas de problème, l'état de Vip. I nous a conduit à examiner l'original de près. Nous avons ainsi fait un certain nombre d'observations matérielles que nous rassemblons en tête de notre étude. Pour les deux tables, nous adoptons, à l'exclusion de quelques modifica-

P) Nous conservons le terme «table de bronze» malgré les résultats d'une analyse de la table de Vip. /, mentionnés dans Companhia de Mineração Transtagana, Gerência de 1876 (dactylographié), Archives des mines d'Aljustrel, p. 21. Voici ces résultats (en pourcentages): $\mathrm{Cu}$ 76,4; $\mathrm{Sb}$ 10,2; $\mathrm{Pb}$ 11,47; $\mathrm{Ag} \mathrm{o,008;} \mathrm{Zn} \mathrm{o,6;} \mathrm{Fe} \mathrm{1,15;} \mathrm{As,} \mathrm{S,} \mathrm{GO,} \mathrm{Bi:} \mathrm{traces} \mathrm{(=} \mathrm{différence} \mathrm{o,17).}$ Le commentaire donné par le document est que, vu la composition de l'échantillon, le métal dont est faite la table provient de la fusion de minerai d'Aljustrel. On remarquera en tout cas l'absence totale d'étain. Il s'agit donc de cuivre très impur, ou plutôt d'un alliage constitué principalement de cuivre, d'antimoine et de plomb. Mais en toute rigueur ce n'est pas un bronze.

Nous ignorons s'il existe une analyse plus moderne de la table de Vip. I et si la table de Vip. II a elle-même été analysée.

(2) Nous adoptons les abréviations de D'Ors, Epigrafia, p. 71 sq. Vip. I désigne la table qui fut trouvée la première (1876), Vip. II celle qui a été découverte en 1906.

Pour les abréviations bibliographiques, on se reportera à la bibliographie donnée in fine.

Conimbriga, 22 (1983), 5-193 
tions de détail qui seront signalées, le texte qui est donné dans Girard et Senn, p. 78-87.

Nous indiquons à la fin de notre étude la bibliographie que nous avons utilisée pour étayer notre commentaire. Par ailleurs des recherches récentes en archéologie minière, la connaissance que nous avons du site d'Aljustrel, enfin les conclusions que nous tirons de nos travaux sur les mines antiques de la Péninsule Ibérique ( $\left.{ }^{3}\right)$ nous font proposer une interprétation de Vip. II différente de celle qui est habituellement adoptée.

La présentation des textes appelle quelques remarques. Dans le texte latin, les mots entre crochets droits sont les restitutions des lacunes; le développement des abréviations a été placé entre parenthèses; les compléments dus à des erreurs ou à des oublis $\mathrm{du}$ lapicide se trouvent entre crochets triangulaires. Dans le texte français, les mots mis entre parenthèses sont destinés à rendre plus clair le sens du texte.

Dans Vip. /, nous avons numéroté les chapitres de 1 à 9 , et, dans chaque chapitre, les phrases à partir de 1. Ainsi Vip. /, 3, 1 désignera la phrase 1 du chapitre 3 de Vip. I.

Dans Vip. II, nous avons adopté la division en paragraphes que suit d'Ors $\left(^{4}\right)$. C'est en effet celle qui apparaît sur le bronze et qui se marque:

- soit par un espace séparant, sur la même ligne, la fin d'un paragraphe et le début d'un nouveau;

- soit lorsque la fin d'un paragraphe et la fin d'une ligne coïncident, par le décalage vers la gauche du mot initial du paragraphe suivant, si bien que les premières lettres de ce mot mordent sur la marge.

Nous pensons en effet que le regroupement de plusieurs de ces paragraphes, tel qu'il est généralement effectué $\left(^{5}\right)$, peut nuire à l'interprétation du texte, spécialement vers la fin.

(3) Voir notre livre, Les mines de la Péninsule Ibérique à Vépoque romaine (sous presse).

(4) Cf. D'Ors, Lex Metalli, p. 128 et Epigrafia, p. 112-133.

$\left(^{5}\right)$ Voir par exemple Bruns, Fontes, n ${ }^{\circ} 113$; FIRA, I, n $\mathrm{n}^{\mathrm{e}} 104$, p. 498-502; GIRARD-SENN, p. 78-81. 


\section{CHAPITRE PREMIER}

VIPASCA I

Le texte que nous appelons «première loi de Vipasca» (Vip. I) est gravé sur une table de bronze trouvée en mai 1876 dans un crassier de la mine d'Aljustrel. Cette table est conservée au Museu dos Serviços Geológicos de Portugal, à Lisbonne. Elle mesure $785 \mathrm{~mm}$ X $520 \mathrm{~mm}$. Son épaisseur varie de 8 à $13 \mathrm{~mm}$.

La table est opisthographe. Il y a 52 lignes sur chaque face. Dans les deux cas le texte est le même, mais une face renferme, au début, dix lignes qui manquent à l'autre; cette dernière en revanche compte sept lignes de plus à la fin. Le champ épigraphique mesure $600 \mathrm{~mm}$ X $360 \mathrm{~mm}$. Sur la face 1 (e) la hauteur des lettres varie de 7 à $9 \mathrm{~mm}(11$ à $14 \mathrm{~mm}$ pour les titres $)$, sur sur la face 2 elle est de $8 \mathrm{~mm}$ (10 à $12 \mathrm{~mm}$ pour les titres).

La partie droite de la table a été brisée et a disparu. D'autre part le texte avait été gravé de telle façon que, sur les deux faces, c'est la fin des lignes qui manque.

\section{A. La table de bronze et l'inscription}

Il ne semble pas que, depuis les premières éditions de Vip. /, on ait eu recours à l'original pour établir dans ses moindres détails le texte de chacune des deux faces. Pourtant si l'on compare le bronze à l'édition la plus répandue, celle de E. Hübner dans le

(e) Face 1: celle dont le texte commence par centesimae.

Face 2: celle dont le premier mot est majorem. 
Corpus Inscriptionum Latinarum ( $\left.{ }^{7}\right)$, on s'aperçoit qu'elle présente plusieurs erreurs et omissions, le plus souvent, il est vrai, sans importance pour l'établissement et la compréhension du texte. Sans doute l'édition de Rodriguez de Berlanga ( ${ }^{8}$ ) corrigeait-elle par avance nombre de ces imperfections. Mais, vu sa rareté, nous avons jugé bon de présenter quelques remarques sur les points suivants :

\section{Io Vaspect matériel}

Les dimensions: depuis Soromenho $\left({ }^{9}\right)$, on répète le plus souvent $\left({ }^{10}\right)$ que les mesures de la table sont de $72 \mathrm{~cm} 53 \mathrm{~cm}$. En fait les dimensions données par Estácio da Veiga ( ${ }^{n}$ ) doivent être les bonnes, car la partie aujourd'hui visible (12) mesure $780 \mathrm{~mm}$ X $492 \mathrm{~mm}$.

Les trous de suspension: si l'on considère la face 1, il y a en fait:

- dans la marge supérieure, en haut et à gauche, deux trous (diamètre respectif: 11 et $9 \mathrm{~mm}$ ) proches l'un de l'autre;

- dans la marge gauche, vers le bas, à la hauteur de la ligne 46, un troisième trou (diamètre: $14 \mathrm{~mm}$ ).

Par ailleurs, dans la marge inférieure, vers la gauche, un trou de $10 \mathrm{~mm}$ de diamètre semble avoir été bouché ensuite, car, sur la face 2, une protubérance signale l'emplâtre qui le colmate. En

(7) Hübner, Metallum, p. 789-790. Cette édition ne diffère de celle que cet auteur avait déjà publiée dans HüBNER, Lex metalli, p. 167, que sur des points de détail.

(8) Rodriguez de Berlanga, p. 623-830. Pour la reproduction photographique (éch.: 1/2) de chacune des faces, on se reportera aux ouvrages de SOROMENho et D'ESTÂCio da VeIGa (photographies en couleurs) cités ci-dessous ainsi qu'à l'étude de HüBner, Lex Metalli $_{i}$ p. 236 (reproductions en noir et blanc). Voir aussi infra les figures $\mathbf{1 7}$ et $\mathbf{1 8 .}$

$\left({ }^{9}\right)$ Soromenho, p. 4.

(10) En dernier lieu par exemple Thielmann, p. 59.

(u) ESTÁCIO DA Á^EIGA, A tàbula de bronze, p. 10.

(12) La présentation actuelle de Vip. I laisse à désirer: la table est en effet insérée dans un cadre en bois, ce qui empêche qu'on puisse lire la fin de plusieurs lignes, spécialement sur la face 1 . 
outre, plus près du bord inférieur et vers la droite, un autre trou (diamètre: $10 \mathrm{~mm}$ ) n'a été qu'ébauché (profondeur: 1,5 mm).

Mutilations de la partie droite: sur la droite, on a enlevé à la plaque une bande large d'environ $25 \mathrm{~cm} \mathrm{(13)} \mathrm{à} \mathrm{laquelle} \mathrm{s'ajoute,}$ de la ligne 29 à la ligne 41 de la face 1 , une étroite échancrure supplémentaire. Cela n'a pu être fait qu'une fois que la table n'a plus eu d'usage officiel. Alors, ainsi mutilée, elle a dû être soudée à un autre élément, comme semble le prouver le bourrelet apparemment en bronze, long de $69 \mathrm{~cm}$, de section grossièrement rectangulaire $(26 / 31 \mathrm{~mm} \times 13 / 24 \mathrm{~mm})$, qui, appliqué sur la face 2 , dissimule une bande de texte large de 10 à $15 \mathrm{~mm}$ et déborde de la plaque d'une largeur à peu près égale.

\section{$2^{\circ}$ La paléographie}

Les éditeurs antérieurs ont bien marqué les fautes commises par le lapicide qui avait gravé d'abord la face 2, ce qui avait conduit à utiliser l'autre (face 1) pour y graver un texte plus correct. Mais on doit aussi souligner que le ductus des lettres varie d'une face à l'autre: il suffit par exemple de considérer la graphie des $\mathrm{E}$ et la qualité même de la gravure, meilleure sur la face 2 que sur la face 1. Il s'agit donc probablement de deux lapicides différents $\left({ }^{14}\right)$. Le premier (face 2) n'a sans doute pas su lire le texte qui lui était soumis, d'où les lacunes (15) et les fautes d'orthographe facilement décelables. On a eu alors recours à un second graveur (face 1).

$3^{\circ}$

\section{$V$ épigraphie}

L'édition la plus récente du texte de chacune des deux faces est celle de Hübner, dans le Corpus Inscriptionum Latinarum ( $\left.{ }^{16}\right)$.

(13) $15 \mathrm{~cm}$ de texte (Hü BNER, Metallum, p. 789), à quoi s'ajoutait sans doute une marge égale à celle qui existe à gauche, soit $10 \mathrm{~cm}$.

(14) Contra, EsTÁcio dA VEIGA, A tdbula de bronze, p. 15 et Rodriguez DE BERLANGa, p. 652.

(15) La plus importante est après maiorem (1. 1).

(16) HÜ BNER, Metallum, p. 789 et 790. 
Elle renferme malheureusement quelques inexactitudes. Aussi proposons-nous aux figures 20 et 21 une transcription nouvelle $\left({ }^{17}\right)$ que nous accompagnons des commentaires suivants:

Face 1 (fig. 17 et 20)

1. 9: le $M$ de quam comporte un jambage supplémentaire;

1. 22: il faut bien lire $T A M$ et non $T A N$; la dernière jambe oblique du $M$ est incomplète, mais bien nette, et elle n'est pas suivie d'un point.

1. 47: à la fin de la ligne, le $S$ de pondus est partiellement conservé.

Face 2 (fig. 18 et 21)

Les corrections qui portent sur des lettres concernent toutes la fin des lignes:

1. 21 : après $P R O C$, un $\mathrm{V}$ est partiellement visible

1. 28: un second / visible

1. 30: / lisible

1. 36: après le point, on discerne la première haste oblique d'un $A$

1. 38: V partiellement visible

1. 52: $\mathrm{V}$ entièrement lisible.

Par ailleurs, comme le montrent les figures 20 et 21 , les points de séparation ont été placés par les graveurs sur les deux faces sans aucune logique. Mais si l'on compare avec les transcriptions correspondantes de Hübner $\left({ }^{18}\right)$, on constatera que sur ce point l'illustre épigraphiste est loin d'être resté fidèle aux originaux, puisqu'il a ajouté un nombre considérable de points et en a supprimé quelques autres $\left({ }^{19}\right)$.

(17) Comme on l'a déjà signalé à la note 12 , le cadre de bois qui entoure la table rend difficile la lecture de la fin de plusieurs lignes de la face 1, en particulier des lignes 1 à 19, pour lesquelles nous nous en tenons à la lecture de Hübner.

(18) HÜ BNER, Lex Metalli, p. 167, et Metallum, p. 789 et 790.

$\left({ }^{19}\right)$ En revanche les transcriptions de Estácio DA Veiga, A tábula de bronze, fig. hors texte 3 et 4 , et surtout de Rodriguez DE BerLanga, fig. hors texte p. 626 , sont bien plus fidèles. 
Plus importante est la question du chiffre III, qui, selon Soromenho et Estácio da Veiga ${ }^{(20)}$, suivis par Hübner, Rodriguez de Berlanga et bien d'autres encore par la suite $\left({ }^{21}\right)$, aurait été gravé au-dessous des premières lettres de l'ultime ligne du texte de la face 1. On n'a pas manqué d'en conclure que deux autres tables précédaient celle-ci a l'emplacement où elle avait été affichée. Un examen de l'original conduit à plus de prudence, et l'on se reportera pour ce qui suit au calque de l'estampage qui constitue la figure $22\left({ }^{22}\right)$.

Il existe bien en effet, à l'emplacement signalé, trois incisions parallèles et à peu près verticales. Elles sont séparées - la piemièi e de la deuxième et celle-ci de la troisième - par un espace constant de $12 \mathrm{~mm}$. Mais leur hauteur n'est pas identique: de gauche à droite, 7,5 mm, $9 \mathrm{~mm}, 11 \mathrm{~mm}$ respectivement; et, au lieu des hastes bien gravées, de largeur égale et munies d'empattements que l'on trouve dans les chiffres du texte (par exemple aux lignes 17 et 45), il s'agit de simples incisions, pointues à leur extrémité supérieure, plus larges à la base $(1 \mathrm{~mm}$ environ $)$ et qui n'ont pas été retouchées. En outre, il y a, un peu plus haut, dans cette même marge gauche, à la hauteur des lignes 48, 49, 50 et 51, d'autres incisions plus courtes, mais de même foi me et à peu près de même direction, qui font penser que les unes et les autres ont pu être faites accidentellement par les griffes de quelque instrument, et cela dès l'antiquité, sans doute une fois que la table a cessé d'être utilisée officiellement. On remarquera à ce propos, dans ce coin inférieur gauche de la face $1, \mathrm{~d}$ autres indices montrant les mauvais traitements subis par la table: ainsi les petites cavités qui ont écrasé partiellement les premières lettres des lignes 51 et 52 et celles que l'on trouve-au voisinage de la troisième des incisions en question et à quelques centimètres au-dessous de ces dernières.

(20) Soromenho, p. 4; Estácio da Veiga, A tàbula de bronze, p. 13.

(21) HÜbner, Lex Metali, p. 166 et Metallum, p. 793; RodrigueZ de Berlanga, p. 779. Voir aussi D'Ors, Epigrafí, p. 81; Thielmann, p. 59; D. FLACH, Bergwerksordnung, p. 402.

(22) On comparera avec les reproductions signalées à la note 8 . La photographie de notre figure 17 est limitée au champ épigraphique. On distingue cependant en bas et à gauche les trois incisions en question. 
Dans ces conditions, nous hésitons à cioire que ces incisions représentent le chiffre III. Cependant, pour assurer leur interprétation, Estácio da Veiga et Rodríguez de Berlanga se référaient à divers précédents (23). Ainsi Estácio da Veiga citait la lex Rubria, en tête de laquelle est gravé le chiffre 1111 ; mais il y a un abîme entre nos trois incisions et ce chiffre 1111 placé en tête de la table, dans la marge à gauche de la première ligne du texte, et soigneusement écrit, avec ses hastes régulières et de même hauteur, munies d'empattements en haut et en bas( $\left({ }^{24}\right)$.

Nous pensons donc qu'il faut renoncer à voir dans la table qui nous est parvenue la troisième de celles qui constituaient le texte complet de ce règlement minier de Vipasca et que nous devons continuer à ignorer le nombre de celles qui la précédaient tout autant que de celles qui la suivaient.

(23) Estácio da Veiga, $A$ tábula de bronze, p. 13; Rodriguez de BERLANGA, p. 779.

(24) ILLRP, Imagines, n. ${ }^{\circ} \mathbf{3 8 9}$. Voir aussi le chiffre VIIII gravé au-dessus du texte, dans la loi municipale de Tarente (Ibid., n. ${ }^{\circ} \mathbf{3 9 5}$ ). 
B. Le texte et la traduction 


\section{La traduction}

Chap. 1-(Dispositions écrites relatives à l'impôt du) centième (sur le montant des ventes porté) sur la stipulation faite par le banquier

bSar les ventes qui se feront aux enchères à Vintérieur du territoire de la mine de Vipasca, à Vexception de celles que le procurateur des mines fera sur Tordre de VEmpereur, le fermier recevra du vendeur un centième. ${ }^{2}$ Sur le prix des puits que vendra le procurateur des mines, le fermier recevra de Vacheteur un centième. ${ }^{z} S i$ une vente aux enchères a été entreprise mais que tout ait été adjugé en bloc, le vendeur n'en devra pas moins fournir le centième au fermier, à son associé ou à son agent. *Le fermier, son associé ou son agent pourra, s'il le veut, stipuler, ou prendre un gage. ${ }^{5}$ Le fermier, son associé ou son agent réclamera aussi le centième de la somme qui, au cours de la vente aux enchères, aura été reçue (par le banquier). ${ }^{6}$ Celui qui aura mis des marchandises aux enchères et ne les aura pas adjugées, si, dans les dix jours qui suivent, il les vend de gré à gré, n'en devra pas moins payer le centième au fermier, à son associé ou à son agent. ${ }^{7 P o u r}$ toute somme qui, en vertu de ce chapitre de loi, sera dae aa fermier, à son associé ou à son agent, et qui, dans un délai de trois jours à partir du moment où elle sera exigible, n'aura été ni payée, ni acquittée, ni cautionnée, il faudra payer le double. 


\section{Le texte}

(1) Centesimae argentariae stipulationis. Conductor ea [rum dnnditionum, quae per auctio] | nem intra fines metalli Vipascensis fient, exceptis iis, quas proc(urator) metallorum iu|'ssu imp(eratoris) faciet, centesimam a uendito] | re accipito. Conductor ex pretio puteorum, quos proc(urator) metallorum uendet, cen/tesimam ab emptore accipito]. | ${ }^{3} \mathrm{Si}$ instituta auctione uniuersaliter omnia addicta fuerint, nihilo minus uenditor ce[ntesimam conductori socio acto] I riue eius praestare debeto. ${ }^{4}$ Conductori socio actoriue eius, si uolet stipulari au[£ pignus capere liceto. ${ }^{5}$ Conductor $]$ socius actorue eius <eius> quoque summae, quae excepta in auctione erit, centesimam exigito. Qui res sub praecone] | habuerit, si eas non addixerit et intra dies decem, quam sub praecone fuerint, de condici [one uendiderit, nihilo minus con] | ductori socio actoriue eius centesimam $\mathrm{d}\left(\right.$ are) $\mathrm{d}$ (ebeto). ${ }^{7}$ Quod ex hoc capite legis conduct[or $\dot{i}$ socio actoriue eius debebitur], | nisi in triduo proximo, quõm debere coeptum erit, datum solutum satisue factum erit, $d n[$ plum $d$ (are) d(ebeto)]. |

ligne 5/6: [...ab emptore accipito (J. FlaCh, Table de bronze et Bruns, Lex metalli) au lieu de [...ab emptore exigito]

Conimbriga, 22 (1983), 5-193 
Chap. 2 - Dispositions écrites relatives à l'office de crieur public

${ }^{1}$ Celui qui aura loué Voffice de crieur public fournira un crieur public à l'intérieur du territoire (de la mine de Vipasca). ${ }^{2}$ Le fermier réclamera au vendeur $2 \%$ sur toute vente égale ou inférieure à cent (ou cinquante) deniers, 1\% sur toute vente d'un montant supérieur. ${ }^{z}$ Celui qui aura vendu des esclaves aux enchères devra payer au fermier, à son associé ou à son agent une taxe de... deniers par tête s'il en a vendu cinq ou moins, de trois deniers par tête s'il en a vendu davantage. ${ }^{4} \mathrm{Si}$ le procurateur des mines met en vente ou en adjudication des marchandises au nom du fisc, le fermier, son associé ou son agent, devra mettre à sa disposition pour ces marchandises un crieur public. ${ }^{5}$ Celui qui, aura affiché une liste de marchandises, quelles qu'elles soient, à vendre, devra payer un denier au fermier, à son associé ou à son agent. ${ }^{6}$ Sur les puits qu'aura vendus le procurateur des mines, c'est l'acheteur qui devra payer le centième. ${ }^{7}$ Qui, dans les trois jours, n'aura pas payé ce qu'il doit devra payer le double. ${ }^{8}$ Le fermier, son associé ou son agent pourra prendre un gage. ${ }^{9}$ Celui qui aura vendu aux enchères des mulets, mules, ânes, ânesses, chevaux, juments devra payer trois deniers par tête. ${ }^{10}$ Qui aura mis aux enchères des esclaves ou toute autre marchandise et les aura vendus de gré à gré dans les trente jours devra payer au fermier, à son associé ou à son agent la même somme (que s'il les avait vendus aux enchères).

Chap. 3 - (Dispositions écrites relatives à l'exploitation de l'établissement de bains

${ }^{x}$ Le fermier des bains ou son associé, devra, entièrement à ses frais, chauffer tous les jours I'établissement qu'il aura pris à ferme jusqu'à la veille des prochaines calendes de juillet et le mettre, de la première à la septième heure du jour, à la disposition des femmes, et, de la huitième heure du jour à la deuxième heure de la nuit, à la disposition des hommes, à la convenance du procurateur placé à la tête des mines. ${ }^{2}$ Pour ce qui est de l'eau, il devra, comme il convient, en remplir les chaudières en bronze jusqu'au haut de la grenouille et la faire couler abondamment dans la baignoire tant pour les femmes que pour les

Conimbriga, 22 (1983), 5-193 
(2) Scripturae praeconii. ${ }^{1}$ Qui praeconium conduxerit, praeconem intra fines praebe[to. ${ }^{2}$ Conducto ab eo qui uenditionem] $\ddot{i} \mathrm{~L}$ minórenme fecerit, centesimas duas, ab eo qui maiorem $\mathrm{C}$ fecerit, centesimam exig[ito. ${ }^{3}$ Qui mancipia sub praecone ] dederit, si quinque minórenme numerum uendiderit, capitularium in singula capita [ï.., si maiorem numerum nendi] der il. in singula capita III conductori socio actoriue eius dare debeto. ${ }^{4} \mathrm{Si}$ quas [res procurator) metallorum nominé] fisci uen|det locabitue, iis rebus conductor socius actorue eius praeconem praestare debeto. ${ }^{5} \mathrm{Q}\left[\mathrm{u}_{i}\right.$ inuentari]um cuiusque rei uendun $\mid$ dae nomine proposuerit, conductori socio actoriue eius $X$ I d(are) d(ebeto). ${ }^{6}$ PuteorUm, quos proc(urator) metallorum uendiderit, em/ptor centesimam d(are) $\mathrm{d}\left(\right.$ ebeto). ${ }^{7}$ Quod si in triduo non dederit, duplum $\mathrm{d}$ (are) d(ebeto). ${ }^{8}$ Conductori socio actoriue eius pignus cape $<$ re $>$ liceto. | ${ }^{9}$ Qui mulos mulas asinos asinas caballos equas sub praecone uendiderit in $\mathrm{k}$ (apita) $\operatorname{sing}\left(\right.$ ula) $X$ III $\mathrm{d}$ (are) $\mathrm{d}$ (ebeto). ${ }^{10}$ Qui mancipia aliamue quam re[m sub] | praeconem subiecerit et intra dies XXX de condicione uendiderit, conductori socio actoriue eius [idem d(are) d(ebeto)]. |

(3) Balinei fruendi. Conductor balinei sociusue eius omnia sua impensa balineum, [quod ita conductum bit in $\mid \operatorname{pr}$ (idie) $\mathrm{k}($ alendas) Iul(ias) primas omnibus diebus calfacere et praestare debeto a prima luce in horam septim[am diei mulieribus] et ab hora octava | in horam secundam noctis uiris arbitratu procuratoris) qui metallis praeerit. ${ }^{2}$ Aquam in [aenis usque ad] summam ranam hypo|caustis et in labrum tam mulieribus quam uiris

ligne 2: [...Conductor ab eo...] (J. Flach, Table de bronze) au lieu de [...Pro mereede ab eo]

ligne 24: Aquam in [aenis...] (D. Flach, Bergwerksordnung) au lieu de Aquam in [alueum...] 
hommes. *Le fermier fera payer par personne aux hommes un semis de bronze, aux femmes un as de bronze. *Sont exemptés (de ce droit d'entrée) les affranchis et esclaves impériaux qui sont en fonction auprès du procurateur ou qui bénéficient de privilèges, et de meme les impubères et les soldats. ${ }^{5} \mathrm{~A}$ Vexpiration $d u$ bail, le fermier, son associé ou son agent devra rendre en bon état rétablissement de bains et tout le matériel qui lui aura été assigné, à l'exclusion de ce qui aura subi des dommages du fait de sa vétusté. "Tous les trente jours, il devra convenablement laver, frotter et enduire de graisse fraîche les chaudières en usage. ${ }^{7} \mathrm{Si}$ un cas de force majeure empêche pendant quelque temps qu'on puisse convenablement se baigner, le fermier devra déduire (du prix du fermage) une somme proportionnelle à cette durée. ${ }^{8}$ En dehors de ces cas et des travaux qu'il aura par ailleurs effectués pour faire fonctionner ce même établissement de bains, il ne devra rien déduire. ${ }^{9}$ Le fermier n'aura pas le droit de vendre du bois, sauf des parties de branches coupées, qui ne sont pas un bon combustible. ${ }^{10} S^{\prime}$ il agit contrairement à cette prescription, il devra payer pour chaque vente cent sesterces au fisc. "Si cet établissiment de bains n'est pas entretenu comme il convient, le procurateur aura le droit d'infligir chaque fois au fermier jusqu'à 200 sesterces d'amende. ${ }^{12}$ Le fermier aura en tout temps une réserve de bois suffisante pour... jours.

Chap. 4 - (Dispositions écrites relatives au) métier de cordonnier

${ }^{X}$ Qui vendra de ces marchandises, chaussures ou courroies, qui habituellement sont l'affaire des cordonniers, qui plantera ou vendra un clou de semelle ou encore sera convaincu d'avoir vendu, à l'intérieur $d u$ territoire (de Vipasca), n'importe quelle autre marchandise que sont tenus de vendre les cordonniers, celui-là devra payer le double (du prix reçu) au fermier, à son associé ou à son agent. ${ }^{2} L e$ fermier vendra le clou conformément à la loi sur les mines de fer. sIl sera permis au fermier, à son associé ou à son agent de prendre un gage. ${ }^{A} \mathrm{Nul}$ ne pourra réparer des chaussures, sauf s'il s'agit de 
profluentem recte praestare debeto. Conductor a uiris sing(ulis) | aeris semisses et a mulieribus singulis aeris asses exigito. "Excipiuntur liberti et serui [Caes(aris), qui proc(uralori)] in officis erunt uel I commoda percipient, item inpuberes et milites. Conductor socius actorue eius [ balineum et instrument]ta omnia quae | ei

adsignata erunt integra conductione peracta reddere debeto nisi si qua uetustate c [orruptaerunt]. ${ }^{6}$ Aena quibus | utetur lauare tergere unguereque adipe e recenti tricensima quaque die recte debeto. ${ }^{7}\left[. \mathrm{S}^{\prime} \mathrm{i}\right.$ uis maior per aliquod tempus inpedijeni, quo minus lauare recte possit, eius temporis pro rata pensionem conductor reputare debe[io. ${ }^{8}$ Praeter] haec et siquid | aliut eiusdem balinei exercendi causa fecerit, reputare nihil debebit. ${ }^{9}$ Conductori $u$

ligna] nisi ex recisamini|bus ramorum quae ostili ideona non erunt ne liceto. ${ }^{10} \mathrm{Si}$ aduersus hoc quid fecerit, in singul[as uenditiones HS] centenos $\mathrm{n}$ (ummos) fisco $\mathrm{d}\left(\right.$ are) $\mathrm{d}$ (ebeto). | ${ }^{\mathrm{n}} \mathrm{Si}$ id balineum recte praebitum non erit, tum proc(uratori) metallorum multam conductori quo[£i]ens recte praebitum non erit usque I ad HS CG dicere liceto. ${ }^{12}$ Lignum conductor repositum omni tempore habeto, quod diebus. [satis sit]. J

(4) Sutrini. ${ }^{a} Q u i$ calciamentorum quid loramentorumue, quae sutores tractare \&o]lent, uendiderit clauomue cah]ga|rem fixerit uenditaueritue siue quid aliut, quod sutores uendere debent, uendidis[se intra fines conuictus erit, is $\mid$ conductori socio actoriue eius duplum $\mathrm{d}$ (are) $\mathrm{d}$ (ebeto). ${ }^{2}$ Conductor clauom ex lege ferrariarum uendito. 3 Conductorisoci]o | actoriue eius pignus capere licebo. ${ }^{4}$ Reficere calciamenta nulli licebit nisi cu[m sua dominiue

ligne 5: [balineum et instrumenta. (J. FLach, Table de bronze) au lieu do [instrumenta balinei et e] a

ligne 9: [Si uis maior per aliquod tempus impedi]erit (RE) au lieu de [Si qua necessaria refectio impcdi]erit

ligne 21: so [lent, uendiderit...'] au lieu de so[lente, fecerit...] 
Ventretien ou du rapetassage des siennes propres ou de celles de son maître. 5Le fermier sera tenu de fournir toutes les variétés de chaussures, sinon chacun aura le droit d'acheter où il voudra.

Chap. 5 - (Dispositions écrites relatives au) métier de barbier

${ }^{1}$ Le fermier devra jouir d'un droit tel que nul autre ne pourra, pour de l'argent, exercer le métier de barbier dans la bourgade ou dans les territoires de la mine de Vipasca. ${ }^{2} Q u i$ aura exercé ainsi le métier de barbier devra payer au fermier, à son associé ou à son agent... deniers pour chacune des fois où il aura utilisé ses instruments, et ces instruments seront confisqués au profit du fermier. sSont exceptés les esclaves qui auront donné leurs soins à leurs maîtres ou à leurs compagnons. *Les barbiers ambulants qui n'auront pas été mandés par le fermier n'auront pas le droit de raser. ${ }^{5}$ Il appartiendra au fermier, à son associé ou à son agent de prendre un gage. ${ }^{6} Q u i$ l'en empêchera devra, pour chaque fois, lui payer cinq deniers. 'Le fermier prendra à proportion (du travail à faire) un ou plusieurs artisans compétents.

Chap. $6-$ (Dispositions écrites relatives aux) fouleries

IPersonne ne pourra, moyennant salaire, fouler des vêtements neufs ou rapiécés, sinon ceux à qui le fermier, son associé ou son agent en aura loué le droit ou accordé l'autorisation. ${ }^{2} Q u i$ sera convaincu d'avoir contrevenu à ces dispositions devra payer au fermier, à son associé ou à son agent trois deniers par pièce de vêtement. ${ }^{3}$ Le fermier, son associé ou son agent pourra prendre un gage.

Chap. 7 - Dispositions écrites relatives aux exploitants de crassiers et aux carriers

${ }^{r}$ Celui qui, dans le territoire de la mine de Vipasca, voudra, au poids ou au volume, trier... préparer, broyer, cribler, laver des scories d'argent ou des scories de cuivre, ou de la poudre de scorie ou encore des concentrés de résidus de fonderie, ou celui qui entreprendra 
quis curauerit re/ece]rit|ue. Conductor omne genus calciamentorum praestare debeto: ni ita fecer[ $₫ £$, unicuique ubi uolet emendi] ius I esto.

(5) Tonstrini. ${ }^{1}$ Gonductor frui debeto ita, ne alius in $u$ [ico metalli Vipascensis inuel | territoris eius tonstrinum quaestus causa faciat. ${ }^{2}$ Qui ita tonstrinum fecerit, in sin/gulos ferramentorum usus $\mathrm{X}$. .] | conductori socio actoriue eius $\mathrm{d}$ (are) $\mathrm{d}$ (ebeto) et ea ferramenta commissa conductori sunto. ^[Excipiuntur serui] qui I dominos aut conseruos suos curauerint. ${ }^{4}$ Circitoribus, quos quos conductor [non miserit, tondendi ius ne es]to. ${ }^{5}$ Con|ductori socio actoriue eius pignoris captio esto. ${ }^{6} \mathrm{Qui}$ pignus capientem prohibuerit, [in singulas pro/ulbitiones $\mathrm{X} \mathrm{V} \mathrm{d(are)} \mathrm{|} \mathrm{debeto.} \mathrm{Con-}$ ductor unum pluresue artifices idoneos in portionem recipito. |

6) Tabernarum fulloniarum. Vestimenta rudia uel recurata nemini $\mathrm{m}$ [ercede polire nisi cui conductor so]|cius actorue eius locauerit permiseritue liceto. ${ }^{2}$ Qui conuictus fuerit aduersus ea qui[d fecisse, in singulas /ajcinias | XIII conductori socio actoriue eius $\mathrm{d}\left(\right.$ are) $\mathrm{d}\left(\right.$ ebeto). ${ }^{3}$ Pignus conductori socio actoriv[e eius capere liceto]. I

7) Scripturae scaurariorum et testariorum. ${ }^{1}$ Qui in finibus met[alii Vip ascensis... sctm]ri|as argentarias aerarias pulueremue ex scaureis rutramina ad mesuram pondu[>we purgare ............................ $\mathrm{re}$ expe|dire frangere cernere lauare uolet quiue lapicaedinis opus

ligne 2: fecer[¿£, unicuique ubi uolet emendi], au lieu de fecer[i£, circitoribus uendendi]

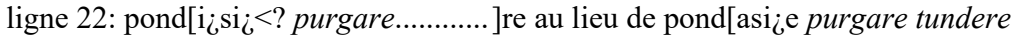
ure] re 
d'exécuter un travail dans des carrières de quelque façon que ce soit devra, dans les trois jours suivants, déclarer les esclaves et les salariés qu'il enverra exécuter ledit travail et devra payer... deniers au fermier chaque mois avant la veille des calendes; s'il ne le fait pas, il devra payer le double. ${ }^{2}$ Celui qui, d'autres endroits où ils abondent, amènera dans le territoire des mines des concentrés de résidus de fonderies cuprifères ou argentifères devra payer au fermier, à son associé ou à son agent un denier par cent livres. ${ }^{3}$ Pour toute somme qui, en vertu de ce chapitre de loi, sera due au fermier, à son associé ou à son agent,et qui, le jour où elle sera exigible, n'aura pas été acquittée ou cautionnée, il faudra payer le double. ${ }^{4}$ Le fermier, son associé ou son agent pourra prendre un gage, les scories qui auront été triées... préparées, broyées, criblées, lavées, (m Zuera les lauses qui auront été préparées dans les carrières, gw'iZ les saisisse si tout ce qui est dî à lui-même, à sow associé ou à son agent n'a pas été acquitté. ${ }^{5 N e}$ sont pas soumis à ces mesures les esclaves et les affranchis des fondeurs d'argent et des fondeurs de cuivre, gw SÉWÎ employés aux fonderies de leurs maîtres et de leurs patrons.

Chap. 8 - Les maîtres d'école

Il décidé que les maîtres d'école sont exempts de charge de la part du procurateur.

Chap. 9 - Prises de possession des puits ou impôt sur les écriteaux (ou droit de quittance)

Celui qui, à Vintèrieur du territoire de la mine de Vipasca, pour assurer son droit prendra possession d'un puits et du terrain de ce puits, ou bien qui les occupera conformément à la loi sur les mines, devra, dans les deux jours qui suivront la prise de possession ou Toccupation, faire connaître auprès du fermier de cet impôt, de son associé ou de son agent... 
quoquo modo facien[dum suscipiet, quos ad id] faciendum | seruos mercennariosque mittent, in triduo proximo profiteantur et soluan[i 3E... conductori quo]qlie mense | intra pr(idie) $\mathrm{k}($ alendas) quasque: ni ita fecerint, duplum $\mathrm{d}\left(\right.$ are) $\mathrm{d}\left(\right.$ ebento). ${ }^{2}$ Qui ex alis locis ubertumbis ae[raria argentariaue mjtramina in | fines metallorum inferet, in $\mathrm{p}$ (ondo) C X I conductori socio actoriue eius $\mathrm{d}($ are) $\mathrm{d}$ (ebeto.) ${ }^{3} \mathrm{Qu}[\mathrm{od}$ ex hoc capite] legis conduc|tori socio actoriue eius debebitur neque ea die, qua deberi coeptum erit, solu[tam satisue factum erit], d(uplum) d(are) d(ebeto). | ${ }^{4}$ Conductori socio actoriue eius pignus capere liceto et quod eius scauriae $\mathrm{pu}\left[\mathrm{r}^{\wedge} \mathrm{ta} . .\right.$.

expeditum frac] |tum cretum lauatumque erit quiue lapides

lausiae expeditae in lapicaedinis erunt commissa ei sunto, nisi quid]Iquid debitum erit conductori socio actoriue eius solutum erit; 5 ex[cipiuntur serui et liberti] | flatorum argentariorum aerariorum qui flaturis dominorum paironlorumque operam dant]. |

(8) Ludi magistri. Ludi magistros a pro(curatore) metallorum immunes es[se placet]. |

(9) Usurpationes puteorum siue pittaciarium. Qui intra fi [nes metalli Vipascensis puteum locum] |que putei iuris retinendi causa usurpabit occupabitue e lege metallis dicta, $\mathrm{b}$ [iduo proximo quod usurpauerit occupa] |uerit apud conductorem socium actoremue huiusce uectigalis profiteatu[r....................].

ligne 55 : ae[raria argentariaue $\mathbf{m}]$ tramina au lieu de ae[ris argentiue $\mathbf{r w}]$ tramina ligne 10/11: pu[rgatum..........expeditum frac] tum au lieu de pu[rgatum tunsum ustum expeditum frac $] \mathbf{t u m}$

ligne 17: es [se placet] au lieu de es [se concessum est] 
C. Le titre des chapitres

L'un d'eux est sûrement au nominatif. C'est celui du chapitre 9: Usurpationes puteorum siue pittaciarium. I] en va sans doute de même pour Je titre du chapitre 8, Ludi magistri, vu l'expression ludi magistros employée dans le chapitre même (25).

En revanche, le titre du chapitre 7, Tabernarum fulloniarum, est de toute évidence au génitif, ainsi que celui des chapitres 3 (Balinei fruendi), 4 (Sutrini) et 5 (Tonstrini).

Pour les autres - Centesimae argentariae stipulationis (chap. 1), Scripturae praeconii (chap. 2), Scripturae scaurariorum et testariorum (chap. 7) - il peut s'agir soit d'un nominatif pluriel, soit d'un génitif singulier.

En ce qui concerne Jes titres au génitif, on pourrait, à la rigueur, considérer qu'il s'agit de génitifs de relation («chapitre concernant...»), mais des génitifs ainsi isolés seraient surprenants, et il paraît préférable de les rattacher à un nominatif sous-entendu. Sans doute l'existence de titres dont les uns sont au génitif, les autres au nominatif est-elle le signe d'un manque de rigueur dans la présentation $\mathrm{du}$ règlement. Mais une telle latitude avait ses limites; en particulier, s'agissant des génitifs, il est peu plausible qu'on ait $\mathrm{pu}$ sous-entendre selon les cas des formules différentes, sous peine de confusion. Aussi admettrons-nous que pour les titres au génitif une seule et unique formule était à sous-entendre. Parmi celles qui ont été proposées (26) l'une, conductio, paraît à première vue particulièrement bien convenir ici, puisqu'il s'agit des conditions dans lesquelles sont mis à ferme des taxes ou le monopole de tel ou tel métier.

S'agissant des titres sûrement au génitif, conductio s'accorde avec ceux des chapitres 3 (Balinei fruendi), 4 (Sutrini) et 5 (Tons-

(25) Voir cependant la réserve de RE, p. 342.

(26) Pensio (Hübner, Lex metalli, p. 173), lex (WILmanns, p. 220). RE, p. 341, fait remarquer que locatio ne convient pas dans la mesure où les conditions énumérées par les divers chapitres concernent les conductores et non le locator, qui est ici le fiscus; pour la même raison, on écartera locatio conductio (J. FLACH, Table de bronze, p. 645-646). Mais cela suffit-il pour que l'on adopte conductio, à l'image de THIELMANN, p. 67 ? 
trini), mais pas avec celui du chapitre 6 (Tabernarum fulloniarum).

Les conditions qu'expose en effet ce dernier chapitre à propos des fouleries sont entièrement différentes de celles qui, au chapitre 3 par exemple, concernent le balineum. Dans ce dernier cas en effet c'est bien l'établissement de bains qui est loué, ou plus exactement sa jouissance. Qu'en est-il au chapitre 6? On pourrait d'abord penser qu'il traite de la location de fouleries appartenant au fisc, comme à Pompei, une foulerie, propriété de la colonie, était louée à un fermier ${ }^{(27)}$. Mais, s'il en était ainsi, le chapitre n'aurait-il pas un titre calqué sur celui du chapitre 3 - Tabernarum fulloniarum fruendarum par exemple — et surtout ne renfermerait-il pas des dispositions régissant les conditions d'exploitation des bâtiments et de ses installations? Or on n'y voit rien de tel; bien plus, l'existence de fouleries, propriétés de particuliers, semble impliquée par la première phrase du texte.

Considérons maintenant les titres de chapitres dont il est grammaticalement impossible de dire s'ils sont au génitif. S'agissant du chapitre 1 où, comme on le verra, la centesima en question désigne vraisemblablement l'impôt du centième sur les ventes, on pourrait sans doute considérer que centesimae est un nominatif pluriel, car un pluriel peut ici s'expliquer par le fait que, la centesima étant exigible sur chaque vente, le législateur considère la totalité des centesimae ainsi prélevées; mais à cela on objectera qu'il fallait aussi, à chaque fois, une nouvelle stipulatio , et pourtant, pour désigner cette dernière, c'est un singulier qui est employé. Aussi préférons-nous considérer que centesimae est un génitif singulier; si on le fait dépendre de conductio sous-entendu, le sens est parfaitement satisfaisant: le titre annoncerait clairement la mise à ferme d'un impôt, procédure tout à fait habituelle dans le monde romain.

On ne peut traiter séparément du titre du chapitre 2 et de celui du chapitre 7, car ils commencent tous deux par le même mot, scripturae, dont on peut penser qu'il a, dans les deux cas, le même sens et la même fonction. Dans le vocabulaire fiscal, scriptura a désigné «l'impôt sur les pâturages», et pourrait avoir

(25) CIL, IV, suppi., CXLI-CXLIV. 
conservé ici le sens général de «redevance» (28). Si Ton considère scripturae comme un génitif singulier dépendant de conductio sous-entendu, le titre du chapitre 7 («location de la redevance levée sur les scaurarii et les testarii») correspond très exactement au contenu de ce dernier, qui concerne la conductio de la taxe frappant, dans les cas mentionnés, scaurarii et testarii. En revanche dans les mêmes conditions, le sens est moins satisfaisant s'agissant du chapitre 2, dont le titre - scripturae praeconii - signifierait proprement «location de la redevance due à l'office de crieur public» $\left({ }^{29}\right)$, alors qu'à lire le chapitre 2 et en particulier ses premiers mots qui praeconium conduxerit — ce n'est pas une redevance qui est mise en location, mais bien l'office même de crieur public. Si bien que, si l'on retenait cette solution, ou ce serait introduire un nouveau personnage parfaitement inutile (le fermier de la redevance) à côté du fermier du praeconium, ou ce serait choisir une façon bien compliquée de désigner tout simplement ce dernier.

Considérer scripturae comme un nominatif pluriel permet, dans le cas des titres de chapitres 2 et 7 de faire l'économie du mot sous-entendu conductio. On pourrait alors justifier Je pluriel en faisant remarquer que chacun des chapitres traite non pas d'une, mais de plusieurs «redevances», dont le montant et le calcul varient d'après le volume de la vente ou la nature des marchandises dans le cas du chapitre 2, ou selon que les matériaux taxés viennent de l'extérieur ou sont d'origine locale (chapitre 7). Mais ces nominatifs pluriels sont plus intéressants encore si, avec d'Ors $\left({ }^{30}\right)$, on donne à scriptura de sens de «contrat écrit» que l'on trouve

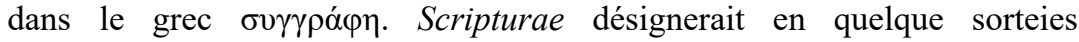
termes du contrat de location. Or, effectivement on connaît dans le droit romain récent la scriptura, qui accompagne souvent les contrats de vente, l'exécution de ces derniers étant subordonnée à la rédaction dudit écrit, qui renfermait les conditions du contrat $\left({ }^{31}\right)$. Scripturae pourrait avoir un sens très voisin, celui

(28) HÜ BNER, Lex metalli, p. 173; J. F LACH, Table de bronze, p. 659-660.

(29) Cette redevance étant considérée comme le «salaire» du praeco: cf. SCHONBAUER, Lex metalli, p. 182-183 et, plus récemment, ThielmanN, p. 61.

(30) D'ORs, Epigrafia, p. 88.

(31) GiRARD, Manuel, p. 570.

Conimbriga, 22 (1983), 5-193 
¿'《écrits renfermant des dispositions» qui concerneraient les domaines ou les personnes désignés par le ou les génitifs qui suivent: le praeconium d'une part, les scaurarii et les testarii d'autre part dans le cas des deux chapitres considérés.

Or on n'aura pas oublié les génitifs dont on s'inquiétait plus haut de savoir de quel mot sous-entendu on pourrait bien tous les faire dépendre. Scripturae «dispositions (écrites) relatives à...» répond bien mieux à cette exigence que conductio, dont on a vu qu'il ne convenait ni au titre du chapitre 2 , ni à celui du chapitre 6. En outre scripturae est écrit par deux fois en toutes lettres dans le texte de la loi. Par conséquent c'est ce mot que nous sous-entendrons devant les titres de chapities qui commencent par un génitif : celui des chapitres 3, 4, 5, 6 ainsi que, selon nous, du chapitre $1\left({ }^{(32}\right)$.

D. Commentaire

Io Chapitre I-(Dispositions écrites relatives à Vimpôt du) centième (sur le montant des ventes porté) sur la stipulation faite par le banquier

Le seul titre montre que le chapitre concerne un aspect de la vente aux enchères, $Y$ auctio, dans laquelle les ventes sont effectuées par l'intermédiaire d'un banquier-commissaire priseur (argentarius) avec l'aide d'un crieur public (praeco). Or la stipulatio argentaria est le contrat qui, dans une vente de ce type, est passé entre le banquier et l'adjudicataire (emptor), qui s'engageait ainsi à payer au premier le prix de la marchandise adjugée ( $\left.{ }^{33}\right)$. Cette stipulatio servait de base au calcul d'une centesima, une taxe dont la nature a été âprement discuté. Selon certains, il s'agirait là d'une rede-

(32) La diversité de structure que l'on remarque dans les titres peut s'expliquer par le fait que des chapitres ont été insérés dans le règlement à des époques différentes.

(33) Talamanca, p. 115-120; Thielmann, p. 71 et 132. Contra, Mommsen, Quittungstafeln, p. 102; Wilmanns, p. 220; D. Flach, Bergwerksordnung, p. 431. Sur la stipulatio en général, voir Gi Ra R D, Manuel, p. 511-519. 
vanee constituant en quelque sorte le salaire $\mathrm{du}$ banquier qui avait à Vipasca le monopole de la banque ${ }^{34}$, tout comme au chapitre 2 une autre centesima constituait le salaire (merces) du crieur public. Mais les deux chapitres diffèrent par leur contenu: alors que Vip. /, 2 précise les droits et les obligations du praeco, dont l'office est constitué en monopole, Vip. /, 1 s'occupe seulement des circonstances dans lesquelles la centesima est exigible, ce qui fait penser qu'il s'agit plutôt d'un impôt ( $\left.{ }^{35}\right)$.

Aussi d'autres voient-ils dans la centesima l'impôt de $1 \%$ sur les ventes aux enchères dont, au dire de Suétone ( ${ }^{36}$ ), Caligula avait exempté l'Italie en 39, mais qui a fort bien pu continuer à exister - ou ressurgir - dans les provinces, ce dont témoignerait Vip. /, $1{ }^{(37)}$. Cette hypothèse rendant compte des particularités du chapitre, nous l'adoptons de préférence à la première. Nous considérons donc que Vip. /, 1 concerne la taxe de $1 \%$ sur les ventes aux enchères. A Vipasca, cet impôt était mis à ferme; il était donc levé par un conductor, dans les conditions qui sont précisées par les différents articles du chapitre.

Le texte pose deux séries de problèmes, souvent liées d'ailleurs: des problèmes de restitution, dus aux lacunes de la table, des problèmes d'interprétation. La contribution de Thielmann à la solution de ces problèmes est capitale; c'est lui qui a poussé le plus loin l'analyse intrinsèque du texte, dans le cadre plus vaste d'une étude générale de Vauctio ( $\left.{ }^{3 S}\right)$.

$\left.{ }^{34}\right)$ Hübner, Lex metalli, p. 174 (= Hüвner, Metallum, p. 795); Mommsen, Quittungstafeln, p. 101; Bruns, Lex metalli, p. 379-380; J. Fi.ACH, Table de bronze, p. 651-654; Wilmanns, p. 220; Re, p. 343-348; HiRsch Feld, Ferwaltungsbeamten, p. 94; KNIEP, p. 14; D. FLACH, Bergwerksordnung, p. 430 .

(35) Thielmann, p. 68-69.

(36) Suet. Caligula, 1, 16.

(37) Demelius, Lex metalli, p. 33-35; Binder, Bergwerke, p. 230; Mispoulet, Tables d'Aljustrcl, p. 355; Schonbauer, Lex metalli, p. 356-362 et 381-383; d'Ors, Epigrafía, p. 82-83; Talamanca, p. 147-150; Thielmann, p. $67-70$ et $235-243$.

(38) Thielmann, p. 59-79. 


\section{a) Les restitutions}

1 -Pour le début de Vip. /, 1, 1, on a le choix entre deux restitutions: ea[rum stipulationum, quae ob auctio]nem $\left({ }^{3 \theta}\right)$ et ea[rum uenditionum, quae per auctio]nem $\left({ }^{40}\right)$.

Pour défendre la première restitution, on a tiré argument de la présence du mot stipulatio dans le titre du chapitre $\left({ }^{41}\right)$; mais il est au moins un chapitre de Vip. /, le chapitre 7, dans le texte duquel un des mots-clés du titre — testarii — n'apparaît pas. Ce n'est donc pas une règle générale que l'on doive retrouver dans le contenu des chapitres l'énoncé des notions mentionnées dan? le titre $\left({ }^{42}\right)$. Par ailleurs on a fait remarquer $\left({ }^{43}\right)$ que la restitution ...ea[rum stipulationum...] faisait naître deux difficultés: d'une part, à côté des stipulationes argentariae elle impliquait l'existence de stipulationes faites par le procurateur, dans le cas où il effectuait des ventes (...ea[rum stipulationumexceptis iis quas proc. metallorum... faciet); d'autre part, du même coup, la leçon stipulationum ne pouvait que désigner des stipulations faites par le vendeur et non plus par le banquier: alors, la centesima devait-elle être calculée d'après la somme figurant sur la stipulatio argentaria, comme l'indique le titre, ou d'après celle qui était portée sur la stipulation faite par le vendeur, ce qu'impliquerait la restitution

(39) Hübner, Lex metalli, ad p. 167 (= Hübner, Metallum, p. 791); Rodriguez de Berlanga, p. 629; ILS, 6891; Schonbauer, Lex metalli, p. 390; FIRA, I, p. 503; D'Ors, Epigrafía, p. 82-84; Talamanca, p. 118-119; D. FlaCH, Bergwerksordnung, p. 407.

(4o) Soromenho, fig. 3; estácio da Veiga, A tabula de bronze, fig. 5 et 6; J. Flach, Table de bronze, p. 655-656; Bruns, Fontes, p. 289; THIELMANN, p. 70-72 et 267 ; Girard-SENN, p. 84.

(41) Demelius, Lex metalli, p. 37.

(42) Thielmans, p. 70. La raison en est peut-être que les titres servaient simplement à attirer l'attention des usagers sur les points susceptibles de les intéresser, sans que l'on se fût préoccupé de la parfaite adéquation du titre au texte. Ainsi s'expliquerait encore la formule trop concise qui sert de titre à Vip. /, 1, mais centesima stipulationis argentariae mettait en évidence de façon pratique ce qui servait de base au calcul de la centesima.

(43) BRUNs, Lex metalli, p. 379-380; J. F LACH, Table de bronze, p. 655-656. 
adoptée? Il semble donc qu'en maintenant cette dernière, on ne puisse échapper à la contradiction. Thielmann y parvient cependant (44) en montrant d'abord que la seule stipulatio intervenant dans une auctio est la stipulatio argentaria, ensuite qu'en donnant à faciet une valeur factitive («fera faire»), on conserve à stipulatio son sens étroit, l'exception mentionnée concernant alors les stipulationes faites par le banquier à l'instigation $\mathrm{du}$ procurateur. Thielmann n'est cependant pas un partisan de la restitution stipulationum; on le voit bien lorsqu'il suppose qu'en vertu de sa position, le Procurateur pouvait organiser des ventes aux enchères sans avoir recours aux services d'un argentarius : du coup c'est la leçon uenditionum qui s'impose, d'autant que Vip. /, 2 renferme la formule ... ex pretio puteorum, quos proc. metallorum uendet.

L'argumentation de Thielmann n'est pourtant pas entièrement satisfaisante: d'une part en effet on ne connait, dans le monde romain, aucun exemple certain d'auctio qui se soit déroulée sans le concours d'un argentarius (45) et d'ailleurs le rôle joué par ce dernier dans la vente eux enchères était tel qu'on imagine mal qu'on ait $\mathrm{pu}$ se passer de lui, quand bien même le vendeur eût été le procurateur: il assumait d'abord l'organisation matérielle de la vente, ensuite, étant banquier en même temps que commissaire-priseur, il pouvait avancer à l'acheteur, en cas de besoin, l'argent nécessaire à l'achat, et c'était là une assurance pour le vendeur. On imagine mal le fisc jouant ce rôle vis-à-vis de l'acheteur : s'il a recours au système de la ferme, c'est en grande partie pour éviter cela. Il avait donc tout intérêt à faire appel dans tous les cas à Vargentarius ${ }^{(46)}$. Nous considérons par conséquent que, quand bien même c'est à l'instigation du procurateur qu'une auctio est organisée, un argentarius intervient et établit avec l'adjudicataire une stipulatio, la stipulatio argentaria dont parle le titre.

Est-ce à dire que nous admettions la leçon stipulationum en donnant à faciet une valeur factitive? En fait les solutions les plus

(44) THIELMANN, p. 71-72.

(45) Mommsen, Quittungslafeln, p. 94; Talamanca, p. 113; Andreau, Jucundus, p. 74 .

(46) Sur le caractère économique de l'intervention du banquier, voir TALAMANCA, p. 113 .

Conimbriga, 22 (1983), 5-193 
simples étant les meilleures, il nous paraît préférable d'adopter la restitution ea[rum uenditionum] qui a pour elle l'avantage de ne pas impliquer obligatoirement pour faciet une valeur factitive et l'appui de la formule qui apparaît dans Vip. 7, 1, 2, étant bien entendu cependant que le calcul de la centesima se faisait, comme l'indique le titre, en prenant pour base le prix atteint par les marchandises, tel qu'il figurait dans la stipulatio argentaria.

2 - Fin de Vip. 7, 1,1: la restitution [...a uendito]re accipito est quasi unanimement acceptée $\left({ }^{47}\right)$. Elle découle en effet de la comparaison avec Vip. 7, 1, 3 où est abordé le cas d'une vente aux enchères, qui, effectivement commencée, ne se serait pas terminée en tant que telle, si bien qu'on pourrait se demander si, dans ces circonstances, l'impôt est dû: or la loi dit que dans un tel cas le uenditor n'en doit pas moins (nihilo minus) la centesima au conductor. Ce nihilo minus doit renvoyer à un cas normal de vente aux enchères. Ce ne saurait être celui qui précède immédiatement, puisqu'il constitue précisément une des exceptions (exceptis iis quas proc. metallorum faciet) au cas général envisagé par Vip. 7, 1,1. En fait le point de référence ne peut être que ce cas général: il est donc normal que, dans l'une et l'autre situation, ce soit le même personnage qui supporte l'impôt, à savoir, comme l'indique la formule de Vip. 7, 1, 3, le uenditor.

3 - Fin de Vip. 7, 1, 6: Je cas particulier qui est envisagé ici - une vente aux enchères qui n'a pas abouti, les objets étant

(47) A l'exception de Schônbauer, Lex metalli, p. 385-386, suivi par D'Ors, Epigrafia, p. 83: [...a coacto] re accipito, une restitution qui ne s'impose pas ici. $U$ argentarius (ou argentarius coactor) est certes celui qui, dans une auctio, en tant que commissaire-priseur, reçoit l'argent de l'adjudicataire et peut en prélever la centesima rerum uenalium destinée au conductor de l'impôt (ainsi que le montant de son propre salaire, prévu sans doute dans un chapitre antérieur du règlement), avant de remettre au vendeur (dominus auctionis) la part qui lui revenait. Mais le législateur a eu ici le souci de mettre bien en évidence l'identité de celui qui avait à supporter effectivement l'impôt du centième, comme le montre à l'article suivant la formule... nihilo minus uenditor... praestare debeto. Sur l'identité du uenditor, voir THIELMANN, p. 76-78.

Conimbriga, 22 (1983), 5-193 
ensuite vendus selon une autre procédure, de condicione dit le texte - est parallèle à celui dont traitait Vip. I, 1, 3. C'est donc à bon droit que la totalité des éditeurs restitue un nihilo minus dans la deuxième lacune de l'article: ... de condicione uendiderit, nihilo minus con]ductori socio actoriue eius centesimam d(are) d(ebeto).

4- Fin de Vip. 1, 1, 2: deux restitutions ont été proposées pour la fin de cet article: Conductor ex pretio puteorum, quos proc. metallorum uendet ce[ntesimam ne exigito (48) ou ab emptore accipito $\left({ }^{49}\right)$ ]. Dans les deux cas on admet que le procurateur, en tant que uenditor de puits de mine, n'a pas à payer la centesima au fermier de cet impôt. Mais dans le premier cas \{ne exigito) on suppose simplement que cette vente ne produit pas d'impôt $\mathrm{du}$ tout, ce qui n'apporterait rien de nouveau par rapport à Vip. I, 1, 1, mais qui serait particulièrement étonnant s'agissant de puits de mine, la vente de ces derniers étant susceptible d'atteindre un prix élevé ${ }^{50}$ ) et par conséquent de rapporter au fisc un impôt supplémentaire important. D'autre part l'exemple de Vip. I, 2, 6 montre que, dans le cas considéré, la redevance au conductor du praeconium, qui était elle aussi égale au centième du prix de vente des puits de mine, était payée par l'acheteur. La deuxième leçon, ab emptore accipito, est donc préférable.

5 - Fin de Vip. I, 1, 4: elle a été complétée de deux façons différentes: Conductori socio actoriue eius, si uolet stipulari a

(48) HÜBNer, Lex metalli, ad p. 167 (= HübNeR, Metallum, p. 791); Mommsen, Quittungstafeln, p. 100; Re, p. 327; Demelius, Lex metalli, p. 48; ILS, 6891; FIRA, I, p. 503.

(49) J. Flach, table de bronze, p. 657; BRUNS, Lex metalli, p. 298; Rodriguez de Berlanga, p. 629; Binder, Bergwerke, p. 89; Schonbauer, Lex metali, p. 386; Talamanca, p. 148; Thielmann, p. 78-79; D. Flach, Bergwerksordnung, p. 407. A la place de accipito, J. FLACH, Table de bronze, Rodriguez de Berlanga, D'Ors, Epigrafia, p. 83, D. Flach, Bergwerksordnung, restituent exigito.

(50) Voir infra le commentaire de Vip. II, 2. 
ulenditore, is promittito... ( $\left.\left.{ }^{51}\right)\right]$ ou si uolet stipulari au[t pignus capere liceto $\left.\left({ }^{52}\right)\right]$. Stylistiquement et syntaxiquement, la première leçon laisse à désirer; en outre elle implique que seul le uenditor pourrait être débiteur du conductor de la centesima: or Vip. I, 1, 2 prévoit le cas où c'est l'adjudicataire qui doit payer cette dernière: on comprend mal dans ces conditions qu'il soit ici passé sous silence, et qu'il ne soit pas tenu, comme le uenditor, de passer une stipulatio avec le conductor si celui-ci Je désire (53). Aussi la formule plus générale qu'offre la deuxième leçon paraît-elle meilleure: elle s'inspire de Vip. /, 2, 8, et laisse au conductor de l'impôt, auquel on ne verse pas immédiatement la centesima dont on lui est redevable, la possibilité de s'assurer le paiement prochain de cette dernière, soit par une stipulatio au terme de laquelle le débiteur s'engage à payer sa dette dans un délai fixé ${ }^{(54)}$, soit en prenant un gage (pignoris capio), une procédure grave et qui ne peut s'expliquer ici que par l'importance de la somme que cet impôt pouvait parfois représenter $^{\wedge 5}$ ). Précisément, la loi laissait toute liberté de choix (si uolet) au conductor, et ce dernier devait sans doute se décider en fonction de l'importance de la somme à recouvrer (56).

(51) H Üв Ne R, Lex metalli, ad p. 167 (Mommsen, ibid., p. 175) = HüBNeR, Metallum, p. 795; Моммsеn, Quittungstafeln, p. 101 (is uenditor promittito); Bruns, Lex metalli, ad p. 374 (= Bruns, Fontes, p. 290); J. Flach, Table de bronze, p. 656-657; ROdRiguez de BerLanga, p. 629; FIRA, I, p. 503.

(52) Schonbauer, Lex metalli, p. 387-388; D'Ors, Epigrafía, p. 85; ThiELMANN, p. 65 et 267.

(53) Une stipulatio qui, en tout état de cause, n'avait rien à voir avec la stipulatio argentaria (cf. ThiеLmann, p. 239). Voir infra.

${ }^{(54)}$ La remarque de la note 53 vaut aussi pour cette interprétation.

(55) Sur la gravité de cette procédure, voir Talamanca, p. 150-151 et Thielmann, p. 65.

${ }^{56}$ Pour ce passage, une troisième restitution a été récement proposée par D. FLACH, Bergwerksordnung, p. 430-431 : conductori... eius, si uolet stipulari, aulctionem permittito']. Selon l'auteur, elle permettrait d'établir que la stipulatio argentaria était conclue entre le vendeur et Yargentarius. Mais une telle interprétation ne se conçoit et ne peut se discuter que dans l'hypothèse où l'on admet, comme le fait l'auteur, ibid., p. 429-430, que Vip. I, 1 concerne le monopole de la banque, donc que le conductor dont il est question dans ce chapitre n'est autre que le banquier. On sait que tel n'est pas notre point de vue. 


\section{b) Explication}

Compléter les autres lacunes du chapitre ne présente pas de difficulté particulière. Nous pouvons donc maintenant en venir au commentaire même du texte dont bien des aspects ont d'ailleurs été déjà abordés.

1 - A Vipasca, l'impôt du centième sur les ventes aux enchères était mis à ferme. Il était donc loué à un conductor, et c'est ce dernier qui recouvrait les sommes dues à ce titre. La rubrique placée en tête $\mathrm{du}$ chapitre fait connaître le document qui servait de base à rétablissement de cet impôt: il s'agit de la stipulatio argentaria, ou contrat de vente passé entre l'adjudicataire et le banquier-commissaire-priseur, et sur lequel était porté le prix atteint par les biens vendus. C'est le vendeur qui doit acquitter cet impôt, sauf dans les cas où le vendeur n'est autre que le procurateur agissant officiellement en tant que représentant du fisc (57). Le fisc en effet ne saurait être soumis à un impôt. Mais qui le payait alors? Nous reviendrons sur ce point à propos de l'article 2.

2 - L'article 2 aborde un de ces particuliers: il s'agit de la vente de puits de mines par le procurateur. L'impôt du centième est alors dû par l'adjudicataire.

On a déjà signalé la disposition parallèle que l'on trouve dans Vip. /, 2, d, et selon laquelle la redevance de $1 \%$ due au conductor praeconii est payée par l'adjudicataire, lorsqu'il s'agit pareillement de la vente de puits de mines par le fisc.

On mettra ces deux dispositions en relation avec Vip. //, i, 2 où sont indiquées les circonstances dans lesquelles le fisc procédait à la vente d'un puits en bloc (puteus uniuersus).

Le cas évoqué dans Vip. /, i, 2 nous amène à reconsidérer la question du paiement du centième dans le cas où Vauctio concerne des biens mis en vente par le procurateur. On remarquera d'abord que dans le chapitre 2 sur le praeconium existe une disposition

( ${ }^{87}$ ) D'où la formule iu/ssu imp (eratoris)], restitution due à BÜCHELER (Eph. Ep., 3, ad p. 167) et unanimement acceptée. 
(Vip. I, 2, 4) parallèle à celle où, dans Vip. I, 1, sont visés les cas qui échappent à la règlementation générale concernant l'impôt du centième sur les ventes aux enchères: si le procurateur met en vente ou en adjudication, en tant que représentant du fisc, des marchandises, le fermier de l'office de crieur public devra fournir un praeco pour la vente (ou la mise en adjudication), et cela, de toute évidence, gracieusement: autrement dit, dans tous ces cas - sauf bien entendu la vente de puits -, la redevance de $1 \%$ due au conductor de l'office ne lui sera pas versée.

Il n'y a pas tant de précision dans l'article 1. Pourtant la .formulation de Vip. I, 2, 4 montre à l'évidence que le procurateur était susceptible de mettre en vente des biens autres que des puits de mine. Qui payait alors l'impôt du centième ? En l'absence de toute indication, on peut conjecturer que la vente de ces biens était exonérée de la centesima rerum uenalium, et, vu ce qui se passe dans le cas de vente de puits de mine, c'était au bénéfice de l'acheteur. Le fisc pouvait ainsi vendre per auctionem certaines marchandises dans les mêmes conditions qu'un simple particulier; et s'il voulait attirer le public, il ne pouvait ni ne devait faire supporter aux acheteurs éventuels le montant de la

comme il le faisait pour les puits, car c'eût été les en écarter délibérément, puisqu'ils pouvaient les trouver ailleurs à meilleur marché, l'impôt qui en grevait la vente n'étant pas à leur charge mais à celle du vendeur. En revanche, la loi précise que, pour les puits vendus par le fisc, la centesima était due par l'acheteur. Cela ne devait pas avoir un effet trop dissuasif, car certains de ces puits au moins étaient riches $\left({ }^{5 S}\right)$ et, sans nul doute, très coiifoîtés. Comme ils devaient atteindre des prix passablement élevés, le montant de la centesima sur ces ventes compensait sans doute les pertes subies par le conductor de cet impôt en raison de l'exonération consentie par le fisc dans la vente d'autres marchandises $\left({ }^{59}\right)$.

${ }^{58}$ En particulier ceux qui étaient saisis dans le cas de fraude défini par Vip. II, 1. Voir infra le commentaire de cet article.

(59) Thielmann, p. 78-79. 
3 - Dans la critique qu'il fait des interprétations antérieures de ce passage, Thielmann $\left({ }^{60}\right)$ souligne en particulier la faiblesse de la position de Schonbauer ( $\left.{ }^{61}\right)$, selon qui Гauctio aurait été préparée, mais n'aurait pas eu lieu. Pourtant il est indéniable que cette dernière avait commencé (instituta auctione) et d'ailleurs s'il n'en avait pas été ainsi le verbe technique addicere («adjuger») n'aurait pas été employé. Il est donc possible de conjecturer, avec Thielmann, que, la vente aux enchères ayant commencé, une offre est faite pour l'ensemble du lot. Pour peu que le commissaire-priseur voie là une occasion de se défaire de marchandises qui autrement n'auraient pas trouvé preneur, il adjuge le tout en bloc \{universaliter omnia).

On pourrait objecter à cette procédure qu'il n'y a pas eu vente aux enchères véritable, chaque objet n'ayant pas été mis à prix ni adjugé au plus offrant. Néanmoins dans ce cas la centesima était due normalement par le vendeur.

4 - La restitution... a u[enditore is promittito], sans doute amenée par le contenu de la phrase précédente, avait plus ou moins consciemment fait considérer que les articles 3 et 4 étaient liés, au point que, même après la nouvelle restitution proposée par Schonbauer, ault pignus capere liceto], ce point de vue s'est maintenu ( $\left.{ }^{62}\right)$. Talamanca cependant a bien posé le problème $\left({ }^{63}\right)$ : pourquoi les garanties dont s'entoure le conductor seraient-elles prises exclusivement contre le vendeur, alors que l'acheteur aussi (article 2) peut être son débiteur? En fait la leçon de Schonbauer permet de considérer cet article-ci à part et de lui donner une portée générale: tous les débiteurs du fermier de la centesima étaient visés

(60) Thielmann, p. 73-75. Pour une interprétation analogue à celle de Thielmann, voir BRUns, Lex metalli, p. 381 et RE, p. 349.

(61) SChOnbauer, Lex metalli, p. 387.

(62) Schonbauer, Lex metalli, p. 387-388; D’Ors, Epigrafia, p. 86. Bruns passe cet article sous silence et seul HÜBNER, Lex metalli, p. 175, le considère à part, mais il y voit la preuve que la stipulatio argentaria était passée entre le vendeur et le banquier, et il estime qu'il aurait dû être placé ailleurs.

( ${ }^{63}$ ) Talamanca, p. 150-151, note 2. 
et si ce dernier le désirait, ils devaient soit passer une stipulation avec lui, soit lui fournir une caution. Cela n'était en rien contradictoire avec les dispositions de l'article 7 de ce même chapitre ( $\left.{ }^{64}\right)$.

5 - Le problème que pose l'article 5 est de savoir ce que désigne la summa quae excepta in auctione erit. On considère généralement qu'il s'agit d'une somme qui au cours de Vauctio, aura été déduite, pour une raison ou pour une autre, du total à payer à $Y$ argentarius. Cette disposition aurait eu une valeur générale et aurait visé dans la pratique un certain nombre de cas que la loi ne détaille pas. Divers savants ont tenté de définir quelques-uns de ces cas ( $\left.{ }^{65}\right)$, mais aucune de leurs hypothèses n'a fait l'unanimité.

La suggestion de Scialoja (66), qui attire l'attention sur le deuxième sens de excipere, «recevoir», n'a pas eu non plus grand succès. Elle n'est pourtant pas dénuée d'intérêt dans la mesure où elle nous fait nous interroger sur la possibilité de paiements au comptant au cours de $Y$ auctio même. Pour Talamanca cette possibilité existe juridiquement mais elle est seulement théorique $\left({ }^{67}\right)$, et il montre qu'en fait, même pour des sommes de peu d'importance, le paiement n'était pas immédiat mais était effectué par l'adjudicataire après stipulation. Pourtant si l'on donne à summa quae excepta in auctione erit le sens de «somme qui aura été reçue (par le banquier) au cours de la vente», c'est bien la possibilité de

(64) Pour la place de l'article 4 dans le chapitre, on se rappellera que l'absence de suite logique entre les divers articles d'un texte de loi romaine est fréquente (D’Ors, Epigrafia, p. 81).

(65) Par exemple la datio in solutum (le débiteur se libère en fournissant au créancier autre chose que la chose due), la compensatio (A étant créancier de $\mathrm{B}$ et devenant à son tour débiteur de ce dernier, les deux parties se mettent d'accord pour neutraliser les deux créances) ont été mentionnées par Bruns, Lex metalli, p. 381. Schonbauer, Lex metalli, p. 388, envisage une remise gracieuse. Mais, selon la remarque de Thielmann, p. 240, de tels arrangements étaient conclus après la vente et non, comme le veut la loi, in auctione.

(66) ScialoJa, Lex metalli, p. 482.

$\left({ }^{67}\right)$ TALAMANCA, p. 130-131: «le vendite a contanti erano possibili (sia in Roma che in Vipasca) ma soltanto su un piano astratto di possibilità giuridica» (p. 131). 
paiements au comptant qu'on envisage. Dans de tels cas en effet, aucune stipulation n'avait à intervenir entre le banquier et l'adjudicataire. Malgré cela, dit la loi, l'impôt du centième était dû. Dans ces conditions le paiement au comptant ne permettait pas d'échapper à l'impôt et l'on admettra que, grâce à l'inscription de la somme versée sur le livre de comptes du banquier, il était possible de le calculer. Une telle réglementation était dissuasive et devait engager les acheteurs à préférer la procédure de la stipulatio, qui par ailleurs permettait d'éviter les difficultés pratiques que, dans le cours d'une auctio, n'eût pas manqué de faire naître la généralisation abusive du paiement au comptant. Bref, cette interprétation nous paraît satisfaisante et finalement c'est elle que nous adopterons.

6 - Dans cet article, l'expression de condicione a un temps fait problème. Depuis J. Flach et Bruns, cependant, la majorité des commentateurs s'accordent à donner à cette expression le sens de «vendre de gré à gré»( $\left.{ }^{68}\right)$, qui correspond bien au sens premier de condicio, que l'on retrouve dans le verbe condicere «convenir de», «fixer en accord». Ainsi donc l'impôt est exigible par le conductor lorsqu'une vente aux enchères n'a pas produit l'adjudication escomptée par le vendeur mais que ce dernier a vendu ses marchandises de gré à gré, dans un délai de dix jours après l'échec de Vauctio.

7 - Le dernier article du chapitre concerne le délai de paiement de la centesima rerum uenalium au fermier de cet impôt. Ce délai est de trois jours. Passé ce délai, le montant de la somme due sera

(68) J. Flach, Table de bronze, p. 659; Bruns, Lex metalli, p. 382; Re, p. 351; Demelius, Lex metalli, p. 47; D'Ors, Epigrafía, 87; Thielmann, p. 242-243; D. FlaCh, Bergwerksordnung, p. 432. Cette interprétation est renforcée par l'opposition que signale Thielmann, p. 242, note 30, entre les deux verbes, addicere «adjuger» et uendere «vendre».

En revanche Mommsen, Quittungstafeln, p. 101, Hübner, Lex metalli, p. 175 et Wilmanns, p. 220, voient dans cette procédure une vente effectuée conformément aux conditions stipulées pour Vauctio entre le vendeur et le conductor (qui, pour eux, est le fermier de la banque). Enfin on notera a valeur factitive de addixerit. 
doublé. Les nuances de la terminologie utilisée par le législateur à propos du paiement des sommes dues sont difficiles à rendre en français. D’Ors détaille la spécificité des verbes employés ( $\left.{ }^{69}\right)$ : le débiteur pouvait payer purement et simplement (datum), se libérer autrement (mais c'est bien vague!) de sa dette (solutum) ou encore fournir une garantie (satisfactum). Parmi les garanties dont pouvait s'entourer le fermier, devaient figurer tant la stipulation que la pignoris capio, mentionnées l'une et l'autre à l'article 4.

Telles sont donc les prescriptions qui, à Vipasca, concernent la ferme de la centesima rerum uenalium. Elles s'appliquent exclusivement aux ventes aux enchères publiques, mis à part le cas de vente de gré à gré mentionné à l'article 6. Sans aller jusqu'à imaginer qu'il n'y avait à Vipasca qu'un seul conductor ou, comme l'avait d'abord pensé Hübner $\left({ }^{70}\right)$, qu'une seule société de fermiers qui aurait eu le monopole de tous les offices et de tous les métiers d'intérêt public, on peut bien admettre que plusieurs fermes étaient réunies entre les mains d'un seul conductor: ainsi il eût été commode que le fermier de la centesima eût été aussi celui de la banque, du moins dans la mesure où les acheteurs versaient sans difficulté à cette dernière le prix des marchandises $\left({ }^{71}\right)$.

\section{$2^{\circ}$ Chapitre 2 - Dispositions écrites relatives à Toffice de crieur public}

Dans ce chapitre, la restitution des lacunes pose moins de problèmes qu'au chapitre 1 . Il est cependant quelques cas qui méritent discussion.

Le premier est celui du début de la phrase 2, où la leçon de Hübner — [...Pro mercede ab eo qui uenditionem...] — a été géné-

(69) D'Ors, Epigrafia, p. 87.

(70) HüBNER, Lex metalli, p. 174. Bruns a immédiatement critiqué ce point de vue (BRUns, Lex metalli, p. 379-380) et HüBner, Metallum, p. 795 en a tenu compte.

(71) Ainsi l'application de la loi eût été singulièrement simplifiée, par exemple en ce qui concerne les articles 4 et 7 (cf. Thielmann, p. 239 et 243 . 
râlement acceptée (72). Seul J. Flach avait proposé une autre restitution - [Conductor, ab eo qui uenditionem] $\left({ }^{73}\right)$ - qui, quoique n'ayant pas reçu d'écho favorable, mérite qu'on s'y arrête. Non que le mot de merces soit ici inadéquat $\left({ }^{74}\right)$ mais les redevances mentionnées aux articles 3, 5 et 9 font elles aussi partie de la rémunération du fermier de l'office de crieur public, et pourtant le mot merces est absent de ces articles. Il ne s'impose donc pas davantage en tête de la phrase 2, où le mot conductor a tout autant sa place: nous l'y conserverons donc. Et l'on ne saurait nous objecter que cette mention était inutile, le sujet de la phrase 2 étant le même que celui de la phrase 1: que l'on se reporte à Vip. /, 2, où l'on verra que le mot conductor se trouve en tête de chacun des deux premiers articles.

Dans la deuxième lacune de la phrase 3, les éditeurs n'ont en général jamais tenté de rétablir le montant, en deniers, de la taxe que devra verser au fermier celui qui aura vendu moins de cinq esclaves. Récemment cependant, D. Flach, se référant à la phrase 2 de ce même chapitre où la taxe varie du simple au double selon que le montant d'une vente est inférieur ou supérieur à 50 (ou 100?) deniers, propose de fixer ce montant à six deniers (75). Mais cette restitution ne s'appuie que sur le seul exemple de la phrase 2 et la taxe a pu aussi bien se monter à 4 ou 5 deniers par tête qu'à 6. Aussi ne nous risquons-nous pas à préciser ce chiffre.

Enfin, au début de la phrase 5, Re a proposé libellum au lieu de inuentarium (76): l'un et l'autre se valent. Pourquoi donc introduire une variante? Nous conservons inuentarium.

(72) HÜBNER, Lex metalli, ad p. 167 et, pour les raisons qui fondent sa restitution, p. 175 .

(73) J. F LaCh, Table de bronze, p. 694, pi. IV.

(74) Cf. Schonbauer, Lex metalli, p. 182-183; Andreau, Jucundus, p. 81, note 1 , où il est précisé, à propos de la merces de 1' argentarius dont il est question dans plusieurs des tablettes de L. Caecilius Jucundus, que ce mot désigne «toute rémunération versée en cas de louage de choses corporelles ou non». La rémunération du praeco (ou du moins du fermier de ce monopole) ayant les mêmes caractères que celle de Yargentarius, il est donc normal de parler de la merces du praeco (cf. TALAMANCA, p. 142-143).

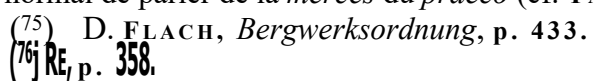

Conimbriga, 22 (1983), 5-193 
1 - Le premier article établit le monopole de l'office de crieur public. D'une façon générale celui qui prend à ferme cet office s'engage à fournir un praeco pour toute vente aux enchères publiques sur tout le territoire de la mine. On remarquera que le fermier de l'office n'exerce pas ]ui-même les fonctions de praeco, mais qu'il a des crieurs publics qu'il fournit à la demande $\left({ }^{77}\right)$.

2 - L'article 2 indique, en règle générale, le tarif de la redevance due au fermier par le vendeur. Ce tarif est à deux niveaux: il varie du simple au double $(1 \%$ et $2 \%)$, selon que la vente dépasse ou non cinquante (ou cent?) deniers $\left({ }^{78}\right)$.

3 - I] y a cependant des exceptions à la règle générale : on les trouve mentionnées aux articles 3,4 et 9 . Ici il s'agit des ventes d'esclaves; dans ce cas la redevance est calculée par tête et varie selon que le nombre d'esclaves vendus est ou non supérieur à cinq; dans le premier cas, la redevance est de trois deniers par tête. Dans le second cas, comme on l'a déjà vu, nous en ignorons le montant.

4 et 6 - Nous nous sommes déjà référé à ces articles au cours du commentaire de Vip. /, i, 1 et 2. La règle générale est que, sur les ventes ou les mises en adjudication faites par le procurateur en tant que représentant du fisc, le fermier ne touche aucune commission, bien qu'il soit tenu de fournir, gratuitement s'entend ( ${ }^{79}$ ), un praeco. Une exception cependant, et de taille: les ventes de puits par le procurateur, sur lesquelles il touchera $1 \%$, qui lui sera payé par l'acheteur.

5 - Cet article n'a guère intéressé les commentateurs de Vip. /, Re mis à part $\left({ }^{80}\right)$. D'après cet article, tout particulier, qui, ayant

(77) J. Flach, Table de bronze, p. 661-662.

${ }^{78}$ ) L'un des deux chiffres indiqués dans cet article est manifestement erroné, mais il n'y a pas de raison de choisir l'un plutôt que l'autre.

${ }^{(79)}$ En ce sens l'expression praeconem praestare (Vip. /, 2, 4) doit s'opposer à praeconem praebere (Vip. /, 2, 1).

(8o) RE, p. 358-359, dont le commentaire est discuté par TALAMANCA, p. 134 .

Conimbriga $_{i} 22$ (1983), 5-193 
des marchandises à vendre, le fait savoir par voie d'affiche est redevable d'un denier au fermier du praeconium. Re pense que la vente qui suivra est une vente privée, dans laquelle l'accord se fait directement entre acheteur et vendeur. Pour Talamanca en revanche, qui met cet article en relation avec le dernier $\left(\mathrm{n}^{\circ} 10\right)$ du chapitre, la vente ne peut être que publique, avec enchères et adjudication au plus offrant: l'affichage de Vinuentarium ne serait rien d'autre que l'annonce (proscriptio) d'une auctio. Mais le texte ne dit rien de tel; loin d'annoncer une vente dont le jour et l'heure seraient fixés, l'affiche a seulement pour but de faire savoir que $\mathrm{X} . .$. a des marchandises à vendre (cuiusque rei uendundae nomine), à charge pour les intéressés de s'entendre avec lui. Cependant, par cette publicité, X... empiète sur les prérogatives $\mathrm{du}$ crieur public, d'où la redevance fixée à un denier. Selon Re, elle est due pour chaque marchandise portée sur la liste: il a donné en effet à cuiusque la valeur distributive qui est d'ordinaire la sienne. Mais, dans cette hypothèse, la taxe serait lourde, au regard de toutes les autres qui sont mentionnées dans le chapitre, car elle ne frapperait pas la vente effective d'une marchandise donnée, mais la simple mention de cette dernière sur une affiche faisant connaître qu'elle est à vendre. Aussi préférons-nous donner à cuiusque un sens d'indétermination - celui d'omnis ou de quilibet - qu'il lui arrive d'avoir et considérer que la redevance d'un denier frappe seulement l'affichage de la liste.

7 - Cet article prévoit le doublement de la somme due au fermier du praeconium, si elle n'est pas payée dans les trois jours. $\mathrm{Au}$ chapitre 1, comme le montraient sa place (dernier article) et son début (Quod ex hoc capite legis conduction socio actoriue debebitur'])^ cette même disposition avait une valeur générale et concernait les diverses redevances dues au fermier de l'impôt du centième sur les ventes. Il doit en être de même ici. Sans doute la phrase 7 est-elle grammaticalement la suite logique de la phrase 6. Mais pourquoi la sanction qu'elle renferme viserait-elle seulement la taxe frappant la vente des puits de mine? Que l'on supprime la phrase 6 et l'on verra que la suite des idées est tout aussi logique avec l'article 5, et, l'article 4 mis à part, avec tous ceux qui précèdent, ainsi qu'avec l'article 9 qui suit. Il faut invoquer

Conimbriga, 22 (1983), 5-193 
ici, croyons-nous, cette absence d'ordre logique entre les matières qui, comme d'Ors l'a rappelé $\left({ }^{\delta 1}\right)$, caractérise le style juridique romain et qui est dû, selon toute vraisemblance, à la façon dont étaient compilées à l'intérieur d'un chapitre les dispositions successives le concernant $\left({ }^{82}\right)$.

8 - Comme l'article antérieur, celui-ci doit avoir une valeur générale et ce que nous venons de dire de la portée du précédent concerne aussi l'application de la pignoris capio, dont il traite.

9 - L'article 9 concerne la dernière des exceptions à la disposition générale qui, à l'article 2 , régit le montant de la redevance due au fermier du praeconium. S'agissant des bêtes de somme ou de trait, cette dernière est fixée à 3 deniers par animal, quel que soit le prix atteint par la vente.

10 - Le dernier article du chapitre renferme la même expression, de condicione uendere, que Vip. /, i, 6, au commentaire duquel nous renvoyons pour son explication. 11 s'agit de marchandises - esclaves ou tout autre objet — qui, au cours d'une vente aux enchères, n'ont pas trouvé preneur; il est cependant possible que, par la suite, dans une vente privée de gré à gré, leur propriétaire puisse s'en défaire. Si une telle vente avait lieu dans moins de 30 jours après que ces marchandises avaient été confiées au crieur public - entendons: après qu'elles avaient été offertes dans une auctio -, le vendeur devait acquitter au fermier la même redevance que si elles avaient été vendues aux enchères.

On n'a pas manqué de souligner les ressemblances entre les deux premiers chapitres de Vip. I $\left.{ }^{83}\right)$ - ils concernent le même

(81) D’ORs, Epigrafia, p. 81-82.

(82) Ainsi il serait plus logique que la phrase 5 ne soit pas intercalée entre la 4 et la 6 , et que les dispositions prévues par les articles 7 et 8 aient été placées à la fin du chapitre.

(83) J. Frach, Table de bronze, p. 600; Schonbauer, Lex metalli, p. 183; D’Ors, Epigrafia, p. 89. 
domaine: les ventes aux enchères publiques - mais aussi leurs différences ( $\left.{ }^{84}\right)$ : la ferme d'un impôt (chap. 1), n'est pas celle d'un office (chap. 2). Dans le premier cas, le fermier n'a pas d'obligation vis-à-vis du public; dans le second, il doit prêter ses services à ce dernier ou plutôt, dans le cas de Vipasca, disposer pour ce faire d'un personnel qualifié et le mettre à la disposition de la population.

Or que veut ici le législateur? Bien évidemment son premier souci est que la ferme des revenus du fisc rapporte à ce dernier le plus possible. Pour cela il faut intéresser les adjudicataires éventuels et faire en sorte que ces locations rapportent à leurs fermiers: tel est le but des articles qui énumèrent les diverses redevances et les garanties possibles assurant le recouvrement de ces dernières.

Mais il faut aussi, d'une ferme à l'autre, compenser les disparités: tel est le but, nous semble-t-il, au chapitre 2, du relèvement des tarifs de la redevance pour les petites ventes (articles 2 et 3) et du changement de base de l'impôt dans le cas de «marchandises» vivantes - esclaves, animaux de trait et de bât - où il est calculé par tête. Or main-d'oeuvre servile et force animale ( ${ }^{85}$ ) devaient être particulièrement recherchées pour le travail des mines. Elles alimentaient sans doute un marché actif, mais irrégulier en raison des disparités aux causes diverses (âge, conditions de travail, etc.) existant tant chez les esclaves que chez les animaux. L'établissement d'une taxe per capita viserait donc ici à assurer au fermier du praeconium un revenu plus régulier sur un marché qui ne l'était guère.

Les dispositions du chapitre 2 nous renseignent par ailleurs quelque peu, bien qu'indirectement, sur le nombre, le volume et le caractère des échanges à Vipasca. Si l'on accroît les taxes sur les petites ventes, c'est que celles-ci sont plus nombreuses que les autres, donc que parmi les acheteurs et les vendeurs ceux qui sont à la

(84) Thielmann, p. 68-69.

(85) Les animaux étaient utilisés même à l'intérieur des mines: on a découvert des squelettes de chevaux dans quelques mines antiques de la Péninsule Ibérique. 
tête de grosses affaires sont rares $\left({ }^{86}\right)$, constatation particulièrement intéressante pour qui veut se faire une idée de la situation des colons mentionnés par Vip. IL Pour ces derniers d'autre part, les problèmes de main d'oeuvre et de force animale sont capitaux: or, de même que l'absence d'un double tarif de redevance peut être, dans le cas de la force animale, le signe de la possibilité de transactions relativement importantes, sa présence, s'agissant des esclaves, semble indiquer que ceux-ci étaient achetés par petits lots plus que par lots importants, autre indice des faibles possibilités financières des entreprises minières de Vipasca et de leur petitesse.

\section{$3^{\circ}$ Chapitre $3-$ (Dispositions écrites relatives à) Texploitation de rétablissement de bains}

\section{a) Les restitutions}

Parmi les lacunes de ce chapitre, deux gênent plus spécialement les commentateurs: elles concernent le début des phrases 2 et 7 .

Phrase 2: La quasi totalité des éditeurs restituent: Aquam [in alueum usque ad] summam ranam... (87). Cette leçon paraît s'appuyer sur l'opposition que le passage de Vitruve sur les bains publics implique entre l'alueus et le labrum ( ${ }^{88}$ ) et qu'illustre, dans les thermes républicains de Pompei, la disposition du caldarium, aux extrémités duquel se trouvent respectivement Yalueus, ou baignoire d'eau chaude, et le labrum, ou vasque circulaire d'eau froide ${ }^{\left({ }^{89}\right)}$. Dans cette hypothèse, l'article traiterait de l'alimenta-

(86) Cf. Andreau, Jucundus, p. 95, note 1. Au vu des sommes portées sur les tablettes de Pompei, les ventes organisées par L. Caecilius Jucundus prennent l'allure de très grosses ventes par rapport à celles de Vipasca.

$\left.{ }^{87}\right)$ J. Flach, Table de bronze, p. 694, pi. IV; RE, p. 328; Bruns, Fontes, p. 291; Schonbauer, Lex metalli, p. 185; FIRA, I, p. 505; D'Ors, Epigrafia, p. 91; Thielmann, p. 269. La leçon balineum (Hübner, Lex metalli, ad p. 167; WILManNs, p. 222) est équivalente.

${ }^{(88)}$ Vitruve, De architectura, 5, $10,4$.

$\left.{ }^{89}\right)$ Thermes de Stables et du Forum: Crema, p. 72, fig. 78 et $\mathbf{7 9 ,}$ et p. 74. Le nom labrum est écrit en lettres de bronze sur la bordure du bassin des thermes du Forum: cf. LA Roca et alii, p. 135.

Conimbriga, 22 (1983), 5-193 
tion de Yalueus en eau chaude et du labrum en eau froide. Mais alors la difficulté réside dans la structure syntaxique de la phrase: en particulier quelle fonction attribuer à hypocaustis?

Sans doute pourrait-on restituer in alueis au lieu de in alueum. Dans ce cas hypocaustis se rattacherait naturellement à alueis. Mais alors il y aurait plusieurs baignoires d'eau chaude pour une seule vasque d'eau froide, et surtout pourquoi in alueis (ablatif) à côté de in labrum (accusatif)?

On est ainsi conduit à la restitution récemment proposée par D. Flach: Aquam [in aenis usque ad] summam ranam hypocaustis, et in labrum tam mulieribus quam uiris profluentem recte praestare debeto, où le groupe in aenis... hypocaustis n'est pas sans rappeler les aenea supra hypocausim tria componenda de Vitruve $\left(^{90}\right)$. Il s'agirait donc des chaudières en bronze destinées au chauffage de l'eau et groupées dans une chaufferie au voisinage du caldarium, comme dans les thermes de Pompei déjà mentionnés ou dans ceux, plus tardifs, de Lambèse ( $\left.{ }^{91}\right)$. Du coup la phrase est syntaxiquement plus correcte, l'accusatif in labrum s'expliquant par sa dépendance à l'égard d'aquam profluentem: en outre le chapitre ne concerne plus que la production et la fourniture d'eau chaude, mais, s'agissant de bains, n'est-ce point là la question essentielle ? Enfin labrum désigne une baignoire, au sens général du terme, et non une vasque pour l'eau froide. Le conductor devait donc veiller à ce que les chaudières fussent remplies d'eau jusqu'à un certain niveau ( ${ }^{92}$ ), usque ad summam ranam ( ${ }^{93}$ ) et à ce que, dans le labrum, cette eau chaude fût renouvelée deux fois par jour, une fois pour les femmes, une fois pour les hommes.

(90) Vitruve, De architectura, 5, 10, 1; D. Flach, Bergwerksordnung, p. $434-435$.

(91) KRETZChMer, p. 68.

(92) On a supposé que ce niveau était indiqué par une grenouille en bronze: dans la traduction proposée supra, on suppose qu'il n'y a qu'une rana; on pourrait aussi comprendre: «jusqu'à la grenouille la plus haute», ce qui impliquerait la présence de plusieurs grenouilles marquant des niveaux différents.

${ }^{(93)}$ Sur les diverses interprétations suscitées par cette expression, voir D’ORs. Epigrafía, p. 92.

Conimbriga, 22 (1983), 5-193 
Phrase 7; Ce qui subsiste de cet article implique qu'à la place de la lacune initiale, étaient évoquées les circonstances pouvant empêcher l'ouverture de l'établissement. De toutes les leçons qui ont été proposées, c'est celle de $\mathrm{Re}$ - [Si uis maior per aliquod tempus impediverit — qui nous paraît la plus satisfaisante, tant par son caractère suffisamment général que par la restitution du mot tempus, qu'implique forcément l'expression conservée eius temporis $\left({ }^{94}\right)$. Nous l'adoptons donc.

Enfin, à la phrase 5, la syllabe -ta du début de la ligne fera préférer la leçon [balineum et instrumenta ( ${ }^{95}$ ) plutôt que [instrumentum balinei et e]a, le plus souvent admis $\left({ }^{96}\right)$.

\section{b) Le commentaire}

1 - La première phrase définit la nature du service qui est demandé au fermier de l'établissement des bains. 11 doit assurer à ses frais le chauffage de ce dernier et l'ouvrir au public tous les jours aux heures indiquées, sauf décision contraire du procurateur: ainsi en effet peut s'expliquer la formule arbitratu procuratoris; par exemple la fermeture de l'établissement tous les trente jours pour nettoyage (art. 6) devait s'effectuer sous le contrôle du procurateur.

La durée du contrat est d'un an ; il se termine, suivant l'usage romain $\left({ }^{97}\right)$ à la veille des calendes de juillet, soit le 30 juin. On a fait remarquer aussi $\left({ }^{98}\right)$ la différence qui existe, pour les heures

(94) RE, p. 362-363. Autres formules: si qua necessaria refectio impedierit (J. Flach, Table de bronze, p. 694, pi. IV et p. 672; Schonbauer, Lex metalli, p. 186; F1RA, I, p. 505; D'Ors, Epigrafia, p. 93; Thielmann, p. 269; Girard-SenN, p. 593; D. Flach, Bergwerksordnung, p. 408), si non per conductorem factum erit (Mommsen, ap. Hübner, Lex metalli, ad p. 167), si per proc. metallorum tum factum erit (BÜCHELER, ibid.), si uis maior damnumue fatale impedierit (HIRSCHFELD, ibid.).

(95) J. Flach, Table de bronze, p. 694, pi. IV; RE, p. 328; D. Flach, Bergwerksordnung, p. 408.

(96) Hübner, Lex metalli, ad p. 167 ; Wilmanns, p. 222; Bruns, Fontes, p. 291; Schonbauer, Lex metalli, p. 186; Fl RA, I, p. 505; D’OrS, p. 92; Thielmann, p. 269; Girard-Senn, p. 593.

(97) J. FlaCH, Table de bronze, p. 664.

(98j ID., ibid., p. 665.

Conimbriga, 22 (1983), 5-193 
d'ouverture, entre Rome et Vipasca: à Rome les bains publics n'ouvraient qu'à la huitième ou à la neuvième heure, selon les saisons. A Vispasca, ils sont ouverts toute la journée, le matin aux femmes, l'après-midi aux hommes, une mesure qui s'explique sans doute moins par les conditions spéciales de travail dans un pays minier que par la petitesse de l'établissement, trop exigu pour abriter deux bains séparés, un pour les femmes, l'autre pour les hommes.

2 - La phrase 2 a été commentée lors de l'établissement du texte.

3 - La phrase 3 fixe le montant du droit d'entrée que les clients doivent payer au fermier: un semis pour les hommes, un as pour les femmes. Il est difficile de savoir exactement ce que représentaient ces sommes pour un ouvrier de Vipasca. A Rome, vers la fin du ler siècle, le bain coûte un quadrans ("), soit moitié moins que le prix payé par un homme à Vipasca. Sans doute manquons-nous de données qui nous permettent d'évaluer les ressources d'un ouvrier et le coût de la vie à Vipasca. On a cependant mis en parallèle les conditions de travail à Vipasca et à Alburnus Maior (Dacie), deux régions minières situées aux deux extrémités du monde romain, et on en a conclu à l'existence d'une situation à peu près comparable $\left(10^{\circ}\right)$. D'après un contrat de travail d'Alburnus Maior (tablette $\mathrm{n}^{\circ} 11$ ), le salaire journalier d'un mineur s'élevait à 2,3 sesterces par jour, et, d'après les prix pratiqués à Alburnus Maior pour certains produits alimentaires, on peut penser qu'un ouvrier dépensait quotidiennement pour se nourrir un sesterce et demi (101). Il disposait donc pour ses autres dépenses de 0,8 sesterces, soit plus de trois as par jour. S'il en était de même dans les mines de la Lusitanie méridionale, l'ouvrier de Vipasca pouvait aller chaque jour au bain public, mais on reconnaîtra que le droit d'entrée était élevé par rapport à celui qui avait cours à Rome.

H Martial, 3, 30, 4.

(100) MrozeK, Travail, p. 28-30.

(101) Mroze K, Mines cTor, p. 318.

Conimbriga, 22 (1983), 5-193 
4 - Un certain nombre de personnes avaient le droit d'entrer gratuitement: les affranchis et les esclaves impériaux en fonction dans les bureaux du procurateur ou allocataires du fisc. Bénéficiaient aussi de la gratuité les enfants et les soldats en garnison à Vipasca. C'était là autant de manque à gagner pour le fermier qui, par ailleurs, avait à faire face à de lourdes charges découlant des conditions d'exploitation.

5 - A l'expiration du bail, le fermier était tenu de rendre en bon état l'établissement de bains et ses installations, à l'exclusion de ce qui était usagé.

$\mathrm{g}$ - i] était donc capital d'entretenir régulièrement l'ensemble, mais plus spécialement les chaudières (aena), avec tout leur équipement, y compris en particulier les robinets qui y étaient adaptés et commandaient la distribution de l'eau chaude. Ainsi s'expliquerait la dernière mesure mentionnée ici (unguere adipe e recenti), car, s'il était évidemment nécessaire de laver et de gratter les chaudières, on comprend moins bien qu'il ait fallu les enduire de graisse fraîche: mais il n'en va pas de même pour la robinetterie. D. Flach pense que c'était pour combattre l'entartrage (102), mais l'eau d'Aljustrel n'est pas calcaire.

7 - Il s'agit d'abord de savoir ce que signifie... quo minus lauare recte possit. Cette expression renvoie-t-elle à la phrase précédente, comme le croit D. Flach (103) et comme pourrait le laisser penser le recours aux mêmes mots: recte lauare? En fait l'interprétation que, de Hübner à Thielmann (104), on a traditionnellement donnée de cet article est préférable. Il n'est pas douteux en effet que les dispositions qu'il renferme concernent les circonstances où le conductor a droit à une réduction du montant de la location, si, par cas de force majeure ou pour toute autre raison, il n'a pu, pendant une période donnée, jouir de la chose louée. Le Digeste renferme nombre de dispositions de ce

(102) D. FLACH, Bergwerkordnsung, p. 435.

(103) ID., ibid., p. 435-436.

(104) Hübner, Lex metalli, p. 177-178; Thielmann, p. 269. 
type et celle que citent à la fois Bruns et Re $\left({ }^{105}\right)$ est particulièrement éclairante: Aedilis in municipio balneas conduxerat, ut eo anno municipes gratis lauarentur; post tres menses incendio facto respondit posse agi cum balneatore ex conducto, ut pro portione temporis quo lauationem non praestitisset pecuniae contributio fieret (106). Il s'agit, dans les deux cas, de l'impossibilité où, les thermes étant fermés ou ne fonctionnant pas par cas de force majeure (incendie, dans le texte du Digeste), le fermier est dans l'impossibilité de prêter le service du bain (lauationem praestare), si bien que les clients ne peuvent se baigner (lauari, ou, dans Vip. I, 3, 7, lauare employé au sens réfléchi, comme il arrive parfois). Du coup le fermier subit un préjudice car, à Vipasca par exemple $\left({ }^{107}\right)$, il ne touche pas les droits d'entrée qui constituaient l'essentiel de sa recette: il déduira $\left({ }^{108}\right)$ de son fermage une somme calculée proportionnellement à la durée du préjudice.

8- Dans cette phrase, la formule si quid... fecerit est sur le même plan que haec, si bien que l'exception que marque praeter la concerne aussi. Gela signifie qu'en dehors des cas de force majeure visés par l'article précédent et en dehors des travaux qu'il aura effectués dans l'intérêt de l'exploitation des bains, le fermier n'aura pas le droit de déduire quoi que ce soit du fermage. Mais il doit être bien clair que les réparations et autres travaux rendus nécessaires par les conditions d'exploitation de l'établissement étaient à la charge du fisc( $\left({ }^{109}\right)$.

(105) Bruns. Lex metalli, p. 376; RE, p. 363.

(106) DIG., 19, 2, 30, 1.

$\left({ }^{107}\right)$ Dans le cas évoqué par le texte du Digeste, les conditions du contrat sont différentes, puisqu'il était prévu que les municipes entreraient gratuitement aux thermes, le prix d'entrée devant sans doute être réglé au fermier par la commune au vu du nombre d'entrées. Si l'établissement est fermé, il n'y a plus d'entrées; s'agissant d'un cas de force majeure, le fermier reçoit pourtant une compensation (contributio pecuniae) proportionnelle à la durée de la fermeture (pro portione temporis).

${ }^{(108)}$ Reputare a fréquemment ce sens dans les textes juridiques (J. FLACH, Table de bronze, p. 672; RE, p. 362).

$\left.{ }^{109}\right)$ Cf. D'Ors, Epigrafia, p. 92. Contra, J. FlaCh, Table de bronze, p. 673; Thielmann, p. 269; D. Flach, Bergwerksordnung, p. 411. A la place de praeter, Hübner, Lex metalli, p. 177, suivi par Wilmanns, p. 222-223, 
9-10-12 — Le bois est avec l'eau l'un des soucis majeurs des Méditerranéens. S'en procurer est un problème, d'autant que dans une région minière on en consommait beaucoup, tant pour le travail de la mine que pour les fonderies. Les dispositions contenues dans les phrases 9 et 10 traduisent de diverses manières cette pénurie. D'une part, l'approvisionnement en bois était une tâche si importante que, comme le fait remarquer Schonbauer (no), le fisc semble s'en être lui-même chargé, puisque c'est à lui que vont les amendes infligées pour manquement aux dispositions de la phrase 9. D'autre part sur le bois que recevait le fermier, sans doute par cette voie officielle, mais qu'il était tenu de payer (m), peut-être à prix réduit, il ne lui était permis de (re-)vendre que ce qui n'était pas bon pour le chauffage: nous traduisons ainsi l'expression ostili idonea non erunt, en pensant, à l'imitation de Hübner et de Bücheler (112), que - comme clauom pour clauum en 4, 2 - ostile est peut-être une graphie de * ustile (qui viendrait de urere), à savoir la pointe des branches qui avait été coupée (.recisamina, de recido, «retrancher», «rogner») (art. 9) de façon à ne conserver que le bon bois de chauffage. Parallèlement, le fermier était tenu d'avoir une réserve de bois suffisante pour plusieurs jours, sans doute en raison des difficultés toujours possibles d'approvisionnement (art. 12). Enfin le montant de l'amende en cas d'infraction aux dispositions interdisant la vente de bois était passablement élevé (art. 10): 100 sesterces représentent un mois et demi de salaire d'un mineur d'Alburnus Maior.

11 - Pour tout manquement à l'entretien de l'établissement de bains, le procurateur pourra infliger au conductor une amende d'un montant maximal de 200 sesterces.

La longueur du chapitre sur les bains publics trahit l'importance du rôle de cet établissement dans la vie des mines: il représentait

restitue propter, ce qui change totalement le sens de la prescription; mais l'un et l'autre mettent sur le même plan haec et si quid... fecerit.

(no) Schonbauer, Lex metalli, p. 187-188.

(m) Cf. Vip. I, 3, 1: conductor... omnia sua impensa balineum... calfacere... debeto.

(112) HüBNER, Lex metalli, p. 176-177 et BüCHELER, ibid. 
le confort minimum auquel l'administration impériale jugeait que les mineurs avaient droit. La rigueur des dispositions le prouve, ainsi que le taux élevé des amendes que le procurateur pouvait infliger au fermier en cas de mauvais entretien.

\section{$4^{\circ}$ Chapitre 4 (Dispositions écrites relatives au) métier de cordonnier}

Le chapitre 4 renferme les dispositions qui, par le biais de la location à un fermier, organisent le monopole de l'exercice d'un métier, ici du métier de cordonnier.

Celui qui prend à ferme l'exercice du métier de cordonnier en aura le monopole sur toute l'étendue de la mine de Vipasca. Toute concurrence est donc abolie dans les limites de ce territoire, où, seuls peut-être, des marchands ambulants (U3) sont susceptibles de faire quelque affaire, mais seulement au cas où le fermier ne pourrait pas répondre à la demande (art. 5).

Il nous semble que la restitution habituellement acceptée pour la lacune de l'article 1 - Qui calciamentorum quid lor amentor uтиe, quae sutores tractare so[lent, fecerit clauomue cali]garem fixerit... (114) réduit quelque peu la portée de ce dernier. Nous avions d'abord pensé que le texte original devait reprendre, à la forme voulue, le verbe tractare exprimé dans la relative qui précède, tout comme, à la ligne qui suit, uendidisse reprend uendere, et nous étions prêt à restituer tractauerit $\left({ }^{115}\right)$ à la place de fecerit. Tractare a en effet un sens plus large que facere, ce qui, nous semblait-il, correspondait davantage à l'esprit du texte. Mais les infractions à la série de dispositions prévues dans l'article 1 sont sanctionnées par une amende «du double»: il ne peut s'agir que du double d'une somme d'argent, représentant le prix reçu pour la vente d'un article. Aussi au lieu de fecerit, ou de tractanerit, restituerions-nous plus volontiers uendiderit.

(Пз) D’Ors, Epigrafia, p. 96.

(114) Hübner, Lex metalli, ad p. 167; Bruns, Fontes, p. 281; Flfí, I, p. 505; GIRARD-SENN, p. 594.

(m) Soromenho, fig. 2 hors texte. 
Ainsi donc le monopole de l'exercice du métier de cordonnier comprend principalement la vente de chaussures, de courroies, de clous pour les semelles, bref de tout ce qu'on est en droit de trouver chez un cordonnier. Il n'est pas jusqu au fait de ferrer une chaussure (art. 1) qui ne ressortisse à ce monopole, car cet acte suppose la vente préalable de clous (116). Au monopole des ventes, s'ajoute aussi celui de la réparation (art. 4), à l'exclusion $\mathrm{du}$ raccommodage des chaussures personnelles et, dans le cas d'un esclave, des chaussures de son maître. Les infractions au monopole des ventes sont punies d'amendes dont le fermier empoche le montant et qui équivalent au double des gains eflectués (ait. 1); il en allait de même dans le cas du fermier du centième sur les ventes $(1,7)$. Par ailleurs, ici aussi, la pignoris capio est autorisée (art. 3).

Une disposition est intéressante à deux titres. C'est celle qui oblige le fermier à pratiquer, pour la vente des clous de chaussures, les tarifs mentionnés dans une lex ferrariarum (art. 2). Elle montre en effet que le fermier n'était pas libre de fixer ses prix et que le fisc se souciait de protéger les habitants de Vipasca contre les excès qui eussent pu découler du monopole. Ensuite, elle se réfère à une lex ferrariarum dont nous ne savons par ailleurs rien d'autre. Les ferrariae sont les mines de fer. Mais s'agit-il d'une loi générale, valable sur toute l'étendue de l'Empire ${ }^{1}$ C'est possible, mais il peut aussi s'agir d'une loi de portée locale qui eût régi l'exploitation des minerais de fer contenus dans la montera (117) du gisement et qui aurait fixe le prix du fer et des objets de fer produits sur place: nous avons ailleurs (118) envisagé l'exploitation possible de minerais de fer dans les gîtes de pyrite du Sud-Ouest, dont fait précisément partie le gisement de Vipasca-Aljustrel.

(lie) D'Ors, p. 95. Sans doute le mot caliga désigne-t-il proprement la chaussure militaire. Mais il semble difficile de limiter à ce sens 1 expression clauis caligaris (contra, D. FLACH, Bergwerksordnung, p. 411) car la chaussure à clous est largement répandue dans le monde romain.

(117) Termes équivalents: «gossan» (angl.), «chapeau de fer» (fr.).

(iis) $\mathrm{y}_{0} \mathrm{j}_{\mathrm{r}}$ notre livre sur Les mines de la Péninsul Ibérique à Vépoque romaine (sous presse) et supra, p. 27-30. 


\section{$5^{\circ}$ Chapitre $5-($ Dispositions écrites relatives au) métier de barbier)}

A Vipasca, l'exercice de la profession de barbier n'était pas constitué en monopole absolu, dans la mesure où on pouvait pratiquer ce métier gratuitement (art. 1): tel est le cas (art. 3) des esclaves qui prêtaient leurs services à leurs maîtres (comme à propos des cordonniers) ou à leurs compagnons d'esclavage (119).

A ces exceptions près, le monopole concédé au fermier était entier dans la bourgade (uicus, d'après la restitution de la phrase 1) de Vipasca ainsi que sur les territoires qui lui appartenaient (art. 1). Même si la surface ainsi couverte n'était pas très considérable, les habitants étaient assez nombreux pour justifier l'existence de barbiers ambulants à la solde du fermier (art. 4). Il est d'ailleurs vraisemblable que c'est parmi ces circitores que le fermier recrutait les artisans compétents dont il devait s'assurer les services (art. 7) : les artifices idonei mentionnés dans la dernière phrase ne sauraient être en effet les socii avec qui il pouvait éventuellement partager la location et l'exercice du monopole, mais plutôt des ouvriers salariés que, pour faire face à une trop lourde tâche, il emploierait in portionem, chacun prenant sa part du travail.

Les mesures prévues à l'égard de ceux qui enfreindraient le monopole du conductor tonstrini sont sévères: une amende, dont nous ne connaissons malheureusement pas le montant, pour chaque infraction, et la saisie des instruments au profit du fermier (art. 2). En outre, il appartiendra à ce dernier ou à son représentant de recourir au besoin à Ia pignoris capio (art. 6) et, en cas d'opposition à l'exécution de cette dernière, d'infliger une amende de cinq deniers à son profit (art. 7).

(119) Sans doute, comme le pense Schonbauer, LCX metalli, p. 191, ces artisans pouvaient-ils recevoir une gratification, mais c'était dans le cadre de la domus ou de 1 'officina à laquelle ils appartenaient. Mais cela n'a rien à voir avec la merces que leur aurait valu l'exercice public de ce même métier. 


\section{$6^{\circ}$ Chapitre $6-($ Dispositions écrites relatives aux) fouleries}

On sait que, dans les fouleries (fullonicae, tabernae fulloniae), on apprêtait et on dégraissait les étoffes; le travail s'effectuait dans des cuves où l'on foulait aux pieds, dans un bain spécial, les étoffes et les vêtements à traiter. Cette activité n'était pas très propre, dégageait sans doute de mauvaises odeurs, et nécessitait des installations spéciales que les particuliers ne possédaient pas chez eux. Ils avaient donc îecours aux fullonicae $\left({ }^{120}\right)$.

A Vipasca, le droit de pratiquer le métier de foulon a été loué par un fermier (conductor). Cela ne signifie pas qu'il y ait eu des tabernae fulloniae en quelque sorte «municipales» comme il y avait un balineum «municipal» et qu'elles aient été prises à ferme dans des conditions analogues (bail d'un an; dispositions concernant l'entretien du matériel, les réparations, etc.) par ledit fermier. On peut même penser que, la loi n'en faisant point état, cette hypothèse est ici à rejeter. En revanche, si quelqu'un voulait ouvrir et exploiter pour en tirer profit (merces) une taberna fullonia, il devait en obtenir du fermier l'autorisation: ce dernier la lui accordait contre paiement d'une taxe (il s'agissait donc en fait d'une sous-location, d'où le verbe locauerit) ou gratuitement (tel paraît être le sens de permiserit) ; mais il pouvait aussi refuser. En tout cas, sans l'autorisation payante ou gratuite $\mathrm{du}$ fermier, il n'était pas possible d'ouvrir à Vipasca une boutique de foulon.

Les infractions à cette règle étaient fortement punies: trois deniers par vêtement foulé indûment (art. 2) ; par ailleurs, comme dans nombre de cas précédents, la pignoris capio était autorisée. Le montant élevé de l'amende montre que les contrevenants devaient être nombreux. C'est que les vêtements souffraient considérablement du travail de la mine et des fonderies. Il fallait donc fréquemment les changer ou du moins les réparer. Cela explique que les boutiques de foulons aient été particulièrement prospères à Vipasca, tant pour traiter les vêtements neufs (rudes) que les vêtements usagés mais rapiécés (recuratae). Aussi, avec la

$\left(! 2^{\circ}\right)$ Pour une description du travail et des fullonicae de Pompei, voir R. Etienne, La vi. quotidienne à Pompei, Paris, 1966, p. 163-166. 
perspective de ces gains substantiels, la tentation d'ouvrir une fullonica devait-elle être grande. Mais encore fallait-il, comme on l'a l'U, obtenir du fermier l'autorisation, sinon, en cas d'infraction constatée, l'amende pouvait être extrêmement lourde.

$\mathrm{Du}$ point de vue commercial, le régime que définit ce chapitre n'est pas celui du monopole, mais celui de la concurrence contrôlée. Il s'explique à la fois par la pression de la demande, par le souci de protéger les habitants de Vipasca contre des tarifs trop élevés, enfin, bien sûr, par le désir d'assurer au fisc des revenus importants et réguliers.

\section{$7^{\circ}$ Chapitre 7 - Dispositions écrites relatives aux exploitants de crassiers et aux carriers}

Tous les commentateurs ont souligné les problèmes que posent la compréhension et la traduction de ce chapitre. Plusieurs de ces problèmes sont suscités par des difficultés de vocabulaire. Aussi, avant toutes choses, doit-on s'efforcer sinon de résoudre ces dernières, du moins de les exposer. Nous le ferons en distinguant les deux types de matériaux dont l'exploitation fait l'objet de ce chapitre: les scories, et certaines catégories de pierres.

\section{a) Les scories}

Plusieurs commentateurs (121) ont douté que le mot scauria signifie «scorie» et lui ont donné le sens de «minerai». En fait les recherches entreprises ces dernières années dans les mines du Sud-Ouest montrent incontestablement que c'est bien de scories qu'il s'agit ici. Déjà G. Wilmanns pensait qu'avaient pu exister à Aljustrel des scories carthaginoises que les Romains auraient exploitées (122). Hübner et Bücheler font aussi venir le mot du

(121) Mommsen, dans Hübner, Lex metalli, p. 180; J. Flach, Table de bronze, p. 677-678; SCHONBAUER, Lex metalli, p. 209-210; D'Ors, Epigrafia, p. 101-102.

(122) Wilmanns, p. 227-228. 


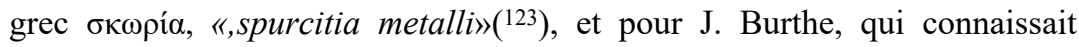
bien le site, l'exploitation de scories ne fait aucun doute $\left({ }^{124}\right)$. Mais c'est surtout J. M. Luzon qui, s'appuyant en particulier sur les travaux de L. U. SaUdeld, a assuré définitivement cette thèse $\left({ }^{125}\right)$.

La découverte de scories riches en argent dans des habitats d'époque tartessienne (ou phénicienne, comme on voudra) à Tharsis ( $\mathrm{H}$ 12) et à Riotinto $(\mathrm{H} 43)$ a montré que des crassiers relativement importants, datant de cette époque et constitués de scories de ce type, avaient dû exister dans ces mines (12e). Sans doute n'en avons-nous pas la preuve palpable pour Aljustrel, mais les conditions de gisement souvent analogues et la relative proximité font penser que plusieurs des gîtes pyriteux du Sud-Ouest ont dû être exploités alors pour l'argent $\left({ }^{\mathrm{m}}\right)$. Aljustrel est $\mathrm{du}$ nombre, et c'est aussi fort possible pour Herdade do Montinho (POR 11) et pour S. Domingos (POR 7), situés non loin de là.

Nous n'avons pas de témoignage certain sur l'exploitation du cuivre dans ces mines à l'époque tartessienne. Mais elles ont toutes ou presque toutes été travaillées à l'Age de Bronze par des mineurs qui étaient intéressés au premier chef par le cuivre. Parmi les scories cuivreuses qui, provenant de ces mines, ont été analysées, il n'en est aucune dont on puisse être certain qu'elle soit de l'Age $\mathrm{du}$ Bronze, mais nous connaissons des scories de cette époque - par exemple celles de Cerro Muriano (CO 39) - qui renferment 1 à $2 \%$ de cuivre: c'était là, s'agissant de scories, une teneur susceptible d'intéresser les exploitants romains.

On voit donc que des crassiers préromains renfermant les uns des scories argentifères, les autres des scories cuivreuses existaient

(m) HüBner, Lex metalli, p. 180 et Metallum, p. 801. De même Re, p. 376-378; BINDER, Bergwerke, p. 237; TÂCKHOLM, p. 125-126; THIELMANN, p. 271.

(124) Burthe, Tables de bronze, p. 37-39.

(125) Blanco Freijeiro-Luzón Nogué, Mineros antiguos, p. 76-77.

(126) Pour tout ce passage, voir notre livre sur Les mines de la Péninsule Ibérique à Vépoque romaine (sous presse), ainsi que SALKIELD, Ancient slags, p. 90-92. Il est également certain que des scories de ce genre remontent au moins au Bronze final.

(127) Voir supra, p. 27 et infra, Vip. Il, 14 à 18. 
très probablement à Aljustrel et clans les mines du voisinage au moment où les Romains exploitaient ces mines. Et si aujourd'hui on n'en trouve guère de traces - encore qu'à la fin du XIX ${ }^{\mathrm{e}}$ siècle $\left({ }^{128}\right)$, on nous parle des scories brunes, riches en cuivre, qui, remarquées à la base des crassiers dans les mines du Sud-Ouest, dataient peut-être de l'Age du Bronze — c'est peut-être parce que les haldes et scories d'époque romaine les ont recouverts, mais c'est aussi parce que, comme nous l'apprend ce chapitre, ils ont été exploités par les Romains eux-mêmes. Ce n'est d'ailleurs pas une nouveauté: ne savons-nous pas en effet qu'au Laurion à l'époque de Strabon on refondait des scories plus anciennes (129)?

Ainsi donc scauria désigne bien ici «la scorie» et n'est pas différent du mot scoria que l'on trouve dans Pline $\left({ }^{130}\right)$. Simple-

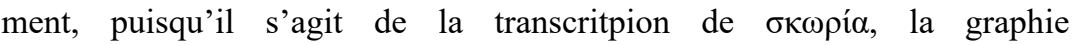
avec $a u$ doit vouloir rendre l'omega du mot grec.

Par ailleurs, scaur arias ne peut désigner qu'une personne s'occupant de scories. Nous traduisons par «exploitant de crassier», le crassier étant constitué par l'accumulation des scories ou crasses produites par les opérations de fusion. En Espagne, c'est l'«escorial», et, lorsque nous essayons de nous représenter ce qu'étaient ces scaurarii d'Aljustrel nous nous remémorons ces «cuadrillas» de quatre on cinq hommes, souvent des parents ou des gens du même village, que nous avons vues entre 1964 et 1970 dans la Sierra Morena et qui, dans les régions minières, complétaient leurs maigres ressources par des «extras» (on appelle ces gens les «sacagéneros») en allant laver les «escoriales» perdus dans la montagne et en vendant pour quelques milliers de pesetas à la fonderie voisine (La Cruz dans la région de Linares-La Carolina, Peñarroya plus à I Ouest) les concentrés obtenus. Ces «escoriales» sont les crassiers romains et ces gagne-petit les exploitaient naguère comme à l'époque romaine les scaurarii avaient exploité les scories tartessiennes et celles de l'Age du Bronze. Ces «cuadrillas» n'existent pratiquement plus aujourd'hui; des entreprises plus puissantes les ont

(128) Apud Salkield, Ancient slags, p. 90.

(129) Strabo, 9, 1, 23.

( ${ }^{130}$ j Plin., NH, 33, 69 et 105; 34, 107.

Conimbriga, 22 (1983), 5-193 
remplacées et lavent «escoriales» et haldes avec un matériel plus lourd et plus sophistiqué. Les «sacagéneros» avaient un équipement bien plus simple: d'abord des pics, des houes, des pelles, pour s'attaquer aux crassiers, desceller les scories, les dégager de la masse, les séparer des blocs de pierre et de la terre, pour mettre de côté celles que leur poids signalait comme étant riches en métal. Mais il y avait aussi les petits débris et surtout une terre noire que je les ai vus rechercher avec acharnement et qu'ils faisaient passer d'une main dans l'autre pour en apprécier le poids. Cette terre était celle qui avait constitué le niveau de travail de la fonderie, celle qui était la plus riche car les moindres débris ou les gouttes de métal qui étaient tombés sur le sol s'étaient mêlés à elle. Et cette terre, ils l'enrichissaient encore en la concentrant à l'aide d'un petit appareil peu encombrant, le «cajón», que les anciens traités de métallurgie appellent le jig à bras et qui, plongé dans un caisson d'eau, permet, par secousses successives, d'éliminer les parties légères et d'obtenir un excellent concentré. Ces «sacagéneros», nous les avons vus travailler exclusivement dans les escoriales de plomb, mais on peut sans peine imaginer des «cuadrillas» semblables, aussi simplement équipées et exploitant de façon comparable, à Vipasca, les crassiers préromains.

Les premières lignes de la phrase 1 nous apprennent ce que recherchent ces scauravii : scaurias argentarias pulueremue ex scaureis rutraminaue. A notre avis, les trois matériaux mentionnés ici proviennent tous des crassiers, y compris les rutramina. Ce dernier mot désignerait les concentrés obtenus par le lavage de cette terre noire présente dans toutes les fonderies mais ici, peut-être plus encore, des scories finement broyées. Ce sont ces concentrés que l'on remuerait et que l'on chargerait à la pelle (rutrum) (131).

En fait donc, le membre de phrase cité plus haut présenterait trois états différents du même matériau: d'abord les scories simplement triées et préparées, puis les scories broyées et réduites

(131) Hübner, Lex metalli, p. 181; Wilmanns, p. 228; D'Ors, Epigrafía, p. 102. Pour une interprétation un peu différente, voir BURThe, p. 39 (rutramina: «crasses d'affinage»). 
à l'état de poudre, enfin le concentré résultant du lavage de la poudre de scories. A ces trois états successifs correspondrait une série d'opérations exprimées par la suite de verbes purgare, [...], expedire, frangere, cernere, lauare. Les deux premiers concernent le simple triage (purgare: «purifier, nettoyer, débarrasser des impuretés, des matériaux gênants — sable, pierres, etc.» —, donc «trier») et la préparation (expedire) des scories à la main et au marteau: un troisième verbe concernait ce stade, mais il est difficile de le restituer: en tout cas, il ne saurait s'agir de urere, restitué d'après Pline (132) car il n'est ici aucunement question d'opérations métallurgiques, mais de simples procédés mécaniques. Frangere («broyer») et cernere («cribler») désigneraient le broyage des scories, puis le tamisage de la poudre ainsi produite, qui permet d'obtenir la granulométrie régulière qu'exige l'opération terminale. A cette dernière, le lavage, correspond lauare; à l'aide d'un appareil comparable à celui que nous évoquions plus haut, on éliminait les parties non métalliques, et même sans doute une partie du fer (densité: 7,8) de la scorie pour ne garder que les particules les plus riches en cuivre (densité: 8, 9) et en argent (densité: 10,5). Le résidu ainsi obtenu correspondait à l'ultime stade du travail des scaurarii, qui n'avaient plus qu'à aller le vendre aux fondeurs.

On comprendra ainsi que celui qui est allé exploiter d'anciennes scories hors du territoire de Vipasca dans ces lieux où elles abondent aussi (ex alis locis ubertumbis: ainsi traduit-on ce mot, par ailleurs inconnu, en le rapprochant de uber, «abondant»), sans doute dans une des mines voisines comme Juliana (POR 6) ou Caveira (POR 36), n'en ait ramené que Je concentré (rutramina), toutes les opérations, du triage au lavage, ayant été effectuées sur le lieu de travail. On comprendra aussi que dans la phrase 2 nous restituions aeraria argentariaue rutramina au lieu de aeris argentine rutramina $\left({ }^{133}\right)$, qui laisse croire que rutramina désigne des

(132) PLin., NH, 33, 69.

Í133) HüBner, Lex metalli, ad p. 167; Bruns, Fontes 3 p. 292; FI RA, I, p. 507 ; Thielmann, p. 271 ; Girard-SenN, p. 595 ; D. FlaCh, Bergwerksordnung, p. 409 (la façon dont cet auteur groupe les mots pour la traduction de ce passage ne nous paraît pas convenir au style du texte: voir ibid., p. 412 et 437). 
minerais. Enfin, vu la nature du matériau, on ne s'étonnera plus, à la différence de E. Schonbauer (134), du montant relativement élevé (un denier par cent livres) de la taxe qui frappait 1 entrée des rutramina à Vipasca.

\section{b) Les pierres}

La deuxième activité dont traite le chapitre 7 est l'exploitation de carrières de pierre (lapicaedinae) où l'on débite ( ) des lapides lausiae ${ }^{(135)}$. Les gens qui s'y consacrent sont de toute évidence les testarii, et c'est avec raison que l'on rapproche ce mot de testa que Pline emploie pour expliquer que la roche se fragmente en testae sous l'effet conjugué de l'humidité et du gel(136).

Mais que sont les lapides lausiae de l'article 4? Hübner a i appelé à propos de cette expression l'espagnol losa et le portugais lousa (137) ; Soromenho mentionne quant à lui la locution portugaise pedras de lousa, qui signifie «ardoise», «schiste ardoisier» $\left({ }^{138}\right)$. Par ailleurs on se souviendra qu'en France également, dans les Gévennes, la «lause» est un schiste grossier qu'on utdise pour couvrir les maisons. Effectivement le schiste est commun à Aljustrel, puisque il est la roche encaissante du gisement (139). Qu'il $\mathrm{y}$ ait eu des carrières où on le débitait en dalles on en plaquettes (lapideslausias expedire) est tout à fait vraisemblable. Et l'on peut alors penser, comme Hübner ou d'Ors $\left(1^{\circ}\right)$, que ces dalles étaient utilisées soit pour la confection de dallages soit pour le pavage des routes.

Une autre hypothèse est due à J. M. Luzon, selon qui il serait question, dans ce passage, de l'exploitation de roches aptes à seiAii

(134) Schonbauer, Lex metalli, p. 209.

(135) Lapis est en général du masculin, mais le mot est également attesté au féminin.

(i®·) Plin., NH, 33, 166.

(137) HÜв BER, Lex metalli, p. 181-182.

(138) Soromenho, p. 6. Voir aussi Rodriguez de BerLanga, p. 710.

(139) Freire de Andrade, Documentos inéditos, p. 78.

$\left(\mathrm{u}^{\circ}\right)$ HÜ BNe R, LCx metalli, p. 183; D’ORS, Epigrafía, p. 101. 
de fondants pour la fusion des minerais locaux (141). Les lapicaedinae mentionnés aux articles 1 et 4 auraient été destinées à l'abattage des «poches siliceuses» comprises dans la stratification des schistes $\mathrm{du}$ voisinage. La silice est en effet un fondant indispensable pour la métallurgie des minerais de cuivre contenus dans la pyrite de fer des gisements de cette région et dont la gangue est basique. La roche siliceuse par excellence est le quartz et au siècle dernier on ajoutait effectivement du quartz aux lits de fusion dans les fours de Riotinto (142). A l'époque romaine, dans la mesure où la gangue des minerais traités (chalcopyrite, chalcosite; oxydes et carbonates) était également basique (oxydes de fer, pyrite de fer), il était nécessaire d'utiliser du quartz, et comme on en trouve de nombreuses veines dans les roches encaissantes des gîtes pyriteux du Sud-Ouest, il était facile de s'en procurer.

Mais, au lieu de silex, qui est le mot habituel en latin pour désigner le quartz (143), on trouve dans notre chapitre 7 lapides lausiae. D'autre part, si l'on accepte le rapprochement entre lausiae et «lauses», «losas», «pedras de lousa», on conviendra que c'est bien le schiste qui est désigné ici, et non le quartz. Sans doute les schistes contiennent-ils une part de silice, mais elle est faible, si on les compare au quartz qui est de la silice pratiquement pure: pourquoi donc, s'il y avait du quartz dans la région, aurait-on utilisé le schiste comme fondant? On nous objectera que l'on trouve dans les crassiers de nombreux fragments de schiste: mais d'une part il y avait à Aljustrel des schistes cuivreux et d'autre part dans les crassiers foisonnent les débris de constructions diverses, en particulier de fours pour la confection desquels on a dû utiliser la roche commune du pays, le schiste. Pour notre part donc, nous renonçons à adopter l'hypothèse de J. M. Luzon: il ne faut pas vouloir tout interpréter par référence aux techniques minières et métallurgiques, surtout dans Vip. I. Nous considérons plutôt que les carrières dont il est ici question étaient destinées à fournir des matériaux de construction: les bancs de schistes y étaient

(141) Luzón, Instrumentos mineros, $\rho .233$.

(142) Pinedo Yara, Piritas, p. 618-619.

(143) Bailey, Chemical subjects, 2, ९. 270. 
débités en plaques et en plaquettes (lapides, lausiae) ( $\left.{ }^{144}\right)$, que l'on utilisait couramment dans les constructions, comme le montrent les vestiges d'édifices ensevelis ici et là sous les scories (fig. 9).

Mais pourquoi donc deux activités apparemment aussi diverses sont-elles abordées dans le même chapitre? 11 nous semble $\mathrm{d}$ abord qu'elles concernent deux matériaux qui se présentent de la même façon, en plein air, en surface, et que l'on exploite également par des découvertes. Mais surtout elles étaient soumises aux mêmes règles, comme nous allons le voir ci-dessous.

c)

\section{Les règles d'exploitation}

Ce qui est organisé dans ce chapitre, c'est le droit d'exploiter les crassiers et les carrières de pierre. Ce droit donne lieu au versement d'une redevance entre les mains d'un fermier. Par conséquent, quiconque veut exploiter des scories ou produire des pierres de construction doit d'une part le faire savoir au fermier dans les trois jours (il fournit pour cela la liste des ouvriers esclaves et salariés - qui seront employés à cette tâche), d'autre part lui payer un droit (art. 1). Comme il s'agit d'un travail de longue haleine, ce droit est dû chaque mois et doit être payé avant la fin du mois. Il devait être calculé selon le nombre d'ouvriers employés, puisque la liste de ces derniers est communiquée au fermier, et le tarif devait varier selon qu'il s'agissait d esclaves ou d'ouvriers libres. Il est probable que, dans le cas des scories et des rutramina, intervenait dans le calcul la quantité exploitée, qui se mesurait soit au poids, soit au volume (ad mesuram pondusue).

Une mesure spéciale concerne l'exploitation des scories (art. 2): c'est le droit qui est dû au fermier au cas où l'on ferait entrer sur le territoire des mines, pour être traités par une fonderie locale, des concentrés ( rutramina)de rebuts de fonderie venant d'ailleurs. Cette taxe est destinée à compenser le manque à gagner dont a été victime le fermier, puisqu'aucun droit d'exploitation ne lui a été versé, le crassier se trouvant hors des limites du

(144) Rien dans $\mathrm{i}_{\mathrm{e}}$ texte n'autorise à voir dans les lapides lausiae des «erzhaltige Steinsplilter» (D. F L A CH, Ber p. 436). 
territoire de Vipasca. On a vu plus haut pourquoi il n'était question ici ni de scories ni de poudre de scories. D'autre part l'entrée dans le territoire de Vipasca de lapides lausiae taillées hors de ce dernier n'est pas envisagée.

Les sanctions mentionnées en cas de non-paiement des droits à la date prévue sont diverses. Tout d'abord, il est précisé que toute somme due en vertu de ce chapitre de la loi doit être payée ou garantie le jour de l'échéance sous peine du double (art. 3). En cas de non-paiement, le fermier pouvait pratiquer la pignoris capio et se saisir des matériaux abattus et préparés: scories sous leurs différents états d'une part, plaquettes de schiste déjà débitées d'autre part (art. 4).

Une dernière disposition concerne les affranchis et les esclaves qui travaillent dans les fonderies de cuivre ou d'argent de leurs maîtres ou patrons: il est précisé qu'ils ne sont concernés ni par les taxes ni par les mesures de coercition mentionnées dans ce chapitre, puisqu'ils ne travaillent pas à leur compte (145). En particulier, il n'ont pas à souffrir des infractions commises par leurs maîtres et leurs patrons.

\section{${ }^{\circ}$ Chapitre 8 - Les maîtres d'école}

Il est exclu que les taxes dont seront exempts les maîtres d'école soient celles qui font l'objet des chapitres précédents et qui doivent être versées aux divers fermiers concernés. En effet, les maîtres d'école ne sont jamais nommés aux côtés de ceux qui n'y sont pas soumis. En fait, on considère généralement que l'immunité que la loi leur accorde ici concerne les munera civilia que pouvait imposer le procurateur en tant que responsable de l'organisation $\mathrm{du}$ bourg (146). Mais le procurateur est aussi le représentant du fisc, qui, par les dispositions énumérées dans les chapitres précé-

(U 5) Pour D. FLìch, Bergwerksordnung, p. 437, c'est, parce qu'ils n'ont pas appris le métier et qu'ils ne le pratiquement pas pour de l'argent.

(146) Hübner, Lex metalli, p. 185; J. Flach, Table de bronze, p. 685-687; D'Ors, Epigrafía, p. 103. 
dents, a loué à des fermiers l'exercice de diverses activités nécessaires à Ja vie du bourg et a reçu d'eux une redevance en échange. $\mathrm{Vu}$ ce contexte, ne peut-on penser que, par ce chapitre, les maîtres d'école étaient exempts de toute taxe portant sur l'exercice de leur métier et qu'ainsi ils occupaient à Vipasca une place à part? Autrement dit, l'éducation ne serait pas une activité dont l'exercice serait loué par le fisc à un fermier: cela signifie non pas qu'elle échapperait au contrôle du procurateur, mais que ceux qui exerçaient cette fonction ne devraient pas de taxe pour cela au fisc.

\section{$9^{\circ}$ Chapitre 9 - Prises de possession des puits ou impôt sur} les écriteaux (ou droit de quittance)

Ce chapitre traite d'une impôt appelé pittaciarium. Ce nom apparaît dans le titre sous la forme d'un nominatif neutre singulier, car l'hypothèse de Wilmanns( $\left.{ }^{147}\right)$ sur l'existence de putei *pittaciares qu'il faudrait admettre si l'on faisait de pittaciarium un génitif pluriel placé sur le même plan que puteorum n'est pas convaincante (148).

Etant donné la place de pittaciarium dans le titre, cet impôt se définit par une relation avec $Y$ usurpatio puteorum, mais il a aussi, vu son nom, un étroit rapport avec lat. pittacium / gr.

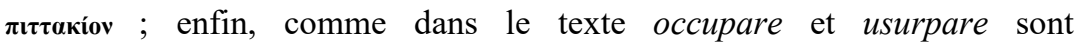
coordonnés par -we, la procédure désignée par le premier de ces verbes doit avoir aussi quelque lien avec le pittaciarium.

\section{a) Le «pittacium»}

Les commentateurs de ce chapitre se divisent sur le sens à donner ici à ce dernier mot: pour les uns, il s'agit d'un écriteau qui aurait été placé à l'entrée du puits de mine et qui aurait porté

(U 7) WILMANNS, p. 230.

(148) Hübner, Metallum, p. 800; DOrs, Epigrafia, p. 104; D. FlaCh, Bergwerksordnung, p. 438. 
le nom du possesseur $\left({ }^{149}\right)$; pour d'autres $\left({ }^{15 \circ}\right)$, le mot pittacium désignerait la quittance attestant le paiement de l'impôt correspondant à $Y$ usurpatio puteorum. Sans doute devait-on délivrer des quittances dans d'autres cas, mais on peut penser qu'il s'agissait là d'une quittance particulièrement importante, d'où le nom de pittaciarium qui se serait attaché à l'impôt en question.

Mais pittacium a pu aussi désigner un écriteau. Ainsi d'Ors rappelle que dans la langue latine pittacium est attesté au sens d'«étiquette»( $\left.{ }^{151}\right)$; il se réfère aussi d'une part à une tablette en plomb, trouvée dans la Sierra de Córdoba, mais aujourd'hui perdue, qui mentionnait l'occupation d'un lieu destiné à l'établissement d'un rucher $\left({ }^{152}\right)$, d'autre part aux petites étiquettes en bois trouvées dans les mines de Mazarrón et portant les noms de L. Minutius et de M. Minuf (...) (153). Mais ces deux derniers exemples n'ont pas grand chose à voir avec l'écriteau qui, portant vraisemblablement le nom de l'exploitant et placé à l'entrée d'un puits de mine, aurait eu un format bien plus grand.

\section{b) Usurpatio, occupatio}

Le mot usurpatio présent dans le titre est repris dans le texte par le verbe usurpare, mais ce dernier est alors couplé avec le verbe occupare: qui... usurpabit occupabitue. Le verbe occupare introduit-il donc une autre notion que celle exprimée par usurpare, et dans ce cas pourquoi cette notion n'apparaît-elle pas dans le titre du chapitre? Ou bien s'agit-il d'une seule et même notion, qui s'exprime par un doublet? Schonbauer (154) est du premier avis et distingue Yoccupatio (ou prise de possession) de Yusurpatio, qui serait le renouvellement — au bout d'une certaine période

(149) HÜBNER, Lex metalli, p. 186 (= HÜBNER, Metallum, p. 800); RosTOVTZEFF, Kolonat, p. 156.

$\left(\mathbf{1 5}^{\circ}\right)$ Schonbauer, Geschichte, p. 109-111, suivi par D. Flach, Bergwerksordnung, p. 438.

(181) Par exemple Satiricon, 34, 6 (cf. D’Ors, Epigrafia, p. 105).

(152) CIL, II, 2242.

(183) Goss É, Minas, p. 53 et pl. IV.

(154) Schonbauer, Geschichte, p. 111-113, suivi par Thielmann, p. 59, note 4. 
(il pense à cinq ans) — du droit d'exploiter qu'avait primitivement engendré 1'occupatio. Ainsi la formule juris retinendi causa porterait exclusivement sur le verbe usurpabit. Par ailleurs il faudrait conclure que le pittaciarium, vu le contenu même du titre, ne concerne que $V$ usurpatio. Mais pourquoi alors $Y$ occupatio est-elle mentionnée dans ce chapitre?

Pour d'Ors au contraire (155) les notions exprimées par occupare et usurpare sont très proches l'une de l'autre au point de se confondre. L'occupatio serait l'acte matériel de la prise de possession et $Y$ usurpatio en quelque sorte sa traduction sur le plan juridique, garantissant les droits acquis par l'occupation (l'expression juris retinendi causa porterait sur les deux verbes) et se manifestant par la pose d'un pittacium à l'entrée du puits.

Il nous semble cependant qu'une troisième explication est possible. Elle doit permettre en particulier de répondre à trois questions: pourquoi le mot occupatio n'apparaît-il pas dans le titre ? Pourquoi la fonction syntaxique de la formule juris retinendi causa donne-t-elle lieu à discussion? Pourquoi $Y$ occupatio et 1 usurpatio, ces deux notions si voisines, sont-elles mentionnées ici et juxtaposées par -ue? Nous remarquerons d'abord que cette loi de Vipasca ne constitue pas un bloc venu tout d'une pièce et d'une rigoureuse cohérence interne. En fait, au fur et à mesure de leur promulgation, les dispositions nouvelles étaient ajoutées dans le texte au chapitre concerné. De tels règlements sont donc en fait constitués de mises à jour successives. Plusieurs chapitres de Vip. I $\left({ }^{156}\right)$, le début et la fin de Vip. Il $\left({ }^{157}\right)$ paraissent bien avoir été construits de la sorte. Plutôt que d'«interpolations», il paraît préférable de parler d' «insertions» de mesures nouvelles.

Si l'on admet qu'il en est ainsi pour la mention de $Y$ occupatio dans Vip. /, 9, il est facile de répondre aux questions posées

(155) D’ORS, Epigrafía, p. 107-110. Récemment encore D. FLACH, Bergwerksordnung, p. 439, a exprimé 1 avis selon lequel les deux notions représentées par occupare et usurpare étaient très voisines l'une de 1 autre.

(156) Cf. les remarques de Schonbauer, Geschichte, p. 118-119, sur le manque d'ordre logique à l'intérieur des chapitres 2, 4, 5.

(157) $\mathrm{y}_{\mathrm{r}} \mathrm{i}_{\mathrm{r}}$ infra, p. 137 et $155-156$, à propos de la place des $\S 2$ et 15 qui coupent la suite des idées respectivement entre les $\S 1$ et 3,14 et 16 . 
ci-dessus. Le rédacteur de la mise à jour a jugé inutile ou a négligé de faire apparaître le mot occupatio dans Je titre; il a inséré la formule nouvelle occupabitue e lege metallis dicta immédiatement après usurpabit, de sorte que la formule juris retinendi causa porte effectivement sur les deux verbes; enfin Voccupatio désigne sans nul doute la façon nouvelle de revendiquer l'exploitation d'une concession (158): c'est sûrement une notion très proche de Vusurpatio (formule ancienne), mais le manque de données sur les modalités pratiques de l'une et de l'autre ne nous permet pas de les différencier. La mention des deux procédures unies par la copule -ue (usurpabit occupabitue) montre que cet état du règlement deVipasca établit un régime transitoire: Vip. II, qui ne mentionne pas 1 'usurpatio à côté de 1 "occupatio (cf. Vip. II, 1 à 5) doit marquer un état plus récent encore.

Toujours est-il que, dans le régime établi par Vip. I, 9, l'une ou l'autre des procédures aboutissait au même résultat. Par conséquente le pittaciarium était dû dans les deux cas. Ce serait donc un impôt frappant la «prise de possession» d'une concession minière (puteus locusque putei), qu'il ait été dû pour la délivrance d'une quittance attestant l'inscription de $Y$ usurpatio occupatioue sur un registre, ou à l'occasion de la pose d'un écriteau sur la concession elle-même. Cet impôt étant loué à un fermier, tout usurpator ou occupator devait payer à ce dernier soit un droit fixe, soit peut-être un droit calculé d'après la professio que, dans les deux jours, l'intéressé était tenu de faire auprès du fermier $\left({ }^{159}\right)$.

\section{c) Autres questions juridiques}

Trois points sont à préciser:

1 - Ce que désigne la locution «[puteus locusjque putei»

La première moitié a été restituée, mais cette restitution a reçu avec raison l'approbation de la totalité des commentateurs. A notre avis, cette locution n'est pas une simple redondance de

(158) Cf. Vip. II, 1 à 5.

(159) Voir supra, Vip. I, 7, 1. 
style juridique $\left.{ }^{(1 \beta 0}\right)$. Elle s'applique au puteus qui descend verticalement dans le sol et qui, avec son léseau de galeries et de boyaux, permet d'atteindre la minéralisation, mais qui ne saurait exister sans le terrain qui l'entoure et dans lequel il est (ou peut être) creusé. D'où la distinction entre puteus et locus, sans qu'on puisse séparer ces deux notions l'une de l'autre, sinon du point de vue juridique: alors que le locus putei désigne une réalité foncière (l'emplacement du puits), le puteus n'est qu'un instrument permettant l'exploitation des ressources souterraines du lieu. En outre il ne peut exister d'une part sans le sol dans lequel il s'enfonce et qui est propriété impériale, d'autre part sans le travail que consent l'exploitant pour le creuser. D'où les deux partes qui seront distinguées dans Vip. 1 et 2; l'une appartenant au fisc, l'autre à l'exploitant appelé occupator. En tout cas, ainsi unies, ces deux notions, puteus locusque putei, définissent une concession minière, à savoir une certaine superficie dont le périmètre permet également de déterminer en profondeur les limites (1 131$)$ à l'intérieur desquelles l'exploitation est susceptible de se développer: d'après Vip. II, 14 à 18 , on constate que la détermination des limites souterraines ne posait aucun problème.

L'emploi du mot puteus ne doit pas prêter à confusion: il ne signifie pas que, préalablement à toute usurpatio ou occupatio, le puits existe déjà, préparé par les soins du fisc, mais il définit le but et l'usage même de la concession. Dans cette expression puteus exprime plutôt l'idée de puits ou le puits en puissance. Le puits n'existe encore qu'en tant que possibilité et la concession ici définie est vraisemblablement une concession vierge. En effet, comme on le verra d'après Vip. II, la première phase de Yoccupatio (et il en allait sans doute de même avec 1 usurpatio) était précisément celle des travaux préparatoires, celles pendant laquelle on fonçait le puits jusqu'à la minéralisation. Les cas évoqués par Vip. II, 4 et 5 où il est fait expressément référence à Yoccupatio seront examinés infra, dans notre étude de Vip. II.

(160) Contra, Schon bauer, Geschichte, p. 114.

(161) Vip. //, 18 parle des fines putei. 


\section{2 - Le régime juridique d'exploitation défini par V«usurpatio» ou par V«occupatio»}

Habituellement, Voccupatio est considérée comme un mode originaire d'acquérir. C'est «l'acquisition d'une chose qui n'appartient à personne, résultant de la prise de possession de cette chose» (162). Or ici le fisc est propriétaire du sol et rien, ni dans Vip. I ni dans Vip. II, ne permet de penser que le fisc vende aux exploitants le terrain de la concession qu'ils mettent en valeur. Hoccupatio (et sans doute Vusurpatio) entraînait vraisemblablement une forme de possessio, ce que parait confirmer l'emploi du verbe usurpare, mis sur le même plan que occupare et qui signifie bien au sens juridique «prendre possession de»(163).

Il s'agissait donc d'entrer en possession d'une part d'un terrain concédé, le locus putei, ce qui implique la jouissance de ce dernier, donc le droit à l'exploiter et à tirer profit de sa richesse, ici le minerai; d'autre part d'un instrument d'exploitation, le puits, qui, comme on vient de le voir, appartient pour moitié au fisc.

Mais dans quelles conditions? Le contenu de Vip. II permet de définir le régime général de l'exploitation par occupatio. Nous en avons déjà mentionné quelques aspects, et, en anticipant sur l'étude détaillée de Vip. II, nous en résumons les traits principaux: d'un côté Xoccupator deviendra propriétaire (164) du puits à la fois par les travaux qu'il aura effectués pour le creuser (pars occupatoris) et, une fois la minéralisation atteinte, par l'achat de la part du fisc. De l'autre, il aura l'usufruit de la concession moyennant la remise de la moitié du minerai extrait au fisc: c'est une espèce de fermage à mi-fruit.

(162) Girard, Manuel, p. 330.

(163) Dig., 41, 3, 2, éd. Mommsen, Berlin, 1870.

(164) Vip. //, 2. 


\section{3 - L identification de la «lex metallis dicta» à laquelle renvoie ce chapitre pour la procédure de Voccupatio}

Cette lex metallis dicta n'est probablement pas celle dont le texte de Vip. I constitue lui-même une partie. Dans les chapitres précédents, lorsqu'une référence est faite à un chapitre de cette même loi Vip. /, on trouve l'expression ex hoc capite legis (Vip. /, i, 7). IJ n'est pas besoin en effet de préciser davantage: la lex sans autre détermination, c'est celle à laquelle appartient le chapitre en question ( $\left.{ }^{168}\right)$. Par conséquent si l'on nomme une lex metallis dicta, c'est qu'il s'agit d'une loi différente, tout comme la lex ferrariarum de Vip. I, 4, 2 a toutes chances d'être autre chose qu'un chapitre de Vip. I. Et, dans les deux cas, il s'agit de lois antérieures à Vip. $I$.

Par ailleurs nous avons vu que le texte de Vip. I est une veritable mosaïque juridique, faite d'un règlement originel et de mises à jour successives. L'une de ces mises à jour, apparemment récente puisqu'elle établit un régime transitoire, concerne Voccupatio et la référence qu'on y trouve à la lex metallis dicta montre que cette dernière doit être elle-même récente. Le fait que, dans cette locution, le mot metalla soit employé sans déteiminatif semble indiquer qu'il s'agit d'une loi générale: la résonance de ce pluriel indéterminé dans une telle locution nous paraît être différente de celle qu'éveille le mot metallorum dans Vip. /, 7, 2; qui... in fines metallorum inferet, où il est clair que le territoire concerné est celui des mines de Vipasca.

La lex metallis dicta dont il est ici question devait donc être une loi récente, qui exposait les principes généraux de la mise en valeur des mines impériales. Vip. /, 9 s'y réfère à propos de Voccupatio. Ainsi, entre autres règles d'exploitation, la lex metallis dicta semble avoir introduit à un certain moment dans les mines du fisc la procédure de Voccupatio.

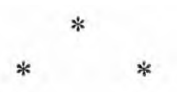

$\left.{ }^{168}\right)$ Cf. aussi dans les lois municipales de Malaca et de Salpensa: ex hac lege (Salp. 21, 22, 23, etc.; Mal., 51, etc.). 


\section{$10^{\circ}$ Conclusion}

On peut regretter que la première table d'Aljustrel nous ait fait connaître seulement une partie du règlement auquel elle appartenait, et que manquent en particulier la quasi-totalité des chapitres qui traitaient du régime d'exploitation de la mine, dont seules nous parlent les quelques lignes conservées de notre chapitre 9. Elle nous permet cependant de saisir au moins dans quel esprit l'administration impériale a alors organisé la vie de la mine, et en particulier comment la réglementation de toute l'activité qui régnait sur le territoire de la mine de Vipasca a pu créer un type spécial de communauté, vivant en dehors des structures municipales (on a parlé d'exterritorialité) habituelles dans les provinces.

\section{a) L'organisation de Vactivité}

A Vipasca, tout ce qui, dans l'activité quotidienne, était susceptible de rapporter quelque chose à l'Etat a été mis à ferme par l'administration impériale, du moins autant qu'on puisse en juger d'après les domaines abordés par les chapitres que nous connaissons. Ce qui était loué, c'était non seulement de simples taxes, comme l'impôt du centième sur les ventes (chap. 1) et le pittaciarium qui grevait la prise de possession d'une concession (chap. 9) ou encore des services publics tels que l'office de crieur public (chap. 2) et l'établissement des bains publics (chap. 3), mais c'était aussi le droit de pratiquer les métiers les plus communs - cordonnier (chap. 4), barbier (chap. 5), foulon (chap. 6), carrier (chap. 7) - auxquels il faut en ajouter un, plus original mais sans doute fréquemment exercé dans les régions minières, celui d'exploitant de crassiers (chap. 7).

Que par ce biais le fisc ait cherché à s'assurer des rentrées régulières et aussi substantielles que possible, c'est bien clair. Mais par là-même il réglementait étroitement, même à l'humble niveau de la vie quotidienne, la moindre activité ayant une incidence économique, et c'est à juste titre qu'on a pu parler à propos de cette loi de Vipasca d'un droit «contraignant» (le6). Et

(166) SCHONBÀUER, Ltx metalli, p. 211-214. 
effectivement toute personne qui, sur le territoire de Vipasca, voulait soit user d'un service, soit exercer une activité était contrainte d'entrer dans les cadres tracés par Tadministration impériale et surveillés, en quelque sorte, par les fermiers.

Cependant, si la loi crée des monopoles de fait dans certains domaines (crieur public, bains publics), d'autres sont moins absolus. Tout dépend en effet des conditions de la location: ainsi la possibilité qui est donnée aux habitants de Vipasca d'acheter ailleurs (entendons: hors du territoire de Vipasca ou auprès d'un marchant ambulant) si le fermier du sutrinum n'est pas à même de fournir la marchandise désirée $(4,5)$, desserre l'étau du monopole; en revanche, le fait que les esclaves qui coiffent leurs maîtres ou leurs compagnons $(5,3)$ ou, sans doute aussi, ceux qui réparent leurs propres chaussures ou celles de leurs maîtres $(4,4)$ sont dispensés de payer une taxe au fermier, n'atténue que bien peu les effets du monopole, puisque, dans les cas cités, il ne s'agit pas d'une activité rémunérée: au mieux elles peuvent faciliter un travail «au noir» sous l'apparence de la légalité.

Par ailleurs la loi se contente parfois d'établir ce qu'on pourrait appeler la concurrence contrôlée. C'est le cas au chapitre 6, où est prévue la sous-location du droit de foulage: ceux qui seront autorisés par le fermier contre versement d'une taxe à exercer ce métier auront le droit de le faire pour de l'argent $(6,1)$. Il est évident que si le fermier exerce convenablement ses fonctions, il autorisera ainsi l'ouverture d'autant de fullonicae qu'il sera nécessaire à Vipasca. Les dispositions relatives aux carriers et aux exploitants de scories sont du même ordre: il suffit d'une déclaration et du versement d'un droit au fermier pour pouvoir se mettre au travail $(7,1)$. Seulement la loi est peut-être ici moins rigoureuse dans la mesure où la seule véritable infraction définie concerne l'entrée de rutramina apportés d'ailleurs $(7,2)$.

$\mathrm{Au}$ fond la différence entre ces deux régimes - monopole et concurrence contrôlée — est facile à faire: si, pour l'exercice vénal d'une activité donnée, on tombe, quelles que soient les circonstances, sous le coup d'une amende, il s'agit d'un monopole. Si en revanche, il suffit de payer au fermier un droit pour y être autorisé, le régime est celui de la concurrence contrôlée. Il n'en 
reste pas moins que, dans Jes deux cas, on se trouve dans une économie dirigiste, excluant la libre concurrence.

Pourtant, si l'on considère les usagers, ce régime n'avait pas que des défauts. En effet on peut penser qu'aucun fermier n'était libre de pratiquer les prix qu'il désirait; c'est évident lorsque le montant du droit est fixé par la loi: centième sur les ventes $(1,1)$, droits dus au praeco selon la nature de la marchandise vendue $(2,1 ; 2,3 ; 2,4)$, entrée aux bains $(3,3)$. Dans un autre cas, il est fait référence aux tarifs prévus par la lex ferrariarum $(4,2)$. Il était donc possible aux agents du procurateur de surveiller les prix pratiqués par les fermiers; c'était l'intérêt à la fois des habitants de Vipasca et $\mathrm{du}$ fisc. Sans doute ces fermiers - qui n'on rien de commun avec ceux de la République car ils ne sont pas libres comme eux, ni même avec ceux des mines de Pannonie au II ${ }^{\mathrm{e}}$ siècle car leur sphère d'activité est plus réduite - gagnaient-ils quelque argent, mais, vu les dispositions prévues par la loi, ils ne pouvaient trop pressurer le bon peuple.

Dans le régime appliqué à Vipasca, nombreux étaient ceux qui, à des titres et à des degrés divers, pouvaient être satisfaits. Et d'abord le fisc, dont les rentrées étaient régulières et qui contrôlait l'activité économique et commerciale de tout le territoire de la mine. Ensuite les fermiers, à qui la levée des taxes, l'exercice d'un monopole ou d'un semi-monopole assuraient de bons revenus. Enfin, les habitants de Vipasca, dans la mesure où ils avaient recours aux services de ces gens, car soit les dispositions de la loi elle-même, soit l'existence d'une certaine concurrence devaient empêcher les prix de trop monter. Les seuls qui ont vraiment à se plaindre, ce sont les artisans, car en fait, la libre concurrence, dans la véritable acception du terme, n'existe pas à Vipasca. Par ailleurs, vu le système adopté, l'administration impériale ne prend en charge aucune forme d'activité: elle n'est donc pas «créatrice d'emplois». Sauf si c'est dans l'intérêt de l'Etat. Il est significatif que parmi les activités que mentionne Vip. /, il n'y en ait qu'une qu'elle se soit réservée: l'approvisionnement en bois (3, 10), car sans bois, ni l'activité minière, ni l'activité métallurgique n'étaient possibles. 


\section{b) La bourgade minière de Vipasca}

Ainsi organisée, la communauté de Vipasca se trouve hors de toute structure municipale. C'est ce caractère qu'on a défini sous le nom d'«exterritorialité» $\left({ }^{167}\right)$. Ce qui, dans une municipalité ordinaire, est du ressort de $Y$ ordo et des magistrats municipaux, revient ici à l'administration impériale et à celui qui par excellence la représente: le procurator metallorum. Dans la limite des dispositions prévues par la loi, tout dépend de $Y$ arbitratus procuratoris $(3,1)$. Ainsi alors qu'à Pompei, ce sont les magistrats municipaux qui s'occupent de la location de la fullonica appartenant à la colonie $\left({ }^{168}\right)$, à Vipasca c'est l'administration impériale qui met à ferme le bâtiment des bains publics (chap. 3). D’une façon générale, elle contrôle plus ou moins étroitement tous les secteurs d'activité de Vipasca mentionnés par la loi.

Ainsi décrit dans ses formes et dans son aboutissement, le régime qui règne dans le bourg minier de Vipasca et selon lequel y est organisée la vie d'une communauté humaine est clairement en marge du régime municipal romain.

Í167) BU R I A N, Exterritorialitàt, p. 49-52.

(168) CIL, IV, 3440, n ${ }^{\circ} 141$ à 144. 
(Página deixada propositadamente em branco) 
CHAPITRE II

VIPASCA II

La table de bronze renfermant le fragment de texte juridique connu sous le nom de «deuxième loi de Vipasca» (Vip. II) a été découverte le 7 mai 1906 à Aljustrel, «sous une couche de scories d'époque romaine» $\left({ }^{169}\right)$. Elle est conservée et exposée au Museu Nacional de Arqueologia e Etnologia de Belém (fig. 19). Ses mesures sont les suivantes: $770 \mathrm{mmx} 550 \mathrm{mmx1O} \mathrm{mm}$.

La table comporte, à gauche, une fracture qui a été réparée dans l'antiquité. De plus, la bordure gauche est irrégulièrement découpée et, comme de ce côté la marge est très étroite, des lettres manquent parfois au début des lignes, surtout lorsque le premier mot était décalé vers la gauche pour signaler le commencement d'un nouveau paiagraphe $(\S 10,13,15,16,17)$. A droite, la maige est beaucoup plus large. Des trous pour la fixation sont visibles en haut (2), sur le côté droit (2) et en bas (1).

L'adresse incomplète montre que cette table était précédée d'au moins une autre, et, comme le texte s'achève pai une phrase non terminée, il y en avait pour le moins une troisième, sans doute située au-dessous, car la largeur de la marge, à droite, suggèie qu'à ce niveau celle qui nous est conservée était la dernière.

La table est gravée d'un seul côté et compte 46 lignes. En ce qui concerne le texte lui-même, nous n'avons qu'une remarque à ajouter à celles qu'a faites $\mathrm{A}$. d'Ors $\left({ }^{170}\right)$ - comuictus au lieu de

(iew) Mïspoulet, Tables T Aljustrel, p. 346

Les mesures d'après CuQ, Règlement, p. 87.

$\left(17^{\circ}\right)$ D'Ors, Lex metalli, p. 127-129.

Conimbriga, 22 (1983), 5-193 
conuictus (1. 1) —et nous nous permettons de revenir sur trois des siennes: il semble bien qu'on lise intulerit et non intulebit (1. 8), Et ii(s\} colonies\} qui inpensam à la place de [V]el ii coloni qui inpensan (1. 20), apparuerit plutôt qu'abparuerit (1. 21). Par ailleurs, comme dans Vip. /, les points de séparation ont été placés de façon anarchique: on s'en convaincra en se reportant à la figure 19 . 
$\boldsymbol{A}$. Le texte et la traduction 


\section{La traduction}

[...] adresse son salut à son cher[.] Vlpianus Aelianus.

$\S 1 \quad[\ldots]^{1}$ Auguste, paiera comptant. ${ }^{2} S^{\prime}$ il ne le fait pas et s'il est convaincu d'avoir fondu du minerai avant d'avoir payé le prix comme cela est écrit ci-dessus, la part de l'occupant sera confisquée et le procurateur des mines vendra le puits tout entier. *Celui qui prouvera qu'un colon a fondu du minerai avant d'avoir payé le prix de la moitié (du puits) qui appartient au fisc recevra le quart (de cette somme).

$\S 2$ Les puits argentifères doivent être travaillés de la façon qui est contenu dans cette loi; leur prix sera conforme à la libéralité $d u$ très saint Empereur Hadrien Auguste, de telle sorte que la propriété de la part appartenant au fisc appartienne à celui qui, le premier, aura offert le prix pour le puits et aura versé au fisc quatre mille sesterces.

$\S 3{ }^{x}$ Celui qui, sur cinq puits, en aura poussé un jusqu'au minerai devra sans interruption travailler dans les autres comme cela est écrit ci-dessus. ${ }^{2}$ S'il ne le fait pas, un tiers aura le droit de les occuper.

$\S 4$ Si quelqu'un, après vingt-cinq jours consacrés à se procurer à l'avance l'argent nécessaire aux dépenses, a bien entrepris sans délai les travaux, mais s'est ensuite arrêté en cours d'ouvrage pendant dix jours consécutifs, un tiers aura le droit d'occuper le puits.

$\S 5$ Si un puits qui a été vendu par le fisc reste inactif pendant six mois consécutifs, un tiers aura le droit de l'occuper, dans des conditions telles que, lorsque du minerai sera extrait du puits, selon l'usage la moitié en soit garantie au fisc.

Conimbriga, 22 (1983), 5-193 


\section{Le texte}

] Ulpio Aeliano suo salutem,

(1) ...UVug. praesens numerato. ${ }^{2}$ Qui ita non fecerit et conuictus erit prius coxisse uenam quam pretium, sicut | supra scriptum est, soluisse pars occupatoris commissa esto et puteum uniuersum proc(urator) metallorum | uendito. ${ }^{3}$ Is, qui probauerit ante colonum uenam coxisse quam pretium partis dimidiae ad fiscum pertinendis numerasse, partem quartam accipito.

(2) Putei argentan ex forma exerceri debent quae | hac lege continetur; quorum pretia secundum liberalitatem sacratissimi imp(eratoris) Hadriani Aug. obser|uabuntur, ita ut ad eum pertineat proprietas partis, quae ad fiscum pertinebit, qui primus pretium puteo fecerit | et sestertia quattuor milia nummum fisco intulerit.

(3) ${ }^{\mathrm{J}}$ Qui ex numero puteorum quinque unum | ad uenam perduxerit, in ceteris ,sicut supra scribtum est, opus sine intermissione facito; ${ }^{2}$ ni ita fecerit, alii | occupandi potestas esto.

(4) Qui post dies XXV praeparationi impensarum dat $<$ a)s opus quidem | statim facere coeperit, diebus autem continuis decem postea in opere cessauerit, alii occupandi | [i] us esto.

(5) Puteum a fisco uenditum continuis sex mensibus intermissum alii occupandi ius. | [es]to, ita ut, cum uenae ex e o proferentur, ex more pars dimidia fisco salua sit.

ligne $3:$ su i supra \{aes)

ligne 17: datis (aes)

Conimbriga, 22 (1983), 5-193 
$\S 6$ 'Il sera permis à un occupant de puits de prendre les associés qu'ils voudra, dans des conditions telles que chacun contribue aux dépenses proportionnellement à sa part d'associé. ${ }^{2}$ Dans le cas contraire, celui qui aura assumé les dépenses fera afficher sur le forum, à Гendroit le plus fréquenté, pendant trois jours consécutifs, le compte des dépenses effectuées et signifiera à ses associés par le crieur public d'avoir à participer aux dépenses, chacun pour la part qui lui revient. ${ }^{3}$ Celui qui ne participera pas aux dépenses ou qui agira frauduleusement de manière à ne pas payer sa part ou à tromper un ou plusieurs de ses associés perdra sa part de puits et cette dernière appartiendra à celui ou à ceux de ses associés qui auront assumé les dépenses.

$\$ 7$ Et de plus, les colons qui auront effectué une dépense dans un puits où il y a plusieurs associés auront le droit de réclamer aux associés le paiement de ce qui manifestement a été dépensé de bonne foi.

$\S 8$ xIl sera permis aux colons de se vendre entre eux, aussi cher qu'ils pourront, même les parts de puits qu'ils auront achetées au fisc et dont ils auront payé le prix. ${ }^{2}$ Celui qui voudra vendre sa part ou en acheter une en fera la déclaration auprès du procurateur placé à la tête des mines; il ne sera pas permis d'acheter ou de vendre autrement. ${ }^{3}$ Celui qui sera débiteur du fisc ne pourra pas faire donation de sa part.

$\S 9$ 'Le minerai extrait et déposé près des puits devra être transporté aux usines par ceux auxquels il appartient, entre le lever et le coucher du soleil. '2Celui qui sera convaincu d'avoir enlevé $d u$ minerai d'auprès $d u$ puits après le coucher $d u$ soleil ou de nuit devra verser au fisc mille sesterces.

$\S 10$ xLe voleur de minerai, si c'est un esclave, le procurateur le fera fouetter et le vendra sous la condition qu'il soit enchaîné à perpétuité et qu'il ne séjourne dans aucune mine ni aucun territoire minier; le prix de l'esclave reviendra à son maître. ${ }^{2} \mathrm{Si}$ le voleur est un homme libre, le procurateur confisquera ses biens et lui interdira pour toujours le territoire des mines. 
(6) Occjul patori puteorum socios quos uolet habere liceto, ita ut, pro ea parte, qua quis socius erit, impensas | conferat. ${ }^{2}$ Qui ita non fecerit, tum is qui impensas fecerit rationem inpensarum factarum a se $\mid$ continuo triduo in foro frequentíssimo loco propositam habeto et per praeconem denuntiato $\mid$ sociis ut pro sua quisque portione inpensas conferat. ${ }^{3}$ Qui non ita contulerit, quiue quid dolo | malo fecerit quominus conferat, quoue quem quosue ex sociis fallat, is eius putei partem ne | habeto, eaque pars socii sociorumue qui inpensas fecerint esto. |

(7) Et $\mathrm{ii}<\mathrm{s}>$ coloni $<\mathrm{s}>$ qui inpensam fecerint in eo puteo, in quo plures socii fuerint, repetendi a sociis quod | bona fide erogatum esse abparuerit ius esto.

(8) dolonis inter se eas quoque partes puteorum, quas | a fisco emerint et pretium soluerint, uendere quanti quis potuerit liceto. 2Qui uendere suam partem | quiue emere uolet, aput proc(uratorem), qui metallis praeerit, professionem dato; aliter emere aut uendere | ne liceto. ${ }^{3} \mathrm{Ei}$ qui debitor fisci erit, donare partem suam | ne liceto.

(9) Wenas, quae ad puteos prolatae | [i]acebunt ab ortu solis in occasum, ii quorum erunt in officinas uehere debebunt; ${ }^{2}$ qui post occa|sum solis uel noctu uenas a puteis sustulisse conuictus erit, HS oo nummos fisco inferre debeto. |

(10) $x$ Venae furem, si seruos erit, procurator flagellis caedito et ea condicione uendito, ut in perpetuis | uinculis sit neue in ullis metallis territorisue metallorum moretur; pretium serui ad dominum | pertineto; 2 liberum procurator comfiscato et finibus metallorum in perpetuum prohibeto. | 
$\S 11$ Tous les puits seront soigneusement étayés et fortifiés, et le colon de chaque puits remplacera les boisages pourris par des boisages neufs et appropriés.

$\S 12$ II sera interdit de toucher ou de porter atteinte aux piliers ou aux supports laissés pour consolider la mine, ou encore d'entreprendre frauduleusement quoi que ce soit pour affaiblir ces piliers ou ces supports et empêcher ainsi le passage.

$\S 13$ Celui qui sera convaincu d'avoir endommagé ou détérioré un puits, d'avoir abîmé ses installations de surface ou d'avoir entrepris frauduleusement quoi que ce soit d'autre susceptible de nuire à la solidité $d u$ puits, si c'est un esclave, sera fouetté à la convenance du procurateur et vendu par son maître sous la condition qu'il ne séjourne dans aucune mine; si c'est un homme libre, le procurateur se saisira de ses biens au profit du fisc et lui interdira le territoire des mines à perpétuité.

$\S 14$ Celui qui foncera des puits cuprifères se tiendra éloigné du travers-banc qui évacue l'eau des mines et ne laissera pas moins de quinze pieds libres de chaque côté de ce dernier.

$\S 15$ II sera interdit de porter atteinte au travers-banc. ${ }^{2}$ Pour explorer le nouveau gîte, le procurateur permettra de pousser une galerie de reconnaissance à partir du travers-banc, à condition qu'elle ne mesure pas plus de quatre pieds en hauteur comme en largeur.

$\S 1611$ ne sera pas permis de rechercher ou d'abattre du minerai à moins de quinze pieds de part et d'autre du travers-banc.

$\S 17$ Celui qui sera convaincu d'avoir agi autrement dans les galeries de reconnaissance, si c'est un esclave, sera fouetté à la convenance $d u$ procurateur, et vendu par son maître, à condition qu'il ne séjourne dans aucune mine; si c'est un homme libre, le procurateur se saisira de ses biens au profit du fisc et lui interdira à perpétuité le territoire des mines. 
(11) Putei omnes diligenter fulti destinatique sunto, proque putri materia colonus cuiusque putei no|uam et idoneam subicito.

(12) Pilas aut fulturas firmamenti causa relictas attingere aut I uiolare doloue malo quid facere quominus eae pilae fulturaeue firmae et peruiae sint ne liceto. |

(13) Qui puteum uitiasse labefactasse decapitasse aliutue quid dolo malo fecisse quominus is puteus | firmus sit conuictus erit, si seruos erit, flagellis arbitratu proc(uratoris) caesus ea condicione a domi|no ueneat, ne in ullis metallis moretur; liberi bona proc(urator) in fiscum cogito et finibus ei metal|lorum in perpetuum interdicito.

(14) Qui puteos aerarios aget a cuniculo qui aquam metallis I subducet recedito, et non minus quam quinos denos pedes utroque latere relinquito.

(15) $1[\mathrm{Cn}] \mid$ niculum uiolare ne liceto. ${ }^{2}$ Proc(urator) explorandi noui metalli causa ternagum a cuniculo agere | permittito, ita ut ternagus non plures latitudinis et altitudinis quam quaternos pedes habeat. |

(16) [FJenam intra quinos denos pedes ex utroque latere a cuniculo quaerere caedereue ne liceto. |

(17) Qui aliter quit in ternagis fecisse conuictus erit, seruos flagellis arbitratu proc(uratoris) caesus ea condicione $\langle\alpha\rangle$ domino ueniet, ne in ullis metallis moretur; liberi bona proc(urator) in fiscum cogito et fini ¡ bus ei metallorum in perpetuum interdicito. 
$\S 18$ Celui qui foncera des puits argentifères se tiendra à distance du travers-banc qui évacue Veau des mines, et ne laissera pas moins de soixante pieds libres de chaque côté de ce dernier; dans les puits qu'il aura occupés ou qu'il aura reçus par assignation, il respectera, au cours de l'ouvrage, les limites telles qu'elles auront été fixées et ne les dépassera pas; il $n$ extraira pas de remblais minéralisés qui débordent de sa concession (ou bien il ne recueillera pas de haldes hors de ces limites), ni ne poussera de galeries de reconnaissance hors des limites d'un puits assigné... de façon à... 
(18) Qui puteos argentarios < aget ) a cuniculo, qui aquam metallis subducet, recedito et non minus quam sexagenos pedes utroque latere $i$ elixi] quito, et eos puteos quos occupauerit adsignatosue acceperit in opere uti determinati erunt habeto nec ultra procedito neue egbolas colligito neue ternagos ita agito extra fines putei adsignati, [...ut...] 


\section{B. La division du texte}

Nous avons plus haut pailé de la division en paragraphes. Il nous semble que, vu le contenu de ces derniers, le sommaire de la partie du texte qui nous a été conservée peut être présenté ainsi:

$\S 1$ à 10: Régime juridique d'exploitation des puits

[.......paragraphes antérieurs inconnus...................................... ]

1 - Droit d'exploitation des puits par occupatio.

2 - Dispositions spéciales concernant Voccupatio des puits argentifères.

3 à 5 - Conditions d'exploitation des puits par occupatio. Caducité des droits.

6 à 7 - Formation et fonctionnement des sociétés destinées à faciliter le financement des opérations minières.

8 - Cession des parts de puits.

9 et 10 - Conditions du traitement des minerais. Dissimulation et vol.

$\S 11$ à 18: Mesures techniques concernant la sécurité dans les mines

11 à 13 - Etayage et fortification des puits et des mines.

14 à 18 - Précautions à observer à l'égard du traversbanc d'exhaure.

14 - Distance minimale entre le travers-banc et les puits cuprifères.

15 - Usage du travers-banc pour l'exploration du nouveau gîte: la galerie de reconnaissance.

16 et 17 - Règles à observer dans la recherche et l'abattage du minerai dans les puits cuprifères, de part et d'autre du travers-banc. Sanctions en cas d'infraction.

18 - Distance minimale entre le travers-banc et les puits argentifères. 


\section{Commentaire}

Les dispositions dont on vient de lire le texte se divisent en deux groupes: les dix premiers paragraphes sont des règles à caractère juridique et fiscal, les huit derniers renferment des mesures d'ordre technique. Le commentaire que nous en donnons ci-dessou; suit cette division.

\section{$1^{\circ}$ Le régime juridique d'exploitation des mines}

\section{a) $\S 1$ et 2 : Droit d'exploitation des puits par «occupatio». L'wccupatio» des puits argentifères}

Il est difficile de mener séparément l'étude de chacun des deux premiers paragraphes. Nous les considérons donc ensemble.

Le paragraphe 1 est incomplet. Si l'on compare ce qui en reste avec le paragraphe suivant dont les dispositions semblent constituer une exception touchant les puits argentifères, il est vraisemblable que le paragraphe 1 concernait le régime général d'acquisition des puits et d'exploitation des concessions minières de Vipasca selon la procédure de Yoccupatio.

Le mot occupator nous rappelle Vip. I, 9, où, à côté de $Y$ usurpatio, était mentionnée Yoccupatio d'un puteus locusque putei. Pour toutes ces notions, nous renvoyons au commentaire que nous avons donné ci-dessus de ce chapitre.

Par la procédure de Yoccupatio, un exploitant prend donc, à son initiative, possession d'une concession minière. Il est d'abord autoiisé à entreprendre dans cette dernière des travaux préparatoires (creusement d'un puits) en vue d'atteindre le minerai. Gela constitue la première phase de l'exploitation comme le montre Vip. II, 3, où il est prévu que, lorsque sur cinq puits l'un aura atteint la minéralisation, l'exploitant devra immédiatement entreprendre des travaux dans les quatre autres, faute de quoi il perdra ses dioits et un autre exploitant pourra occuper ces concessions. Pour quoi faire? sinon justement pour exécuter ces tra ${ }^{\mathrm{r}}$ aux préliminaires.

Comme dans le paragraphe 1 on parle de minerai à fondre, il apparaît que cette première phase est achevée. Mais, avant de 
commencer à fondre le minerai, l'exploitant doit payer un certain prix (pretium), dans $\mathrm{d}^{\wedge} \mathrm{s}$ conditions qui étaient signalées plus haut \{sicut supra scriptum est) et dont, dans le meilleur des cas, il ne reste que trois mots: [...] Aug. praesens numerato.

Quel est donc ce pretium? $\mathrm{Vu}$ le rapport qui existe entre les paragraphes 1,2 et 1,3 , il doit s'agir du pretium partis dimidiae ad fiscum pertinentis, ce qui pose un nouveau problème: que représente cette pars dimidia ad fiscum pertinens? S'agit-il de la moitié du minerai extrait ou de la moitié du puits?

\section{1 — «Pars dimidia ad fiscum pertinens». «Pars occupatoris». «Puteus universus».}

On peut d'abord considérer que cette moitié est celle du minerai extrait. Ainsi fait $\mathrm{Cuq}\left({ }^{171}\right)$, selon qui le fisc a droit à la moitié du minerai sorti du puits mais qui, ne désirant pas que ce dernier lui soit livré en nature, en réclame le prix, selon un tarif que nous ne connaissons pas.

Or, en fondant du minerai avant que le fisc ait pu connaître quelle en était la quantité totale et par conséquent avant d'avoir payé à ce dernier le prix de cette moitié qui lui revient, l'occupant a voulu frauder. S'il est convaincu de ce fait, on Je punit en confisquant sa part de minerai et tout le puits est vendu par le procurateur. On ne manquera pas de s'étonner de l'énorme disproportion qui existe entre l'ampleur de cette dernière sanction et Je caractère relativement véniel de l'infraction commise: au paragraphe 9, la dissimulation de minerai, qui en fait a pour but la fusion illicite de ce dernier, sera punie d'une forte amende (1 000 sesterces), mais qui n'a rien de commun avec la sanction précédente, alors que, si l'on interprète comme Cuq la dimidia pars ad fiscum pertinens comme étant une part de mineiai, il s'agirait de deux cas très voisins.

(in) CUQ, CR AT, 1907, p. 96; Règlement, p. 110; Le développement de Vindustrie minière, p. 296-299; VENDEUVRE, p. 23 et 118. MAGUEIJO, p. 140 adopte la position de CuQ.

Conimbriga, 22 (1983), 5-193 
Aussi convient-il d'examiner s'il n'est pas plus satisfaisant de voir dans la pars dimidia ad fiscum pertinens la moitié du puits luimême. Si cette pars dimidia ad fiscum pertinens est une moitié de puits $\left({ }^{172}\right)$, il s'ensuit que l'autre moitié est la pars occupatoris, le tout formant le puteus universus. Ce sont là sans doute des notions juridiques, mais à quoi correspondent-elles? Nous avons effleuré plus haut cette question dans notre commentaire de Vip. /, 9. Le puteus, avons-nous dit, ne pouvait exister que, d'une paît, grâce au sol dans lequel il était creusé et qui, comme le sous-sol qu'il recouvre et qui n'en est juridiquement pas distinct, appartient à l'Etat représenté par le fisc, d'autre part grâce au travail et aux investissements de 1'occupator. Ces derniers servent de fondement à la pars occupatoris, qui représente en quelque sorte les droits propres à 1'occupator et se matérialisera par le puits creusé $A$ équipé. Quant à la pars dimidia ad fiscum pertinens, elle traduit le droit de propriété qui est celui du fisc sur le sol et le sous-sol dans lequel est creusé le puits. Ce puits, que l'on peut considérer comme un instrument d'exploitation du minerai, appartient donc en commun aux deux parties.

Du moins tant qu'il n'a pas atteint la minéralisation. A ce moment là, tout change: si Гoccupator veut exploiter le minerai et en tirer profit en le fondant, il doit auparavant payer au fisc le prix de l'autre moitié. Le fisc lui transfère alors la proprietas

(172) Mispoulet, Tables cTAljustrel, p. 357; Régime des mines, p. 115. Kübler, p. 421; D'Ors, Epigrafía, p. 110; D. Flach, Bergwerksordnung, p. 415-417. Dans notre commentaire de Vip. $/, 9$, il nous avait paru possible de faire la distinction entre le puteus (qu'il s'agît du puits réel ou de l'idée de puits) et la concession qui se définissait comme putsus locusque putei. Dans Vip. //, cette dernière expression n'apparaît jamais. Seul est employé le mot puttus, qui signifie souvent «puits», soit au sens concret, soit au sens juridique du terme (un aspect propre à Vip. II: cf., tout de suite après, ce que nous disons du puteus uniuersus, de la pars occupatoris, etc.), mais aussi parfois «concession», comme dans Vip. I, 18, qui parle de putei determinati et de fines putei. Peut-être cette ambivalence se justifie-t-elle dans la mesure où, de même que le puits ne peut exister sans le terrain dans lequel il est creusé (cf. supra, p. 103), de même, du point de vue minier, la concession ne devient réalité que lorsqu'un puits $y$ est creusé, avec ses galeries, ses chantiers, etc. 
de sa moitié de puits. Sans doute n'en est-il pas vraiment le dominus, puisque dans plusieurs circonstances ( Vip. II, 2 à 5) il peut en être privé. 11 dispose donc d'un droit précaire. Il n'en a pas moins le pouvoir et, dans certaines conditions que vous verrons plus loin, le droit d'exploiter le minerai, que la loi romaine - ne l'oublions pas - considère comme un fruit de la terre, au même titre que les fruits ou les moissons $\left({ }^{173}\right)$. C'est donc une forme de possessio qui est ainsi définie: l'exploitant a l'usufruit d'un sol et d'un sous-sol dont le caractère principal est la richesse en minerai. Cet usufruit pourra s'exercer par l'intermédiaire de l'instrument dont Voccupator est propriétaire, le puits.

Reste à savoir maintenant en quoi consiste le pretium partis dimidiae ad fiscum pertinentis.

\section{2 - La «liberalitas» d'Hadrien et le «pretium partis ad fiscum pertinentis»}

Nous avons vu, dans la première partie de cette étude, qu'outre le cuivre, les gisements d'Aljustrel renferment des métaux précieux, en particulier l'argent. Or Vip. II, 2 concerne les putei argentarii et plus précisément leur prix. Par le biais de cette disposition, nous tenterons de comprendre à quoi correspond le pretium partis dimidiae ad fiscum pertinentis.

D'après la règle exposée dans Vip. II, 1, le seul qui puisse acquérir la deuxième part de puits, celle du fisc, c'est Voccupator. Ici il en va différemment: aura en effet cette deuxième part, celui qui primus pretium puteo fecerit et sestercia quattuor milia nummum fisco intulerit. L'expression pretium puteo facere fait problème: certains, tels Cuq et Magueijo, ont voulu lui attribuer le sens le «procurer de la valeur au puits», «mettre le puits en valeur» (174) ; mais cette interprétation ne nous paraît pas devoir être retenue. Sans doute trouve-t-on l'expression pretium facere dans une pièce

(173) Dig., 24, 3, Sol. Mat., 13 et 14; Paul, Dig., 50, 16, De V.S., 11. Sur la précarité du droit de propriété sur le puits, voir RostovtzeFf, Kolonat, p. 358-359.

${ }^{\left({ }^{174}\right)}$ CuQ, Le développement de vindustrie minière, p. 299-300; MagueIJo, p. 143-145 et 158. Voir aussi YENDEUVRE, p. 118: «donner de la valeur à un terrain aurifère en creusant un puits.» 
de Martial (175), où les corrections de la main même de l'auteur «font le prix» d'un volume de ses Epigrammes. Mais, vu la nature de notre texte, le sens que prend cette même formule dans un autre poème de Martial où il est question du prix offert lors d'une vente aux enchères $\left({ }^{176}\right)$, nous paraît mieux convenir. C'est d'aillens l'interprétation qu'avaient déjà retenue Cagnat et Mispoulet et qu'a récemment adoptée $\mathrm{D}$. Flach $\left({ }^{177}\right)$. La forme selon laquelle se faisait cette offre a été l'objet d'un autre débat: pour les uns $\left({ }^{178}\right)$ c'est au cours d'une vente aux enchères, mais dans ce cas l'adjectif primus ne se comprend pas car, dans une auctio, le plus offrant, qui emporte la vente, est celui qui a parlé le dernier, et non le premier $\left({ }^{179}\right)$.

Si l'on écarte la vente aux enchères, il faut admettre que le prix était fixé à l'avance $\left({ }^{18}\right)$. Le premier qui offrait de le payer et qui versait au fisc 4000 sesterces devenait propriétaire de la pars quae ad fiscum pertinebit. Il y a donc deux actes distincts dans cette procédure: le premier est un acte juridique et administratif (dépôt de l'offre), le second concrétise le premier : il s'agit du versement de 4000 sesterces. On est ainsi conduit à admettre que le prix réclamé pour les putei argentarii était un prix fixe. On a contesté ce point de vue en faisant valoir d'une part que ce prix était bien bas, d'autre part qu'un tel système ne tenait pas compte de la disparité qui devait inévitablement régner entre les puits en ce qui concerne la richesse et la production. On a donc pensé que les 4000 sesterces ne constituaient que le premier versement à valoir sur le prix total dont le paiement devait être

(175) MARTIAL, 7, 17, 8.

(176) MARTIAL, $1,85,7$.

(1771 Cagnat, Règlement, p. 329; Mispoulet, Lex metalli, p. 22 et 29 (avec des passages du Digeste à l'appui) et Tables d'Aljustrel, p. 359; D. FLACH, Bergwerksordnung, p. 418.

(178) KüBLER, p. 422; ROSTOVTZEFF, Kolonat, p. 358. VOELKEL, p. 199.

${ }^{179}$ ) On a aussi imaginé (VOELKEL, p. 199) que le plus offrant était inscrit le premier sur une liste et que c'est par référence à cette liste que le mot primus apparaissait dans Vip. II, 3. Mais c'est là une pure hypothèse car il n'y a, dans l'ensemble de Vip. $I I$, aucune mention d'une telle liste.

$\left.\mathbf{( 1 8 \circ}^{\circ}\right)$ Mispoulet, Tables d'Aljustrel, p. 361; Schonbauer, Geschichte, p. 69-70; D'Ors, Epigrafía, p. 120; D. FLACH, Bergwerksordnung, p. 424.

Conimbriga, 22 (1983), 5-193 
effectué à diverses échéances (181). Malheureusement, rien dans ce qui reste de la loi ne permet d'envisager une telle hypothèse; il $\mathrm{y}$ a même une disposition qui la rend peu vraisemblable. C'est celle du $\S 5$, selon laquelle la moitié du minerai produit par un puits qui, vendu par le fisc puis abandonné pendant six mois, a été repris par un nouvel occupator — nous verrons plus loin dans quelles conditions - doit être réservée au fisc ex more. Cette dernière précision inteidit de croire que le partage du produit du puits était exceptionnel et limité au cas considéré: c'était bien la règle générale ${ }^{(182)}$. Voilà qui justifie l'hypothèse d'un prix d'achat uniforme, étant bien entendu que la moitié de la production était réservée au fisc.

S'agissant de puits d'argent, la libéralité d'Hadrien modifie donc sur un point (qui primus...) la procédure d'acquisition delà pars dimidia ad fiscum pertinens et le prix d'achat lui-même de cette pars. Mais sur le fond la loi n'apporte pas de changement: le pretium partis dimidiae ad fiscum pertinentis est un prix fixe. Il doit donc en aller de même pour les putei visés par le paragraphe 1, spécialement les putei aerarii.

\section{$3-«$ Colonus» et «occupator»}

Le problème du sens de ces mots se pose dès le premier paragraphe. Comme le montre Schonbauer ( $\left.{ }^{183}\right)$, le qui de qui ita non fecerit et conuictus erit... ( $\S 1,2)$, vu le contenu de la phrase qui suit $(\S 1,3)$, ne peut représenter que le mot colonus. Ainsi, en fondant du minerai avant d'avoir payé le prix, un colon a commis une infraction; la sanction est donc pour lui: pars occupatoris commissa esto, dit la loi. On en conclut que cette pars occupatoris était entre les mains du colon, donc que Yoccupator était un colon.

(151) Mispoulet, Tables d'AljaMrel, p. 359-360; D'Ors, Epigrafia, p. 119.

(182) Mispoulet, Tables d'Aljustrel, p. 368; D. Flàch, Bergwerhsordnung, p. 419. SCHONBAUER en revanche limite cette disposition au cas considéré

( $S C H O N B A U E R$, Geschichte, p. 75); mais il pense que le fisc avait institué une taxe à la production (Ibid., p. 93-94).

(188) Schonbauer, Geschichte, p. 77-78.

Conimbriga, 22 (1983), 5-193 
S'il ne semble pas que les deux termes soient interchangeables et que la loi emploie indifféremment l'un ou l'autre ( $\left.{ }^{184}\right)$, il ne faut pas pour autant croire, comme l'a fait $\mathrm{Cuq}\left({ }^{185}\right)$, que ces deux vocables désignent deux sortes d'exploitants ayant des droits différents: les acheteurs de puits (les coloni) et les «occupants» de puits (les occupator es). La plupart des commentateurs ont en effet rejeté avec raison cette interprétation (186).

Il doit cependant y avoir une différence entre les deux termes, mais laquelle? Les colons dont il est ici question ne sauraient être les habitants d'une colonie. Vipasca est loin d'en être une: ce serait tout au plus une bourgade, un uicus comme l'ont restitué les éditeurs en Vip. L d, 1. Pour D'Ors (187), le colonus serait tout simplement «l'habitant» de Vipasca, mais c'est bien vague et d'autre part ce sens est presque exclusivement attesté chez les poètes (188). Aux yeux de Schonbauer ( $\left.{ }^{189}\right)$, de la même façon que, dans l'agriculture, le colonus est l'homme qui laboure, cultive, donc exploite la terre, de même, s'agissant de mines, le colonus ne peut désigner qu'un homme qui exploite ces dernières, un mineur, un exploitant de mines. L'idée est intéressante, mais on y parvient par une acrobatie sémantique reposant sur la seule présence du verbe «exploiter» dans deux formules qui correspondent en fait à deux réalités différentes, car y a-t-il actions plus dissemblables que labourer la terre et exploiter des mines $\left(1^{\circ}\right)$ ?

En fait, si l'on écarte toutes ces solutions ainsi que le sens de «cultivateur» qui n'a rien à faire ici, il semble ne plus rester que celui de «fermier». Ce sens est bien attesté à Rome à la fin de

(184) Mis poulet, Tables d'Aljustrcl, p. 357 et Régime des mines, p. 115-

117. Voir aussi VENDEUVRE, p. 18-31, 107.

(185) CuQ, CRAI 1907, p. 97-99 et Règlement, p. 104-128.

$\left({ }^{18 \mathrm{e}} \mathrm{j}\right.$ Pour les arguments invoqués, voir par exemple Mispoulet, Régime des mines, p. 115-116; Schonbauer, Geschichte, p. 76-79; D. Flach, Rergwerksordnung, p. 417.

(187) D'ORs, Epigrafia, p. 114-117.

(188) TLL, col. 1705.

(189) Schonbauer, Geschichte, p. 77-78.

${ }^{(19 \circ)}$ Voir aussi le point de vue de D. FLACH, Rergwerksordnung, p. 441, pour qui on ne saurait se contenter de la ressemblance formelle entre les deux expressions: agros colere et puteos colere. 
la République et sous le Haut-Empire, mais il est le plus souvent appliqué à la ferme de domaines agricoles (191). Sans doute est-il dérivé de la notion première de «culture» (colere) : le fermier eA celui qui cultive réellement un fonds de terre à la place de celui qui en est le propriétaire. Mais, une fois le mot colonus bien ancré dans l'usage au sens de «fermier», n'a-t-on pas pu l'utiliser aussi à propos d'une activité autre que l'agriculture?

En fait, sous l'Empire romain, le mot colonus a une résonance tout à fait spéciale et fait inévitablement penser aux coloni d'Afrique. Ces coloni-lk sont de petites gens qui, en pratiquant la culture, mettent en valeur les grands domaines impériaux africains, dans des conditions juiidiques qui sont non pas semblables mais comparables à celles des coloni de Vipasca $\left({ }^{192}\right)$. C'est là un caractère qui apparaîtra avec plus de netteté au fur et à mesure que nous avancerons dans l'étude de ce texte, mais dès maintenant, après les travaux de Rostovtzeff, Burian et D. Flach( $\left.{ }^{193}\right)$, ce fait doit être considéré comme acquis. Cela va d'ailleurs nous permettre de mieux cerner la notion d'occupator pai rapport à celle de colonus.

A Vipasca les coloni devaient donc constituer un groupe juridico-social se définissant par une activité minière et métallurgique en vue de la mise en valeur, dans des conditions définies par la loi, des mines impériales de l'endroit.

(191) SE E K, Colonatus, col. 486-487.

${ }^{\left({ }^{192}\right)}$ Sur le colonat dans les grands domaines d'Afrique, voir, outre M. Ros Tovtzeff, Studien zur Geschichte des romischen Kolonates, Leipzig-Berlin, 1910, les études de J. KolEndo, Le colonat en Afrique sous le Haut-Empire, Paris, 1976 et de D. FLACH, Die Pachtbedigungen der Kolonen und die Verwaltung der kaiserlichen Güter in Nordafrika, dans Aufotieg und Niedergang der romischen Welt, 2, 10, 2 , Berlin-New-York (à paraître).

(193) Rostovtzeff，Kolonat, p. 353 sq. ; Burian，Leges, p. 542-543; D. FLACH, Bergwetksordnung, p. 440-446. Ce dernier auteur a plus particulièrement affiné le parallélisme entre les coloni d'Afrique et ceux de Vipasca: sur plusieurs points il $y$ a des rapprochements évidents; en particulier les uns et les autres sont des colon, partiaires (pour Vipasca, voir Vip. II, 5). C'était déjà aussi l'avis de Mispoulet, Tables d'Aljustrel, p. 369. Cependant, quand RostovtzefF, (Kolonat, p. 354-359) fait de Vip. II l'exact parallèle de la lex Hadriana de rudibus agris et considère que le règlement minier ne concerne que les puits abandonnés, il me paraît singulièrement limiter la portée de ce dernier. 
Supposons donc qu'un de ces colons veuille prendre à ferme une concession minière. L'une des procédures pour y parvenir est 1'occupatio. Il y aura recours, dans les formes définies par Vip. /, 9: libre choix de la concession, déclaration auprès du procurateur, paiement du pittaciarium. Du coup il deviendra occupator, ce qui, nous l'avons vu, lui donnera droit à la jouissance du terrain concédé, mais une jouissance d'abord limitée puisqu'au cours d'une première phase, il ne pourra qu'entreprendre le fonçage d'un puits. Nous allons voir dans un instant que la jouissance sera totale lorsqu'une foL atteinte la minéralisation et payée la pars dimidia ad fiscum pertinens, interviendra la phase d'exploitation et de production de minerai, grâce à l'instrument de travail dont il aura acquis la propriété, le puits.

Bref, être colon à Vipasca, c'est jouir d'un statut juridique permanent, celui des entrepreneurs qui, dans des conditions diverses, mettent en valeur les mines impériales. Est occupator le colon qui exploite une concession selon la procédure de 1'occupatio. Autrement dit tous les occupatores sont des coloni, mais l'inverse n'est pas obligatoirement vrai.

\section{4 - Le texte de Vip. //, 1}

Nous pouvons maintenant revenir au texte même du paragraphe 1. Dans la piemière phase tronquée, nous ne compléterons pas [...] Aug. en [proc(uratori)] Aug(usti) comme on l'a lait parfois (194): cette formule désignerait en effet un procurateur équestre, soit un procurateur des mines d'un niveau élévé (piovince ou région) dont la fonction n'est pas autrement attestée dans la Péninsule, soit le procurateur financier de la province, mais on ne voit pas pourquoi c'est à lui, et non aux services financiers locaux comme dans le reste de la loi, qu'il faudrait verser comptant, selon la restitution proposée - [...pretium proc(uratori)] Aug(usti) praesens numerato -, le prix de la moitié du puits appartenant au fisc. Nous considérerions plutôt qu'il pourrait s'agir d'une formule

(194) SCHONBAUER, Gesohichte, p. 103-104; D'ORs, Epigrafía, p. 113-114.

Pour D. FLACH, Bergworksordnung, p. 405, la restitution "procurators est incertaine. 
comparable à celle que l'on trouve au paragraphe 2, secundum liberalitatem sacratissimi Imp(eratoris) Hadriani Aug(usti) (19b), mais d'un contenu différent, car le paragraphe 2 renfermant une exception au régime général due à la liber alitas d'Hadrien, le paragraphe 1 devait se référer à la procédure habituelle de $Y$ occupatio que devait définir pour l'achat des puits de mines de Vipasca une lex impériale antéiieure, qu'il n'y a pas de raison de ne pas attribuer à Hadrien (196). En rassemblant les éléments nous permettant de comprendre le sens de ce premier paragraphe nous avons déjà entrevu les grandes lignes de cette procédure habituelle. Nous examinerons cette dernière plus en détail ci-dessous. Pour l'instant, contentons-nous de dire que, compte tenu des indications données dans les phrases 2 et 3 du paragraphe 1 , le contenu de la phrase tronquée devait être proche de ceci: «...avant de fondre du minerai, le colon qui occupe un puits (= une concession) devra, selon la Loi de l'Empereur Hadrien Auguste, payer comptant le prix de la moitié du puits qui appartient au fisc». Dans le cas contraire, et si par conséquent il est convaincu d'avoir contrevenu aux dispositions précédentes en fondant du minerai avant d'avoir payé le prix de la moitié revenant au fisc, il sera déchu de ses droits d'occupator. Il perdra la pais ocupatoris, autrement dit le puits qu'il a creusé et équipé sera saisi. Et c'est le puits tout entier, le puteus uniuersus, tel que nous l'avons entendu plus haut, que le procurateur mettra en vente, sans doute aux enchères $\left({ }^{197}\right)$, avec l'espoir d'en faire un bon prix en raison de la plus-value prise par la concession grâce aux travaux effectués per le colon occupator et en raison de l'assurance que le puits n'est pas stérile puisque $Y$ occupator a fondu du minerai qui en avait été extrait.

On voit donc que l'infraction au règlement concernant l'acquisition des puits de mines était lourdement punie. Aux yeux de la loi en effet, celui qui la commettait cherchait à frauder le fisc d'une somme importante. En extrayant furtivement du minerai

(195) En ce sens, Bruns, Fontes, p. 293. Voir aussi Rostovtzeff, Kolonat, p. 357,

${ }^{(196)}$ Contra, D'ORS, Epigrafia, p. 113.

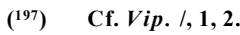

Conimbriga, 22 (1983), 5-193 
et en le fondant clandestinement, le colon pouvait faire croire que le puits qu'il avait creusé n'avait pas atteint de zone minéralisée et, à l'extrême, qu'il était stérile: ce faisant, il ne payait pas le prix de la pars dimidia ad fiscum pertinens et surtout traitait entièrement à son bénéfice le minerai qu'il en extrayait, contrairement à l'usage qui voulait que la moitié du minerai fût réservée au fisc.: ...ita ut cum uenae ex eo proferentur ex more dimidia fisco salua sit (Vip. Il, 5).

La fraude mentionnée dans ce paragraphe devait être fréquente et, malgré les contrôles, difficile à établir. D'où le recours à la délation - qui probauerit ante colonum uenam coxisse quam pretium numerasse... et, réciproquement, qui conuictus erit prius coxisse oenam quam pretium... soluisse — et l'importance de la prime accordée au délateur: le quart de la somme atteinte par la vente du puits.

Nous rappelons brièvement ci-dessous les dispositions générales qui, d'après Vip. IL i, paraissent avoir réglé l'exploitation des concessions minières par occupatio. Par cette procédure qui est déclenchée à l'initiative du colon et qui donne lieu au paiement $\mathrm{du}$ pittaciarium $\left.{ }^{198}\right)$, ledit colon prend possession d'une concession. Ce droit de possession est d'abord limité au creusement du puits d'accès à la minéralisation, ce qui constitue la première phase des travaux et donne droit à la moitié du puits (pars occupatoris). Une fois le minerai atteint, le colon-occupator doit payer comptant (praesens) le prix de la moitié du puits qui appartient au fisc. Dès lors il a l'usufruit de la concession, c'est-à-dire qu'il peut en tirer profit en extrayant du minerai et en le fondant, dans des limites qui ne sont pas clairement énoncées dans la partie $\mathrm{du}$ texte qui nous est conservée mais que le paragraphe 5 nous permettra de définir. Si les clauses énumérées par Vip. $/ /, 1$ ne sont pas respectées, la pars occupatoris (autrement dit le puits en tant qu'objet matériel) sera saisi, et le puits tout entier (puteus uniuersus) vendu aux enchères par le fisc, dans les conditions que nous avons exposées ci-dessus.

${ }^{(198)}$ Cf. Vip. I, 9. 


\section{5 — Les puits argentifères (Vip. II, 2)}

S'agissant des putei argentarii, la liberalitas d'Hadrien semble avoir eu deux effets:

a) les dispositions du régime général (Vip. II, 1) paraissaient favoriser 1' occupator ; ce dernier en effet, s'il observe les prescriptions légales, sait que, si la concession se révèle rentable et s'il décide d'acheter la pars dimidia ad fiscum pertinens, il ne risque pas d'être devancé par un tiers $\left({ }^{199}\right)$. En revanche, désormais pour les putei argentarii le premier colon venu peut acheter cette part: il lui suffit d'en faire l'offre au fisc et de lui verser les 4000 sesterces exigés pour la part du fisc.

b) le prix à payer pour acquérir la proprietas de cette part a été sans aucun doute baissé. Mais reste-t-il supérieur à celui qui est exigé pour la part des autres puits - et en particulier des putei aerarii - qui appartient au fisc, comme le pense D. Flach $\left({ }^{200}\right)$ ?

Nous nous demandons si le contraire n'est pas plus vrai. D'une façon générale on a considéré que les putei argentarii devaient être plus prisés que les putei aerarii (201). Sans doute la valeur de l'argent était-elle plus élevée que celle du cuivre, mais à Vipasca, les puits argentifères ne peulrent avoir été plus recherchés que les puits cuprifères que s'ils étaient plus rémunérateurs que ces derniers. En avons-nous bien la preuve?

Le fait qu'Hadrien n'a pas cherché à favoriser l'exploitation du cuivre montre que cette dernière n'est pas en difficulté. L'acquisition des putei aerarii continue à suivre une procédure normale: tout se passe comme si leur rentabilité était assurée, comme si les échecs étaient rares sinon inexistants; il est donc inutile de «promouvoir» l'exploitation du cuivre par des mesures

(199) Mispoulet, Tables d'Aljustrel, p. 358; D. FlaCh, Bergwerksordnung, p. 415. Ces deux auteurs divergent en ce que le premier pense qu'en tout état de cause $\boldsymbol{Y}$ occupator était tenu d'acheter la part du fisc alors que, pour le second, cette obligation n'existait pas: cela paraît évident dans le cas où la concession est stérile.

$\left(2^{\circ \circ}\right)$ D FLACH, Bergwerksordnung, p. 415.

(201) Outre D. Flach cité à la note précédente, voir Mispoulet, Tables d'Aljustrel, p. 360-361 et D'ORS, Epigrafía, p. 119. Contra, VendeUVRe, p. 124. 
exceptionnelles; il faut au contraire rassuier les colons et effectivement la procédure normale est rassurante, qui leur garantit la propriété totale du puits dans la mesure où ils auront souscrit aux exigences de la loi. La certitude du profit permet donc de maintenir le prix des puits de cuivre à un niveau élevé.

En revanche pour les puits d'argent, il en va autrement. $\mathrm{Si}$ leur prix est en baisse, si on les soustrait à la procédure normale - nul besoin d'être déjà occupator, donc d'avoir payé la taxe du pittaciarium ( Vip. /, 9), pour pouvoir acheter la part du fisc -, c'est qu'ils attirent peu les colons. On en déduira deux conséquences. La première est que le gisement est pauvre en minerais argentifères ou du moins que leur distribution est capricieuse et qu'ils sont difficiles à découvrir. De fait des indices semblent montrer qu'à Vipasca les minerais de cuivre étaient plus communs que les minerais d'argent: d'une part on pouvait trouver des oxydes sinon sur toute la hauteur, de moins sur une bonne partie du chapeau de fer, et de plus en plus à mesure qu'on s'y enfonçait jusqu'à rencontrer la riche couche de sulfures à la base de ce dernier; en revanche les minerais d'argent ne constituaient qu'une mince strate $\left({ }^{202}\right)$ entre la zone d'oxydation et la zone de cémentation (sulfures de cuivre secondaires); d'autre part, si l'on tient compte des analyses de scories, celles qui semblent provenir de la métallurgie de l'argent sont rares $\left({ }^{203}\right)$. A Vipasca donc les minerais d'argent ne devaient pas abonder. Dès lors on conçoit que pour en favoriser la recherche l'Empereur ait assoupli les conditions d'exploitation. La deuxième conséquence c'est que l'Etat avait besoin de ce métal, sans doute pour des raisons d'ordre financier et monétaire. En effet la mesure qui à Vipasca est due à la libcralitas d'Hadrien ne se justifie que si elle entre dans une politique globale de pioduction de l'argent: vu la ressemblance entre ce gisement et celui de Riotinto, qui, lui, semble avoir

(202) Bien qu'à Aljustrel les recherches sur la présence de ces minerais n'aient pas été aussi poussées qu'à Riotinto où le gisement est comparable (cf. Salkield, Ancient slags, p. 85-98), il semble bien qu'ils aient été plus rares dans la mine portugaise.

(203) Voir supra, p. 29, note 59. Mais dans ce domaine aussi les recherches ont été peu développées, s'agissant d'Aljustrel. 
produit beaucoup d'argent, l'administration romaine a sans doute tenté de voir si véritablement le metallum Vipascense n'était pas lui aussi capable de produire de l'argent.

Mais comment, en pratique, tout cela devait-il se passer? $\mathrm{Si}$, comme le prévoit la loi, un colon devenait propriétaire de la part $\mathrm{du}$ puits qui appartient au fisc, allait-il entrer en conflit avec $Y$ occupator? former avec lui une sociedad involuntaria $\left.Y^{2,0 *}\right)^{\prime}$ ? Le contraindre à lui vendre sa pars occupatoris ? Sans doute rien de tout cela, car si, entre autres conséquences pratiques, la mesure d'Hadrien a pour effet de supprimer, pour la recherche de l'argent, la taxe du pittaciarium, c'est qu'aucun colon de Yipasca ne se portait occupator d'un puteus locusque putei en vue d'exploiter ce métal.

Deux points restent à préciser: on a contesté que le fisc transmette à l'acheteur la proprietas de sa part de puits (205). L'interprétation que nous avons donnée plus haut du puteus considéré comme instrument implique fort bien que le colon puisse en devenir seul propriétaire; en revanche rien dans le texte ne permet de supposer que le fisc aliène la propriété d'une partie du domaine impérial, d'un locus putei; bien au contraire, et c'est ce qui lui permet de prétendre à la moitié du minerai extrait. Quant au colon propriétaire d'un puits, il pouvait être déchu de son droit de propriété dans les conditions qui sont précisées dans les paragraphes suivants.

Enfin la formule qui... pretium puteo fecerit paraît à première vue ne pas s'accorder avec le sens général de la fin du paragraphe, selon laquelle aura la propriété de la part du fisc celui qui le premier offrira le prix pour le puits: ou bien l'emploi du seul mot puteus est un raccourci commode poui éviter la répétition d'une formule aussi longue que pars ad fiscum pertinens et il traduit aussi l'impression qu'avaient les coloni de payer en fait le puits entier en versant une somme qui au regard de la loi, ne représentait que le prix d'une moitié ou bien il signifie que le colon a accepté le prix total du puits tel qu'il a été fixé à

(204) D'Ors, Epigrafia, p. 120.

$\left({ }^{2}{ }^{\circ}\right)$ D. F LACH, Bergwerksordnung, p. 415. 
l'avance par le fisc (c'est la formalité administrative) étant bien entendu que, vu l'existence de la pars occupatoris, il n'en paiera que la moitié, correspondant à la dimidia pars ad fiscum pertinens.

Comme l'a fait remarquer Schonbauer (206), ce paragraphe est par son style différent des autres. En particulier les impératifs par lesquels s'expriment ailleurs les dispositions de la loi $\left({ }^{207}\right)$ font place ici à une autre tournure où est employé le verbe debere. Ce changement de style semble indiquer que Vip. //, 2 a été inséré parmi des dispositions antérieures: ainsi la mesure due à la libéralité d'Hadrien serait bien une mesure ponctuelle. La date à laquelle elle a été introduite parmi les autres dispositions est à placer dans le cours du règne d'Hadrien, puisque ce dernier n'est pas appelé diuus.

\section{b) $\S 3$ à 5: $\quad$ Conditions d'exploitation des puits par «occupa-} tio». Caducité des droits

Les paragraphes 3 et 4 concernent deux cas où le colonoccupator est déchu de ses droits sur des puits qui n'en sont encore qu'au stade préparatoire. Gomme on l'a vu (Vip. //, 1), cette étape est celle des travaux qui préludent à l'exploitation même $\mathrm{du}$ minerai. Quelque désireux que soit le fisc de voir un puits «occupé» devenir productif (deuxième étape de 1 exploitation) le plus rapidement possible, il tient compte des nécessités qui pèsent sur l'exploitant. Ainsi (Vip. //, 3), il comprend que celui qui «occupe» plusieurs puits, c'est-à-dire plusieurs concessions, a besoin d'obtenir rapidement des résultats pour couvrir ses frais, et, dans cette intention, concentre ses efforts sur quelques-unes seulement de ces concessions: au moins une sur cinq, dit la loi( $\left.{ }^{208}\right)$.

(206) SCHONBAUER, Geschichte, p. 64.

(207) A l'exception également de la première partie de Vip. II, 9, qui est peut-être aussi une adjonction.

${ }^{(208)}$ Cf. D'ORS, Epigrafía, p. 121; D. FLACH, Bergwerksordnung, p. 426. Contra, CUQ, Le développement de Vindustrie minière, p. 352, qui croit que cinq était le nombre maximum de puits que pouvait foncer un exploitant, ainsi que Schonbauer, Geschichte, p. 72-73, selon qui personne ne pouvait occuper plus de cinq puits. Mispoulet, Tables d'Aljustrel, p. 365, pense que cinq est un nombre minimum. 
Mais dès que ces premiers puits auront été creusés et deviendront productifs, l'exploitant devra attaquer les autres concessions, faute de quoi, il perdra le droit à Yoccupatio qui s'attache à ces dernières et, à son tour, un tiers aura le droit de les occuper. Par ailleurs, pour entreprendre les travaux, des capitaux sont indispensables. La loi accorde ( $(4)$ un délai de vingt-cinq jours pour réunir ces derniers. Ce délai une fois écoulé, si les travaux ne sont pas commencés sur le champ et si par la suite, il y a un arrêt de plus de dix jours consécutifs, ['exploitant perd son droit sur le puits et un tiers peut occuper ce dernier.

Le paragraphe 5 a pour objet le cas d'un puits vendu par le fisc et qui reste six mois de suite inactif, à la suite de quoi l'exploitant est déchu de son droit. Nous ne connaissons, d'après ce qui nous îeste de la loi, qu'une seule circonstance où un puits est vendu par le fisc: c'est (Vip. //, 1) lorsqu'un colon a fondu $\mathrm{du}$ minerai avant d'avoir payé la part du puits appartenant au fisc; la pars occupatoris est saisie et le puteus uniuersus est vendu par le procurateur. On sait ainsi que la concession n'est pas stérile puisque le puits a produit du minerai. Pourquoi dans ces conditions la loi admet-elle qu'il puisse rester inactif pendant six mois consécutifs? Il faut pour le comprendre, situer ce cas dans la réalité de l'exploitation. Au moment où il est vendu par le fisc, le puits est capable de produire, et l'on imagine mal dans ces conditions que son acquéreur le laisse inactif. Bien au contraire, il en extrait du minerai pour son plus grand profit et celui du fisc. Mais vient un jour où la veine s'épuise et où les recherches pour en découvrir une nouvelle s'avèrent vaines. Dès lors l'exploitant peut négliger le puits et le fisc considère que, s'il le laisse inactif pendant six mois consécutifs, c'est qu'il a renoncé désormais à l'exploiter: tel est en tout cas Je délai retenu par la loi pour déchoir le colon de son droit de propriété.

Dans Jes cas envisagés par Vip. II 3 et 4, malgré l'attention apportée à la situation des occupatores, le souci du rendement (209) J'emporte, car il y est question de concessions vierges dont on ignore encore la richesse mais qu'il faut tester au plus tôt, d'où

$\left(2^{\circ 9}\right)$ Point de vue semblable dans D. FLACH, Bergiverksordnung, p. 426. 
la brièveté des interruptions que la loi tolère dans ravancement des travaux. Il n'en va pas de même pour le type de puits dont s'occupe Vip. //, 5; il s'agit là d'un puits exploité depuis longtemps, qui paraît épuisé et que l'on ne peut pas enlever à son propriétaire avant de lui avoir laissé le temps (six mois) de tout tenter pour retrouver la minéralisation.

Dans tous les cas de déchéance examinés par Vip. II, 3 à 5 , le droit d'occupatio est transféré au colon qui prend la succession de l'exploitant. Il semble bien qu'il l'acquière gratuitement, c'est-à-dire, par exemple pour les cas évoqués aux paragraphes 3 et 4, qu'au stade où en sont les travaux - puits encore non commencé $(\$ 3)$ ou en cours de fonçage (§ 4) - le nouvel occupator n'a pas à acquitter le pittaciarium que son prédécesseur a déjà payé. La formulation de Vip. II, 5 incline à le penser: un tiers a le droit d'occuper un puits vendu par le fisc puis inactif pendant six mois dans des conditions telles que, si du minerai en est extrait, la moitié en sera réservée au fisc ex more. La précision est importante non seulement en raison de la présence de cette dernière formule que nous avons déjà commentée plus haut $(210)$ et qui prouve l'existence d'un colonat partiaire à Vipasca, mais aussi parce qu'elle implique que la seule charge qui pèsera sur le nouvel occupant sera la remise au fisc de la moitié du minerai éventuellement $\left({ }^{2} \mathrm{U}\right)$ extrait du vieux puits, à l'exclusion des droits (pittaciarium) et des versements (pretium partis dimidiae ad fiscum

$\left(21^{\circ}\right)$ Voir supra, p. 128 et 133.

(211) Eventuellement, car l'abandon du puits par l'exploitant précédent peut faire craindre qu'il ne soit désormais stérile.

Le problème se pose de savoir pourquoi le fisc se réserve la moitié du produit d'un puits en minerai et non en métal. La deuxième solution paraît en effet plus pratique, mais peut-être la fraude était-elle alors plus facile. En tout cas c'est de minerai qu'il s'agit: dès lors ou bien le fisc dispose d'usines (officinae) pour traite: ${ }^{1}$ le minerai, ou bien, au vu du minerai extrait, entassé près du puits et sans doute classé par catégories, le fisc exige non pas $50 \%$ du minerai en nature mais son équivalent en monnaie. Cette deuxième hypothèse nous paraît la meilleure: il est peu probable en effet que le fisc ait ses propres usines; s'il confie l'exploitation des mines du domaine impérial à des colons, c'est pour ne pas avoir à la gérer. Le même souci devait prévaloir pour la fusion du minerai. 
pertinentis) dont il aurait dû s'acquitter s'il avait occupé le puteus locusque putei dès le début. Mesure équitable sans doute si l'on songe que le pittaciarium a été effectivement payé par le premier occupator et que la vente aux enchères a dû rapporter au fisc bien davantage que le montant de la pars dimidia ad fiscum pertinens. Mais, soucieux d'accroître toujours davantage ses profits, le fisc aurait pu, à chaque fois, exiger le paiement des taxes et les versements réglementaires. S'il ne l'a pas fait, c'est qu'il n'avait pas intérêt à pressurer exagérément les colons. Sans doute les nouveaux exploitants de ces puits bénéficiaient-ils du ius occupandi dans des conditions somme toute favorables, mais ils n'en étaient pas moins soumis aux taxes, redevances et auties versements qu'impliquait, dans ce cadre juridique, la poursuite de Fexploitation à partir du moment où ils prenaient cette dernière. Ainsi le ius occupandi était étendu aux successeurs des premiers occupatores dans des conditions assez libérales; il est donc probable que les acheteurs des putei vendus par le fisc ( Vip. II 1) en jouissaient eux aussi dans des formes comparables, bien que le texte qui nous est parvenu n'en dise rien.

Les trois paragraphes que nous venons d'analyser nous paraissent obéir à quatre impératifs: ${ }^{1}$

1 - ne pas laisser les travaux se ralentir, et, pour cela, tenir les entrepreneurs en haleine; maintenir sinon élever le niveau de la production.

2 - éviter que les puits ne soient accaparés par un petit nombre de colons - ce qui favoriserait le ralentissement de Fictivité — et que ne se forment de trop grandes entreprises qui auraient tendance à occuper de nombreux puits et à les garder en réserve sans les travailler; parallèlement, multiplier Je nombre de coloni-occupator es.

3 - favoriser les intérêts du fisc, en développant l'activité, source de taxes et de profits divers, en multipliant les points d'extraction du minerai, en exerçant un contrôle strict sur les délais accordés aux fermiers, enfin en maintenant ses droits même sur des concessions abandonnées.

4 - ne pas pressurer exagérément les colons: quand les taxes réglementaires ont été déjà payées, les nouveaux occupants n'ont pas à les acquitter une seconde fois. 
peuvent guère être que les partes occupatoris. Mais pourquoi la loi n'éprouve-t-elle pas le besoin de parler de celles-ci et insiste-t-elle au contraire sur les premières? Il s'agit ici de ventes faites par des colons, et ces derniers ne sauraient vendre que ce qui leur appartient: c'est clair, nous l'avons vu, pour la pars occupatoris puisqu'elle représente le travail du colonus-occupator. Il est donc normal - et c'est ce que considère la loi en n'en parlant pas que le colon en dispose à sa guise. Mais la chose pouvait être moins claire pour la pars dimidia ad fiscum pertinens: tel risquait de croire que le fisc conservait sur elle quelque droit, même après qu'il l'avait vendue en bonne et due forme à $Y$ occupator ou, dans le cas de putei argentarii, à celui qui le premier en avait offert le prix et payé au fisc 4000 sesterces ( II, En fait, comme le dit la loi, le colon qui achetait la part du fisc en avait la propriété. Mais le rédacteur de la loi a sans doute pensé que la chose avait besoin d'être précisée: ces partes puteorum, une fois qu'elles avaient été achetées art fisc et que leur prix avait été payé, étaient bien la propriété des colons-acheteurs. Elles pouvaient donc, elles aussi \{quoque), être vendues à condition de rester entre les mains de colons.

Ainsi donc ces derniers pouvaient se vendre l'un à l'autre, selon toute vraisemblance, les partes occupatoris et aussi les partes puteorum achetées au fisc et dûment payées, à savoir les partes dimidiae ad fiscum pertinentes. Du coup ce sont aussi des puits entiers ( puteiuniuersi) qui pouvaient faire l'objet de ces ventes, y compris, sans doute, ceux qui avaient été saisis et vendus par le fisc, puisqu'aussi bien la pars ad fiscum pertinens de ces derniers avait été achetée et payée par le premier occupator (Vip. Il, 1 et 5). Enfin, ainsi mis sur le marché par la procédure de Yoccupatio ou par la vente à l'initiative du fisc, ces puits et ces parts ou, plus précisément, ces moitiés de puits étaient ensuite susceptibles d'être revendus indéfiniment.

Est-ce à diie que désormais ils vont se vendre sous la forme impliquée par la distinction juridique que nous venons de rappeler entre pars occupatoris et pars ad fiscum pertinens? C'est peu probable. Sans doute était-il possible qu'un colon achetât une moitié de puits, mais ce n'est pas parce que les putei se vendaient en deux partes dimidiae représentant la part de Yoccupator et celle 
que la formule ne soit pas très explicite, iJ faut sans doute entendre des dépenses dont les associés peuvent critiquer l'utilité mais qui ont été faites de bonne foi. Dans les deux cas (213) l'auteur des dépenses a droit à être remboursé par ses associés pro sua quisque portione. Si ces derniers refusent, la loi met à sa disposition les moyens de faire respecter son droit. Une procédure en trois étapes est prévue dans le premier cas alors que rien n'est indiqué pour le second. Cette procédure est la suivante: pendant trois jours, à l'endroit le plus fréquenté du forum, affichage d'un état des dépenses: sommation faite par le crieur public auprès de chaque associé récalcitrant; en cas de non-exécution, saisie de la part de puits revenant à ce dernier (puisqu'en fin de compte une part d'associé représente une part de puits) et partage de cette part entre les différents associés participant aux dépenses.

\section{d) §8: Cession des parts de puits}

Comme nous l'avons déjà dit, à Vipasca les colons constituent un groupe juridico-technico-social bien défini. Nous en avons la preuve ici : c'est entre eux que les colons se revendent les parts de puits. Et ils peuvent le faire aussi cher qu'ils voudront : c'est là un domaine dans lequel le fisc n'intervenient pas.

$\mathrm{La}$ formule colonis inter se eas... partes puteorum uendere peut laisser penser que les ventes de parts de puits concernées par Vip. //, 8 étaient des ventes privées, effectuées de gré à gré. Il semble en effet que dans une auctio des gens qui n'étaient pas des colons auraient pu se porter acquéreurs, ce qui ne s'accorderait pas avec les termes mêmes de la loi. Mais pourquoi ne pas admettre que, dans une auctio organisée pour la vente de parts de puits, seuls les colons avaient le droit d'enchérir (214) ? En tout cas, même

(213) Au début du paragraphe 7, et au sens de «et de plus»: de plus les associés doivent participer aux dépenses qui ont été faites de bonne foi.

${ }^{(214)}$ Colonis inter se eas quoque partes puteorum... uendere : on a là un argument supplémentaire pour soutenir, comme nous le faisons, p. 130 et ici même, qu'à Vipasca, et d'une façon générale dans les mines impériales, les colons constituaient une classe technico-socio-économique dont l'existence 
si l'on considère que Vip. //, 8 concerne des ventes de parts de puits de gré à gré, on doit bien remarquer que la loi dit simplement qu'une telle procédure est permise aux colons (215). Elle n'exclut pas expressément Yauctio dont, pour notre part, nous admettrons fort bien la possibilité, à la condition que nous venons d'évoquer.

Considérons maintenant le quoque que nous trouvons dans ce début du paragraphe 8: colonis inter se eas quoque partes puteorum quas a fisco emerint et pretium soluerint uendere quanti quis potuerit liceto. Il semble signifier que des parts de puits autres que celles qui sont ici nommément désignées et dont 1a. loi n'avait pas besoin de parler faisaient naturellement l'objet de ventes entre colons. Quelles pourraient-elles bien être ? Pour répondre à cette question, il faut d'abord identifier celles que la loi mentionne expressément. Or ces dernières semblent bien être les partes dimidiae ad fiscum pertinentes telles que nous avons pu les définir plus haut en commentant les dispositions de Vip. //, 1 et 2, auxquelles font écho les formules ici employées: eas... partes puteorum quas a fisco emerint et pretium soluerint. Dans ce cas les autres parts de puits ne

garantissait au fisc la bonne exploitation du domaine minier. Les putei devaient donc rester entre leurs mains.

Or le problème que nous venons de poser à propos d'une vente de puits ptr auctionem n'aurait pu manquer de surgir dans les auctiones pour la vente des puits mis sur le marché par le fisc (cf. Vip. I, 1 et 2). Que ces ventes aient existé montre qu'en fait ce problème était résolu, sinon c'est la loi elle-même qui aurait rendu possible la «fuite» des puits hors du cercle des colons. De quelle façon? Peut-être, comme nous le suggérons ci-dessus, par une prescription réservant aux colons le droit d'enchérir. A moins que ce ne soit là pour un simple particulier une des façons de devenir colon, car, si par Vip. II nous pouvons assez bien nous représenter ce qu'était la condition des colons de Vipasca, rien ne nous permet de savoir comment on le devenait.

Í215) On conçoit en effet que les colons aient pu, dans certains cas, préférer la vente de gré à gré, non grevée par des taxes, à la vente aux enchères où la marchandise pouvait atteindre un prix plus élevé, mais duquel il fallait soustraire le montant des impôts (celui du centième) et des redevances diverses (par exemple merces de $Y$ argentarius, du praeco, etc.). Par ailleurs, pour favoriser l'activité économique, le fisc aurait bien pu autoriser ce type de vente, bien qu'à la différence de Yauctio, elle ne lui rapportât rien. 
Après Texception que constitue Vip. II, 2, les paragraphes qui suivent renouent avec l'impératif qui est le mode favori de la loi. On remarquera la formule sicut supra scriptum est, qui rappelle celle de Vip. II, 1 et qui, unie au style impératif, permet de rattacher Vip. II, 3 et ceux qui suivent aux dispositions de la loi antérieures à Vip. II, 2.

\section{c) $\S 6$ et 7: Formation et fonctionnement des sociétés destinées à faciliter le financement des opérations minières}

Les travaux qui échoient d'emblée à Yoccupator - creuser le puits et les galeries permettant d'accéder à la minéralisation entraînent des dépenses considérables, que vient gonfler encore le paiement du prix de la moitié du puits appartenant au fisc ( $\S 1$ et 2). S'il ne pouvait faire face seul à de tels frais, la loi lui donnait, entre autres possibilités $(212)$, celle de rassembler les capitaux nécessaires en constituant une société.

La société dont il est question aux paragraphes 6 et 7 est apparemment une société ordinaire, du type unius rei, constituée pour l'exploitation d'un puits. Y?occupator choisira ses associés à sa guise (quos volet). Il y aura apport réciproque des associés (pro ea parte qua quis socius erit), et ceux-ci devront participer aux dépenses à proportion de leur apport.

Dans le cas contraire, la défense des intérêts de chaque associé est assurée par diverses mesures, auxquelles se réfèient Vip. II, 6 et Vip. II, 7. Le problème fondamental est celui des dépenses qui ont été engagées dans un puits exploité par Yoccupator et ses associés. Dans quelle mesure sont-ils tenus de participer aux dépenses faites par l'un d'eux dans ledit puits, et, s'ils refusent, quelles sont les sanctions? Il semble bien qu'ici le législateur distingue d'une part les dépenses que l'on peut justifier en en produisant l'état [ratio impensarum), qui sont par conséquent indiscutables et que les associés ne sauraient refuser de payer, d'autre part celles qui ont été faites de bonne foi (bona fide) : bien

(2i2) D'ORs, Epigrafia, p. 124.

Conimbriga, 22 (1983), 5-193 
du fisc; simplement cet achat correspondait aux possibilités financières du colon. Et c'est là toute la question. On en a la preuve lorsque Vip. II, 6,1 à 3 traite de la formation d'une société pour l'exploitation d'un puits: chacun des associés devient propriétaire d'une part de ce puits (pars putei) au prorata de son apport. Cela implique donc des parts d'importance variable et ce sont celles-là que l'on trouvait sur le marché.

Bref, dans Vip. II,8, le mot pars nous paraît avoir deux sens: dans Vip. II, 8, 1 les partes puteorum sont des parts juridiques, nées de la procédure de 1' occupaproprement les partes dimidiae ad fiscum pertinentes, dont l'achat et le paiement par les coloni-occupatores avant toute exploitation proprement dite (abattage et fusion de minerai) conditionnent une fois pour toutes leur mise sur Je marché et, partant, celle des puits entiers. En revanche quand Vip. II, 8, 2 parle des colons qui vendent leur part ( parlem) ou qui sont susceptibles d'en faire donation, il s'agit de parts réelles, d'importance diverse et dont la grandeur varie simplement en fonction de la somme d'argent investie par eux dans les sociétés constituées pour l'exploitation des concessions; c'est encore le cas de la part de puits que se partagent des associés et qui appartenait à l'un des leurs désormais déchu de ses dioits (Vip. II, 6, 3). Vip. II, 8, 1 établit les conditions auxquelles des puits sont mis sur le marché: il faut que la pars dimidia ad fiscum pertinens ait été achetée et payée au fisc. Après quoi, étant entendu que désormais les prix desdits puits sont libres, Vip. II, 8, 2 fixe les modalités selon lesquelles les ventes de parts de puits appartenant aux colons seront valables: une déclaration devra être faite au fisc par le vendeur et par l'acheteur.

$\mathrm{Y}$ avait-il des puits exclus de la vente? Nous l'ignorons: sans doute pourrait-on penser que les putei adsignati mentionnés par Vip. II, 18 en faisaient partie, car leur appellation même suggère qu'ils étaient attribués gratuitement par le fisc à des colons, qui, de ce fait, n'en étaient pas propriétaires. Mais nous ne savons rien des modalités juridiques qui étaient la conséquence de cette adsignatio, ce qui nous empêche de nous prononcer sur ce point.

Quoi qu'il en soit, dans le cadre des dispositions prévues par Vip. II, 8, 1, le marché des puits est libre. Les colons peuvent se les vendre entre eux aussi cher que possible. Si les prix montent,

Conimbriga, 22 (1983), 5-193

ío 
c'est que l'activité est florissante. La liberté des prix des puits est un signe de bonne santé pour le metallum. Mais tout cela dans les limites définies par le fisc. Ainsi ce dernier garde toujours le contrôle de l'exploitation : les transactions sur les puits sont libres, mais ceux-ci devront auparavant avoir été mis sur le marché par le fisc, donc achetés et payés au fisc dans les formes prévues par la loi. Par ailleurs vendeurs et acheteurs devront faire une déclaration au procurateur, faute de quoi les transactions ne seront pas valables: il importe en effet que le fisc sache à qui réclamer sa part de minerai. Enfin la donation d'une part de puits est illicite, si le donateur est débiteur du fisc.

\section{e) $\$ 9$ et 10: $\quad$ Conditions de traitement du minerai. Dissi- mulation et vol}

Ces deux paragraphes dont la place a pu paraître incongrue à certains (216) n'ont pas de quoi surprendre. Ils concernent la dissimulation et le vol de minerai, deux délits dont le fisc risque toujours d'être une victime, dans la mesure où, comme nous le savons $(217)$, il se réserve la moitié du minerai extrait. Ils sont donc sur le même plan que Vip. //, 1 et $2, k$ ceci près qu'ils concernent la phase de production, et il n'est pas anormal de voir ces problèmes abordés après ceux qui concernent la phase préparatoire des travaux (Vip. //, 1 à 5), les sociétés constituées par les colons pour l'exploitation (Vip. //, 5 et 7) et la cession des parts de puits (Vip. II, 8). Avec le paragraphe suivant en effet commenceront les questions techniques.

Le paragraphe 9 précise que le minerai devra être transporté de la mine aux usines pendant la journée. Le transport de nuit est interdit. Quiconque enfreindra cette prescription sera puni d'une amende de 1000 sesterces. Si la loi interdit de transporter pendant

(216) Schonbauer, Geschichte, p. 92-94.

(217) Cf. Vip. //, 5.

Conimbriga, 22 (1983), 5-193 
la nuit du minerai aux usines, c'est évidemment pour éviter la fraude dont le fisc serait la victime: il tient en effet à ce que le pourcentage auquel il a droit soit calculé sur la quantité totale de minerai. Au paragraphe 10 c'est le vol de minerai qui est visé.

Une amende de 1000 sesterces frappe la personne convaincue de dissimulation; les peines prévues à l'égard des esclaves et des hommes libres coupables de vol sont lourdes: fouet, vente ordonnée par le procurateur, port de chaînes, interdiction de séjour dans toutes les mines et dans tous les districts miniers pour les premiers, confiscation des biens, interdiction de séjour sans doute sur le territoire du metallum Vipascense pour les seconds $\left({ }^{218}\right)$. La rigueur de ces peines est extraordinaire et ne s'explique que parce que l'intérêt du fisc est en jeu.

Par ailleurs une des conclusions à tirer de ces paragraphes, c'est que les usines (officinae) dont il est question ici étaient des usines privées. S'il s'était agi d'installations publiques, le fisc eût pu exercer facilement un contrôle sur leur activité, et en particulier sur les entrées de minerai. Dès lors il eût été inutile de surveiller les transports de minerai $\left({ }^{219}\right)$.

(218) Sans doute Ja formule employée - . . finibus metallorum prohibeto manque-t-elle de précision, mais, d'une part, il faut bien qu'elle se différencie de celle qui concerne les esclaves, qui, eux, sont clairement interdits de séjour dans toutes les mines et tous les territoires miniers (...neue in ullis metallis territorisue metallorum moretur); d'autre part, dans Vip. /, 2, l'expression aussi vague intra fines désigne bien le territoire de la mine de Vipasca. Ces deux raisons nous font donc considérer que les hommes libres sont interdits de séjour seulement sur le territoire de la mine de Vipasca (même interprétation pour Vip. //, 13 et 16). Pour la rigueur des peines on a souligné en particulier la gravité de la confiscation (CuQ, Règlement, p. 131-132).

(219) Nous indiquions plus haut qu'à notre avis le fisc recouvrait sa part de minerai non en nature mais en monnaie. Pour traiter le minerai, il eût fallu qu'il disposât d'usines d'Etat: c'est là une notion qui est contraire à l'esprit dans lequel le fisc organise la mise en valeur du domaine public. La remarque que nous venons de faire sur la natuie des usines mentionnées au paragraphe 9 nous paraît renforcer notre point de vue. 


\section{$2^{\circ}$ Mesures techniques concernant la sécurité dans les mines}

Les paragraphes 11 à 18 se divisent en deux groupes concernant, ${ }^{\wedge}$ premier ( $§ 11$ à 13) l'étayage des puits et des chantiers, ainsi

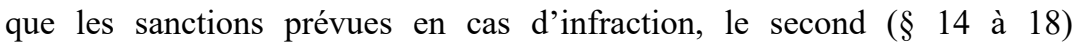
les précautions dont il faut entourer le travers-banc d'exhaure.

\section{a) $\S 11$ à $13-$ Etayage et fortification des puits et chantiers}

A première vue, la formule de Vip. 7/, Ü, colonus cuiusque putei, paraît curieuse. Le mot colonus ne semble pas appeler un complément au génitif de ce genre et de fait il est toujours employé seul dans le reste de la loi. On attendrait plutôt occupator à l'image de Vip. //, 6: Occupatori puteorum, mais, vu la différence que nous avons établie plus haut entre l'occupant et le colon nous ne saurions, à l'imitation de Mispoulet $\left({ }^{22}{ }^{\circ}\right)$, considérer que colonus et occupator sont des mots interchangeables. D'ailleurs, alors que les mesures définies au paragraphe 11 doivent concerner tous les puits, on pourrait se demander pourquoi seuls les putei occupati seraient concernés, alors que Vip. //, 18 nous parle d'une deuxième catégorie, les putei adsignati. Par ailleurs, si plusieurs colons associés (socii) pouvaient participer à la mise en valeur d'un puits, l'un d'eux en était, au regard de la loi, plus proprement responsable: dans le premier cas, c'était $Y$ occupator $_{\eta}$ et les paragraphes antérieurs le montrent clairement, dans le second, ce devait être le bénéficiaire de Yadsignatio. Aussi traduirons-nous colonus cuiusque putei par «le colon de chaque puits» en entendant «le colon responsable de chaque puits».

Il nous semble que chacun des deux premiers paragraphes traite d'un type spécial de fortification. Vip. $/ /, 11$ en effet concerne l'étayage artificiel pour lequel on employait du bois; dans Vip. //, 12, en revanche, les pilae aut fulturae relictae sont les piliers de roche ou de minerai qu'on laisse (relinquere) au milieu des chantiers pour empêcher les éboulements.

(220) Mispoulet, Tables d'Aljustrel, p. 357. 
Si l'on veut que les mineurs ne désertent point la mine, il est essentiel que la sécurité y règne. Il était donc de l'intérêt du fisc que les mines fussent convenablement équipées. Tous les putei et sous ce mot il faut comprendre non seulement les puits d'accès, mais aussi les galeries et les chantiers qui en dépendent doivent être soigneusement étayés et fortifiés (fulti destinatique). Fulcire signifie «étayer» et, du sens premier de destinare, «fixer, assujettir», on tire assez facilement celui de «fortifier» qui, dans l'art des mines, signifie «affermir, assurer par des pièces de bois ou de métal convenablement placées».

Dans le paragraphe 11, il s'agit exclusivement de pièces de bois \{materia) (221). Le colon doit veiller à remplacer ces pièces lorsqu'elles sont pourries par l'humidité qui règne dans la mine: il suffit parfois de cinq ans seulement pour qu'il en soit ainsi. Dans les mines du Sud de la Péninsule Ibérique, le bois communément utilisé était le chêne vert.

Dans le paragraphe 12, il s'agit d'un autre type de fortification: les piliers de roche ou de minerai intentionnellement laissés en place pour supporter le plafond des chantiers et galeries, éviter les éboulements et assurer la libre circulation, d'où les adjectifs appliqués aux pilae fulturaeue, qui doivent être firmae et peruiae. Pila correspond exactement au français actuel «pilier», et, vu le sens général de la phrase, fulturae. qu'il faut évidemment rapprocher de fulcire, ne saurait cependant désigner ici des étais artificiels; il s'agit d'ilots de roche ou de minerai laissés comme piliers de fortification, et la parenté de sens entre les deux mots est indiquée par la conjonction de coordination: pilae aut fulturae. Nous avons traduit ce dernier mot par «supports». Cette technique d'exploitation, qui consiste à abattre le minerai en ne laissant que les piliers nécessaires à la sécurité, était couramment employée dans l'antiquité. En Espagne, lorsque les mines antiques ont été remises en exploitation, au XIX ${ }^{\mathrm{e}}$ siècle, l'abattage des piliers

(221) Voir par exemple les anneaux de boisage polygonaux trouvés dans les mines de Mazarrén (MU 10), les cadres de soutènement de galeries découverts à Riotinto (H 43) et à Herdade do Montinho (POR 11). 
de minerai laissés par les anciens mineurs a souvent constitué la première étape des travaux modernes. On peut penser que, dans l'antiquité également, l'attrait que ces piliers pouvant contenir de riches minerais devaient exercer sur les mineurs risquait de pousser ces derniers sinon à les abattre, du moins à les rogner ou à en ôter les amas de minerai pur, ce qu'indiquent les infinitifs attingere aut uiolare doloue malo quid facere. Il va de soi que tout cela était strictement interdit, car c'était diminuer la force des piliers et multiplier d'autant les risques d'effondrement.

Dans Vip. Il, 13 sont indiquées les peines encourues par ceux qui auront porté atteinte à l'équipement d'un puits et qui auront nui aux conditions de sécurité qui doivent y régner. Le sens des infinitifs uitiare, labefactare ne pose pas de problème. Il en va différemment de celui qui suit. Decapitare signifie en effet proprement «enlever la tête» et, s'agissant d'un être humain, il est employé au sens propre dans la basse latinité. Mais le voici attesté au II ${ }^{\mathrm{e}}$ siècle avec une signification différente. Que veut dire decapitare, lorsqu'il est employé à propos d'un puits de mine? «Oter la tête du puits», sans doute, mais encore. Il ne saurait s'agir d'un échafaudage comparable aux chevalements qui surmontent les puits de mines modernes et qui sont destinés à supporter les système? de descente et de remontée de la cage; des constructions de cette importance paraissent avoir été inconnues dans l'antiquité. Cependant, si la tête (caput) du puits désigne sa partie la plus haute, il doit bien s'agir des «installations superficielles» qui entouraient sa bouche: par exemple, les treuils et équipements divers (amarres pour les câbles) destinés, les uns à remonter le minerai, les autres à faciliter la montée et la descente du personnel; peut-être en outre ces appareils rudimentaires, comme la bouche du puits elle-même, étaient-ils couverts d'un toit. Decapitare signifierait par conséquent «détruire les installations de surface» du puits.

Ici aussi, comme en Vip. Il, 10, les peines sont différentes selon que le coupable sera un homme libre ou un esclave. Pour le premier, on retrouve les mêmes peines qu'au paragraphe 10: confiscation des biens et interdiction perpétuelle de séjour dans le territoire des mines, entendons probablement celles de Vipasca. 
L'esclave sera fouetté à la convenance du procurateur et vendu par son maître; il ne pourra plus être employé dans les mines. Dans les deux cas, les peines sont extrêmement sévères (222).

b) $\S 14$ à 18 - Précautions à observer à Végard du traversbanc d'exhaure

Nous examinerons plus loin les rapports de ip. II avec Vip. Mais il convient déjà de préciser certains points de ce débat. Certains en effet ont pensé que Vip. II était la metallis dicta mentionnée par Vip. I, 9(223) ou du moins une loi analogue, plus récente $\left({ }^{224}\right)$ - en tout cas une loi à caractère général valable pour tout l'Empire ou pour la Péninsule, ou encore pour une de ses provinces $\left({ }^{22 \mathrm{~S}}\right) . \quad 11$ semble au contraire que certains paragraphes s'appliquent étroitement à la mine de $\mathrm{V}$ ipasca et ne peuvent concerner qu'elle. Parmi ceux-ci, nous comptons spécialement les paragraphes 14 à 18 , dont le commentaire, de Cuq à

D. Flach en passant par Mispoulet, Schonbauer, d'Ors et les autres, est toujours extrêmement maigre, à la différence de ce qui se passe pour les dispositions purement juridiques et même pour les paragraphes techniques comme Vip. II, 11 à 13 .

C'est que, même pour ces derniers, les auteurs cités ont pu s'appuyer souvent sur des règlements comparables qui avaient existé en Grèce ou dans le monde hellénistique. En revanche les points de comparaison manquent pour Vip. II, 14 à 18 . C'est peut-être, comme nous allons le voir, parce qu'il s'agit de règles qui d'une part concernent spécialement la mine de Vipasca, d'autre part ne peuvent se comprendre sans référence à la nature particulière des gîtes pyriteux du Sud-ouest dont fait partie Vipasca -Aljustrel.

(222) On remarquera cependant qu'au Laurion, quiconque endommageait les piliers de soutènement était puni de mort (cf. ARdalluon, p. 193; Mispoulet, Tables d'Aljustrel, p. 384).

(223) Mispoulet, Tables d'Aljustrel, p. 353.

(224) ThielmanN, p. 63.

(225) D'OrS, Epigrafía, p. 75 et 111. Cf. aussi vendeuvre p. 7-13. 


\title{
1. Vip. //, 14-18 et la mine d'Aljustrel
}

\author{
a - le «nouum metallum» de Vip. II, 15 et la structure $d u$ \\ gisement des Algares à Aljustrel
}

Nous considérons d'abord le début de la phrase $2 \mathrm{du}$ paragraphe 15: explorandi noui metalli causa. La traduction que l'on donne généralement de cette expression nous semble vague ou inexacte. Il paraît cependant possible de la serrer de plus près. Ainsi on a couramment donné à explorare le sens de "chercher», «chercher en vue de découvrir»: «pour rechercher un nouveau filon» (226), "pratiquer des sondages de recherche» $\left({ }^{227}\right)$, «for the sake of seeking a new mine» $\left({ }^{228}\right)$, «zu Suchzwecken» $\left({ }^{229}\right)$, «for the purpose of discovering new deposits» $\left({ }^{23}\right)$, «for the purpose of discovering new mines» $\left({ }^{231}\right)$, «for the purpose of discovering a new vein» $\left({ }^{232}\right)$. On a donc donné à explorare le sens de exquirere ou perquirere. Il nous semble cependant qu'explorare a un sens plus précis, ou $\mathrm{du}$ moins que le domaine dans lequel s'exerce l'action exprimée est plus limité. $\mathrm{Au}$ sens militaire qui, comme le montre l'article du T. L. L., semble être le premier du mot, ce verbe signifie «faire une reconnaissance», "s'assurer de l'état dans lequel se trouve une zone donnée». Le dérivé français «explorer» nous paraît traduire fidèlement cette notion. Lorsque Stanley, Livingstone ou Brazza explorait l'Afrique Centrale, chacun d'eux connaissait, et pour cause, l'existence de cette région, mais il ignorait ce qu'elle renfermait. Là est la différence entre «chercher» et «explorer»: dans le premier cas, on n'est pas sûr que la chose cherchée existe, dans le second on cherche à connaître une chose dont on est sûr qu'el e existe.

(226) Gagnat, CRAI 1906, p. 331 et Cu Q, Règlement, p. 126.

(227) Mispoulet, Tables TAljustrel, p. 385.

(228) Louis, Mining Laws, p. 40.

(229) Scilonbauer, Geschichte, p. 107.

$\left({ }^{2}{ }^{\circ}\right)$ VAN NOSTRAND, p. 172.

Í231) LEWIS-RHEINHOLD, p. 190.

(232) Cummings-Chalmers-Mattingly, p. 340. 
Traduire... explorandi noui metalli causa... par «zum Zwecke des Erkundens neuer Schachte» (233), par «a fim de explorar nova mina» («explorer une nouvelle mine») (231) ou «zur Erkundung eines neuen Lagers» $\left({ }^{235}\right)$, c'est simplement envisager l'éventuelle découverte d'un nouveau gisement, sans que ce dernier soit une réalité. Pour notre part nous préférons donner à cette expression son sens le plus plein-«pour explorer (ou «reconnaître») le nouveau gîte, - metallum, ayant ici son sens premier de «gîte métallifère» (23e) et nous allons voir que l'étude même du site paraît confirmer notre interprétation.

Quel est donc ce nouveau gîte ? Pour répondre à une telle question, il nous faut rappeler la façon dont se présente à Aljustrel la mine des Algares, celle qui par excellence a été exploitée par les Anciens et près de laquelle ont été trouvées les deux tables de bronze que nous étudions. Nous avons vu en effet ci-dessus (237) que ce gisement a été le coeur de l'exploitation romaine. On y a repéré à l'époque moderne d'importants vestiges de travaux antiques (puits, galeries, chantiers) dans la partie supérieure des masses minéralisées parallèles qui constituent le gisement, le filon $\mathrm{du}$ Mur et, à quelque $150 \mathrm{~m}$ à l'est, le filon du Toit(238). Mais, alors que le filon du Mur est aisément repérable en îaison du puissant chapeau de fer qui le surmonte et forme un «crestón» en saillie de 10 à $15 \mathrm{~m}$ par rapport à la topographie environnante (fig. 2), le filon $\mathrm{du}$ Toit est pratiquement aveugle, du moins si l'on en juge par la partie conservée de son chapeau de fer. $\mathrm{Au}$ sud de la route qui monte aux bureaux de la mine, ce dernier a disparu à la suite de travaux modernes et son emplacement n'est plus marqué que par une longue tranchée aujourd'hui à demicolmatée (fig. 3); au nord en revanche, où il existe encore, une coupe permet d'en distinguer la partie supérieure : large de 5 a $6 \mathrm{~m}$, il est constitué par des oxydes de fer, des brèches et des schistes

(233) Thielmann, p. 275.

(234) MagueiJo, p. 136, 152, 161.

(235) D. FlaCH, Bergwerksordnung, p. 407.

(286) $\mathrm{y}_{0} \mathrm{i}_{\mathrm{r}}$ Domergue, Mines, Première partie. Ailleurs dans le texte, le mot a le sens plus général de «mine».

(237) Voir supra, Livre I, p. 8-30.

(238) pour ces appellations, voir supra, p. 8. 
ferrugineux de couleur brune, mais en surface il ne se signale par aucune saillie (fig. 5): la surface du sol est uniforme, tant sur le chapeau de fer lui-même que sur la roche encaissante et c'est à peine si les schistes verticaux du chapeau de fer sont repérables parmi les débris schisteux brunâtres qui sont épars sur le terrain (fig. 6). $11 \mathrm{y}$ a donc de fortes chances pour que, bien que le chapeau de fer du filon du Toit affleurât au sens géologique du terme, il ait été en fait aussi peu visible autrefois qu'il ne l'est aujourd'hui.

Or, d'une façon générale $\left({ }^{239}\right)$, si dans l'antiquité les filons dont l'affleurement était net ont pour la plupart été repérés et exploités (c'est bien le cas pour le filon du Mur), ceux qui étaient difficilement identifiables ont échappé à l'attention des Anciens et sont restés vierges. Pourtant, à en croire les vieux rapports (240) et à en juger encore aujourd'hui par quelques entonnoirs qui, dans la tranchée moderne à l'emplacement du chapeau de fer du filon $\mathrm{du}$ toit, signalent des puits antiques (fig. 4), ce dernier a bien été exploité par les Anciens, quoique, selon toute vraisemblance, il n'ait pu être découvert à partir de la surface.

\section{b - Le travers-banc (Texhaure et la découverte du mouum metal- lum».}

Comment donc les Anciens ont-ils pu repérer le filon du Toit? Nous croyons tenir la réponse avec le travers-banc d'exhaure (cuniculus qui aquam metallis subducet) mentionné dans les paragraphes 14, 15, 16 et 18 de Vip. IL A Aljustrel, la configuration $\mathrm{du}$ terrain est telle que pour assurer l'évacuation de l'eau des chantiers du filon du Mur - les premiers entrepris et sans doute aussi les seuls existant au début du II ${ }^{\mathrm{e}}$ siècle: nous verrons pourquoi tout à l'heure - il n'y avait qu'une solution: ouvrir un traversbanc dans le vallon de Feitais, à l'est du filon.

${ }^{(239)}$ C'est en tout cas la règle dans la Péninsule Ibérique (voir Domergue, Mines, chap. 2). Première et sixième parties.

$\left({ }^{24}\right)$ RiBeIro et alii, Relatorio, p. 5, signalent que, d'un bout à l'autre $\mathrm{du}$ filon Est ( $=$ filon du Toit)on voyait de nombreux puits antiques, dont certains, dans le secteur sud, étaient encore ouverts et avaient conservé leurs boisages. 
Or plus haut nous croyons ałroir établi (241) que le travers-banc aujourd'hui connu sous le nom de «galeria da Transtagana» (fig. 7) est bien le travers-banc d'exhaure antique. Examinons son tracé tal qu'il est révélé par l'emplacement des puits de jour (fig. 23, 24, 25): il a nécessairement coupé le filon du Toit, et dès lors ce dernier ne peut être que «le nouveau gîte» (nouum metallum) du paragraphe 15 , celui que l'on ne connaissait pas parce qu'il n'était pas visible en surface, celui dont on sait maintenant l'existence mais dont on ignore encore l'extension et qu'il s'agit de reconnaître, d'où l'autorisation exceptionnelle que donne le procurateur pour explorer la mine. Car la loi était impérative: cuniculum ne uiolare liceto. La mesure qui suit est une restriction à cette règle et elle nous paraît traduire une situation nouvelle provoquée par le creusement du travers-banc, à savoir qu'on sait désormais qu'il existe à Vipasca un nouveau gîte mais qu'on en ignore les caractères.

\section{c-Vip. 7/, 75, 2: une adjonction récente}

Nous considérerions donc volontiers que la mesure qui concerne l'exploration du nouum metallum est une adjonction récente au texte tel qu'il nous est parvenu. Car on ne saurait parler éternellement d'un nouum metallum et de son exploration. Si la mesure n'est pas de la même époque que les nouvelles dispositions concernant les puits d'argent dues à Hadrien, elle ne saurait être beaucoup plus ancienne. Il s'ensuivrait que le creusement du travers-banc serait lui aussi un fait assez récent (premier quart du

(24i) Voir supra, p. 154: L'exhaure.

Cuniculo qui aquam metallis subducet: que Ton n'aille pas arguer du temps employé ici, le futur, pour soutenir que le travers-banc d'exhaure avait peut-être été projeté, mais avait pu ne pas être réalisé, donc qu'il n'était pas certain que le travers-banc de la Transtagana fût effectivement antique. Le futur de cette proposition relative est entraîné par les autres futurs de la loi - et en particulier par celui de la proposition dont elle dépend (Qui puteos aerarios aget) - dont la valeur est purement liée au caractère juridique du texte et exprime la portée générale des prescriptions énoncées. Il en va de même pour d'autres relatives telles que, dans vip. 7, 3,1, arbitratu proc. qui metallis praeerit. 
I I e siècle, par exemple) et que l'exploitation du filon du Toit serait postérieure à ce moment.

La façon dont la phrase 2 de Vip. 11, 15 rompt la suite logique des prescriptions introduites par le paragraphe 14 nous semble confirmer notre hypothèse. Supprimons en effet cette phrase. Vip. 11, 14 signale la distance minimum (15 pieds) que les exploitants des puits cuprifères voisins du travers-banc d'exhaure doivent maintenir entre ce dernier et leurs travaux. Comme on vient de nommer le travers-banc, on rappelle brièvement qu'on ne doit pas toucher à ce dernier $(\S 15,1)$. Mais tout de suite après on retrouve les prescriptions concernant les exploitants de puits cuprifères, avec la même distance de 15 pieds à respecter, quand bien même il s'agirait de chercher ou d'abattre du minerai (§ 16). Viennent ensuite les sanctions auxquelles s'exposent ceux qui, malgré cette défense, agiraient contrairement à ces dispositions dans des ternagi (§ 17) qui, vu l'enchaînement des paragraphes, sont des ternagi dépendant des putei aerarii mentionnés plus haut, et n'ont rien à voir avec le ternagus de Vip. $/ /, 15,2$. Si au contraire on remet cette dernière phrase à sa place, on constate qu'elle introduit plusieurs notions et dispositions nouvelles - la question de l'exploration du nouum metallum, la mention d'une galerie de reconnaissance d'un format déterminé, le fait qu'exceptionnellement cette dernière est entreprise à partir du cuniculus qui n'ont aucun lien logique avec celles que renferment les paragraphes 14 et 16-17.

\section{$\mathrm{d}$ - IVexploration du ««nouum metallum» par un «ternagus»}

Mais revenons au travers-banc d'exhaure. Son importance était considérable, car il assurait à lui seul l'évacuation de l'eau de la totalité $\mathrm{du}$ gisement, ou, plus exactement, de la partie superficielle des deux gîtes métallifères de Vipasca. Il va de soi que ce tra ${ }^{\mathrm{r} e r s}-\mathrm{banc}$ ne pouvait en aucune manière dépendre d'un exploitant en particulier, même pas de ceux qui exploitaient les putei les plus voisins. C'était là en effet une structure d'utilité publique; elle était placée sous le contrôle des services du procura- 
leur (242), qui devait sans doute en affermer l'entretien à une entreprise privée de Vipasca. IJ était en tout cas logique qu'il fût protégé contre toute atteinte. Il faut une découverte comme celle du «nouveau gîte» pour qu'une mesure exceptionnelle permette qu'on y touche.

Quelle est donc cette mesure ? Elle prévoit qu'en vue d'explorer le nouveau gîte un ternagus sera poussé précisément à partir du cuniculus: sa section sera de 16 pieds carrés, soit environ $1,20 \mathrm{mx} \quad 1,20 \mathrm{~m}$. Le problème est de savoir ce que signifie ternagus. On a renoncé à y voir un mot d'origine latine ou grecque et l'on pense que ce mot, comme cuniculus, aurait une origine ibérique. La plupart des commentateuis croient qu'il désigne une galerie, et la précision sur la section rend l'hypothèse vraisemblable. Mais quel serait le but de cette galerie? $\mathrm{Vu}$ la raison pour laquelle son creusement était autorisé, on traduit généralement par «galerie de sondage». Récemment cependant, G. Magueijc a proposé une interprétation tout à fait différente: selon cet auteur (243), un ternagus communiquant avec le cuniculus qui aquam metallis subducet ne peut être lui-même qu'une galerie d'exhaure, mais secondaire, qui unirait au travers-banc principal une nouvelle mine. Nous ne pensons pas que cette interprétation soit la bonne, car elle ne tient pas compte de la nature du gisement: il ne faut pas croire en effet que les mineurs pouvaient, tout le long du travers-banc, trouver plusieurs nouveaux gisements et que nous avons là une mesure organisant l'exploitation à venir de ces derniers. Par ailleurs le mot ternagus apparaît encore, au pluriel, dans Vip. //, 17 et 18; le paragraphe 17 prévoit des sanctions pour celui qui, in ternagis, aurait cherché ou abattu du minerai à moins de quinze pieds du travers-banc d'exhaure: si nous adoptons l'interprétation de Magueijo, cela signifierait que ce qui serait interdit dans la «galerie secondaire d'exhaure» serait autorisé ailleurs, par exemple dans un chantier d'abattage. Quant à Vip. //, 18, il prévoit que l'on ne doit pas pousser de ternagus au-delà des limites du puteus; on se demande alors comment pourrait faire l'exploi-

(2)»2j cf. la formule

procurator ... permi (Vip. 15,2).

(«) Magueijo, p. 136, 152-153.

Conimbriga, 22 (1983), 5-193 
tant qui, voulant unir sa mine au collecteur général (cuniculus qui aquam metallis subducet), creuserait à cet effet une galerie qui ne pourrait pas dépasser les limites de sa concession.

Par conséquent nous en revenons à une interprétation proche de celle qui est généralement admise et nous traduirions ternagus par «galerie de reconnaissance». Dans le cas du paragraphe 15, l'originalité de cette dernière est qu'elle était poussée à partir $\mathrm{du}$ travers-banc d'exhaure, d'où sans doute les limites posées à ses dimensions. Par ailleurs on remarquera que ce sens convient aux emplois de ternagus dans Vip. II, 17 et 18.

\section{$\mathrm{e}$ - Les «putei» à Aljustrel et dans les gîtes pyriteux du Sud-Ouest}

En quoi le procurateur avait-il intérêt à ce que le nouveau gîte fût exploré tout de suite? Pourquoi ne pouvait-il pas attendre que les mineurs d'Aljustrel entreprissent à leurs risques une telle recherche selon la procédure qui, selon Vip. I, 9 et Vip. II, 1 et 2, semble avoir été prévue à cet effet: $Y$ occupatio? C'est que peut-être la façon dont, à Vipasca et dans les autres gisements analogues $\mathrm{du}$ Sud-Ouest, les colons «occupaient» les putei n'était pas aussi libérale qu'on le pense généralement et que le fisc ne laissait pas la zone métallifère livrée à leur bon plaisir. Nous n'avons malheureusement pas de relevé des puits dont l'emplacement était visible en surface en 1873. Nous savons qu'ils étaient nombreux, comme ils l'étaient aussi sur les gisements de même type, par exemple à Concepción (H 6), El Lagunazo (H 9), Tharsis (H 12), Herrerías (H 40), Riotinto (H 43), S. Domingos (POR 7), la Serra de Caveira (POR 36). Nous avons des chiffres plus précis pour le Cabezo de los Silos, à La Zarza (H 19), où l'on comptait plus de huit cents puits jumeaux, pour Sotiel Coronada $(\mathrm{H} 30)$ où on peut dénombrer quelque cent paires de puits jumeaux (fîg. 35), enfin pour Je Cabezo de los Silos - le même nom évocateur qu'à La Zarza à Cabezas del Pasto $(\mathrm{H}$ 30) où ont été inventoriées environ cent quarante-cinq paires de puits, ainsi que quelques puits isolés (fig. 36). Un simple coup d'oeil jeté sur ces deux figures fait regretter que Ton n'ait pas de relevé équivalent pour les autres sites mentionnés ci-dessus et, bien sûr, pour Aljustrel. Mais déjà, à examiner d'un peu près les relevés de surface de Sotiel et 
surtout de Cabezas del Pasto (figs. 35 et 36) publiés il y a un siècle par J. Gonzalo y Tarin, on ne peut manquer de remarquer la disposition régulière des couples de puits (2:4), qui donne irrésistiblement l'impression d'une exploitation systématique, exécutée selon un plan déterminé.

\section{$\mathrm{f}$ - Les conditions de gisement des minéralisations exploitées par les Anciens dans les mines du Sud-Ouest de la Péninsule}

A quoi correspondent donc ces séries régulières de puits? Elles nous paraissent s'appliquer exclusivement à un type de gisement donné, que les Romains ont appris à connaître et à exploiter intensément: les gîtes de pyrite du Sud-Ouest. Nous avons largement traité ailleuis $\left({ }^{245}\right)$ des conditions de gisement de ces minéralisations et nous ne reprenons ci-dessous les traits principaux de cet exposé que pour éclairer les paragraphes ou chapitres de Vip. I et Vip. II traitant de questions proprement minières. Ces gisements ont en général une forme lenticulaire allongée, affectée d'un net pendage. L'essentiel de leur masse minéralisée, à savoir la partie non oxydée, composée de pyrite de fer cuivreuse, n'a pas été exploitée par les Anciens, dont les travaux se situent exclusivement dans la partie superficielle, jusqu'à 80 mètres de profondeur environ, localement jusqu'à 100 ou 120 mètres (246). C'est là la partie du gisement qui a été soumise à l'oxydation et où se sont déroulés des phénomènes d'enrichissement secondaire grâce auxquels le cuivre et les métaux

(244) Gonzalo y Tarín, pl. 30 et 36. Voir ici même les ligures 35 et 36.

Une remarque de Déigny confirme les relevés de J. Gonzalo y TARín.

Cet auteur, qui avait visité les mines du Sud-ouest de la Péninsule avant qu'elles ne fussent défigurées par les travaux modernes, écrivait en 1864: «Les travaux romains étaient faits avec une certaine régularité... L'extraction se faisait par des puits foncés suivant des lignes parallèles et espacés de 25 à 40 mètres les uns des autres. Lorsque les puits devaient avoir une grande profondeur, on faisait des puits jumeaux, afin d'assurer la ventilation». (DELIGNY, Notice, p. 901).

(245) Voir Domergue, Mines, Première partie. Voir aussi ci-dessus, p. $24-30$,

(246) Riotinto (H 43): filon Nord; Aljustrel: Algares (filon du Mur).

Conimbriga, 22 (1983), 5-192 
précieux - or et surtout argent -, contenus à faible teneur dans les sulfures primaires, se sont trouvés concentrés dans des zones préférentielles sous des formes en général clairement reconnaissables et à des teneurs très élevées. Dans la zone de cémentation, située à la base de la zone d'oxydation ou «chapeau de fer», se trouvent de riches sulfures de cuivre secondaires (en particulier, la chalcosite) qui parfois s'enfoncent assez profondément dans la masse pyriteuse non oxydée, tandis que, dans la zone d'oxydation même, sont dispersés des rognons d'oxydes rouges (cuprite) ou noirs (tenorite), eux aussi très riches et de métallurgie facile.

D'autre part, d'après des observations faites principalement à Riotinto $\left(\mathrm{H}\right.$ 43) et à Tharsis $\left(\begin{array}{ll}\mathrm{H} & 12\end{array}\right)$, il $\mathrm{y}$ a entre la zone d'oxydation et la zone de cémentation une étroite couche où se sont concentrés les métaux nobles et particulièrement l'argent. Malheureusement à Aljustrel, le niveau auquel correspond cette zone semble avoir été exploité vers la fin du siècle dernier ou au début de ce siècle, et l'on n'a pas de renseignement sur l'exis tence de cette couche riche en argent. Sans doute le texte même de loi où sont mentionnés les putei argentarii constitue-t-il un puissant argument en faveur de son existence dans le gisement des Algares, et d'ailleurs nous savons sûrement qu'elle est présente à Aljustrel dans le filon aveugle de Moinho (247). Mais nous avons plus haut été conduit à considérer qu'aux Algares, cette couche a pu être moins riche et moins uniforme que dans d'autres gisements comparables $(248)$.

Quoi qu'il en soit, on voit que la partie supérieure de la plupart des gîtes pyriteux était particulièrement riche en cuivre et en métaux nobles. Ces derniers apparaissent localisés presque exclusivement dans une seule couche, en général de faible épaisseur, alors que pour le cuivre, si les sulfures secondaires se situent à la base du chapeau de fer, de riches minerais oxydés sont distribués dans ce dernier sur une bonne hauteur. Si l'on considère que la zone de cémentation (cuivre) et la couche riche en métaux nobles qui l'épouse sont rarement horizontales en raison des anciennes variations du niveau hydrostatique, on comprendra qu'aux yeux des

(247) Voir supra, p. 27 et note 54.

$\left(2 *_{8}\right)$ Voir supra, p. 135. 
mineurs romains, les minéralisations de cuivre et d'argent de la partie supérieure des gisements pyriteux du Sud-Ouest aient pu paraître irrégulièrement distribuées, mais, vu leur richesse, dignes d'être exploitées. Ajoutons enfin que le pendage de la plupart des gîtes permet aux phénomènes d'oxydation de se développer plus largement au toit, ce qui augmente d'autant l'importance des zones susceptibles de renfermer les minéralisations dont nous venons de parler.

\section{$\mathrm{g}$ - Les conditions du découpage des gisements en concessions}

Dans ces conditions, alors que la masse même du gisement est dépourvue d'intérêt pour les mineurs romains - ce qui n'est pas le cas d'un filon classique minéralisé en galène ou en chalcopyrite il existe ici une zone, d'autant plus large que le pendage du gîte est moins accentué, où sont concentrés, de façon irrégulière, de riches minerais de cuivre et des minerais d'argent. Pour les atteindre, il n'est nul besoin de s'enfoncer par l'affleurement jusqu'au coeur du gisement, comme on le faisait dans un filon sulfuré ordinaire; avec une pratique suffisante de ce genre de gîte et connaissant le pendage de la masse minérale, on peut délimiter l'extension de la zone minéralisée intéressante qu'il est facile d'atteindre depuis la surface directement par des puits verticaux, ce qui supprime les difficultés de la circulation dans les descenderles irrégulières ouvertes dans les affleurements mêmes.

Par ailleurs, à Vipasca, les conditions d'exploitation ne sont pas celles qui régnaient par exemple à l'époque républicaine dans les mines filoniennes de la Sierra Morena, où, quand une compagnie fermière ou une entreprise individuelle mettait en valeur un gisement, il est vraisemblable qu'elle l'exploitait tout entier elle-même: elle ménageait simplement les entrées qui lui étaient nécessaires - en général quelques descenderles ou puits ouverts dans l'affleurement. Aussi, dans ces mines filoniennes, jamais nous n'avons vu ces innombrables puits foncés tout au long du chapeau de fer et dans son voisinage du côté du pendage comme c'est le cas dans les gîtes pyriteux du Sud-Ouest. C'est que dans cette dernière région, où, semble-t-il, il faut attendre l'époque impériale pour

\section{Conimbriga, 22 (1983), 5-193}


voir se développer l'activité minière $\left({ }^{249}\right)$, il devait en aller tout autrement : ainsi à Vipasca, le fisc doit compter avec plusieurs petits exploitants (coloni) à qui il faut assurer des revenus suffisants, donc l'exploitation de gîtes rentables, sinon ils quitteront le pays et c'en sera fini de l'exploitation de la mine. D'autre part le fisc s'assurera des revenus d'autant plus importants qu'il y aura davantage d'exploitants. Il ne peut donc laisser à un petit nombre l'exploitation des gîtes - contrairement à ce qui, dans les gisements filoniens de la Sierra Morena, s'est produit à l'époque républicaine et, semble-t-il, continue encore à se produire, au moins localement, sous le Haut Empire $\left({ }^{2} 5^{\circ}\right.$ - — et nous avons vu, d'après les textes de Vip. //, que sa politique reposait à la fois sur l'organisation d'une saine concurrence et sur un développement permanent de l'activité. La nature du gisement et le choix d'un système donné d'exploitation vont donc intervenir dans la façon dont le fisc offrira aux exploitants le gisement dont il a la gestion.

Nous avons constaté plus haut qu'il est possible de délimiter en surface une zone qui est en quelque sorte la projection horizontale — du bas vers le haut — de la zone minéralisée en profondeur. Lorsque, comme à Vipasca dans le cas du nouum metallum du paragraphe 15, le gîte est pratiquement aveugle, on ne peut connaître son extension que pai l'intérieur, d'où la nécessité pour le fisc de la faire explorer, à partir du cuniculus qui a révélé son existence, par le moyen d'un ternagus, foncé dans le gisement sans doute de part et d'autre du travers-banc. $\mathrm{Vu}$ à la fois l'irrégularité et la richesse de la zone minéralisée, l'intérêt du fisc est de multiplier les chances de l'atteindre: d'où l'extraordinaire nombre de puits qui ont été foncés sur les gîtes de ce genre. Mais, comme nous l'avons déjà dit plus haut, il est vraisemblable, à voir les figures 35 et surtout 36, que ces puits n'ont pas été ouverts de façon anarchique. Le rôle du físc a dû être déterminant dans ce domaine et la régularité même de la distribution de ces puits sur un gisement donné nous suggère que la zone délimitée en surface comme zone exploitable était divisée en concessions de superficie uniforme,

(249) Yoi $i_{\mathrm{r}}$ là-dessus Domergue, Mines, Troisième partie.

(250) Cf. Domergue, Mines, Quatrième partie. 
chacune étant destinée à l'ouverture d'un puits de mine - apparemment foncé fréquemment selon la technique des puits jumeaux ( $\left.{ }^{251}\right)$

- et désignée dans Vip. I, 9 par l'appellation puteus locusque putei.

$\mathrm{Ce}$ sont ces concessions que pouvaient «occuper» (202) les colons de Vipasca, selon les dispositions prélmes dans Vip. I, 9 et Vip. II, 1 à 5 , ou qui pouvaient, semble-t-il, leur être assignées, si l'on en croit l'expression... et eos puteos quos occupauerit adsignatosue acceperit que nous allons bientôt trouver dans Vip. II, 18.

A Aljustrel, on a pu ainsi quadriller et diviser en concessions deux bandes parallèles, larges chacune d'une soixantaine de mètres, la plus longue (1100 $\mathrm{m}$ environ) couvrant l'affleurement $\mathrm{du}$ filon $\mathrm{du}$ Mur et débordant largement vers l'est du côté du pendage, l'autre, plus courte $(600$ à $700 \mathrm{~m})$, située à une centaine de mètres à l'est sur le versant, à l'aplomb du filon du Toit (fig. 23). Par ailleurs, en examinant des relevés de chantiers modernes sur lesquels étaient signalés des travaux antiques, nous avons noté chez ces derniers la régularité des intervalles séparant les structures d'accès

- puits, travers-bancs (fig. 28 à 31) -, autre argument en faveur de la division du gisement des Algares en concessions de dimensions uniformes, déjà envisagé sur le terrain.

Ainsi donc, l'étude de Vip. II, 15 l'examen des travaux antiques des Algares et la comparaison avec les relevés de surface des gîtes pyriteux du Sud-ouest anciennement exploités nous ont conduit à la conclusion qu'à Vipasca et sans doute dans les mines de même type, le fisc devait découper le gisement en concessions régulières et proposer - ou, dans certaines circonstances, assignerces dernières aux coloni dans les conditions prévues par la loi. Ceci suppose qu'en cas de découverte d'un nouveau gîte ou d'une extension jusqu'alors inconnue d'un gîte donné, le fisc avait besoin que fût exécutée une reconnaissance préalable, avant de procéder au quadrillage et de livrer aux occupatores les nouvelles concessions. Cette hypothèse d'un découpage régulier des concessions est-elle vérifiable? A l'époque moderne, sur la plupart des sites, l'exploi-

(251) Voir supra, p. 15.

(252) «Usurper», semble-t-il, selon une procédure plus ancienne (cf. Vip. 1,9). 
tation en carrière des masses de pyrite a fait disparaître les vestiges de tous ces puits. A Cabezas del Pasto (H 30) cependant, où aucune masse pyriteuse n'a été localisée au-dessous de la zone où ils ont été creusés (fîg. 36), le site est resté intact: un relevé topographique précis et une prospection de surface extrêmement fouillée permettraient peut-être de juger du bien-fondé de notre hypothèse. Nous n'avons malheureusement pas eu la possibilité de tenter l'expérience.

\section{$\mathrm{h}$ - Les distances à respecter de part et d'autre du travers-banc d'exhaure}

Nous avons déjà commenté le paragraphe 16 dont la deuxième phrase, comme nous l'avons suggéré, a sans doute été ajoutée lors de la dernière rédaction que nous possédons du règlement des mines de Vipasca, et nous avons alors souligné la suite logique qui unissait les paragraphes 14 et 16 , sans que la prescription annexe de Vip. Il, 15, 2 pût l'interrompre. Rappelons donc que tous deux renferment des dispositions concernant la même distance minimale que doivent respecter de part et d'autre du cuniculus les exploitants des puits cuprifères existant dans ce secteur. Vip. II, 14 énonce la règle générale. Vip. II, 16 revient sur cette dernière et précise que l'interdiction vaut même quand il s'agit de recherche ou d'abattage de minerai. Quant à Vip. II, 17 il détermine les peines qui seront infligées aux contrevenants; on retrouve ici la différence qui était déjà apparue aux paragraphes 10 et 13, entre le traitement réservé aux hommes libres et celui que l'on infligeait aux esclaves. Ces derniers recevaient autant de coups de fouet qu'il plaisait au procurateur; ils devaient être vendus par leurs maîtres et être interdits de séjour dans quelque mine que ce soit. Quant aux hommes libres, leurs biens sont confisqués, et ils seront interdits de séjour à perpétuité sur le territoire des mines, sans doute celles de Vipasca. Les ternagi dont il est question dans Vip. II, 17 n'ont, à notre avis, rien à voir avec celui dont il a été question au paragraphe 14. Il s'agit plus vraisemblablement des galeries de reconnaissance poussées par les mineurs (esclaves ou hommes libres) au service d'exploitants dont les concessions sont voisines du cuniculus. 
Le paragraphe 18 est incomplet. Il concerne les exploitations de puits argentifères. Le début de la première phrase est, mot pour mot, la répétition du paragraphe 14 , avec une seule différence: s'agissant de puits argentifères, la distance minimum à respecter de part et d'autre du cuniculus est bien plus grande que pour les puits cuprifères: $18 \mathrm{~m}$ au lieu de $4,5 \mathrm{~m}\left({ }^{253}\right)$. Le reste de la phrase apporte, comme Vip. //, 16, des précisions complémentaires, mais en faisant intervenir des notions nouvelles et un vocabulaire nouveau, qu'il n'est pas facile d'éclaircir. Il est d'abord bien précisé qu'au cours des travaux, il faudra respecter strictement les limites de la concession et ne pas les franchir: les exploitants sont donc tenus d'effectuer les opérations de simple topogiaphie reposant sur la comparaison des mesures faites à l'extérieur et à l'intérieur de la mine. Puis vient une prescription obscure: necue echólas colligito. Tous les commentateurs s'y sont arrêtés, mais aucun n'a donné d'interprétation pleinement satisfaisante. On rapproche généralement le mot du grec غ́кßó $\lambda \eta$, qui, dans un passage de Strabon $\left({ }^{254}\right)$, désigne les stériles abondants produits par les mines d'or voisines d'Abydos. C. Magueijo en tire le sens de «scorie» qui ne convient ici en aucune manière. Schonbauer $\left({ }^{255}\right)$ conserve le sens de «stériles», mais la façon dont il interprète le passage n'est pas très claire: comment aurait-on pu sous terre entasser des stériles dans la zone de sécurité réservée de part et d'autre $\mathrm{du}$ travers-banc si l'on n'y avait pas creusé auparavant des excavations qui pussent les recevoir? Mais en creusant ces dernières on aurait produit d'autres stériles: où les évacuer? Le même problème se posait et il ne semble pas qu'il y ait de solution.

On remarquera cependant que les défenses faites ici concernent le franchissement de limites. Il ne faut pas aller au-delà d'elles: ultra, précise la prescription qui précède celle qui nous intéresse; extra fines putei, dit la suivante. Il est probable que, placée entre les deux, l'expression neue echólas colligito doive concerner

(253) Pour l'absence de travaux au voisinage du travers-banc dit «de la Transtagana», voir supra, p. 26 et fig. 31.

(254) Strabo, 14, 5, 28. C. Conophagos, Le Laurion antique et la technique grecque de la production de Vargent, Athènes, 1980, p. 142-144 (sens de «ecvolades»).

(255) Magueijo, p. 137. Schonbauer, Geschickte, p. 99-100. 
une action pouvant se dérouler hors de la limite des concessions argentifères contiguës à la zone de sécurité; or dans cette expression rien ne semble correspondre aux indications telles que ultra et extra fines putei. Deux solutions nous paraissent alors possibles: ou bien on considère que extra fines putei adsignati complète aussi neue ecbolas colligito et on garde à ecbolae le sens de «stériles» qu'il a chez Strabon. Mais il s'agirait alors de remblais anciens, laissés par les mineurs d'époques plus reculées, qui auraient déjà repéré l'existence de cette couche argentifère et l'auraient exploitée en laissant sur place dans les chantiers mêmes les débris provenant d'un premier tri. A Tharsis $\left(\begin{array}{ll}H & 12\end{array}\right)$ et à Riotinto $\left(\begin{array}{ll}H & 43\end{array}\right)$, cette couche semble bien avoir été exploitée à l'époque tartessienne: pourquoi ne l'aurait-elle pas été à Aljustrel? Dans ce cas les mineurs romains auraient recherché le minerai encore existant dans ces remblais, de la même façon qu'au siècle dernier, dans nombre de mines d'Espagne, on a commencé par traiter les remblais que les mineurs romains avaient laissés dans leurs chantiers.

$\mathrm{Ou}$ bien on considère que l'idée exprimée par ultra dans la prescription précédente est ici contenue dans le préfixe $* e c$ - de ecbolae. Nous reviendrions alors au sens premier du grec $\varepsilon \kappa \beta о \lambda \eta ́$, qui exprime «l'action de jaillir hors de», et, avec le sens de «saillie», s'applique à un rocher ou à une pointe de terre s'avançant dans la mer. Le mot ecbolae ne pourrait-il donc désigner ici le prolongement des poches minéralisées hors des limites de la concession ? Dans cette hypothèse, il aurait été interdit de poursuivre l'abattage (colligere: «recueillir» et, ici, «recueillir en abattant»?) d'un amas minéralisé hors des limites de la concession, à l'intérieur de la zone de sécurité. Autrement dit, pénétrer dans la zone de sécurité même pour un motif justifié comme celui-là était rigoureusement interdit.

Il en va de même pour les galeries de reconnaissance qu'il est interdit de pousser dans la zone de sécurité, à partir des concessions «assignées»... Le reste manque, mais nous compléterions volontiers de la façon suivante: occupatiue, ut cuniculus uioletur. En tout cas, ainsi interprétées, les prescriptions contenues dans la dernière ligne conservée correspondraient exactement, pour les puits argentifères, à celles qui étaient mentionnées aux paragraphes 16 et 17 à propos des puits cuprifères. 


\section{2. «Putei aerarii» et «putei argentarii»}

Nous avons plus haut montré que, dans les gîtes pyriteux du Sud-Ouest auxquels appartient Vipasca, il existe de riches minerais, les uns de cuivres, les autres de métaux nobles et particulièrement d'argent. Ainsi se justifie la distinction établie par Vip. II entre les putei aerarii et les putei argentarii, et celle que fait Vip. /, 7, entre les scauriae argentariae et les scauriae aerariae.

Mais comment et à partir de quel moment une concession pouvait-elle être dite «argentifère» ou «cuprifère»? Avant que le puits ne fût foncé, il semble qu'il ait été difficile de dire quel type de minéralisation il allait rencontrer - argentifère ou cuprifère - ou encore si l'exploitant allait se consacrer à l'exploitation du cuivre plutôt que de l'argent, car, si ce que nous avons dit plus haut est juste, tant l'irrégularité que la richesse des minéralisations faisaient que tout puits foncé dans une concession avait des chances de rencontrer les deux types. Dans cette perspective l'appartenance d'un puits à Tune ou à l'autre des deux catégories dépendait d'abord des minéralisations rencontrées, ensuite du choix de l'exploitant. Il paraît alors difficile qu'on ait pu a priori décider de cette appartenance.

Or, bien que, comme on l'a plus vu haut, le cuivre paraisse avoir été plus commun à Vipasca et l'argent peut-être plus rare ou plus capricieusement distribué $(256)$, il est possible que pour rechercher un minerai plus rare, donc plus cher, surtout lorsque l'Empereur a favorisé cette recherche (257), des mineurs aient pu, délibérément, négliger les minerais de cuivre et soient descendus directement aux niveaux où ils avaient quelques chances de trouver des mineiais d'argent. Dans cette hypothèse, avant même qu'ils aient pu produire du métal, les puits argentifères étaient distincts des puits cuprifères.

Enfin les conditions de gisement respectives des minerais d'argent et des minerais de cuivre peuvent peut-être expliquer pourquoi, en ce concerne les concessions voisines du travers-banc

(256) Yoip ${ }_{s u} p_{\text {raj }}$ p. 135, à propos de Vip. 11,2.

(a57) Cf. Vip. 11,2.

Conimbriga, 22 (1983), 5-193 
d'exhaure, celles qui exploitaient l'argent étaient maintenues à une distance plus grande $(18 \mathrm{~m})$ du travers-banc d'exhaure que celles qui exploitaient les minerais de cuivre $(4,5 \mathrm{~m})$. La couche riche en argent est en effet située à la base du chapeau de fer, donc à un niveau plus proche de celui du travers-banc que les rognons d'oxydes de cuivre dispersés de haut en bas du chapeau de fer. Si elle est abattue systématiquement, les risques d'éboulement qui en résultent sont plus grands — vu le niveau où elle se trouve - que ceux que créerait le nettoyage d'une poche d'oxydes de cuivre bien localisée et située à un niveau plus élevé dans le chapeau de fer. On comprendrait dans ces conditions que la limite des concessions argentifères voisines du travers-banc d'exhaure ait été reculée quatre fois plus loin que celle des concessions cuprifères. Nous ne sommes pourtant pas entièrement satisfait par cette explication, car, au-dessous de la couche de métaux nobles, il y a la zone de cémentation, riche en sulfures de cuivre secondaires dont l'exploitation pouvait être aussi dangereuse à proximité du travers-banc. Mais peut-être était-elle moins régulièrement minéralisée.

\section{Les «putei adsignath}

Le dernier paragraphe de Vip. Il nomme les putei adsignati, dont la loi n'avait pas fait mention jusqu'ici. Quelle est donc cette nouvelle catégorie? Les avis divergent sur ce point: selon les uns, il s'agirait des puits, autrement dit des concessions, achetées au fisc $\mathrm{i}^{258}$ ) et qui, vendues par lui dans les conditions indiquées par Vip. //, 5, auraient eu des limites déjà tracées $\left({ }^{259}\right)$, mais sémantiquement et vu le contexte, rien ne permet de penser qu'un puteus uenditus est un puteus adsignatus. Pour d'autres, ce seraient les puits dont le prix a été payé au fisc $\left({ }^{260}\right)$ ou encore Yadsignatio désignerait une formalité administrative concomitante de $Y$ occupa-

(2 $2 \beta)$ Mis poulet, Tables d'Aljustrel, p. 386; MàgueiJo, p. 137, 154.

$\left({ }^{259}\right)$ Schonbauer, Geschichte, p. 101.

(260) D’Ors, Epigrafía, p. 133. 
tio $(261262263)$. Enfin on a considéré que constituait un mode distinct d'acquisition ( $\left.{ }^{2 \mathrm{e} 2}\right)$, et il semble bien que, si l'on donne au mot adsignatus son sens juridique plein, «assigné», les putei adsignati soient effectivement des puits «assignés» par le fisc à des colons, des puits distincts des putei occupati par conséquent (2e3). Parmi les inscriptions d'Afrique où le mot adsignatus est bien attesté dans ce sens, une $\left({ }^{264}\right)$ est particulièrement intéressante, car elle concerne l'assignation de terres à des colons (coloni par un procurateur de la ratio priuata: dans les deux cas, à Vipasca comme en Mauretania Sitif'ensis,il s'agit de l'assignation à des colons d'une part des domaines impériaux.

Mais que sont donc à Vipasca ces putei adsignati. ${ }^{1}$ Le paragraphe concerne les puits argentifères et il est clair que le sujet des verbes occupauerit et acceperit dans le passage... et eos puteos quos occupauerit adsignatosue acceperit est bien (is) qui puteos argentarios (agety. Les putei adsignati de Vip. 18 sont donc des puits argentifères et cela ne saurait nous étonner dans la mesure où nous pouvons conjecturer d'après Vip. II, 2 qu'à Vipasca les putei argentarii étaient dédaignés par les colons au point que l'Empereur Hadrien dut prendre des dispositions spéciales pour favoriser leur exploitation. Nous avons sans doute affaire ici à une procédure parallèle: parmi les concessions argentifères offertes aux colons, il en est qui, pour des raisons diverses (emplacement jugé peu favorable, etc.), ne trouvaient pas preneur; le fisc, soucieux de faire produire au maximum le domaine minier, assignait alors — sans doute gratuitement, comme paraît l'impliquer la procédure de Vadsignatio - lesdites concessions à des colons, dans des conditions juridiques que nous ignorons. Il nous semble cependant qu'il serait faux de croire que seuls les putei argentarii

(261) D. FLACH, Bergtverksordnung, p. 443.

(262) Cup, Le développement de l'industrie minière, p. 347; KÜBLER, Cuq, p. 421.

(263) Contra, RostovtzefF, Kolonat, p. 358.

${ }^{(264)}$ CIL, VIII, 8812 (déjà cité par CUQ, Le développement de l'industrie minière, p. 347).

Voir aussi $A E, 1946, n^{\circ} 38 ; 1957, n^{\circ} 175 ; 1969-1970, n » 696$, ainsi que $\boldsymbol{T} \boldsymbol{L} \boldsymbol{L}, 980,8$ et 981,3 . 
pussent faire l'objet d'une adsignatio; en droit ce devait être une procédure pouvant toucher tout type de puits, en fait dans Vip. //, 18, les puits concernés étaient des puits argentifères, vraisemblablement pour la raison qu'on vient d'évoquer.

\section{$3^{\circ}$ Rédacteur et destinataire de Vip. II}

Le destinataire de Vip. Il ne peut être que le procurator metalli Vipascensis: le caractère local de nombre des mesures proposées ne laisse aucun doute là-dessus. Et la condition même du personnage - un affranchi impérial - telle qu'on peut l'inférer de sa dénomination même-[.] Vlpius Aelianus - le confirme, comme on peut le constater à la lecture de la liste des procurateurs miniers locaux connus dans la Péninsule Ibérique ${ }^{265}$ ).

Mais qui était le rédacteur? Il est difficile de le dire sans aborder le problème de la nature de cette lex. Cette dernière n'est pas une constitution impériale: si tel était le cas, on aurait en Vip. //, 2 secundum meam lib er alit atem. L'auteur de la lettre n'est donc pas l'Empereur Hadrien. Il est en revanche nécessairement un intermédiaire entre l'Empereur et le procurateur de Vipasca. Mais à quel niveau? S'agit-il du procurator a rationibus, le chef des services palatins du fisc? Examinons donc le caractère de la lex. C'est une mise à jour du règlement organisant l'exploitation des mines de Vipasca, comme le montre l'insertion de la mesure d'Hadrien (Vip. //, 2), et, sans doute aussi, de la prescription autorisant le creusement d'une galerie de reconnaissance dans le nouveau gîte à partir du travers-banc d'exhaure (Vip. //, 15, 2). Que les bureaux palatins fissent connaître au procurateur de Vipasca la faveur d'Hadrien était indispensable, mais il l'était moins qu'ils recopiassent tout le règlement mis à jour. Cette tâche devait revenir plutôt à l'échelon administratif régional, qui, vu le silence des sources sur l'existence d'un possible procurator metallorum Lusitanorum ou Hispanorum ne pouvait être que le bureau

(268) Mroze к, Bergwerksprokuratoren, p. 53 et 54 (7 noms). Voir aussi notre propre liste: Domergue, Mines, Quatrième partie. (12 noms). 
provincial $\mathrm{du}$ fisc dirigé par le procurator prouinciae Lusitaniae. C'est donc lui qui serait responsable de la rédaction du règlement des mines de Vipasca mis à jour et envoyé sous forme de lettre par le procurateur de la province de Lusitanie au procurateur de Vipasca; Vip. II est un fragment de ce règlement.

La lex (266) dont fait partie Vip. II est donc un règlement qui concerne une mine du domaine impérial. De ce fait, comme on l'a déjà montré $\left({ }^{267}\right)$, elle ne peut être qu'une lex dicta, au même titre d'ailleurs que celle à laquelle appartenait Vip. /(268). Mais est-ce la lex metallis dicta mentionnée par Vip. I, 9 ou, puisque Vip. II paraît bien être postérieur à Vip. /, une loi analogue? On reconnaît d'ordinaire à la loi ainsi désignée un caractère général ${ }^{269}$. Dans cette hypothèse, vu le particularisme de nombre de ses dispositions, Vip. II ne peut être cette loi générale. Il est fort probable que certains paragraphes en sont le reflet, mais il ne saurait être question de donner à l'ensemble des prescriptions de Vip. II une portée générale.

\section{Conclusion}

L'étude qui vient d'être faite permet de définir les caractères généraux de la mise en valeur des mines de Vipasca par le fisc et la portée même de Vip. II.

\section{Io Le régime juridique}

a) Les exploitants sont des colons et leur condition est, sur plusieurs points, comparable à celle des coloni des grands domaines impériaux d'Afrique ${ }^{(270}$ ). Comme eux en particulier, ce sont des colons partiaires, puisqu-ils ont la jouissance d'une part du territoire impérial moyennant la remise au fisc de la moitié du minerai qu'ils extraient.

${ }^{(266)} \quad$... ex forma quae hac lege continetur... (Vip. II, ${ }^{2}$ ).

(2ß7) D'ORS, Epigrafía, p. 71.

(2ß8) THIELMANN, p. 63-65.

${ }^{(269)}$ D'ORS, Epigrafía,p. 111; THIELMANN, p. 63.

$\left(27^{\circ}\right)$ Voir en particulier le développement que D. FLACH, Bergwerksordnung, p. 440-445, consacre à ce parallèle. 
Mais il serait vain de vouloir pousser trop loin le parallèle. Par de nombreux aspects leur situation est différente et souvent meilleure que celle des colons africains. Ainsi ils ne dépendent pas de conductores, qui seraient des intermédiaires entre eux-mêmes et le procurateur; s'ils ne disposent pas de gros moyens financiers comme paraisent le montrer les paragraphes concernant la constitution de societates, ils en ont suffisamment pour avoir des esclaves et pour employer des hommes libres; dans la mesure où ils respectent les dispositions légales, ils sont en quelque sorte propriétaires de leur instrument de travail, le puits, puisqu'ils peuvent le vendre ou, dans certaines conditions, en faire donation.

\section{b) Les modes d'acquisition des puits}

Auparavant ils avaient acquis ces puits. Les procédures d'acquisition étaient diverses; notre texte en mentionne quatre. Avant de les énumérer, précisons que le découpage des concessions (puteus locusque putei) était vraisemblablement exécuté au préalable par le fisc. Ainsi délimitées elles étaient proposées aux colons.

1. L' occupatio : c'est une procédure où le colon a l'initiative; parmi les concessions offertes $\left({ }^{271}\right)$, il a le libre choix. Après paiement du pittaciarium (droit de quittance ou d'affichage), il a droit à la jouissance de la concession; mais cette jouissance est d'abord limitée au fonçage d'un puits d'exploitation, qui, juridiquement,

(271) Comme on l'a vu plus haut (cf. p. 103), la procédure de 1 'occupatio suppose des concessions vierges ou considérées comme telles. Contra, RostovTZEFF, Kolonat, p. 356. Or, sans parler des travaux antérieurs, la mine d'Aljustrel a été exploitée dès le début de l'époque impériale (cf. supra, p. 31). Etait-il encore possible, à l'époque de Vip. II, de découper dans le metallum de Vipasca des concessions vierges? D'une part, on peut imaginer que c'est seulement à ce moment là que le système d'exploitation a été rationalisé et que le système du quadrillage préalable a été adopté et appliqué à l'ensemble de la mine sans qu'on ait tenu compte des travaux antérieurs: $\mathrm{du}$ point de vue juridique, toutes les concessions pouvaient donc être considérées comme vierges; d'autre part, la découverte du nouиm metallum a dû provoquer l'extension de l'exploitation vers l'est, et par conséquent le découpage de nouvelles concessions dans cette direction. 
est encore pour l'instant en quelque sorte indivis entre $Y$ occupator qui creuse le puits et le fisc propriétaire du terrain. Une fois atteint le minerai, si Yoccupator paie au fisc une somme fixe, qui représente la valeur de la part qui appartient à ce dernier, il devient propriétaire du puits. Il a donc à ce moment la jouissance totale de la concession qu'il peut exploiter moyennant les conditions que nous rappellerons plus loin.

Pour les putei argentarii dont la mise en valeur semble avoir posé des problèmes à l'époque d'Hadrien, une procédure simplifiée est prévue (Vip. //, 2): les phases préliminaires sont supprimées ainsi que le versement du pittaciarium, et les droits de Yoccupator reviennent au colon qui, le premier, à quelque moment que ce soit, paie au fisc le prix fixe qui représente sa part et qui, ayant été baissé, doit attirer les colons.

Dans Vip. //, 3, 4 et 5 sont envisagés un certain nombre de cas où, après déchéance des exploitants, les droits de Yoccupator reviennent à des tiers.

2. Le vente: le premier cas est celui des puits vendus par le fisc dans les conditions suivantes: un colon n'a pas respecté les conditions juridiques d'exploitation (272) ; le puits tout entier est saisi par le fisc et vendu, sans doute aux enchères.

Mais il est d'autres cas de vente: ainsi les colons peuvent se vendre entre eux, à quelque prix que ce soit, puits et parts de puits, à condition que déclaration en soit faite au fisc.

Dans tous ces cas, les acheteurs paraissent acquérir les droits qui sont ceux de $Y$ occupator (273), mais ils sont soumis aux modalités qui régissent l'exploitation.

3. La donatio: la loi n'en parle qu'à propos de parts de puits (Vip. //, 8, 3), mais si quelqu'un possède toutes les parts d'un puits et en fait donation, c'est bien tout le puits qui fera l'objet de cette donation.

4. L'adsignatio: nous ne connaissons pas les détails de cette procédure, mais on peut penser qu'elle concernait des concessions qui, pour une raison ou pour une autre, étaient dédaignées par les

(272) Voir ci-dessous, p. 132.

(273) Cela nous a paru être impliqué par Vip. //, 5. 
colons et qui, de ce fait, étaient «assignées» par le fisc. Au paragraphe 18, sont considérés comme adsignati seulement des puits argentifères, ce qui paraît dû à des conditions locales particulières qui ont sans doute aussi motivé les mesures de Vip. II, 2.

\section{c) Les modalités juridiques d'exploitation}

A lire Vip. Il, 5, on peut penser qu'une fois acquis un puits et par conséquent la jouissance de la concession dans laquelle il est foncé, les modalités juridiques et administratives d'exploitation sont les mêmes. Du point de vue fiscal, les colons propriétaires d'un puits étaient tenus de livrer au fisc la moitié du minerai produit: en nature ou sous la forme d'une somme d'argent équivalente? Nous pencherions plutôt pour la deuxième solution.

Des peines sévères étaient encourues par les contrevenants. Elles allaient jusqu'à la saisie du puits et à sa mise en vente par le fisc, quand la fraude risquait d'avoir pour conséquence de priver ce dernier de la totalité de ses droits sur la production \{Vip. II, 1). Elles étaient moins fortes quand le fraudeur voulait tricher sur la quantité de minerai effectivement produit $\{$ Vip. Il, 9).

\section{$2^{\circ}$ La position du fisc. La fiscalité}

Représentant l'Etat propriétaire des territoires miniers, le fisc, par l'action du procurateur local, contrôle l'exploitaticn et s'efforce d'accroître la production en vue d'augmenter son propre profit. Il prend des mesures d'incitation \{Vip. II, 2), tient à jour le fichier des colons exploitants \{Vip. II, 8), surveille ces derniers au besoin en suscitant la délation (Vip. II, 1) et sanctionne leurs moindres écarts, tant dans leur comportement vis-à-vis de l'Etat que dans la façon dont ils effectuent les travaux miniers.

Les revenus qu'il tire de l'exploitation elle-même $\left({ }^{274}\right)$ sont de plusieurs sortes:

le prix fixe demandé pour la pars dimidia ad fiscum pertinens dans le cas de facquisition du puits par occupatio. Pour les putei argentarii il s'élève à 4000 sesterces.

(274) A l'exclusion de ceux qu'implique la location des monopoles et autres activités mentionnés dans Vip. 1 . 
le montant du prix atteint par la vente d'un puteus uniuersus.

- le montant des amendes frappant les infractions à la règlementation. Certaines d'entre elles étaient très élevées, comme celle qui punissait la dissimulation de minerai. Par ailleurs le montant de la vente des biens confisqués dans les cas prévus aux paragraphes 10, 13 et 17 devait aller également au fisc.

- enfin et surtout la moitié du minerai produit, sous la forme, à notre avis, de son équivalent en monnaie.

\section{$3^{\circ}$ Aspects techniques}

L'entretien des puits et des chantiers ainsi que certaines dispositions concernant le travers-banc d'exhaure occupent les derniers paragraphes. Ces questions techniques étant rarement commentées au fond, nous nous sommes tout spécialement efforcé de les éclairer, et cela nous a conduit à la constatation que Vip. Il est avant tout un réglement qui concerne exclusivement les mines de Vipasca.

$4^{\circ}$ VIP. II: Un règlement local comprenant des dispositions ayant pu être étendues à Vensemble des gisements de la province métallo génique du Sud-ouest de la Péninsule, et reflétant des principes généraux d'administration.

Nous avons déjà dit plus haut pour quelles raisons Vip. II ne pouvait être la lex metallis dicta mentionnée par Vip. /, 9. Mais cela ne signifie pas qu'on n'y retrouve point l'écho de mesures générales.

On pourrait ainsi distinguer, en allant $\mathrm{du}$ particulier au général :

a) des dispositions purement locales

Il s'agit principalement du paragraphe 15 , dont le commentaire s'enrichit considérablement si on l'examine en se référant aux conditions mêmes de gisement des minéralisations d'Aljustrel. 


\section{b) des dispositions ayant pu avoir une portée régionale}

Il n'est pas fréquent que dans une même mine on puisse exploiter en même temps l'argent et le cuivre. Il y a là une particularité qui a en général échappé aux commentateurs. Elle s'explique si l'on se réfère aux minéralisations que l'on trouve dans la partie supérieure de la plupart des gîtes pyriteux du Sud-Ouest et dont les principales sont celles du cuivre et celles d'argent (275). Par conséquent plusieurs dispositions de Vip. II ont pu être appliquées dans d'autres mines du Sud-ouest. Telle est par exemple la distinction entre les puits argentifères et les puits cuprifères, ou la faveur dont bénéficient, grâce à la libéralité d'Hadrien, ceux qui entreprennent l'exploitation de puits argentifères (Vip. II, 2).

Par ailleurs, les mesures qui concernent les concessions voisines du travers-banc d'exhaure et l'observation d'nne zone de sécurité dont les dimensions de part et d'autre de ce dernier varient selon la nature des puits ont pu être appliquées telles quelles, ou avec des valeurs différentes, dans les autres mines de pyrite du Sud-Ouest de la Péninsule.

Déjà d'ailleurs, dans Vip. /, 7, la mention des scories argentifères et des scories cuprifères trahissait une disposition applicable à nombre de ces dernières.

En revanche, il est peu probable que de telles mesures aient concerné les mines filoniennes de la Sierra Morena. Non que ces dernières, riches en galène argentifère, n'aient pas été parfois pourvues de minerais de cuivre et n'aient pas été exploitées à l'époque romaine $(276)$, mais les minéralisations y sont d'un type différent: ainsi la galène argentifère qu'on $y$ trouve est très abondante et le plus souvent très riche en argent, alors que le cuivre $\mathrm{y}$ est beaucoup plus rare. Les conditions sont donc différentes de celles que suppose Vip. II, où d'ailleurs le plomb

(275) Voir supra, Livre I, p. 24-27, et Livre II, p. 159-161.

(276) $\mathrm{v}_{0}$ ir par exemple la mine de La Loba (province de Cordoue) actuellement en cours de fouille par la RGP 496 CNRS et le Département d'Histoire Ancienne de l'Université Complutense de Madrid, dans le cadre d'une Action Intégrée franco-espagnole (publication prochaine). On consultera aussi Domergue, Mines, Première et Troisième parties. 
n'est même pas nommé alors qu'il le serait abondamment si les mines de la Sierra Morena avaient été visées. Enfin aucune de ces dernières ne présente la distribution régulière de puits qu'on voit à Sotiel Coronada (H 36) ou à Cabezas del Pasto (H 39) (fig. 35 et 36) et qui reflète le découpage des sites en concessions que paraît impliquer l'analyse de Vip. IL

Toutes ces remarques nous font donc conclure que, même si l'on accorde à certaines dispositions de Vip. II une portée régionale, elles n'ont guère pu concerner d'autres mines que celles du SudOuest de la Péninsule.

\section{c) des principes d'administration caractérisant la façon dont le fisc gérait le domaine minier impérial.}

Ces principes paraissent bien, eux, avoir guidé l'administration impériale et avoir eu une portée générale. Ce sont les suivants:

— recours au régime du colonat partiaire.

- «intéressement» des colons aux travaux d'exploitation grâce à des dispositions leur assurant la propriété de leur instrument de travail, le puits, et tendant à leur faciliter la constitution des capitaux par le biais de sociétés.

- variété des modes d'acquisition des puits; ici quatre sont mentionnés: Voccupatio, la uenditio, la donatio, Yadsignatio.

- recherche d'un développement optimum de l'activité minière en vue d'augmenter les revenus du fisc.

- usage de fortes sanctions en cas d'infraction et traitements distincts pour l'homme libre et pour l'esclave.

\section{CONCLUSIONS}

A la fin de chacun des commentaires que nous avons consacrés respectivement à Vip. I et à Vip. //, nous avons rassemblé les données les plus importantes contenues dans ces tables. Nous n'avons pas l'intention de les reprendre ici. Simplement nous voudrions dégager les rapports qui existent entre les deux fragments de lois et les éléments qui permettent de fixer leur date. 


\section{VIP. I et VIP. II: Ressemblances et différences}

On note entre les deux tables plus de ressemblances que de différences véritables. D'une part, toutes deux illustrent ce principe de r«exterritorialité» des domaines miniers impériaux, dont la spécificité a été soulignée encore récemment $\left({ }^{277}\right)$. Mais alors que Vip. I traite principalement de la ferme de certains impôts, offices et services publics - ce qui constitue en quelque sorte l'aspect fiscal de la vie quotidienne à Vipasca - et n'effleure que dans son dernier chapitre les problèmes proprement miniers, Vip. II est tout entière consacrée à l'organisation de l'exploitation des mines.

D'autre part on a voulu différencier Vip. I de Vip. II en faisant de celle-ci un fragment d'une lex metallis dicta générale, à laquelle se réfère Vip. /, 9 ou d'une loi comparable, plus récente. Pourtant, pour le prouver, on ne saurait s'appuyer sur le fait que le bourg de Vipasca n'est pas nommé dans Vip. //, car ce serait oublier que cette table n'est qu'un fragment de loi. Surtout nous avons vu qu'il y a, dans Vip. //, trop de mesures de portée strictement locale ou régionale pour qu'on puisse raisonnablement soutenir cette thèse. Mais il est par ailleurs normal qu'on $y$ trouve aussi le reflet de dispositions plus générales dont certaines sont peut-être inspirées — comme, semble-t-il, dans Vip. /, 9 la procédure de Voccupatio - par la lex metallis dicta en question.

\section{«VIP. I» et «VIP. II»: des mosä̈ques juridiques}

C'est qu'en effet les règlements auxquels appartiennent nos tables sont faits de la juxtaposition de mesures diverses qui, soit pour aligner la situation de Vipasca sur le régime général, soit pour répondre à des besoins locaux, sont insérées parmi celles plus anciennes qui restent en vigueur. Ainsi s'explique l'apparente incohérence qui parfois règne dans la succession des chapitres et des paragraphes: nous avons par exemple souligné dans Vip. Il l'insertion très probable du paragraphe 2 (mesure d'Hadrien en

(277) BurI a , Exterritorialitàt, p. 49-52 et Leges, p. 535-560; D. FlaCH, Bergwerksordnung, p. 440-446. 
faveur des exploitants de puits argentifères) et de la deuxième phrase du paragraphe 15 (creusement d'un ternagus à partir du travers-banc d'exhaure). Par ailleurs telle phrase d'un chapitre de Vip. I nous a paru difficile à comprendre, sans doute en raison d'adjonctions multiples (Vip. I, 3, 2).

Bref ces lois sont de véritables «pots-pourris» de mesures juridiques et fiscales de toutes portées. Les mises à jour successives de ces règlements locaux étaient gravées sur ces tables qu'on fabriquait sur place à peu de frais avec le métal local et dont on se débarrassait lorsque l'insertion d'une mesure récente exigeait une rédaction nouvelle. L'ancienne table, désormais inutile, allait donc au rebut, parmi les scories: c'est effectivement là qu'on a découvert Vip. I et Vip. II.

De tels procédés permettent d'expliquer que, dans nos textes, on trouve des dispositions d'époques différentes. Dans Vip. //, le paragraphe 2 (libéralité d'Hadrien) paraît plus récent que ceux qui l'entourent; de même, le fait que dans Vip. /, P, les deux procédures de Yusurpatio et de Yoccupatio soient juxtaposées, alors que Vip. II ne se réfère qu'à Yoccupatio, fait penser que Vip. Il est plus récente; dans cette hypothèse Vip. /, $\mathrm{P}$ marquerait un stade intermédiaire entre une période ancienne régie par la procédure de $Y$ usurpatio et une époque récente où seule $Y$ occupatio serait en vigueur,

\section{La date de «VIP. /», de «VIP. II» et de la «Lex metallis dicta» citée dans «VIP. /, 9»}

A s'engager dans de telles voies, on est amené à considérer la date de ces deux tables. Le paragraphe 2 de Vip. Il date sûrement de l'époque d'Hadrien (117-138), puisque l'Empereur n'est pas qualifié de diuus, et il est en général considéré comme une adjonction récente dans un texte antérieur. Par ailleurs le fait que, pour compléter Aug., premier mot qui nous est conservé de Vip. //, nous refusions la restitution habituelle [proc.] Aug. nous amène à voir dans cette abréviation la fin d'une dénomination impériale, qu'il n'y a pas de raison de refuser d'attribuer à Hadrien lui-même, vu la fin de la formule qui le désigne au paragraphe suivant: sacratissimi imp. Hadriani Aug. L'ensemble des dispositions fiscales de Vip. //, 
puis la mesure contenue dans le paragraphe 2 peuvent en effet fort bien appartenir à deux moments successifs du règne d'Hadrien. D'ailleurs l'intention que trahissent toutes ces mesures destinées à développer et à organiser l'activité minière est bien la même que celle qu'on décèle par exemple dans la lex Hadriana de rudibus agris dont l'inscription d'Aïn OuasseJ nous fait connaître l'existence ${ }^{(278)}$. Enfin, la deuxième phrase du paragraphe 15, qui semble être aussi une mesure récente (autorisation donnée pour le creusement d'un ternagus à partir du travers-banc d'exhaure, noui metalli explorandi causa), peut également dater $\mathrm{du}$ règne d'Hadrien.

Et Vip. I ? Ses caractères paléographiques ne sont pas différents de ceux de Vip. IL Elle ne saurait donc être beaucoup plus ancienne que cette dernière, même si le contenu de son chapitre 9 fait penser qu'elle est quelque peu antérieure. Aussi peut-on la dater soit de la fin du ler siècle soit du début du Ile, par exemple du règne de Trajan, ou, à la rigueur, des premières années du règne d'Hadrien.

Enfin la référence de Vip. I, 9 à la lex metallis dicta à propos de la procédure de $Y$ occupatio semblerait indiquer que cette loi est récente par rapport à Vip. I. La lex metallis dicta en question pourrait donc dater elle aussi de la fin du ler siècle ou du début $\mathrm{du}$ Ile. Elle serait de peu antérieure à la rédaction du texte de Vip. I qui nous est conservé.

(278) GIRARD-SENN, p. 573-577.

Conimbriga, 22 (1983), 5-193 


\section{BIBLIOGRAPHIE}

Alarcão (J. et A.) - O espólio da necrópole luso-romana de Yaldoca (Aljustrel), dans Conimbriga, 5, 1966, p. 7-109. (= A la RC Ão, O espólio).

Alla N (J. C.). - A mineração em Portugal na antiguidade, dans Boletim de Minas, 2, 1965, p. 11-13 du tiré-à-part (= Allan, A mineração em Portugal).

ANd RE A u (J.). - Les affaires de Monsieur Jucundus, Rome, 1974 (= ANDRE AU, Jucundus).

Ardaillon (E.). - Les mines du Laurion dans l'antiquité, Paris, 1897 (= ARDAILLON).

Arteaga (O.) et Serna (M. R.). - Influjos fenicios en la región del Bajo Segura, dans XIII Congreso Nacional de Arqueología (Huelva, 1973). Saragosse, 1975, p. 737-750 (= ART EAGA-SER A, Influjos).

BaILEY (K. C.). - - The elder Pliny's chapters on chemical subjects, vol. 1, Londres, 1929; vol. 2, Londres, 1932 (= BAILEY, Chemical subjects).

BINDER (J. J.). - Die Bergwerke im romischen Staatshaushalte, dans Z. $f$. Bergbaurecht, 32, 1881, p. 61-94, 191-240 (= BINDER, Bergwerke).

Blanco Freijeiro (A.) et Luzon Nogué (J. M.). - Mineros antiguos españoles, dans A.E.A., 39, 1966, p. 73-88 (= BLanco FreiJeiro-Luzón Nogué, Mineros antiguos).

Bruns (G.). - Lex metalli Vipascensis, dans Z. f. Bechtsgeschichte, 1878, p. $372-383$ (= BRUNs, Lex metalli).

Bruns (G.). - Fontes luris Bomani Antiqui, 1, Leges et negotia, 7e éd. par O. Gradenwitz, Tübingen, 1909 (= B R uns, Fontes).

B u RAT (A.). - Traité du gisement et de l'exploitation des minéraux utiles. Géologie pratique, Paris, 1858 (= Bu Rat, Traité du gisement).

Burat (A.). - Traité du gisement et de l'exploitation des minéraux utiles. Exploitation des mines, Paris, 1859 ( = Burat, Exploitation des mines).

BURIAN (J.). - Einige Bemerkungen über die Exterritorialitàt der hispanischen Bergwerke und der afrikanischen Domànen in der Kaiserzeit, dans Studia antiqua A. Salac septuagenario oblata, Ceskolow, 1955, p. 49-52 (= BURIAN, Exterritorialitàt). 
BURIAN (J.). - Leges metallorum et leges saltuum, dans Z. f. Geschichtswissenschaft, 5, 1957, p. 535-560 (= BuRIAN, Leges).

BURTHE (J.).- - Note sur les tables de bronze d'époque romaine des mines d'Aljustrel (Portugal), dans Annales des Mines, 12e s., 13, 1928, p. 37-39 (= Burthe, Tables de bronze).

Cagnat (R.) - - [sans titre] (séance du 27 juillet 1906), dans CHAI, 1906, p. 328-331 (= CAGNat, CRAI 1906).

CAGNAT (R.). - Un règlement minier sous l'Empire romain, dans $J S, 1906$, p. 441-443 et 671 (=CAGNAT, Règlement).

CÂPANELli (D), - Per un riesame delle leges Metalli Vipascensis, Livourne, $1980,15 \mathrm{p}$.

Crema (L.). - L'architettura romana (Enciclopedia Classica, 3, 12, 1), Turin, 1959 ( = CREMA).

CuQ (E.). —dans CRAI, 1907, p. 95-99 (= CuQ, CRAI 1907).

CuQ (E.). - Un règlement administratif sur l'exploitation des mines au temps d'Hadrien, dans Mélanges Gérardin, 1907, p. 87-133 (= CuQ, Règlement).

CuQ (E.). - Notes d'épigraphie et de papyrologie juridiques, dans NRHD, 32, 1908, p. 311-312 (CUQ, Notes d'épigraphie).

CuQ (E.). - Le développement de l'industrie minière à l'époque d'Hadrien, dans JS, 1911, p. 294-304, 346-356 (= CuQ, Le développement de Vindustrie minière).

Degrass I (A.). - Inscriptiones latinae liberae Rei Publicae. Imagines, Berlin, 1965 (= ILLRP, Imagines).

Deligny (E.). - Notice sur l'origine d'une roue ancienne employée pour l'épuisement des mines et présentée au Conservatoire impérial des Arts et Métiers, dans Compte rendu hebdomadaire des séances, Académie des Sciences, 58, 1864, p. 899-902 (= DELIGNy, Notice).

Demelius (G.). - Z Zur Erklàrung der lex metalli Vipascensis, dans Z. SSt.f. Rechtsgeschichte, Rom. Abt., 4, 1883, p. 33-49 (= Demelius, Lex metalli).

Dess A U (H.). - Inscriptiones latinae selectae, 2ème éd., Berlin, 1954-1955. $(=\boldsymbol{I L S})$.

Domergue (C.). - Les mines de la Péninsule Ibérique à Vépoque romaine (à paraître) (= DoMERGuE, Mines).

Domergue (C.) et Freire de Andrade (R ).- - Sondages 1967 et 1969 à Aljustrel (Poitugal). Note préliminaire, dans Conimbriga, 10, 1971, p. 1-18 (= Domergue-Freire de Andrade, Sondages à Aljustrel).

EsTÁcio Da Veiga (S. P. M.). — A tdbula de bronze de Aljustrel, Lisbonne, 1880. (= EsTÁcio Da VEIGA, A tdbula de bronze).

Estácio da Veiga (S. M. P.). — Antiguidades monumentaes do Algarve, 3, 1889 (= Estácio da Veiga, Antiguidades).

Finiels (P. et Louis (M.). - Les mines romaines d'Aljustrel (Province d'Alentejo, Portugal), dans Cahiers d'histoire ( $t$ d'archéologie, 6, 1936, p. 527-547 ( = F INIE LS-LOUIS, Les mines romaines d'Aljustrel). 
Flach (D.). - Die Bergwerksordnung von Vipasca, dans Chiron, 9, 1979, p. 399-498 (= D. F с ACH, Bergwerksordnung).

FLACH (J.). - La table de bronze d'Aljustrel, dans NRHDFE, 2, 1878, p. 269-282, 645-694 (= J. F LACH, Table de bronze).

Freire de ANDRADE (E.). - Documentos inéditos para a história das minas de Aljustrel no século xix, dans Arquivo de Beja, 23-24, 1966-1967, p. 337-351 (= Freire de Andrade, Documentos inéditos).

Freire de Andrade (R.). - As minas de Aljustrel, dans Boletim de Minas, 4, 1967, p. 73-90 (= Freire de Andrade, As Minas de Aljustrel).

Freire de Andrade (R.). - A lavra romana das minas de Algares e na Herdade do Montinho, dans Actas e memórias do I Congresso Nacional de Arqueologia, 2, Lisbonne, 1970, p. 273-284 (= Freire de Andrade, A lavra romana).

Freire de Andrade (R.). - Jazigo de pirite de Aljustrel. Sua descoberta, situação actual e possibilidades futuras (dactylographié) s.d. (= FREIRE DE ANDRADE, Jazigo).

Girard, (P. F.). - Manuel de droit romain, 7e éd., Paris, 1924 (= Girard, Manuel).

GiraRd (P. F.) et SenN (F.). - Les Lois des Romains, 7e éd. par un groupe de romanistes des «Textes de droit romain», tome I, de P. F. Girard et F. Senn, Università di Camerino, 1977 (= GIRARD-SEN N).

Girau D (Ch.). - La table de bronze d'Aljustiel, dans $J S, 1877$, p. 240-248.

Gonzalo y TARìn (J.). - Descripción fisica, geològica y minerà de la provincia de Huelva, 2, Madrid, 1888 (-Gonzalo y Ta Rìn, Huelva).

Gosse (G.). - Las minas y el arte minerò de Espana en la antigiiedad, dans Ampurias, 4, 1942, p. 43-68 (= Goss É, Minas).

Hayes (J. W.). - Late Roman Pottery, Londres, 1972.

HEALY (J. F.). - Mining and metallurgy in the greek and roman world, Londres, 1978.

HirSCHFE LD (O.). - Die kaiserlichen Verwaltungsbeamten bis auf Diocletian, 2, Berlin, 1905 (= Hi RSCHFELD, Verwaltungsbeamten).

HÜ BNER (E.). - Lex metalli Vipascensis, dans Ephemeris Epigraphica, 3, 1877, p. 165-187 (= Hü BNER, Lex metalli).

Hübner (E.). - Metallum Yipascense, CIL, II, Berlin, 1892, p. 788-801 (= HÜBNER, Metallum).

KNIEP (F.). - Argentaria Stipulatio, dans Festgabe des Juristischen Fakultät Jena für August Thon, Jena, 1911, p. 1-62 (= KNIE P).

Kolendo (J.). - Le colonat en Afrique sous le Haut-Empire, Paris, 1976.

Koschaker (P.). - Besprechung zu J. B. Mispoulet, Le régime des mines à Tépoque romaine et au Moyen Age d'après les tables d'Aljustrel, dans Z. SSt.f. Rechtsgeschichte, Röm. Abt., 30, 1909, p. 462-465.

KRETZSCH MER (F.). - La technique romaine, Bruxelles, 1966 (= KretzSCHMER).

KÜBLER (B.). - Besprechung zu E. CuQ, Un règlement administratif sur Texploitation des mines au temps d'Hadrien, dans Z. SSt. f. Rechtsgeschichte, Röm. Abt., 30, 1909, p. 420-422 [= Kü B LE R, Cuq). 
Kübler (B.). - Besprechung zu E. Schonbauer, «Beitrage zur Geschichte des Bergbaurechts», dans Z. Sst. f. Rechtsgeschichte, Rom. Abt., 49, 1929, p. 569-575 (= KÜB LER).

Lambrino (S.). - Catalogue des inscriptions latines du Musée Leite de Vasconcelos, dans O Arqueólogo Português, 8e s., 1, 1967 (= Lambino, Catalogue).

La Roca (E.) De Vos (M. et A.), Coarelli (F.). - Guida archeologica di Pompei, Rome, 1976 (= LA Roca et alii).

LEFORT (J.). - La table de bronze d'Aljustrel, d'après M. Soromenho, dans Revue générale du Droit, de la: Législation et de la Jurisprudence en France et à Vétranger, 1, 1877, p. 297-299.

Leitão (J. M.). - Relatorio annual da Inspecção Geral das Minas, 1860-1861, dans Roletim do Ministério das Obras Públicas, Comércio e Indústria, 6, 1862, p. 476 (= LEIT Ã O, Relatório).

Louis (H.). - Some aspects of mining laws under the Roman Empire, dans Proceedings of the University of Durham Philosophical Society, 6, 1920-1921, p. 32-46 (— Louis, Mining Laws).

Luzon (J. M.). - Instrumentos mineros de la España antigua, dans La minería hispana e iberoamericana, León, 1970, p. 221-258 (= Luzon, Instrumentos mineros).

Magueijo (C.). - A lex metallis dicta (117-138 d.C.), dans O Arqueólogo Portugués, sér. 3, 3, 1969, p. 125-163 (= MagueIJo).

Mispoulet (J. B.). - La lex metallis dicta récemment découverte en Portugal, dans Revue générale du Droit, de la Législation et de la Jurisprudence en France et à Vétranger, 31, 1907, p. 20-32 (= Mispoule T, Lex metallis).

Mispoulet (J. B.). - Le régime des mines à l'époque romaine et au Moyen Age d'après les tables d'Aljustrel, dans NRHD, 31, 1907, p. 345-391; 491-537 (= Mispoule T, Tables d'Alj ustrel).

Mispoulet (J. B.). - Le régime des mines à Vépoque romaine et au Moyen Age d'après les tables d'Aljustrel, 124 p. (texte de l'article précédent, accompagné d'additions très importantes), 1908 (= Mispoulet, Régime des mines).

Mommsen (Тн.). - Die pompeianischen Quittungstafeln des L. Caecilius Jucundus, dans Hermes, 12, 1877, p. 88-141 (spécialement p. 100-102) (= Mommsen, Quittungstafeln).

Monteiro (S.) et Barata (J. A.). - Exposição industrial portuguesa. Catálogo descriptivo da Seç̧ão de minas, Lisbonne, 1889, p. 95-104 (MonTEIRO-BARATA, Exposição).

MrozeK (S.). - Les conditions de travail dans les mines romaines au II siècle de n.è. Vi pasca-Alburnus Maior, dans Roczniki Dziejow Spolecznych i Gospodarczych, 36, 1964, p. 28-30 (= Mrozeк, Travail).

MrozeK (S.). - Die kaiserlichen Bergwerksprokuratoren in der Zeit des Prinzipats, dans Zeszyty Naukowf, Nauki Humanistyczno-Spoleczne, 32, 1968, Historia IV, p. 45-67 (= Mroze к, Rergwerksprokuratoren). 
Mroze K (S.). - Aspects sociaux et administratifs des mines d'or en Dacie, dans Apulum, 1968, p. 307-326 (= Mroze к, Mines d'or).

NeUBuRg (C.). - Das neugefundene Bruchstück eines romischen Berggesetzes, dans Z.f. d. gesamte Staatswissenschaft, 63, 1903, p. 367-391.

Ors (A. d'). - Sobre la lex metalli Vipascensis II, dans Jura, 2, 1951,

p. 127-133 (= D'Ors, Lex metalli).

Ors (A. d'). - Пıтє́́кıоv - pittaciarium, dans Aegyptus, 31, 1951, p. 339-343 ( - D'Ors, Pittaciarium).

Ors (A. d'). - Epigrafía jurídica de la España romana, Madrid, 1953, p. 71-133 (= D'Ors, Epigrafia).

Pinedo Vara (I.). - Piritas de Huelva. Su historia, minería y aprovechamiento, Madrid, 1963 (PInedo Vara, Piritas).

RaMin (J.). - La technique minière et métallurgique des Anciens (Coll. Latomus, 153) Bruxelles, 1977.

RE (C.). - La tavola Vipascense, dans Archivio Giuridico, 23, 1879, p. 327$-388(\mathrm{RE})$.

ribeiro (C.), Carneiro de andrade (E.), Aguiar (A. A. de). Relatorio sobre as minas de pyrite de ferro cúprico das cercanias de vila de Aljustrel, Lisbonne, 1873 (= RI BEIRO et alii, Relatorio).

Riccobono(S.), baviera (G.), Ferrini (C.), Furlini (G.), Arangio-ruiz (V.). - Fontes Iuris Romani Anteiustiniani, I-III, Florence, 1940-1943 (=FIRA, I-III).

Rodríguez De BerLanga (M.). - Los bronces de Lascuta, Bonanza y Aljustrel, Málaga, 1881, p. 623-830 (= Rodríguez DE BERLANGA).

RostovtzefF (M.). - Geschichte der Staatspacht in der romischen Kaiserzeit, dans Philologus, 9, 1904, p. 329-512.

RostovtzefF (M.) - Sludien zur Geschichte des romischen Kolonates, Leipzig-Berlin, 1910 (= RostovtzefF, Kolonat).

SALKIELD (L. U.). - Ancient slags in the South-West of the Iberian Peninsula, dans La minería hispana e iberoamericana, Léon, 1970, p. 85-98 ( = SALKIELD, Ancient slags).

SchónbaueR (E). - - Das Bergrecht von Vipasca, dans Bergrechtliche Rlàtter. Beilage zur Oesterr. Z. f. Berg-und Hüttenwesen, 8, Heft 3, p. 125-137 (republié dans Labeo, 15, 1969, p. 327-336).

Schonbauer (E.). - Zur Erklarung der lex metalli Vipascensis, dans Z. d. SSt. f. Rechtsgeschichte, Rom. Abt., 45, 1925, p. 352-390 et 46, 1926, p. 181-215 (= SCHONBAUER, Lex metalli).

Schonbauer (E.). - Beitràge zur Geschichte des Bergbaurtchts, Munich, 1929 (= Schonbauer, Geschichte).

Schubart $(\mathrm{H} \cdot) \cdot-$ - Die Kultur der Rronzezeit im Südwesten des Iberischen Halbinsel, Berlin, 1975 ( = Sc Hu B A R T, Die Kultur).

SCiAloja. - Nota ad un punto controverso della lex metalli Vipascensis, dans Archivio Giuridico, 24, 1880, p. 482-484 (Scial oJ A, Lex metalli).

SеEск (O.). - Colonatus, dans RE, 4, 1901, col. 484-495 ( = SEеск, Colonatus). 
Soromenho (A.). - La table de bronze d'Aljustrel, Lisbonne, 1876 ( = SoroMENHO).

Spinder (K.), Castelo branco (A. de), Zbyszewsit (G.), Yeiga ferreira (O. da). - Le monument à coupole de l'âge du bronze final de la Roca do Casal do Meio (Calhariz), dans Comunicações dos Serviços Geológicos de Portugal, 57, 1973-1977, p. 91-153 (= SPINDLER et alii, Le monument).

Та̃ с к о ом (U.). - Studien iiber den Bergbau der rômischen Kaiserzeit, Uppsala, 1937 (= Та̃ СКОцм, Bergbau).

Talamanca (M.). - Contributi alio studio delle vendite all'asta nel mondo classico, dans Atti della Accadentia Nazionale dei Lincei. Memorie. Clause di Scienze morali, storiche e filologiche, ser. 8, vol. 6, fase. 2, Rome, 1954 (= TALAMANCA).

Thielmann (G.). - Die romische Privatauktion zugleich ein Beitrag zum rômischen Bankierrecht (Berliner Juristische Abhandlungen, 4), Berlin, 1961 (= THIELMANN).

Veiga Ferreira (O. da) et Freire de Andrade (R.) - Algumas marcas de oleiro em terra sigillata de Vipasca (Aljustrel), dans Bevista de Guimarães, 74, 1964, p. 1-6 (= Veiga Ferreira-Freire de ANdrade, Marcas).

VendeUVRE (J.) - Contribution à Vétude du régime minier romain. Etude sur la table d'Aljustrel découverte en 1906, Dijon, 1910.

Vernhet (A.). - Notes sur la terre sigillée de la Graufesenque, Millau, 1975 (dactylographié) ( = VernheT, Notes).

Vian a (A.), freire de Andrade (R.), Veiga ferreira (O. da). - Minerações romanas de Aljustrel, dans Comunicações dos Serviços Geológicos de Portugal, 25, 1954, p. 79-92 (= VIana et alii, Minerações romanas).

Voelkel (K.)-Die beiden Erztafeln von Vipasca und das deutsche Bergrecht, dans Z. f. Bergrecht, 55, 1914, p. 182-243 (= Voelkel, Vipasca).

Wickert (L.). —Bericht iiber eine zweite Beise zur Vorbereitung von C.I.L. II, Suppl. 2, Berlin, 1931, p. 9-12 (=WICKERT, Bericht).

WiLmanNs (G.).--Die romische Bergwerksordnung von Vipasca, dans Z. $f$. Bergrecht, 19, 1878, p. 217-232 (= WilmanNs).

Zycha (A.). - Besprechung zu E. SChonbauer, Beitrage zur Geschichte des Bergbaurechts, dans Z. S St. f. Bechts geschichte, Boni. Abt., 50, 1930, p. 407-423 (= ZYCHA). 
TRADUCTIONS DE VIPASCA I ET DE VIPASCA II

\section{PASCA I}

En allemand:

WiLMANNS (G.). - Die romische Bergwerksordnung von Vipasca, dans Z. $f$. Bergrecht, 19, 1878, p. 219-230.

SchonbaUeR (F). - Das Bergrecht von Vipasca, dans Bergrechtliche Blatter. Beilage zur Oeslerr. Z. f. Berg-und Hütlenwesen 8, Heft 3, 1913, p. 125-137 (= Labeo, 15, 1969, p. 327-336).

Thielmann (G.). - Die romische Privatauktion zugleich ein Beitrag zum romischen Bankierrechl (Berliner Juristische Abhandlnngen, 4, Berlin 1961, p. 267-272.

FlaCH (D.). - Die Bergwerksordnung von Vipasca, dans Chiron, 9, 1979, p. 407-413.

En anglais:

Nostrand (J. J. van). - Roman Spain, dans An Economie survey of Ancient Borne, publié par T. Frank, 3, Baltimore, 1937, p. 167-171.

Lewis (N.) et Reinhold (M.). - Boman Civilization, 2, New-York, 1955, p. 191-194.

Cummings (A. D.), Chalmers (W. R.), mattingly (H. B.). - A roman mining document, dans Mine and Quarry Engineering, 22, 1956, p. 341342.

Johnson (A. Ch.), Coleman norton (P. R.), Bourne (F. G.).-Ancient roman statutes, Austin, 1961, p. 163-165, nº 206.

En espagnol:

Rodríguez De B erlang a (M.). - Los bronces de Lascuta, Bonanza y Aljustrel, Málaga, 1881, p. 629-638.

En français:

Flach (J.). - La table de bronze d'Aljustrel, dans NBHDFE, 2, 1878, p. 689-694.

Conimbriga, 22 (1983), 5-193 


\section{PASCA II}

En allentanti:

VoelKel (K.). - Die beiden Erztafeln von Yipasca und das deutsche Bergrecht, dans Z.f. Bergrecht, 55, 1914, p. 192-218.

Schönbauer (E).--Das Bergrecht von Vipasca, dans Bergrech liehe Blätter. Beilage zur Oesterrr. Z. f. Berg-und Hüttenwesen, 8, Heft 3, 1913, p. 137-149 (=Labeo, 15,1969 , p. 337-345).

Schön bauer (E.). - Beiträge zur Geschichte des Bergbaurechts, Munich, 1929, p. 104-107.

Thielmann (G.). - Die römische Privatauktion zugleich ein Beitrag zum römischen Bankierrecht, (Berliner Juristische Abhandlungen, 4), Berlin, 1961, p. 272-275.

Flach (D.). - Die Bergwerksordnung von Vipasca, dans Chiron, 9, 1979, p. 403-407.

En anglais:

Nostrand (J. J. van). - Roman Spain, dans An Economic Survey of Ancient Borne, publié par T. Frank, 3, Baltimore, 1937, p. 171-173.

Lewis (N.) et Reinhold (M.). - Boman Civilization, 2, New-York, 1955, p. 188-191.

Cummings (A. D.), Chalmers (W. R.), mattingly (H. B.) .- A roman mining document, dans Mine and Quarry Engineering, 22, 1956, p. 339340.

Johnson (A. Gh.), Coleman Norton (P. R.), Bourne (F. C.).- Ancient roman statutes, Austin, 1961, p. 177-178, $\mathrm{n}^{\circ} 233$.

En français:

Cagnat (R.). - CBAI, 1906, p. 329-331.

Mispoulet (J. B.). - Le régime des mines à l'époque romaine et au Moyen Age d'après les tables d'Aljustrel, dans NBHD, 31, 1907, p. 356-385.

CHARLES-PICARD (G.) et Rouge (J.). - Textes et documents relatifs à la vie économique et sociale dans l'Empire romain (31 avant J.-C. - 225 après J.-C.), Paris, 1969, p. 179-181 (traduction des § 2-13).

En portugais:

Magueijo (C.).-A lex metallis dicta (117-138 d.C.), dans O Arqueólogo Português, 3e s., 3, 1969, p. 132-137.

Conimbriga, 22 (1983), 5-193 


\section{LEGENDE DES FIGURES}

1 - Mine d'Aljustrel: le gisement des Algares vu du nord-est (cliché pris du sommet de la colline de Malpique). Au second plan, le chapeau de fer du fdon du Mur est très clairement visible entre le puits Viana dont on distingue le chevalement vers la gauche et les bâtiments administratifs à droite. Au-dessous de ces derniers, au pied du talus, passait le chapeau de fer du filon Est (ou filon du Toit), avant qu'il ne disparût (cf. fig. 3 et 4). Entre les eucalyptus au premier plan et le chapeau de fer du filon du Mur, des déblais modernes recouvrent une zone où devaient déboucher un grand nombre de puits antiques.

Le sommet du chapeau du fer du filon du Mur est surmonté d'un petit château d'eau, près duquel se trouvent les restes de constructions $\mathrm{du}$ ier et du IIle $\mathrm{s}$. de notre ère; là aussi fut découvert le fragment de vase campaniforme. Au-delà, s'étendaient la nécropole de Valdoca et l'agglomération minière de Vipasca. A l'arrière-plan, les plateaux de l'Alentejo.

2- Mine d'Aljustrel, gisement des Algares: coupe transversale dans le chapeau de fer du filon du Mur, près du puits Viana. L'excavation visible au centre du cliché est sans doute d'origine antique, mais a dû être agrandie à l'époque moderne.

3 - Mine d'Aljustrel, gisement des Algares: sur la quasi-totalité de son tracé, le chapeau de fer du filon Est a aujourd'hui disparu: à son emplacement on ne voit plus qu'une tranchée presque entièrement colmatée, comme ici, au pied des bâtiments administratifs, au sud du chemin qui conduit à ces derniers.

4 - Mine d'Aljustrel, les Algares: dans la tranchée représentée à la figure 3, un petit entonnoir signale l'existence d'un puits antique sous-jacent, colmaté à l'époque moderne. 
5 - Mine d'Aljustrel, les Algares: section transversale dans la partie septentrionale, seule conservée, du chapeau de fer du filon Est, au nord du chemin conduisant aux bâtiments administratifs. Sur le cliché, le «gossan» coloré de brun par les oxydes de fer est visible dans toute son épaisseur, de gauche à droite. On remarquera que son affleurement ne se signale par aucun relief particulier. Les déblais visibles en haut à droite sont modernes.

6 - Mine d'Aljustrel, les Algares: le chapeau de fer du filon Est, de part et d'autre de la tranchée sur la paroi de laquelle il est parfaitement visible en coupe (voir aussi la fig. 5). En revanche, comme le montre clairement le premier plan, son affleurement — quoique à peine couvert de débris de schistes bruns - est pratiquement indécelable.

7 - Mine d'Aljustrel, les Algares: l'entrée du travers-banc dit «de la Transtagana», mais en fait d'origine antique. Etat actuel (photographie prise en mars 1980).

8 - Mine d'Aljustrel, les Algares: puits antique rempli de déblais, visible dans une coupe transversale du chapeau de fer du filon du Mur.

9 - Mine d'Aljustrel, les Algares: monceaux de scories antiques recouvrant un mur arasé (en bas, à gauche), au voisinage du Cabeço do Azinhal.

10 - Mine d'Aljustrel: le versant oriental des Algares, entre l'entrée du travers-banc dit «de la Transtagana» (non visible sur le cliché, mais située à gauche et vers le bas) et le chapeau de fer du filon du Mur (en haut). Le sol est bouleversé et couvert de déblais de toutes époques, si bien que les orifices des puits de jour du travers-banc dit «de la Transtagana» et des puits d'exploitation antiques sont depuis longtemps invisibles.

11-Mine d'Aljustrel, les Algares: puits antique de section rectangulaire $(1,20 \mathrm{~m} \times 0,90 \mathrm{~m})$ foncé au toit du filon Est. Sur la paroi, on distingue des encoches superposées, espacées de $0,30 \mathrm{~m}$ à $0,40 \mathrm{~m}$, destinées à faciliter la remontée.

12 - Mine d'Aljustrel, les Algares: galerie antique voûtée, de petite section (h.: $0,90 \mathrm{~m} ; 1 .: 0,80 \mathrm{~m}$ ) poussée à partir du puits représenté à la figure 11. Des niches creusées dans les parois étaient destinées à recevoir des lampes.

13-Mine d'Aljustrel: échelle à six encoches, constituée par un tronc de chêne vert; rigole, également en chêne vert (Museu Nacional de Arqueologia e Etnologia, Belém). 
14 - Mine d'Aljustrel: deux poulies en chêne vert (Museu Nacional de Arqueologia e Etnologia, Belém).

15 - Mine d'Aljustrel: vase en bronze, à lèvre plate (Museu Nacional de Arqueologia e Etnologia, Belém).

16 - Mine d'Aljustrel: écope en chêne vert pourvue d'un manche, d'une seule pièce; racloir, également en chêne vert (Musée de la mine d'Aljustrel).

17-Mine d'Aljustrel: plaque en bronze trouvée en 1876 parmi les scories antiques et sur laquelle sont gravés plusieurs chapitres d'un règlement (Vip. I) d'époque impériale, face 1 (Musée des Serviços Geológicos de Portugal; photo du Musée).

18 - Mine d'Aljustrel: la plaque en bronze trouvée en 1876, face 2 (Musée des Serviços Geológicos de Portugal; photo du Musée).

19 - Mine d'Aljustrel: la plaque en bronze découverte parmi les scories romaines en 1906, et qui porte le fragment de loi appelé Vip. II (Museu Nacional de Arqueologia e Etnologia, Belém; photo du Musée).

20- Vip. /, face 1: fac-similé du texte publié par HüBner, CIL, II, Supplt., 1892, p. 789. Les corrections mineures, signalées supra, livre II, p. 42-45, ont été ajoutées.

21 - Vip. 1, face 2: fac-similé du texte publié par HüBner, CIL, II, Supplt., 1892, p. 790. Il comporte les quelques corrections indiquées supra, livre II, p. 42-45.

22- Vip. /, face 1: coin inférieur gauche. Calque de frottis, montrant les incisions qui entaillent la plaque et les traces des chocs qu'elle a subis. Remarquer en particulier, sous la dernière ligne du texte, les trois incisions parallèles que l'on a longtemps considérées comme représentant le chiffre III.

23 - Mine d'Aljustrel, gisement des Algares. Haut: le site. Les deux chapeaux de fer et les vestiges antiques: le travers-banc (dit «de la Transtagana») et ses puits de jour (d'après le plan ${ }_{1} \mathrm{~B} / 2$, fig. 25), les aires couvertes de scories (d'après le plan de 1867, fig. 24), la nécropole, l'emplacement très probable du bourg de Vipasca. Le puits Yiana est moderne.

Bas: section longitudinale dans le plan du travers-banc, avec indication des filons et des chapeaux de fer surmontant les masses de pyrite non oxydée. A gauche, sont signalés les niveaux.

Pour la légende, voir les figures 24 et 25. 
24 - Mine d'Aljustrel, les Algares: plan du 30 septembre 1867, d'après la copie du 5 janvier 1901 (calque C. Domergue). On remarquera que le chapeau de fer du filon du Toit n'est pas signalé. En revanche, à l'est du moulin Caiado, un tronçon de travers-banc jalonné de 3 puits est marqué A (vraisemblablement «antigo»).

Légende: 1 : scories. 2 : scories tachées de vert. 3 : zone cuivreuse. 4: chapeau de fer affleurant. 5: puits. 6: moulin à vent. 7: entrée de travers-banc. 8 : chemin.

25 - Mine d'Aljustrel, les Algares: plan ${ }_{1} \mathrm{~B}_{/ 2}$ (calque C. Domergue).

Sur l'original, le tracé du chapeau de fer du filon du Toit a été porté au crayon bleu, postérieurement à l'exécution du plan; il en va de même pour la tranchée de sortie du travers-banc, dessinée au crayon gris. A noter l'alignement des puits de jour qui jalonnent le travers-banc d'exhaure dit «de la Transtagana»; ce dernier coupe le filon de Toit, avant d'atteindre le filon du Mur.

Légende: 9: chapeau de fer du filon du Toit. 10: puits moderne (pour les autres symboles, voir fig. 24).

26- Mine d'Aljustrel: les travaux antiques des Algares, d'après un relevé effectué par Gérard et Yolpelière et publié par E. CuQ, Le développement de Vindustrie minière, p. 352-353. Sont représentés, selon toute vraisemblance, le filon du Mur (en bas) et le filon Est (en haut), d'ailleurs plus proches l'un de l'autre qu'ils ne sont dans la réalité.

Les chiffres en italique ont été ajoutés par nous.

Légende: 1: travaux anciens. 2: puits anciens rencontrés au niveau 60. 3: puits anciens rencontrés au niveau 40.

27 - Mine d'Aljustrel: détail de la figure 26 (d'après Cuo, Le développement de Vindustrie minière, p. 354). Même légende qu'à la figure 26.

28 - Mine d'Aljustrel. Les Algares, filon du Mur. Niveau 40, 34e pilier, 1ère tranche (d'après un relevé de chantier, Arch. Mines d'Aljustrel, dossier 1, 1903). En noir, les travaux antiques (3 puits et un travers-banc); en tracé normal, les travaux modernes: puits $(\mathbf{j} \backslash \mathbf{j})$, galeries, traverses et chantiers d'abattage. Hachuré: veines minéralisées riches en cuivre (jusqu'à $5 \% \mathrm{Cu}$ ) ; hachuré et pointillé: haute minéralisation en cuivre (de 5 à $14 \% \mathrm{Cu}$ ). De part et d'autre, le minerai est plus pauvre (teneur en $\mathrm{Cu}$ souvent inférieure à $1 \%$ ).

La direction générale des veines est N 150 à $168^{\circ}$; le pendage est de $84^{\circ} \mathrm{E}$.

29 - Mine d'Aljustrel, Les Algares, filon du Mur. Niveau 60, 34e pilier, se tranche (d'après un relevé de chantier, Arch. Mines d'Aljustrel, dossier 2, 1903). On reconnaît les puits ${ }_{1}$ et 2 de la figure 28. 
30 - Mine d'Aljustrel. Les Algares, íilon du Toit (ou filon Est). Niveau 60,

$25 \mathrm{e}$ pilier, $7 \mathrm{e}$ tranche (d'après un relevé de chantier, Arch. Mmes d'Aljustrel, dossier 3, 1908). On remarquera la régularité des intervalles entre les structures antiques (1, 2, 4: puits; 3: tronçon de travers-banc).

La direction générale de la minéralisation est $\mathrm{N} 168$ à $171^{\circ}$.

31 - Mine d'Aljustrel. Les Algares, filon du Toit (ou filon Est). Niveau 60, 27e pilier, 1ère tranche (d'après un relevé de chantier, Arch. Mines d'Aljustrel, dossier 3, 1908-1909). Un tronçon du travers-banc dit «de la Transtagana» (en fait le travers-banc d'exhaure antique, élargi en 1876 par la compagnie ainsi nommée) est signalé à l'ouest du filon du Toit, entre ce dernier et le filon du Mur.

32 - Mine d'Aljustrel. Les Algares, filon du Toit (ou filon Est). Niveau 60, 32e pilier, 5e tranche (d'après un relevé de chantier, Arch. Mines d'Aljustrel, dossier 3, 1908-1909). Les deux travers-bancs antiques sont parallèles et distants de $15 \mathrm{~m}$ l'un de l'autre.

33 - Mine d'Aljustrel: puits intérieur de $8 \mathrm{~m}$ de profondeur, découvert en 1921 au niveau 112 de la mine des Algares (filon du Centre), à proximité (6 $\mathrm{m}$ environ) de la masse minéralisée. Ce puits était équipé de quatre treuils (les dessins ci-dessus sont les fac-similés des figures 1 à 5 illustrant l'article de VIAN A et alii, Minerações romanas),

$a$ - Le puits (1 $\mathrm{m} \times \mathbf{1} \mathrm{m})$ boisé, avec ses treuils (1) et les couffins pendant au bout du câble.

$h$ - Plan de l'orifice du puits.

$c$ - Détail d'une poulie et du système de fixation des axes (3) à l'aide de coins ( $\mathbf{1}$ et $\mathbf{2}$ ).

$d$ - Détail de l'installation du guide-câble (1 et 2) et du tambour muni de ses cabestans à quatre bras (3).

La figure $e$ présente une coupe verticale dans un chantier d'abattage où sont utilisées des échelles faites de troncs d'arbres munis d'encoches (ef. fig. 13). A: filon. B: traverse. C: puits. D: chantier d'abattage.

34 - Mine d'Aljustrel, matériel céramique. Provenance: Mangancha ( ${ }^{\text {os }} 1$ et 2), scories des Algares ( $\mathrm{n}^{\text {os }} 3$ et 4), «Maison du Procurateur» $\left(n^{\text {os }} 5\right.$ et 6$)$.

35- -Mine de Sotiel Coronada (H 36): relevé des vieux travaux superficiels et du tracé des travers-bancs antiques (d'après GONZALO Y TARIN, Huelva, pl. 30). On notera en particulier la densité des puits jumeaux (deux points noirs juxtaposés) et le nombre des travers-bancs (double tireté).

36- Mine de Cabezas del Pasto (H 39): relevé des vieux travaux superficiels d'après GonZalo Y TARIN, Huelva, pl. 36. A remarquer la disposition régulière et les alignements de puits jumeaux.

Conimbriga, 22 (1983), 5-193 
(Página deixada propositadamente em branco) 


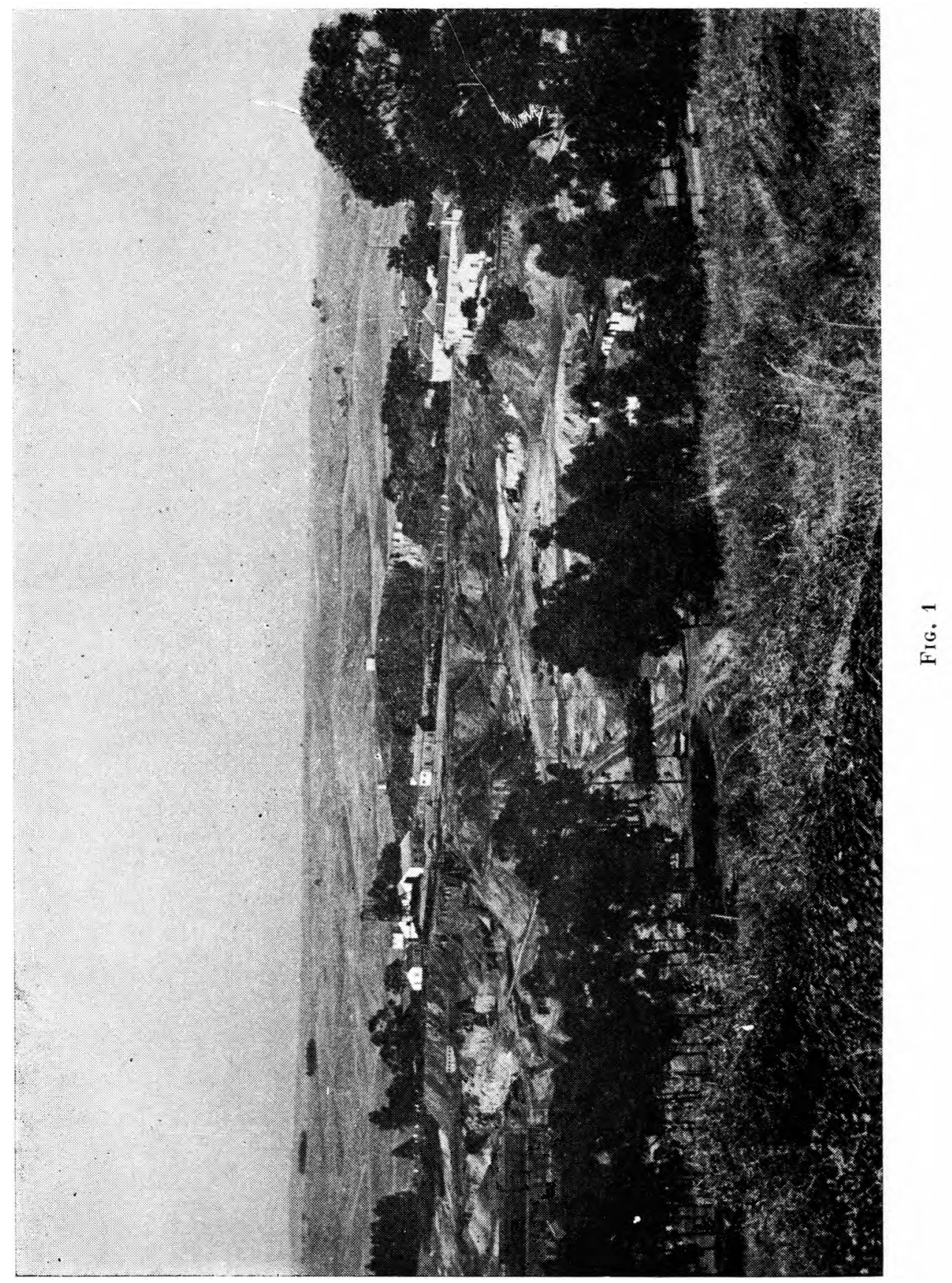




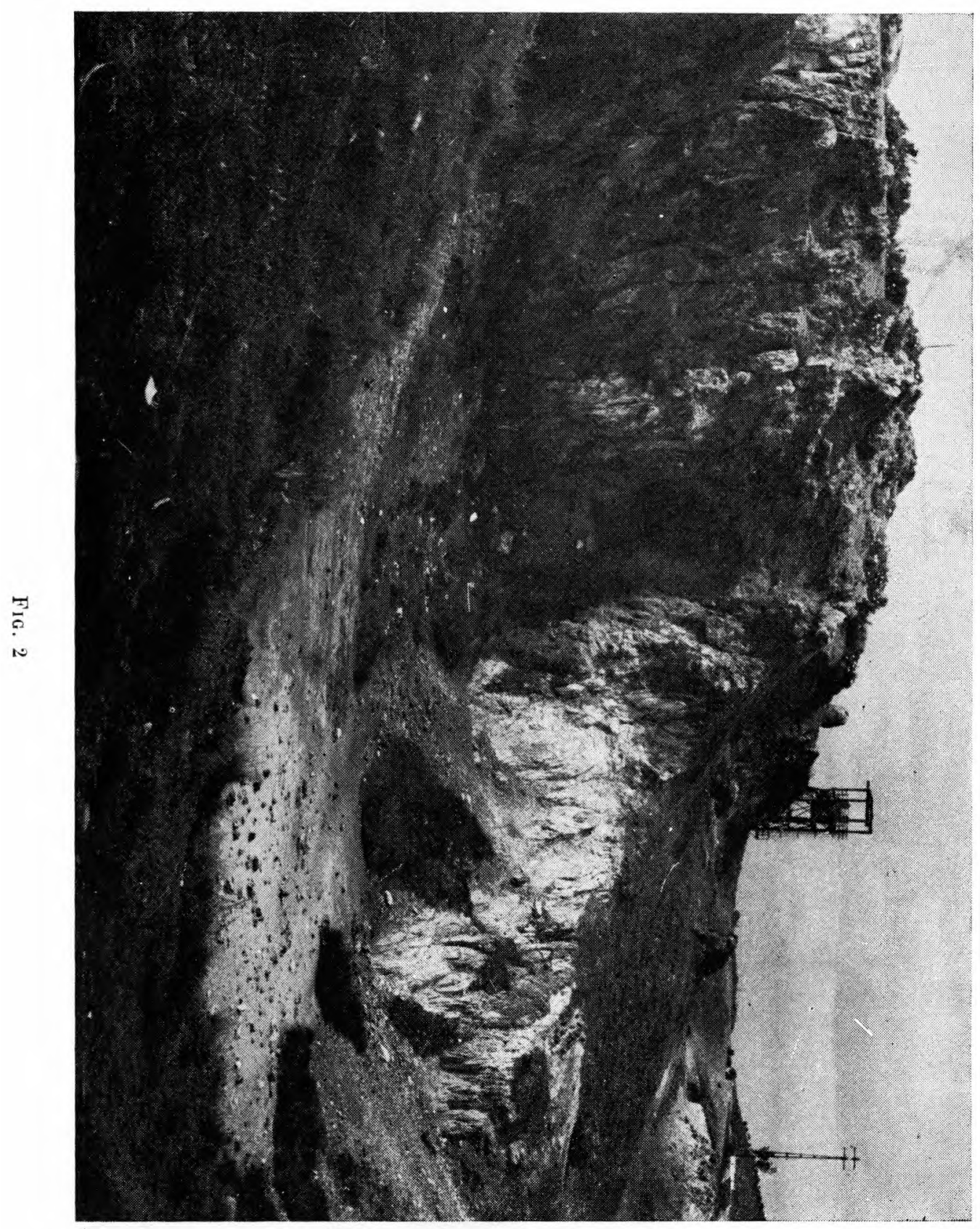




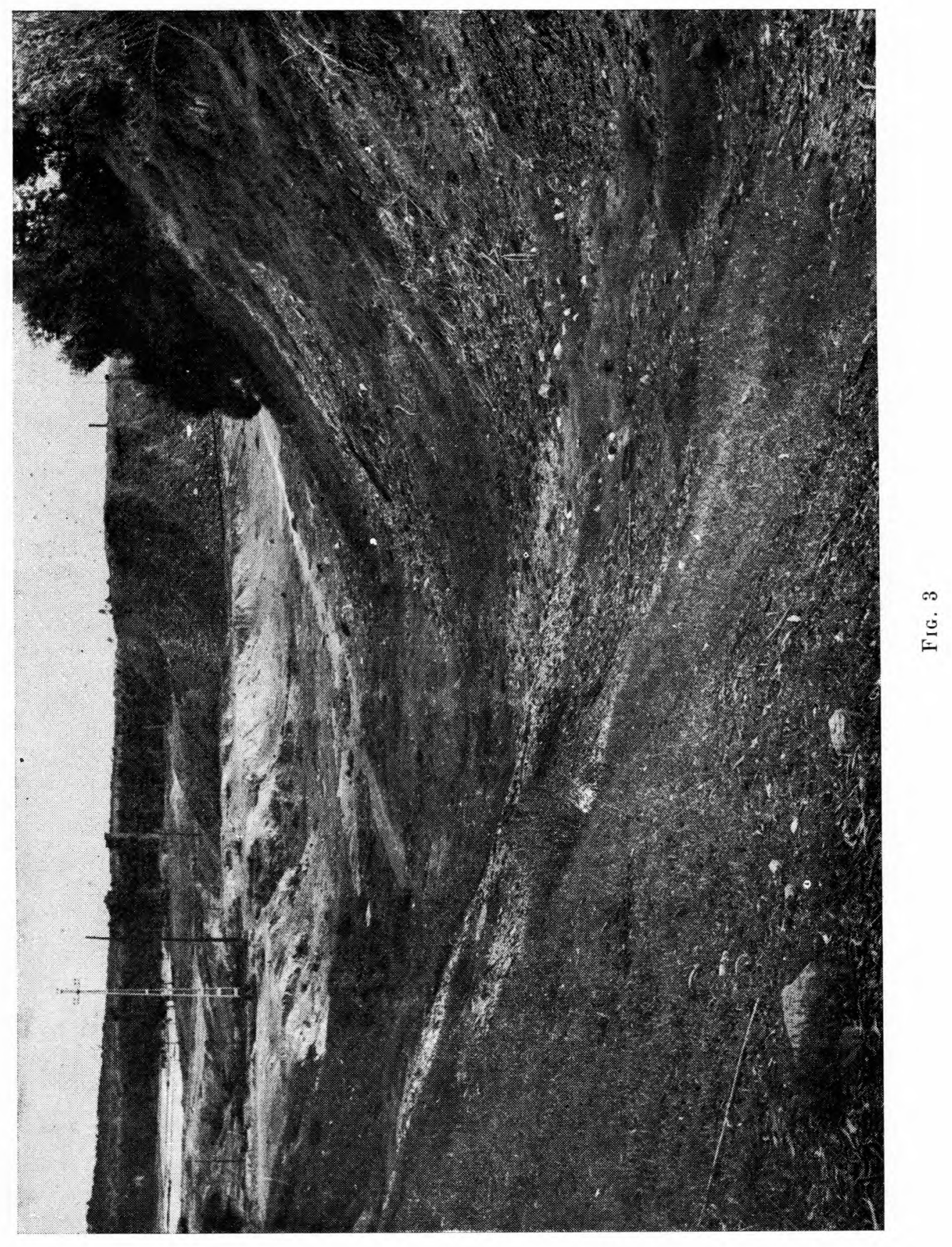




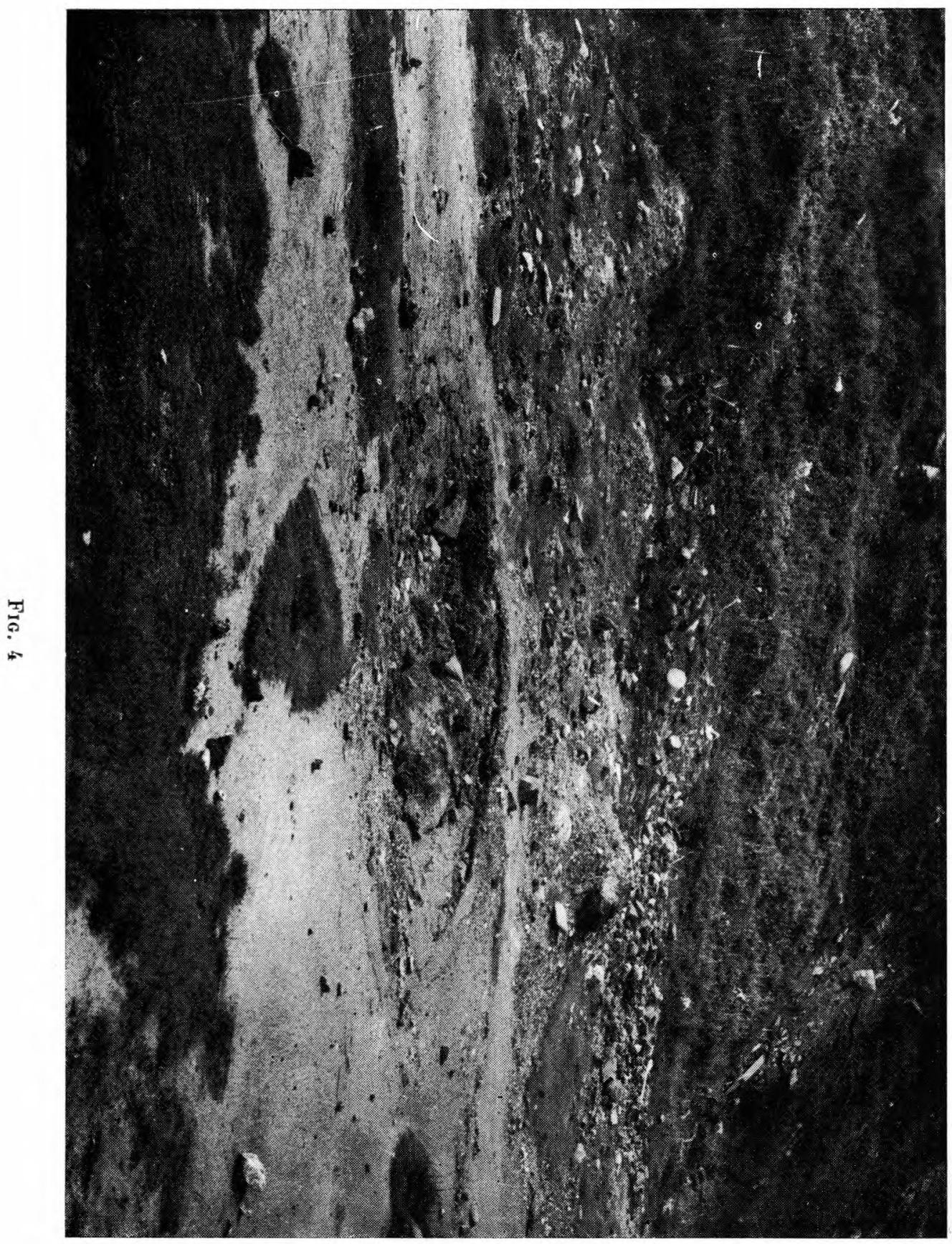




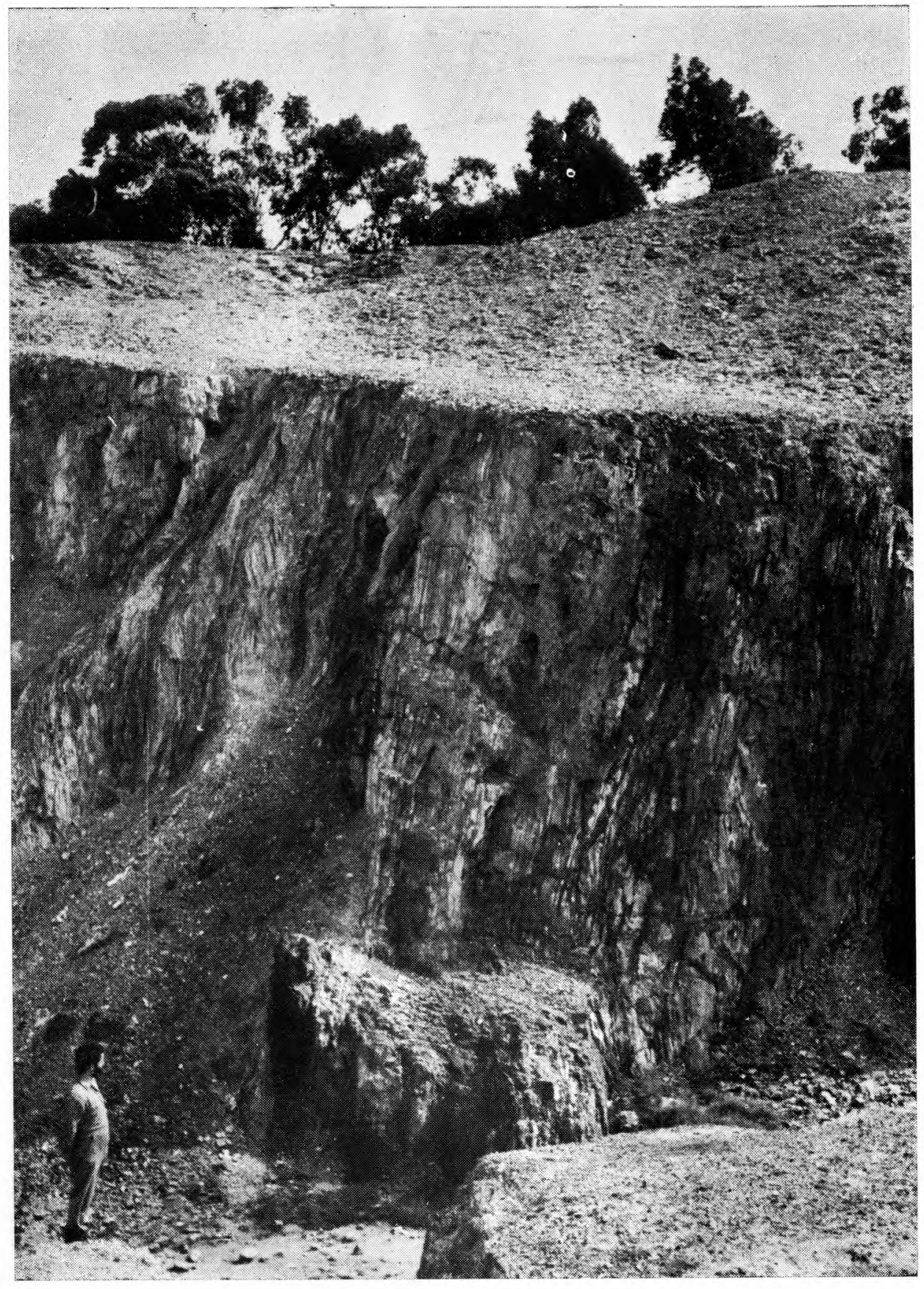

FIG. 5 


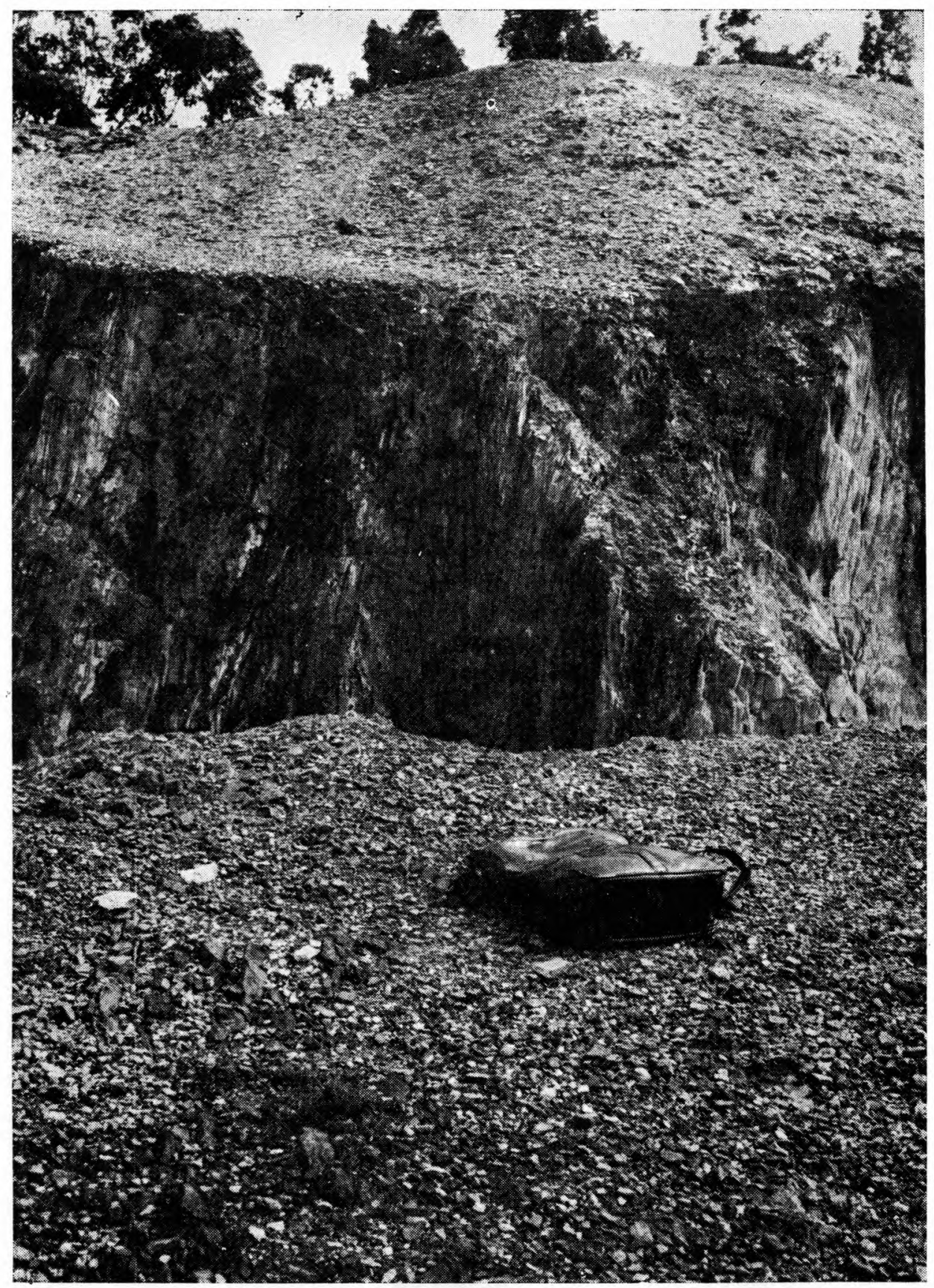

FIG. 6 


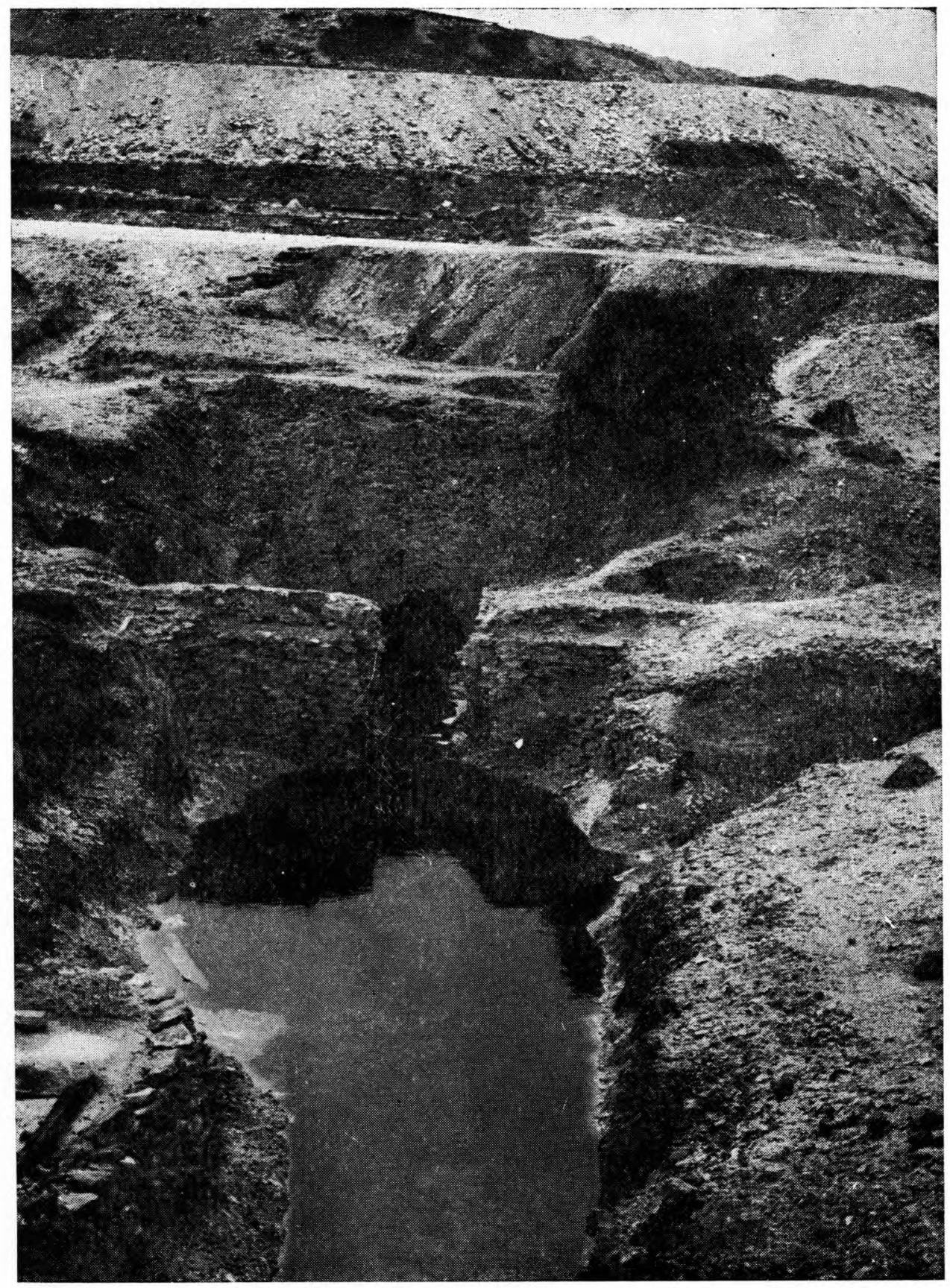

FIG. 7 


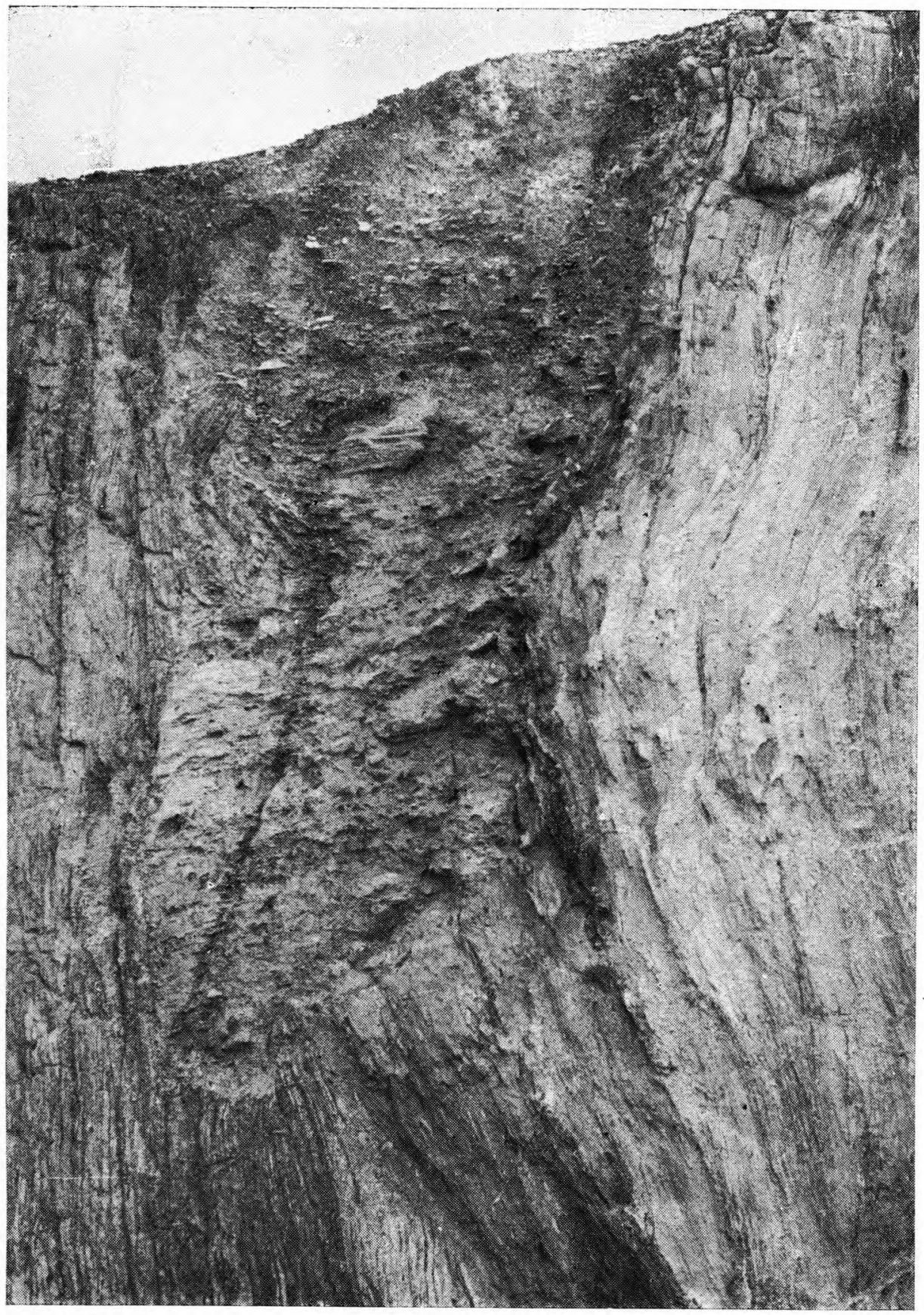

FIG. 8 


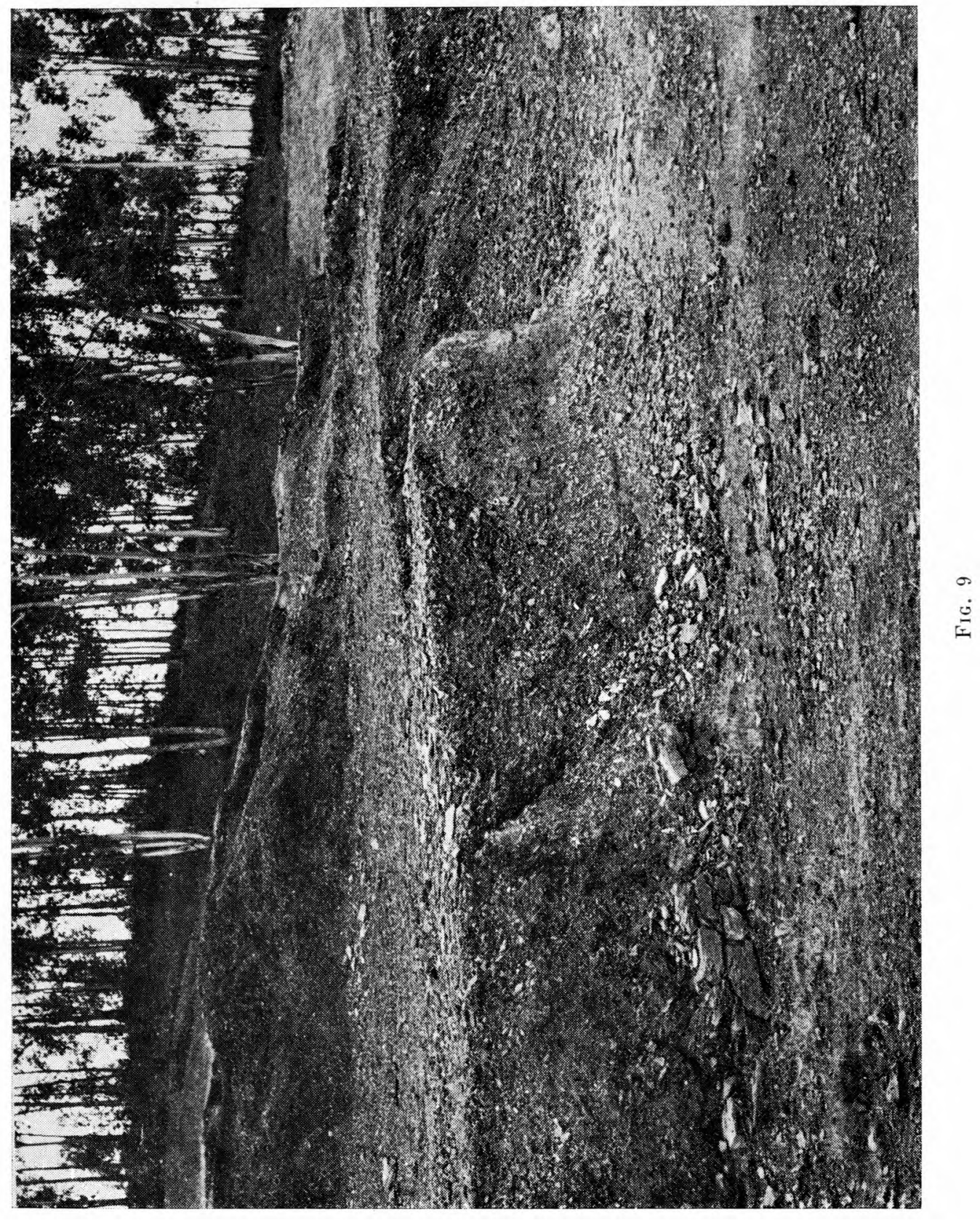




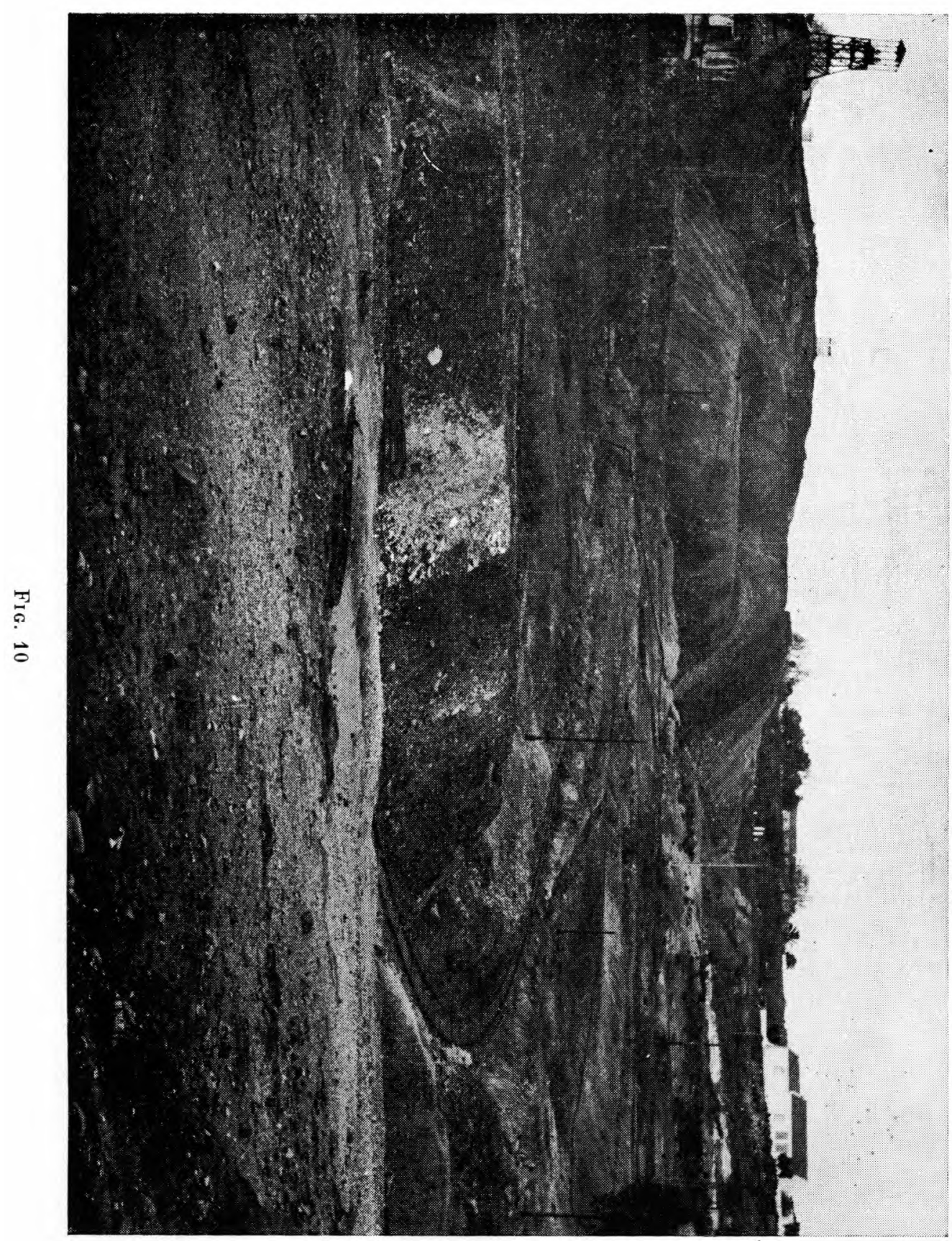




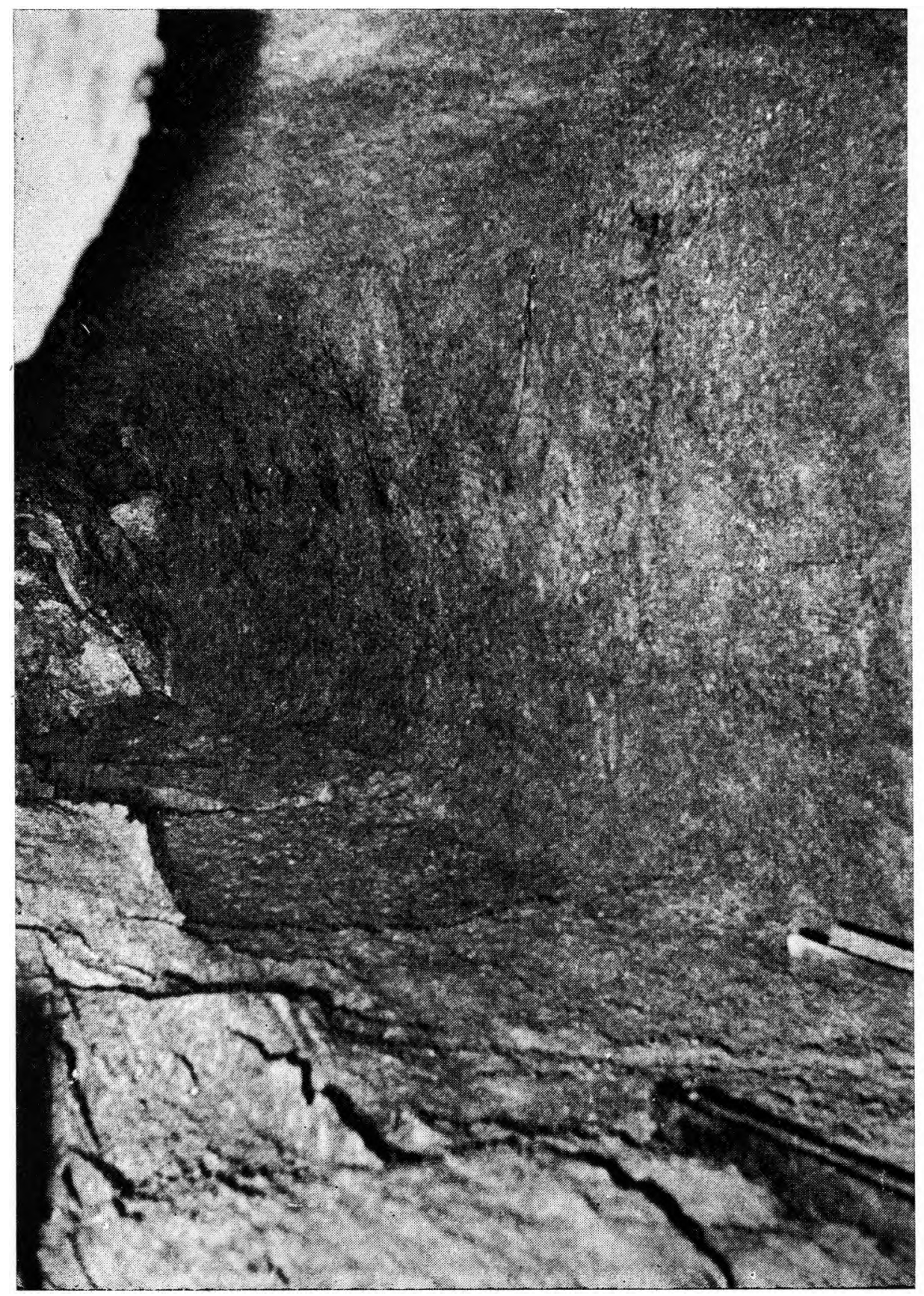




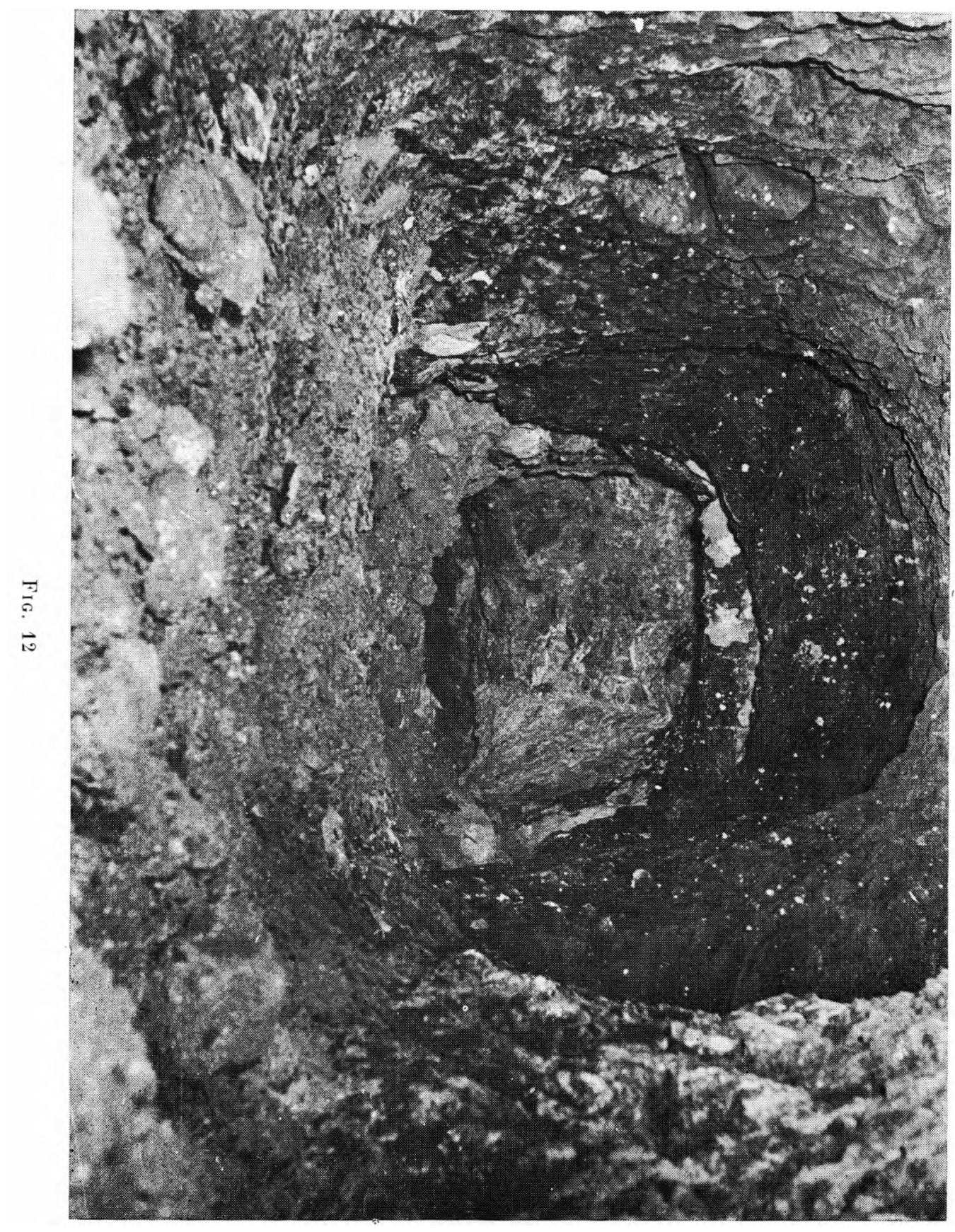




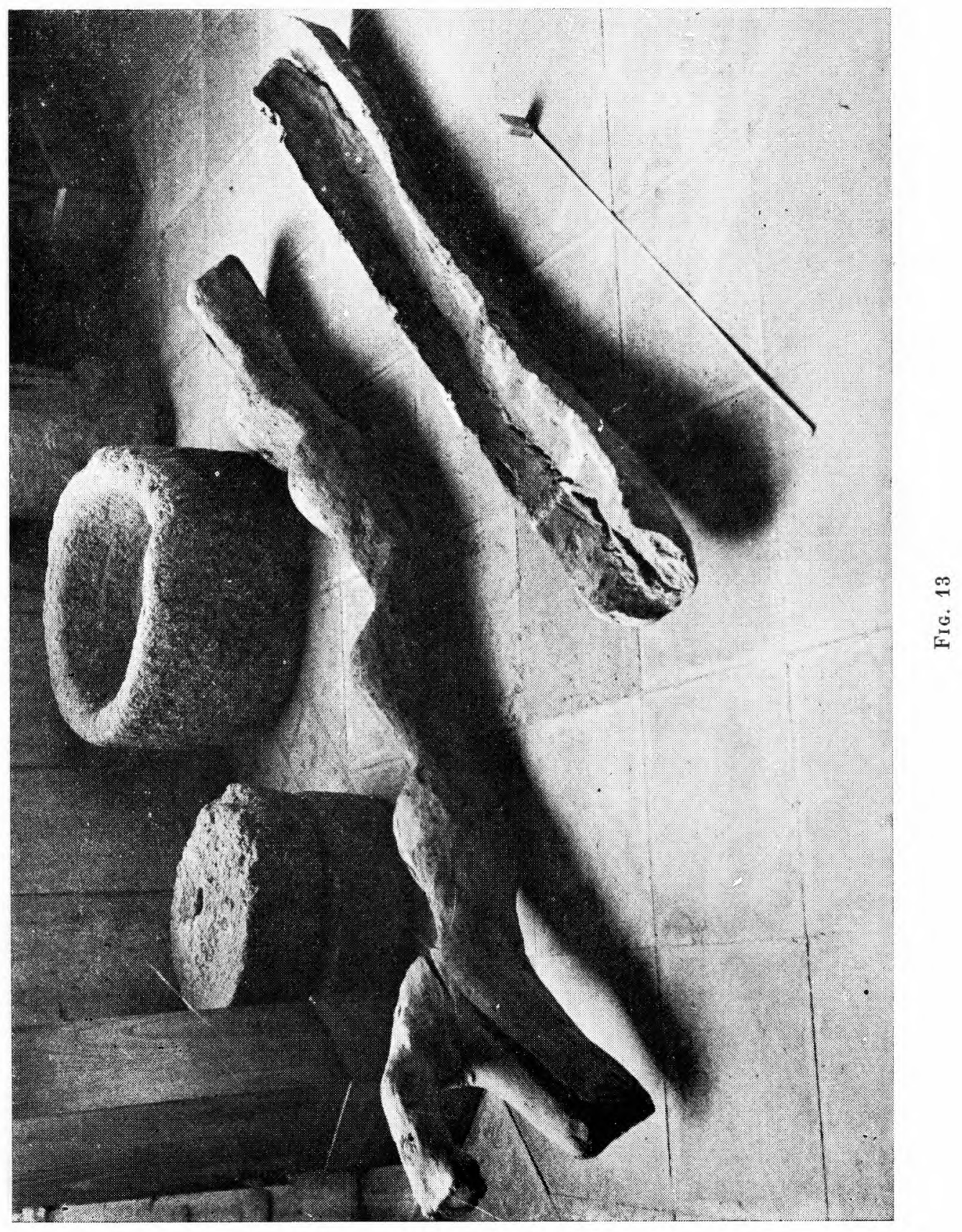




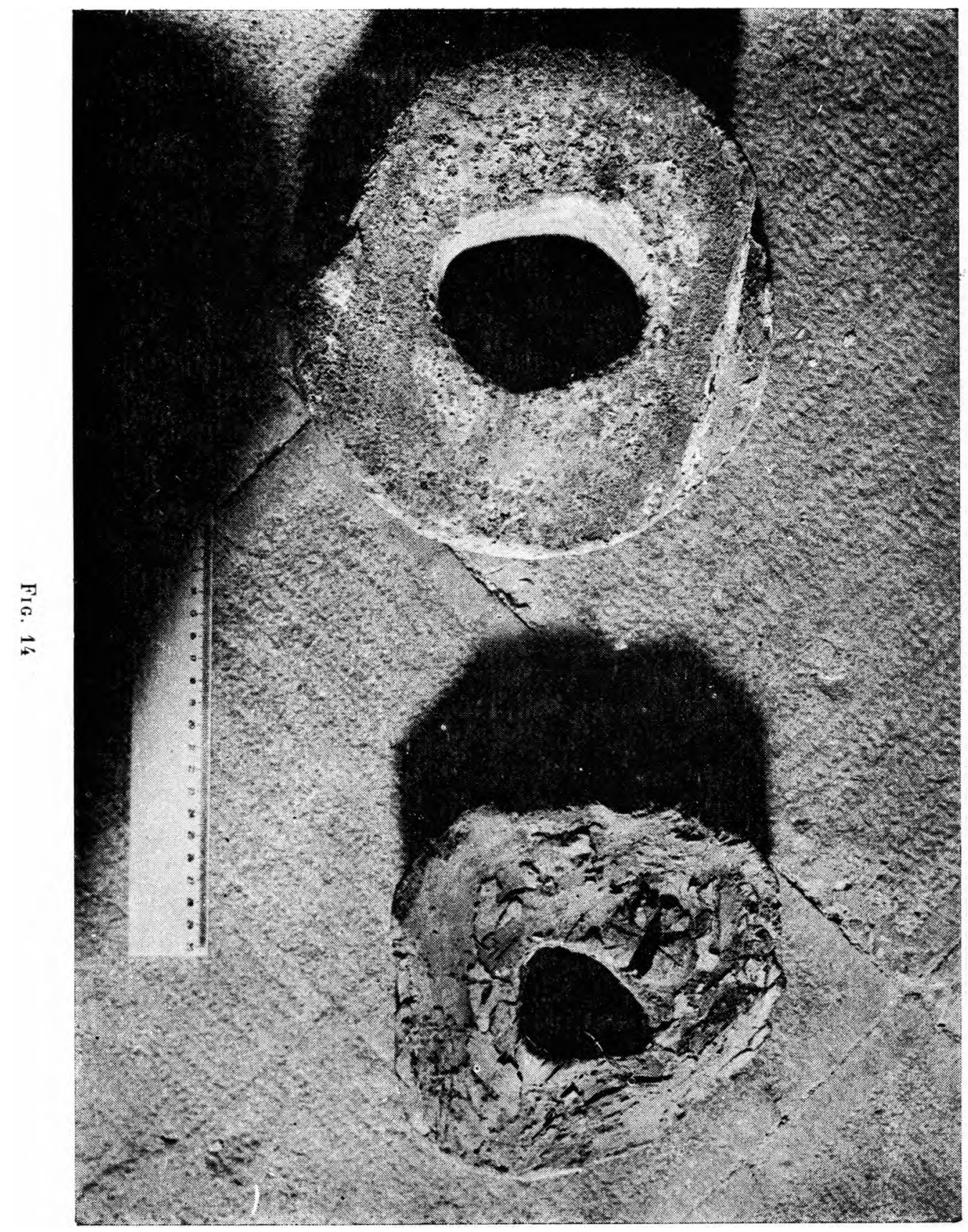




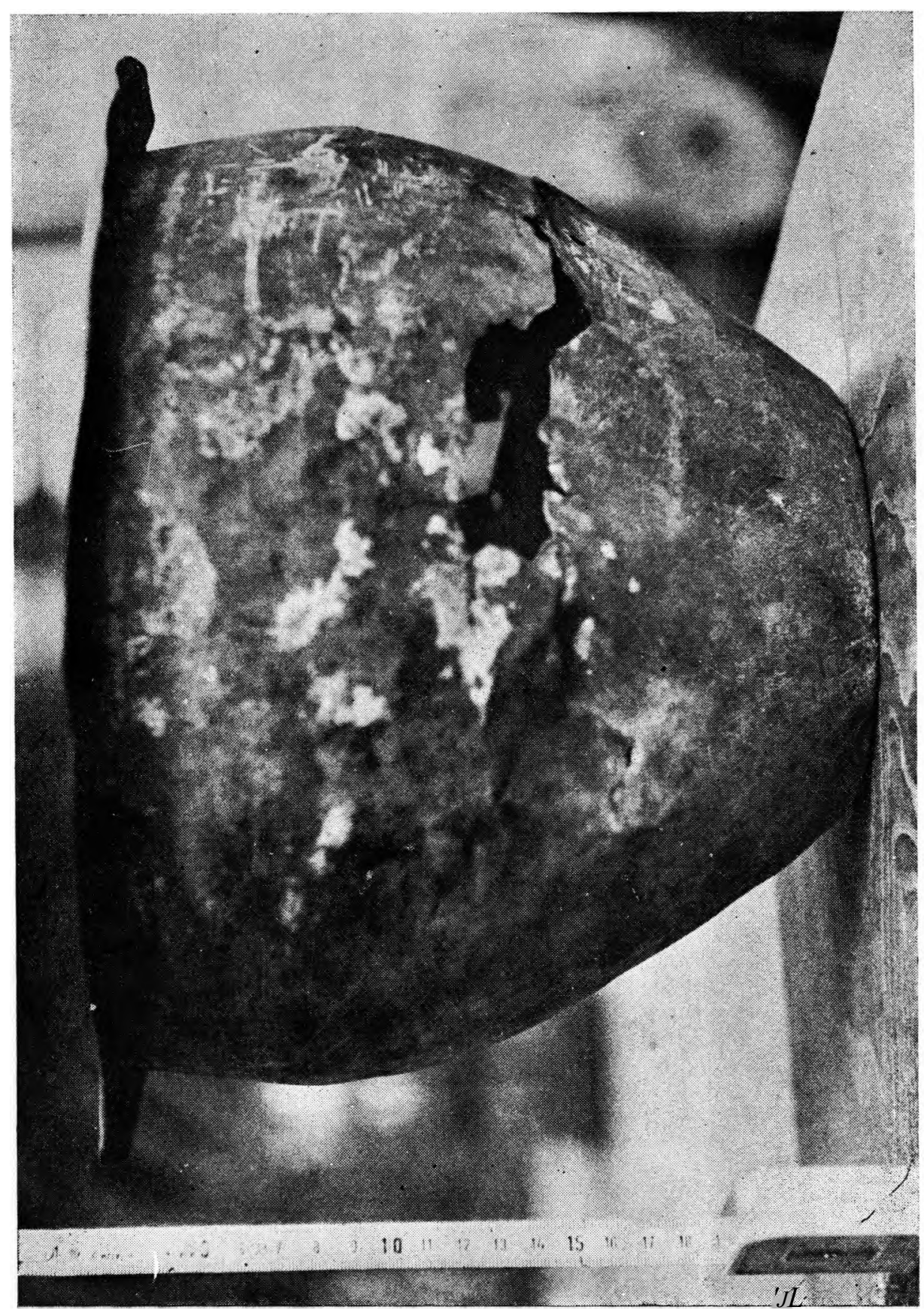

ำ 


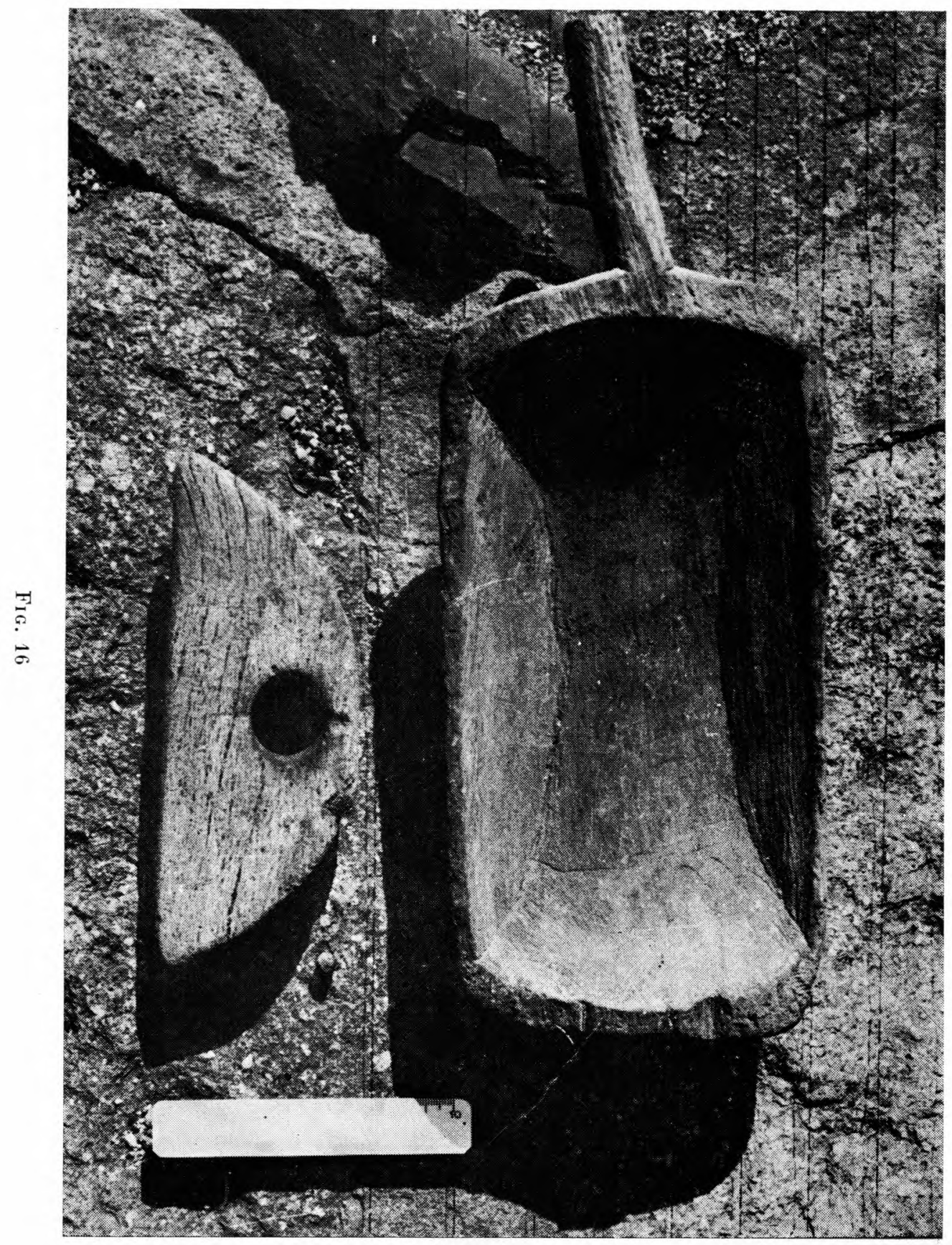




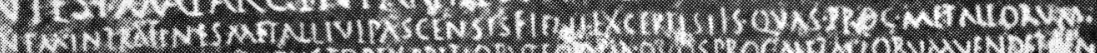

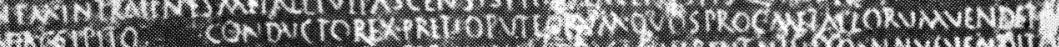

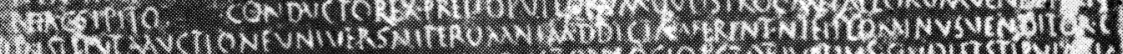

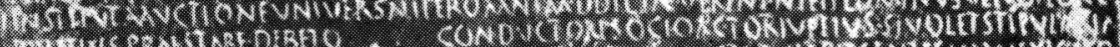

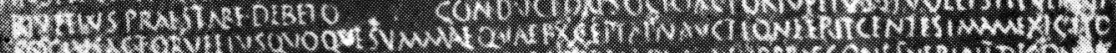

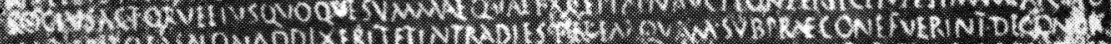

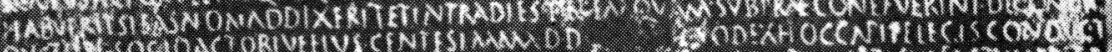

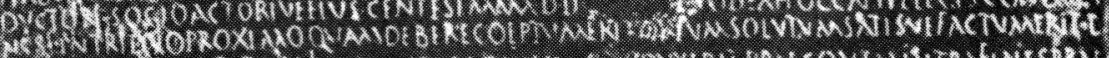

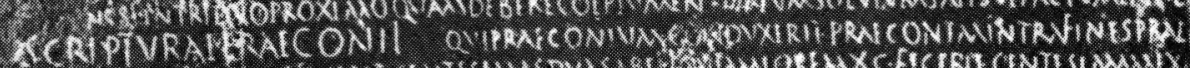

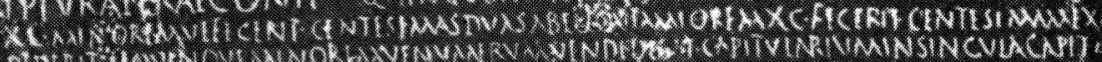
nivimet CWU

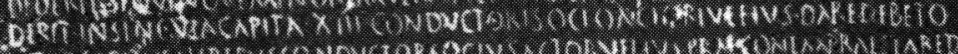
girogsin. (4)

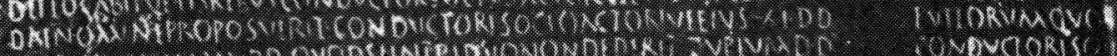

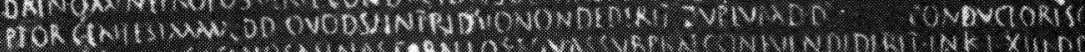

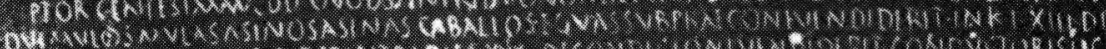

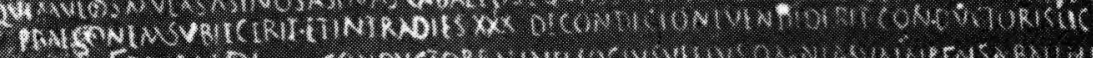

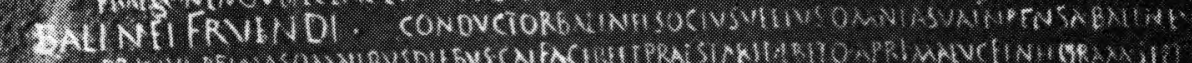

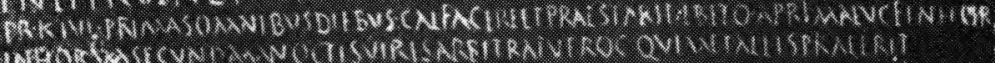

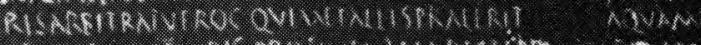

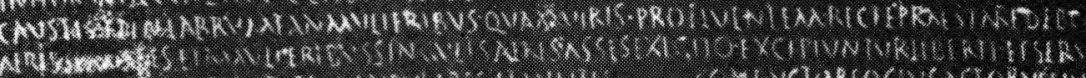

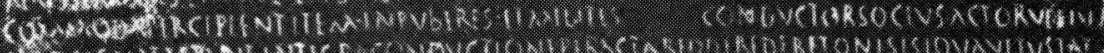

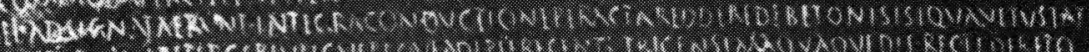

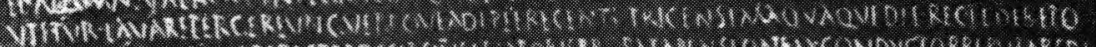

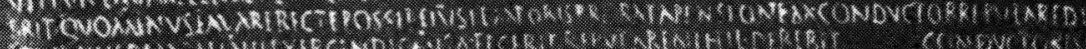

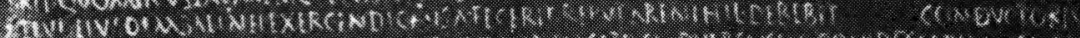

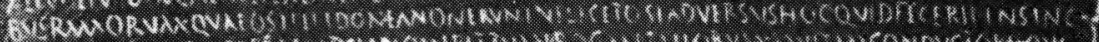

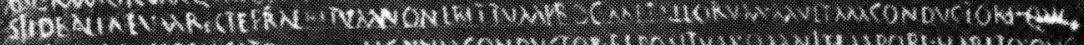

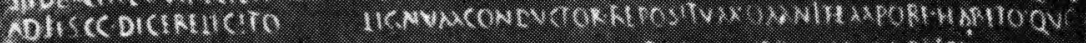

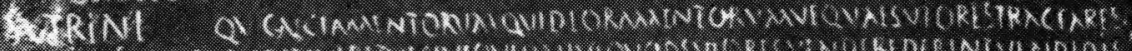

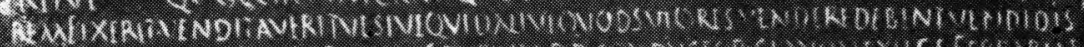

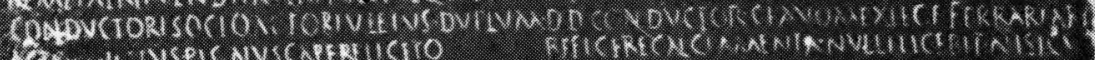

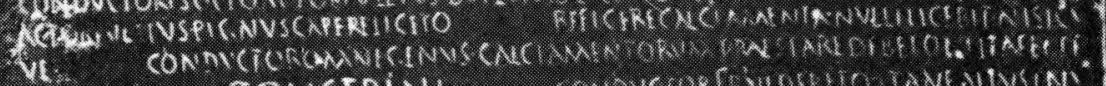
rrio TONSIRINI

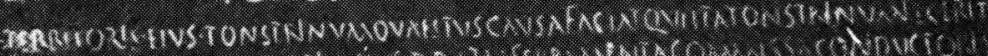

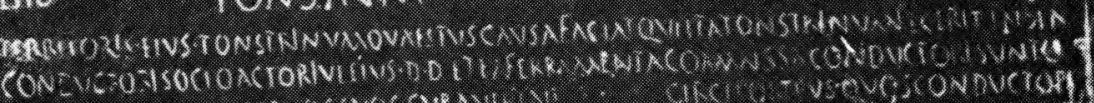

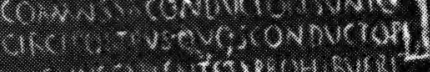

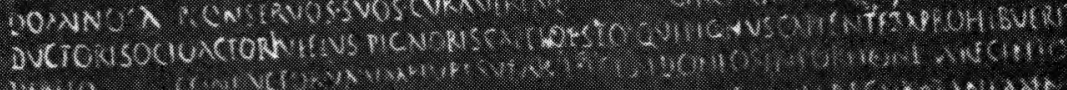

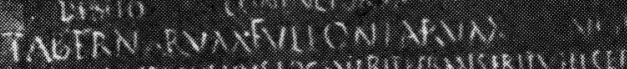

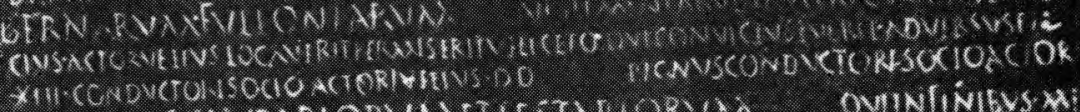

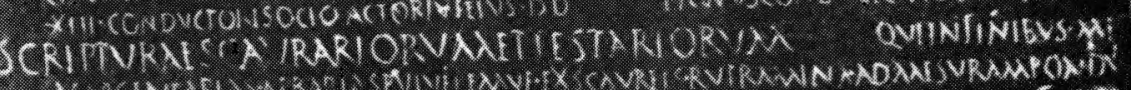

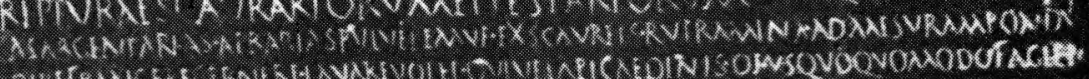

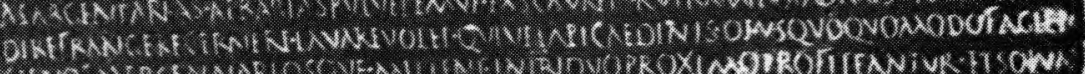

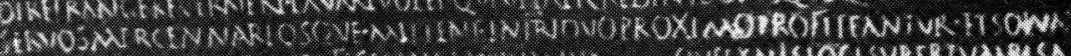

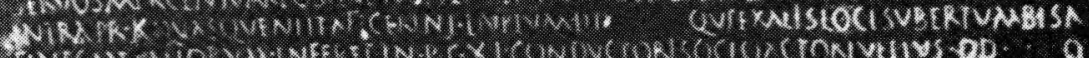

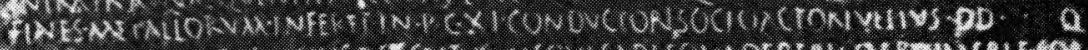

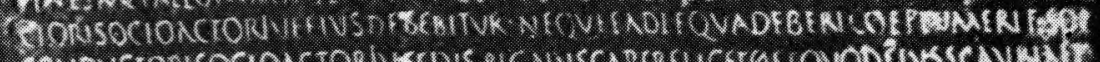

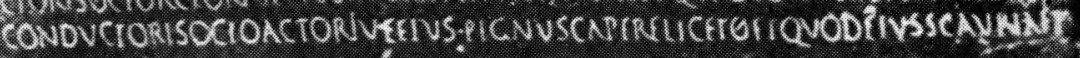




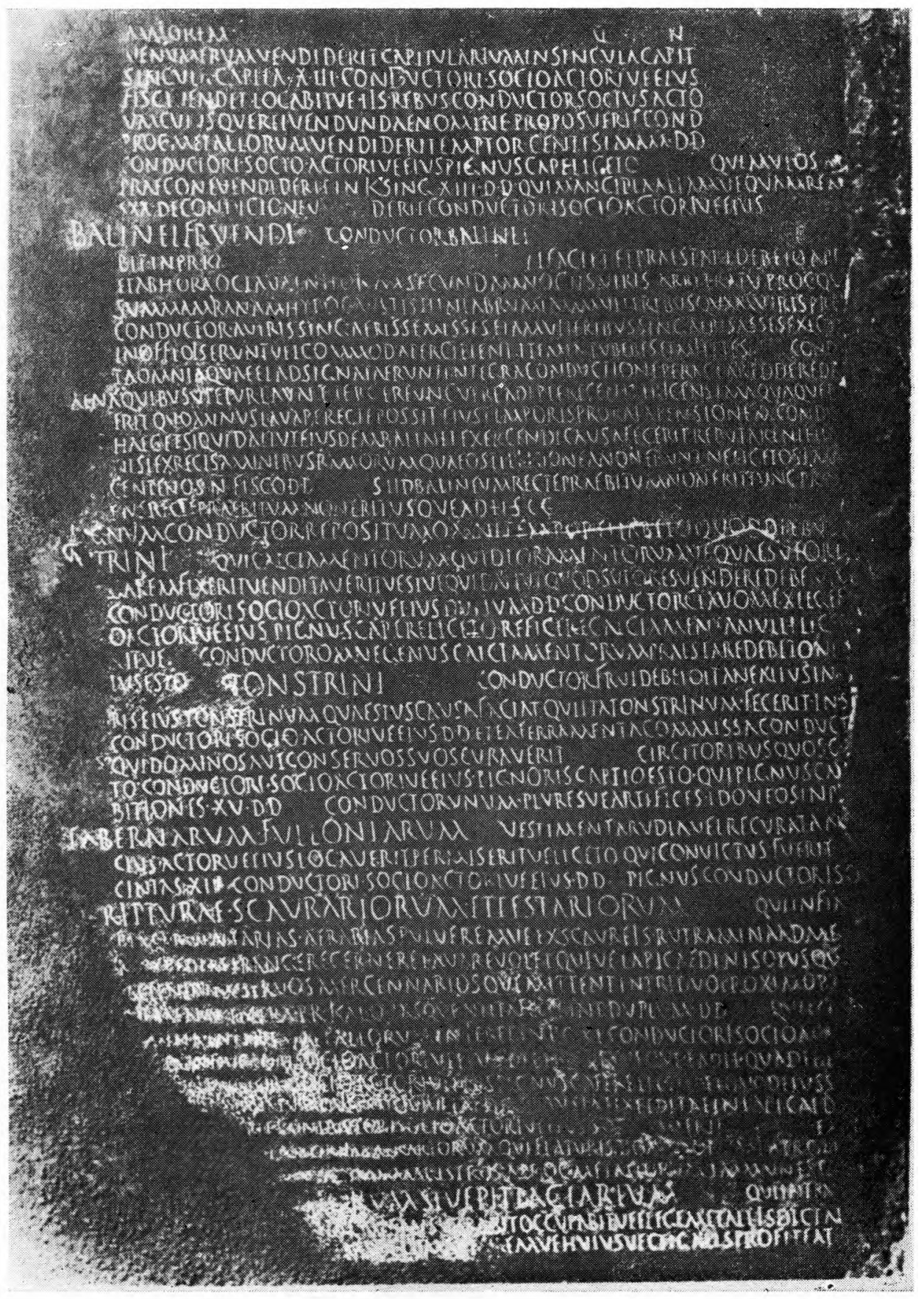

FIG. 18 


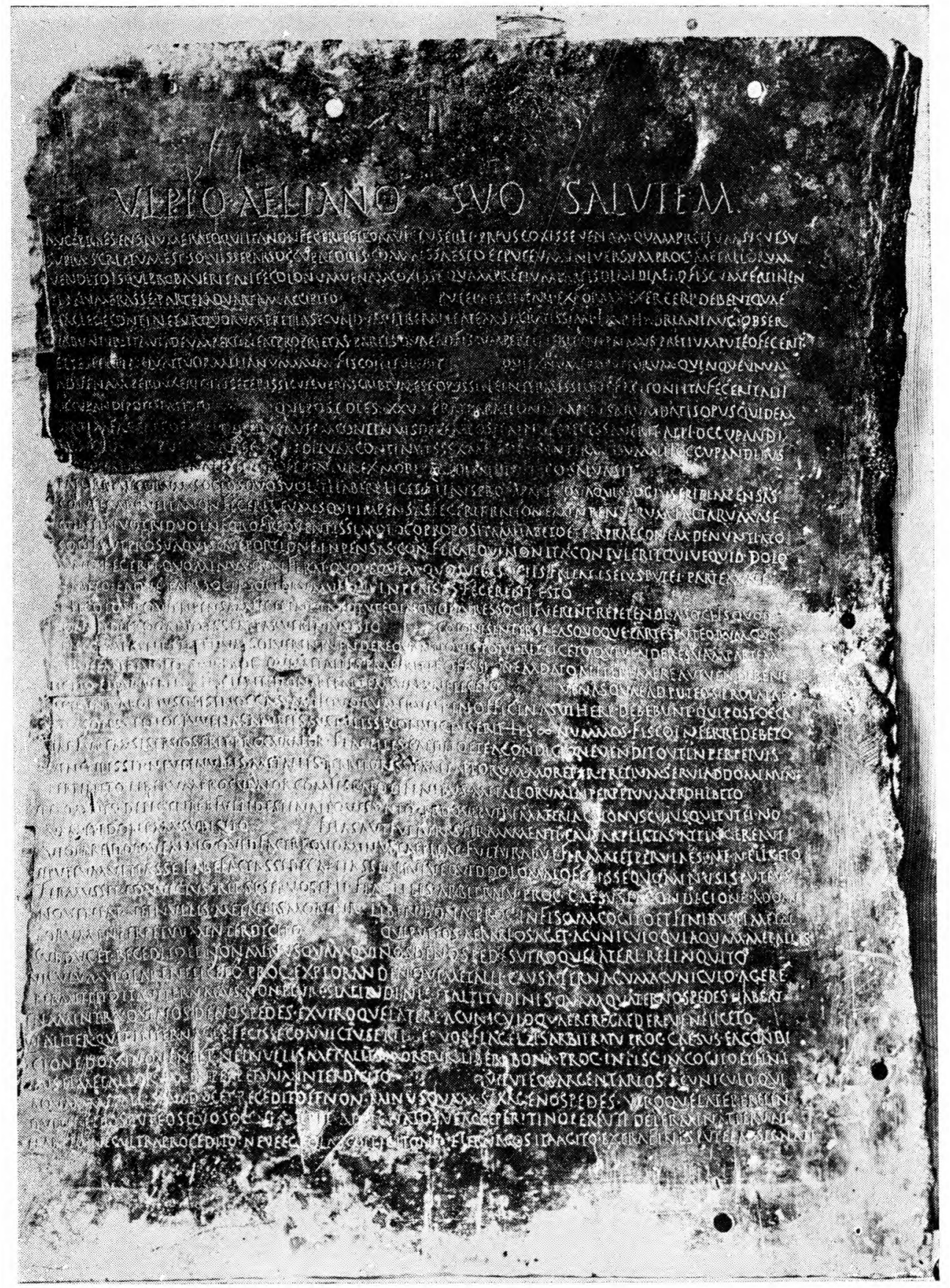




\section{OO}

CENTESIMAE ARGENTARIAE STIPVLATIONIS

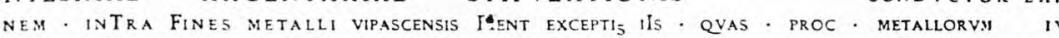

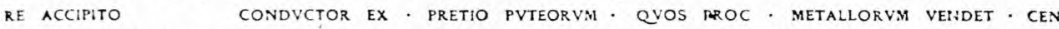

S! INSTITVTA AVCTIONE VNIVERSALITER ONINIA ADDICTA FVERINT * NIHILO MINVS VENDITOR * C

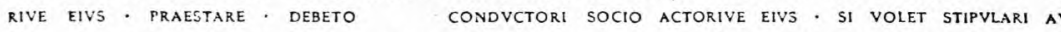

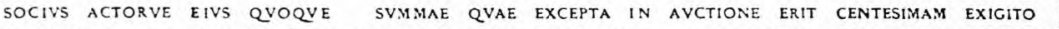

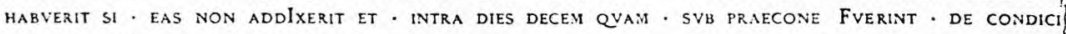
DVCTOR! SOCIO ACTORIVE EIVS - CENTESIMAM - D D D QVOD EX HOC CAPITE LEGIS CONDVCT NISI IN TRIDVO PROXIMO QVAM DEBERE COEPTVM ERIT DATVM SOLVTVM SATISVE FACTVM ERIT - D 10 SCRIPTVRAE PRAECONII QVI PRAECONIVM - CONDVXERIT P PRAECONEM INTRA FINES PRAEB X L - minoremve Fecerit - Centesimas dVas ab eo qvi maiorem x C F Fecerit - centesimam exic

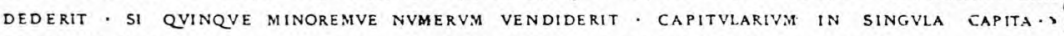

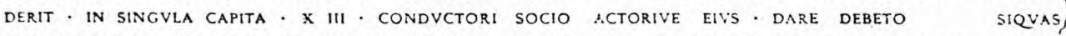
Det locabitVe - iIs rebvs condVCtor socivs actorve eIVs - praeconem fraestare debeto d DAE NOMINE PROPOSVERIT - CONDVCTORI SOCIO ACTORIVE EIVS $\cdot \mathrm{X} 1$. D D P PVTEORVM QVOD PTOR CENTESIMAM - D D - QVOD St IN TRIDVO NON DEDERIT - DVPLVM - D - D CONDVCTORI SOC

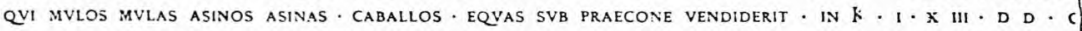
PRAECONEM SVBIECERIT - ET INTRA DIES - XXX - DE CONDICIONE VENDIDERIT - CONDVCTORI SOCIO

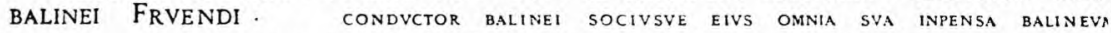

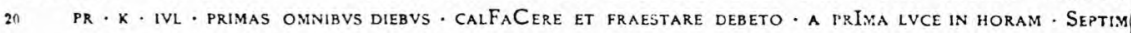
IN HORAM SECVNDAM NOCTIS VIRIS ARBITRATV PROC QVI METALLIS PRAEERIT A QVAM IN

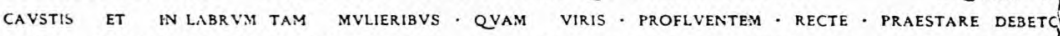

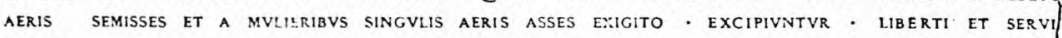
COMMODA PERCIPIENT ITEM - INPVBERES - ET MILITES CONDVCTOR SOCIVS - ACTORVE EIVS

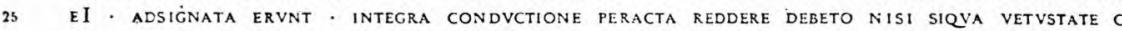
vtetvr - lavare tergere yngVereque adipe e recenti tricensima qVaqVe die - recte debeto ERIT QVO MINVS - LAVARE RECTE POSgIT EIVS TEMPORIS PRO RATA PENSIONEM - CONDVCTOR REPVTARE DEB ALIVT - EIVSDEM BALINEI EXERCENDI CAVSA - FECERIT - REPVTARE NIHIL - DEBEBIT CONDVCTORI VE' BVS RAMORVM - QVAE OSTILI - IDONEA NON ERVNT NE LICETO · SI ADVERSVS HOC QVID FECERIT * IN SING" SI ID BALINEVM - RECTE PRAEBITVM NON ERIT - TVM PROC - METAllorvM - MVLTAM CONDVCtORi - QVO AD HS CC - DiCERE LiCETO LignVM CONDYCTOR - REPOSITVM - OMNI teMPORE - habeto - QVO

SVTRINI QVi Calciamentorvm QVid loramentorvmVe eVae sVtores tractare sC

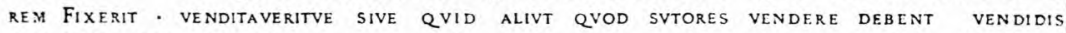
CONDVCTORI SOCIO ACTORIVE EIVS - DVPLVM D D CONDVCTOR - CLAVOM EX LEGE FERRAR!AR

35 ACTORIVE EIVS - PIGNVS CAPERE LICETO REFICERE CALCIAMENTA * NVLLI LiCEBIT * NISI CI VE CONDVCTOR OMNE GENVS - CALCIAMENTORVM PRAESTARE debeto Ni ITA Fecer ESTO TONSTRINI CONDVCTOR FRVI DEBETO - ITA NE ALIVS INI

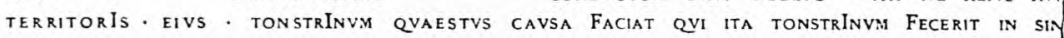
CONDVCTORI SOCIO ACTORIVE EIVS D D D . ET EA FERRAMENTA - COMMISSA CONDVCTORI SVNTO 46. DOMINOS AVT CONSERVOS - SVOS.CVRAVERINT CIRCITORIBVS - QYOS CONDVCTOR DVCTORI SOCIO ACTORIVE EIVS PIGNORIS CAPTIO ESTO Q QVI PIGNVS CAPIENTEM PROHIBVERIT DEBETO CONDVCTOR . VNVM PLVRESVE ARTIFICES IDONEOS IN PORT:ONEM RECIPITO

TABERNARVM. FVLLONIARVM VESTIMENTA RVDIA VEL RECVRATA Nemini civs - actorve eivs locaverit permiseritve liceto - QVi convictVs fverit - adversvi ea QVi $X$ III CONDVCTORI SOCIO ACTORIVE EIVS - D D PIGNVS CONDVCTORI - SOCIO ACTORI SCRIPTVRAE SCAVRARIORVM ET TESTARIORVM QVI IN FINIBVS - MET as argentarias - aerarias pVlveremve ex scavreis - rvtramina - an mesvram ponidv dire frangere cernere l lavare volet - QVive lapicaedinis opvs a qu QVo modo facien SERvos mercenNariosqVe. MitTent - in tridVo proximo profiteantVR - et solvan

so INTRA PR. K QVASQVE NI ITA FECERINT D DVPLVIA D D QVI EX A ALIS LOCIS VBERTVMBiSAE

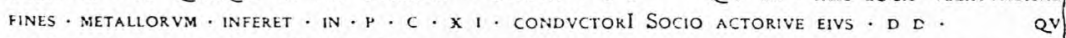
CTORI SOCIO ACTORIVE EIVS DEBEBITVR . NEQVE EA DIE QVA DEBERI COEPTVM ERIT - SOLY CONDVCTORI SOCIO ACTORIVE EIVS. PIGNVS CAPERE LICETO FT QVOD EIVS SCAVRIAE PV 
MAIOREM -

VE NVMERVM VENDIDERIT CAPITVLARIVM IN SINGWLA CAPIT SINGVLA - CAPITA $\cdot \mathrm{x} \cdot 111 \cdot$, CONDVCTORI - SOCIO ACTORIVE EIVS FISCI VENDET - LOCABITVE - IIS - REBVS CONDVCTOR SOCIVS ACTO 5 VM CVIVSQVE REI VENDVNDAE NOMINE - FHOPOSVERIT COND PROC - METALIORVM VENDIDERIT EMPTOR - CENTESIMAM - D - D CONDVCTORI - SOCIO - ACTORIVE EIVS PIGNYS CAPE LICETO PRAECONE VENDIDERIT IN $\mathrm{K}$. SING $\mathrm{X}$ iH. D - D - QVI MANCIPIA - ALIAMVE QVAM REN XXX. DE CONDICIONE V DERIT . CONDVCTORI SOCIO ACTORIVE EIVS

10 BALINEI FRVENDI CONDVCTOR BNLINEI BIT - IN PR . KA ILFACIET ET PRAESTARE DEBETO A PRI ET AB HORA OCTAVA IN HORAM SECVNDAM NOCTIS VIRIS AREITRATV P PROC Q QV SVMMAM RANAM HYPOCAVSTIS ET IN LABRVM TAM MVLIERIBVS QVAM VIRIS PRO) CONDVCTOR - A VIRIS SING AERIS - SEMISSES ET A MVLIERIBVS - SING AERIS ASSES EXIGIT?

15 IN OFFIOIS ERVNT VEL COMMODA PERCIPIENT - ITEM INPVBERES ET MILITES. CONDI. TA OMNIA QVAE EI ADSIGNATA ERVNT INTEGRA CONDYCTIONE PERACTA REDDERE DE AENA QVIBVS VTETVR LAVARE TERGERE VNGVERE ADIPE E RECENTI TRICENSIMA QVAQVE D ERIT QVO MINVS LAVARE RECTE POSSIT - EIVS TEMPORIS FRO RATA PENSIONEM - CONDVC

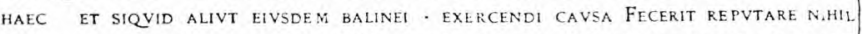

20 NISI EX RECISAMINIBVS RAMORVM QVAE OSTILI IDONEA NON ERVNT NE LICETO SI ADV CENTENOS TO FISCO-D.D. SI ID BALINEVM RECTE PRAEBITVM NON ERIT TVNC PFOCY ENS RECTE PRAEBITYM NON ERIT VSQYE AD HS CC DICERE LICETO

LIGNVM CONDVCTOR REPOSITVM OMNI TEMPORE HABETO QVOD DIEBVS

SVTRINI QVI CALCIAMENTORVM C GVID LORANENTORVMVE QVAE SVTORE

25 Garem Fixerit VenditaVeritVE SIVE QVID ALIVT QVOD SVTORES VeNDERE DEBENT VE CONDVCTORI SOCIO ACTORIVE EIVS DUPLVM - D - D CONDVCTOR CLAVOM EX LEGEH

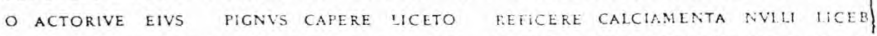
RITVE CONDVCTOR OMNE GENVS CALCIAMENTORVM FRAESTARE DEBETO N:I IVSESTO TONSTRINI CONDVCTOR FRVI DEEETO ITA NE ALIVS TNY!

30 RIS EIVS TONSTRINVM QVAESTVS CAVSA FACIAT QVI ITA TONSTRINIM - FECLRIT - IN SI CONDVCTORI SOCIO - ACTORIVE EIVS - D - D. ET EA FERRAMERTA COMAIISSA CONDVCT\} QVI DOMINOS AVT CONSERVOS SVOS CVRAVERIT CIRCITORIEVS CVOS C) TO CONDVCTORI. SOCIO ACTORIVE EIVS. PIGNORIS CAPTIO ESTO . QVI PIGNVS CAI BITIONES - $x V \cdot D D$ CONDVCTOR VNVM PIVRESVE ARTIFICES * IDONEOS IN I 35 TABERNARVM. FVLLONIARVM VESTIMENTA RVDIA VEl RECVRATA N CIVS ACTORVE EIVS LOCAVERIT PERMISERITVE LICETO QVI CONVICTVS - FVERIT * CINIAS A II CONDVCTORI - SOCIO ACTORIVE EIVS. D D . PIGNVS CONDVCTORI SOC

SCRIPTVRAE - SCAVRARIORVM - ET TESTARIORVM QVI IN EINIM RIAS ARGENTARIAS - AERARIAS PVLVEREMVE EX SCAVREJS - RVTRinitiNa AD MES/

40 RE EXPEDIRE FRANGERE CERNERE laVARE VOLET Q QVIVE - LAPICAEDINIS OPVS QVy FACIENDVM SERVOS MERCENNARIOSQVE MITTENT IN TRIDVO PROXIMO PR QVE MENSE INTRA - PR - KAL - QVASQVE - NI - ITA FECERINT DVPLYM - D D QVIEX AL TRAMINA IN FINES METALLORVM INEERET IN $\mathrm{P} \quad \mathrm{C} \mathrm{X} I$. CONDVCTORI . SOCIO ACTO LEGIS CONDVCTORI SOCIO ACTORIVE EIVS DEBEEITVR. NEQVE . EA DIE - QVA DEBEF

45 D D D CONDVCTORI SOCIO ACTORIVE EIVS PIGNVS CAPERE LICETO. ET QVOD EIVS SC TVM CRETVM LAVATVMQVE ERIT QVIVE LAPIDES LAVSTAE EXPEDITAE IN IAPICAEDI QVID DEBITVM ERIT CONDVCTORI SOCIO ACTORIVE EIVS - SOLVTVM ERIT FLATORVM ARGENTARIORVM AERARIORVM QVIELATVRIS DOMINORVM PATRON LVDI MAGISTRI LVDI MAGISTROS . A PROC . METALIORYM IMMVNES ESS 50 VSVRPATIONES PVTEORVM SIVE. PITTACIARIVM QVI INTRA F QVE PVTEI iVRIS RETINENDI CAVSA VSVRPABIT OCCVPABITVE E LEGE METALLIS DICTA E VERIT APVD CONDVCTOREM SOCIVM ACTOREMVE HVIVS VECTIGALIS IROFITEATV 


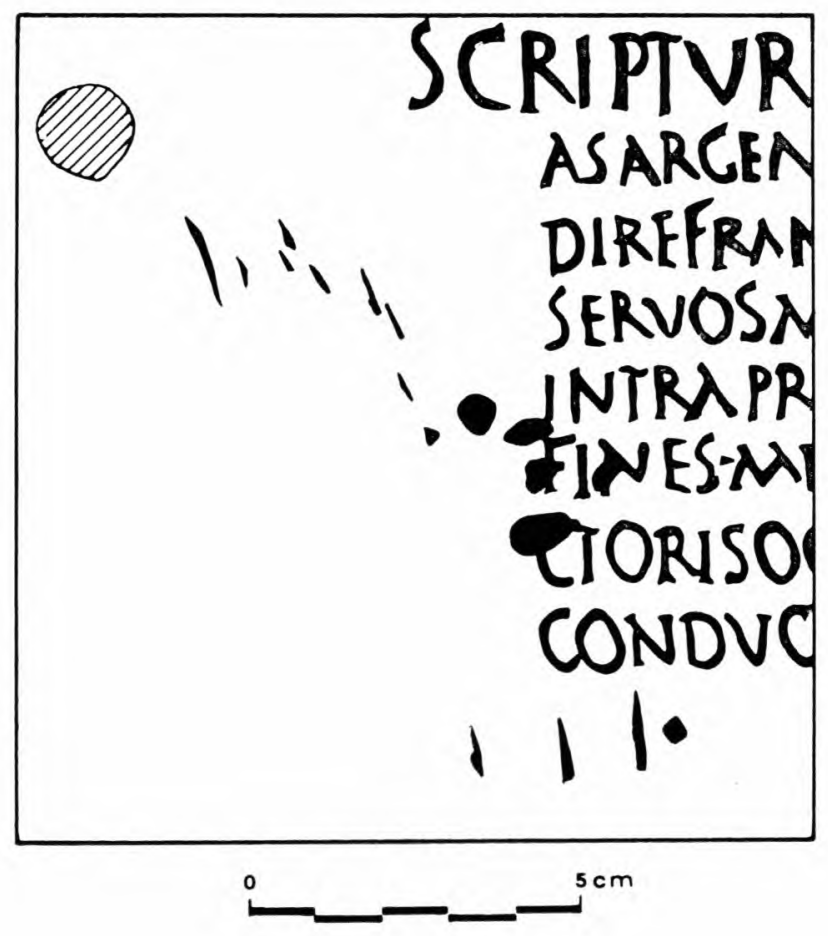

FIG. 22 

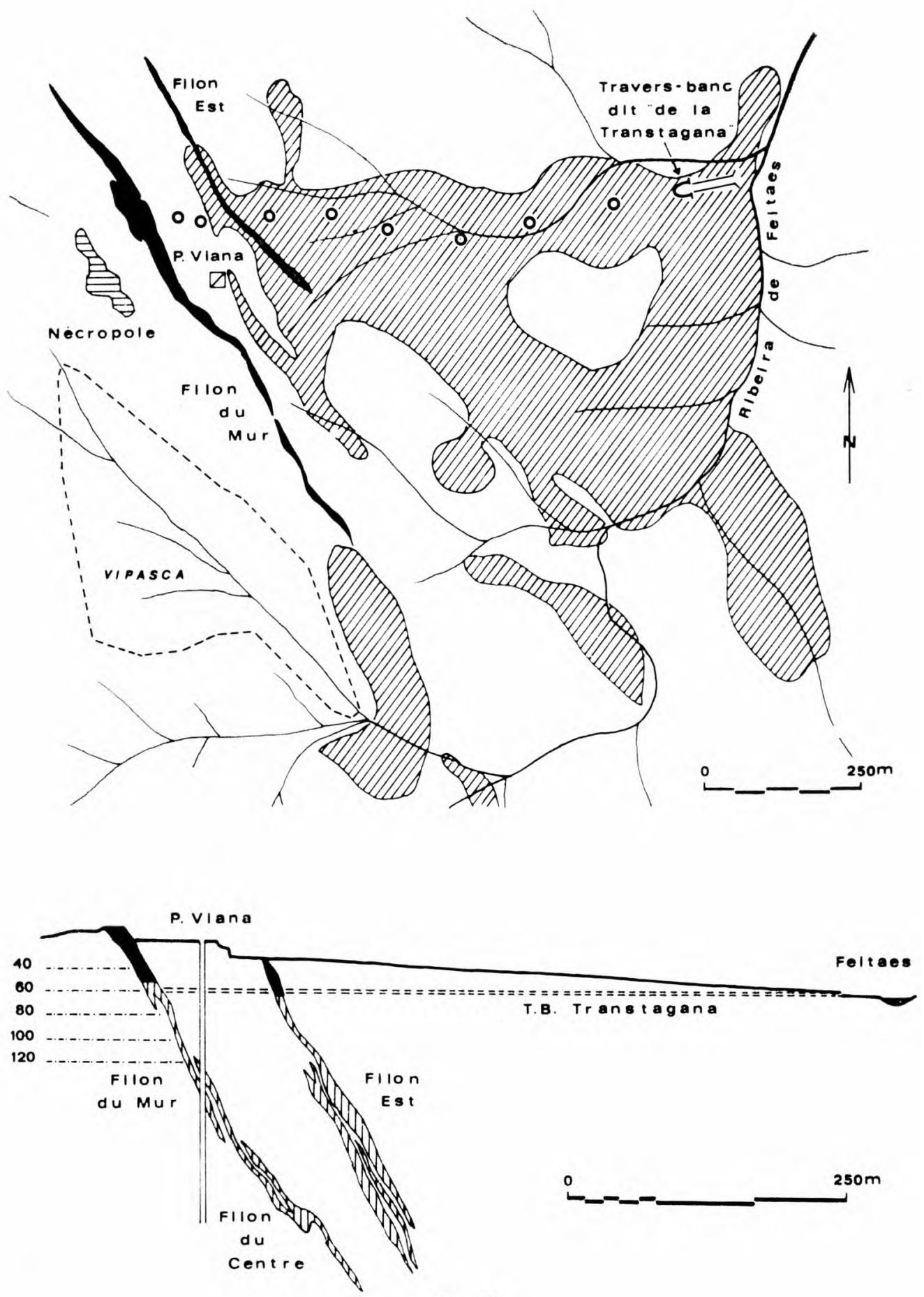

FIG. 23 
(Página deixada propositadamente em branco) 


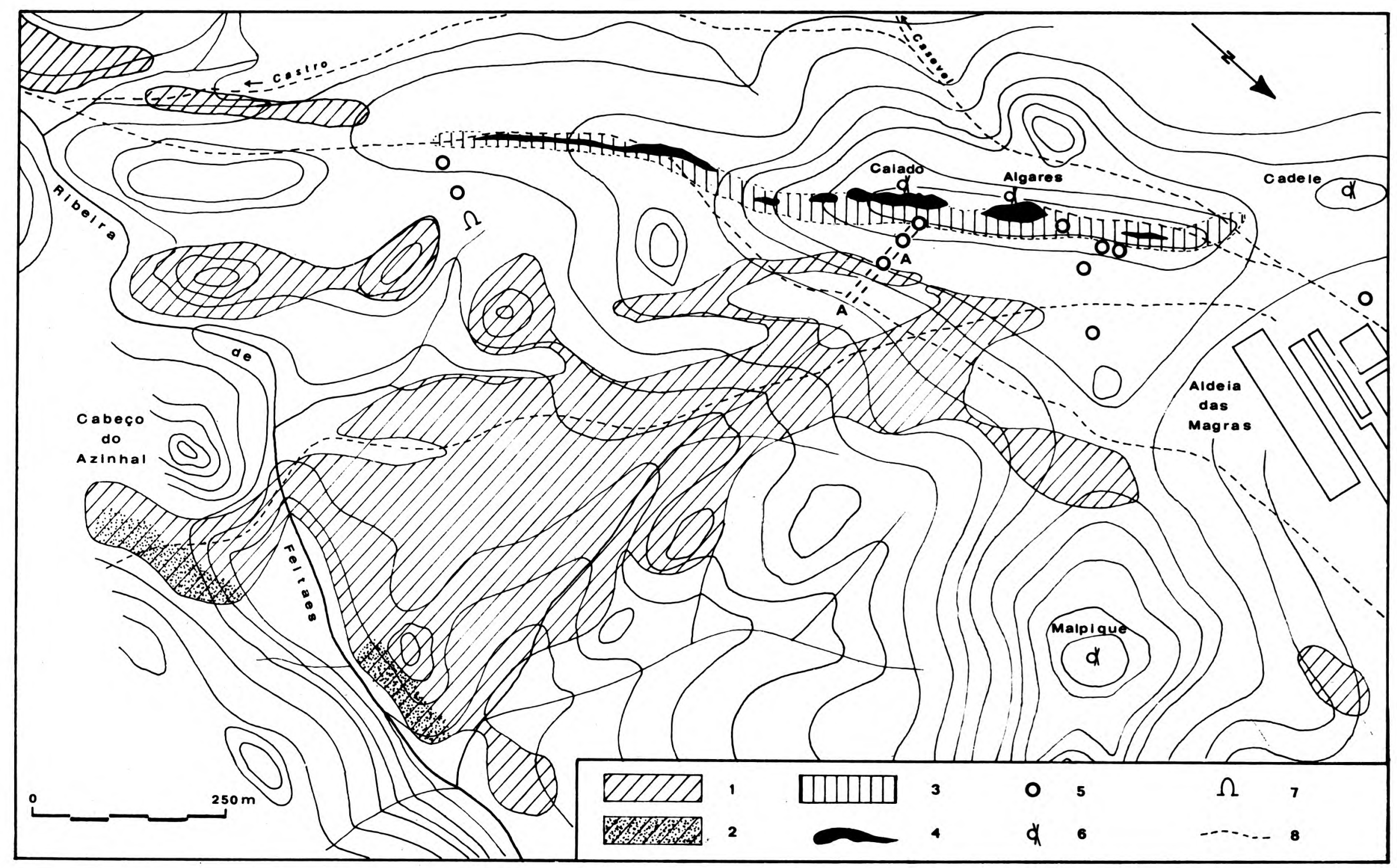

FIG. 24 
(Página deixada propositadamente em branco) 


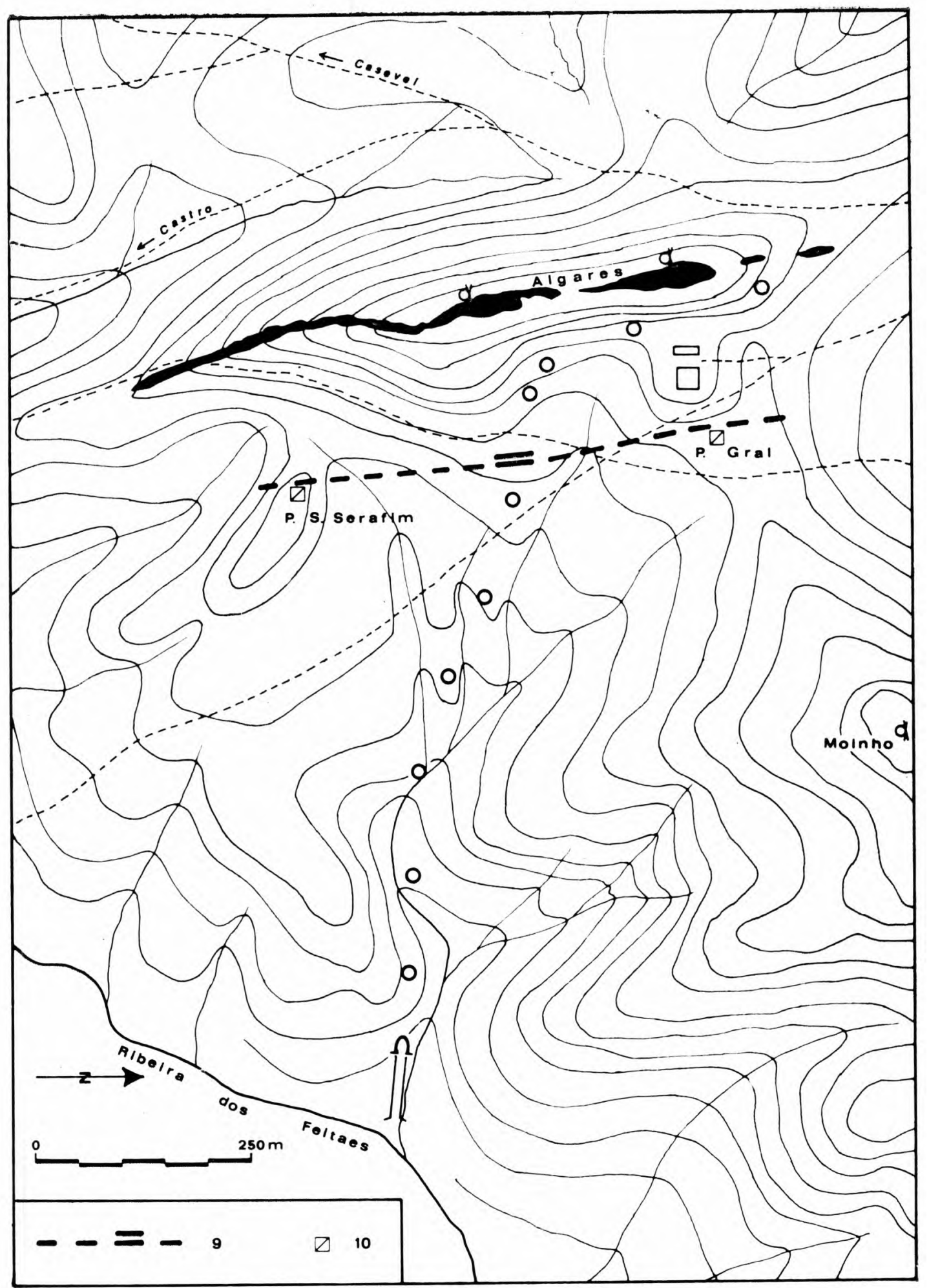

Fig. 25 


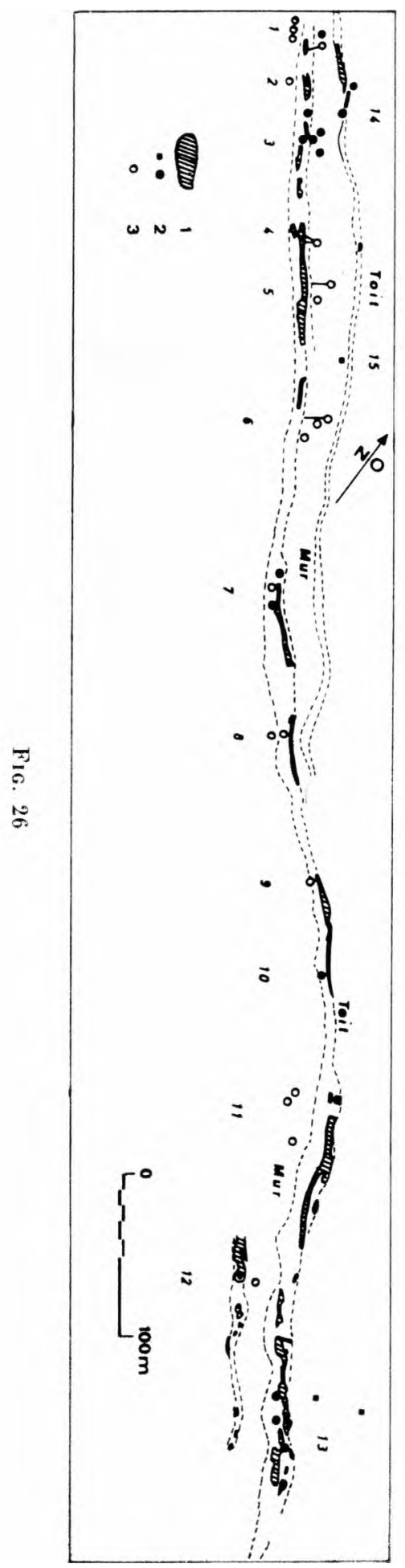




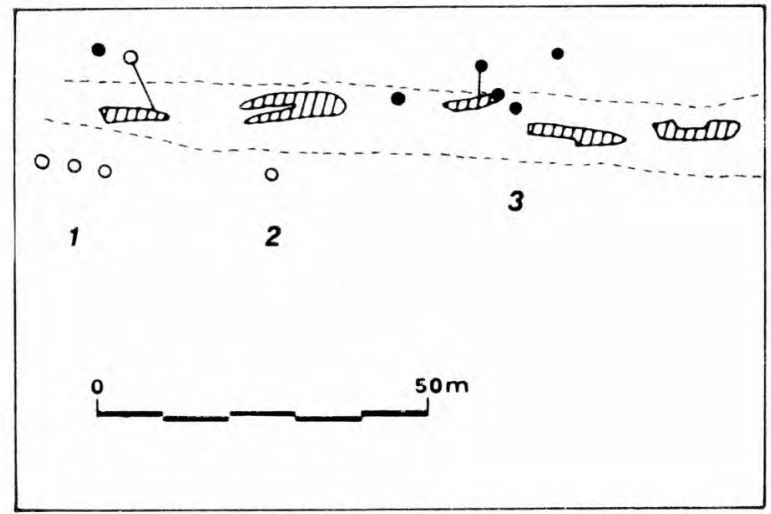

F16. 27 


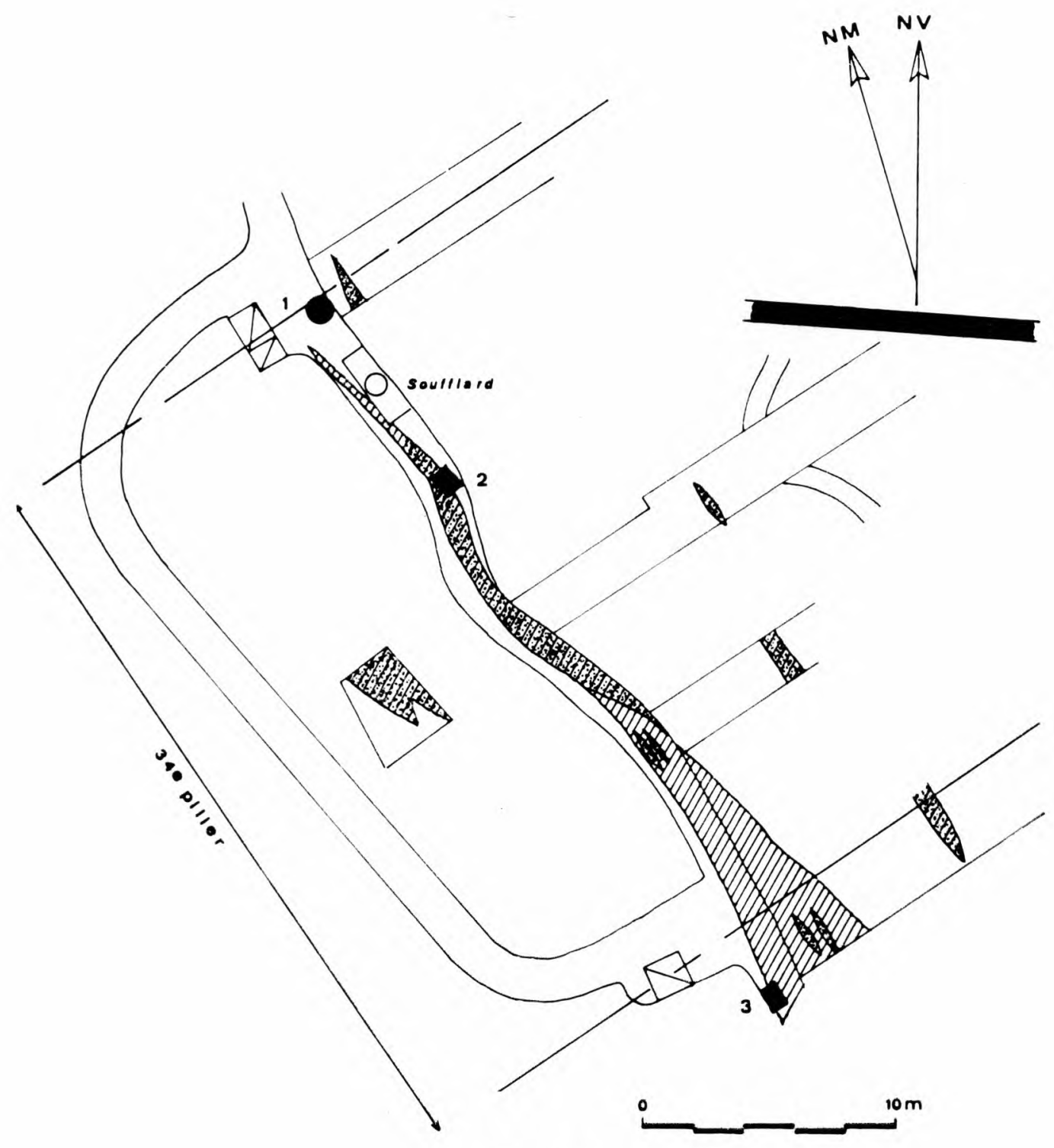

Fig. 28 


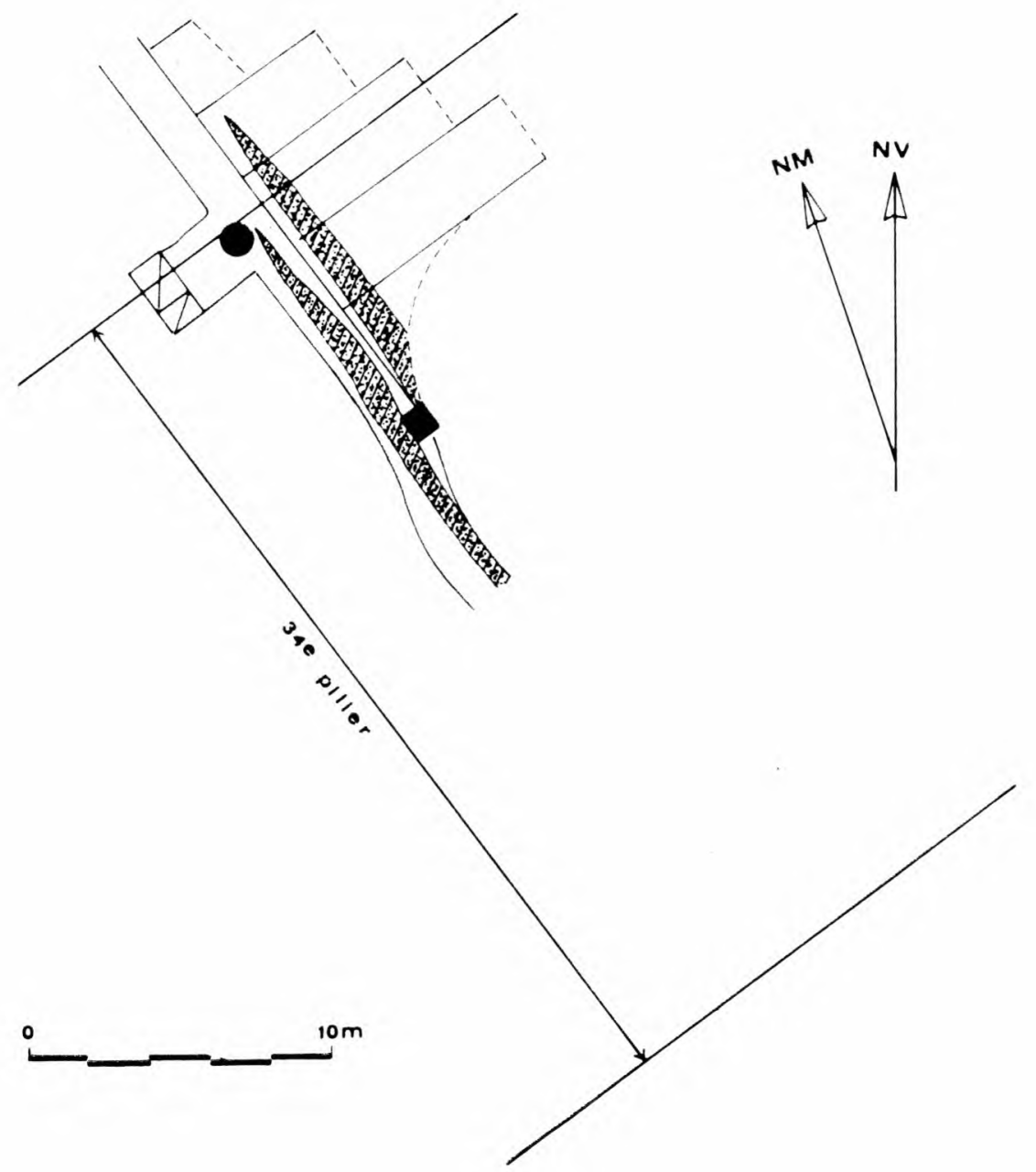

Fig. 29 


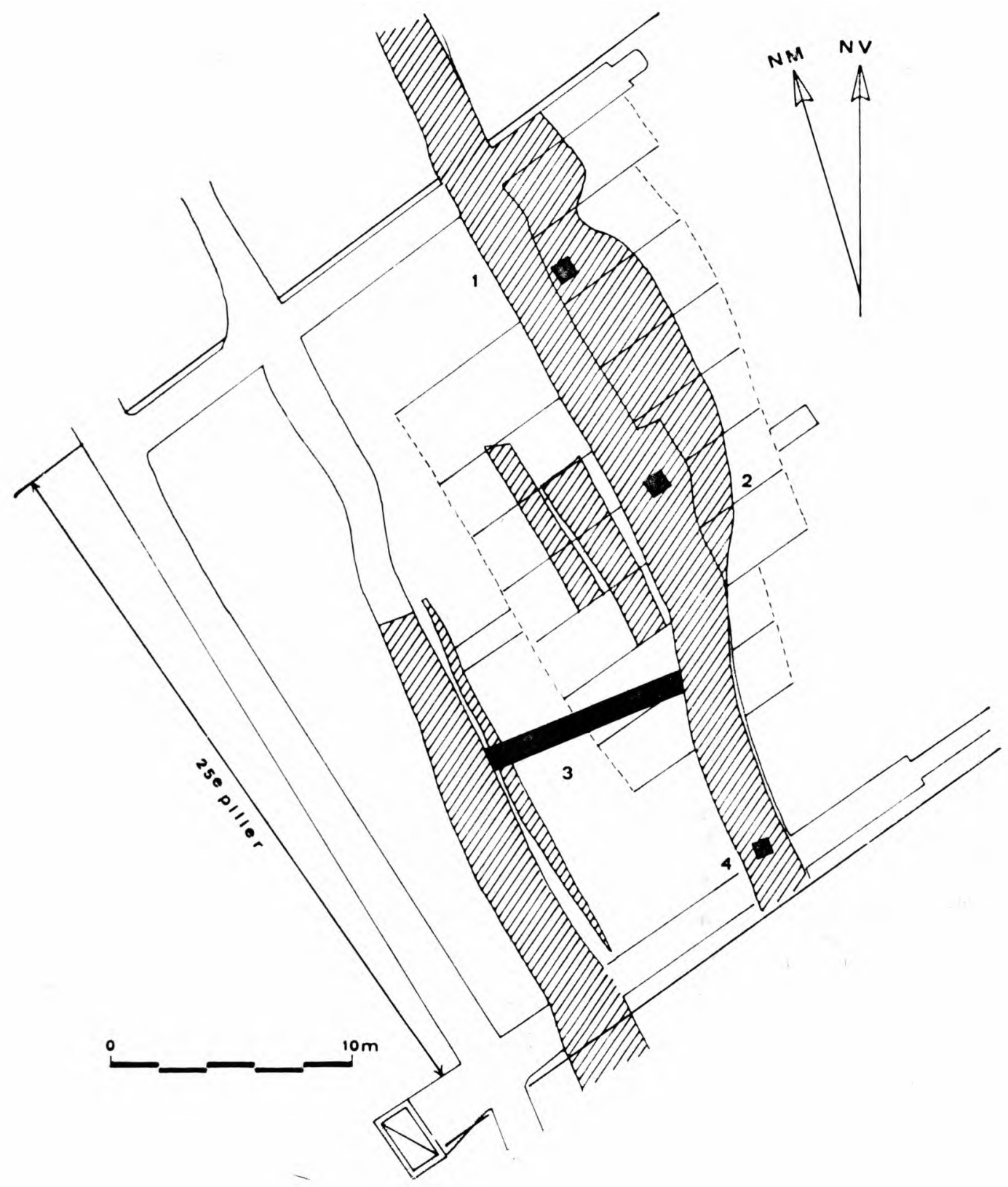

Fig. 30 


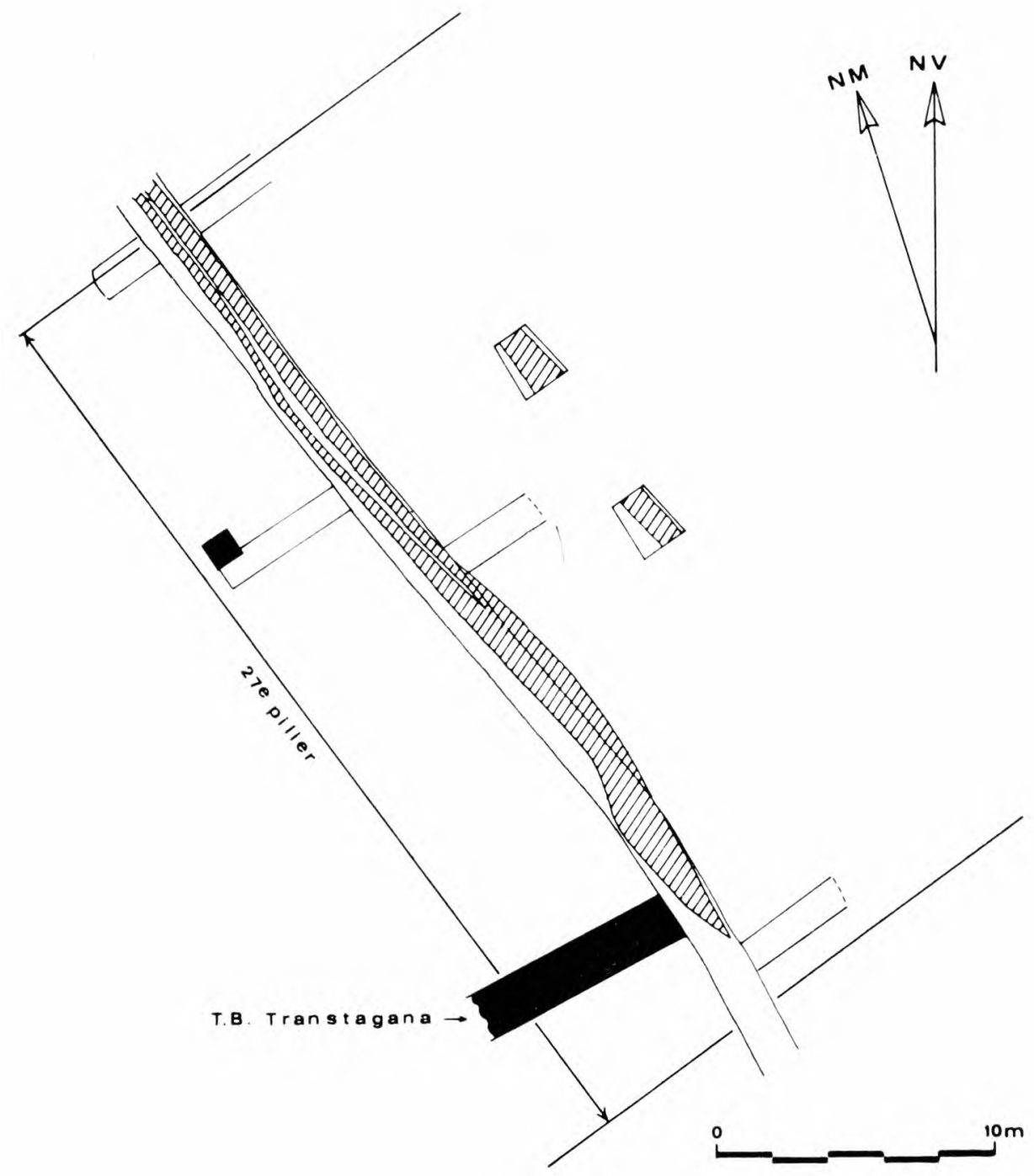

FIG. 31 


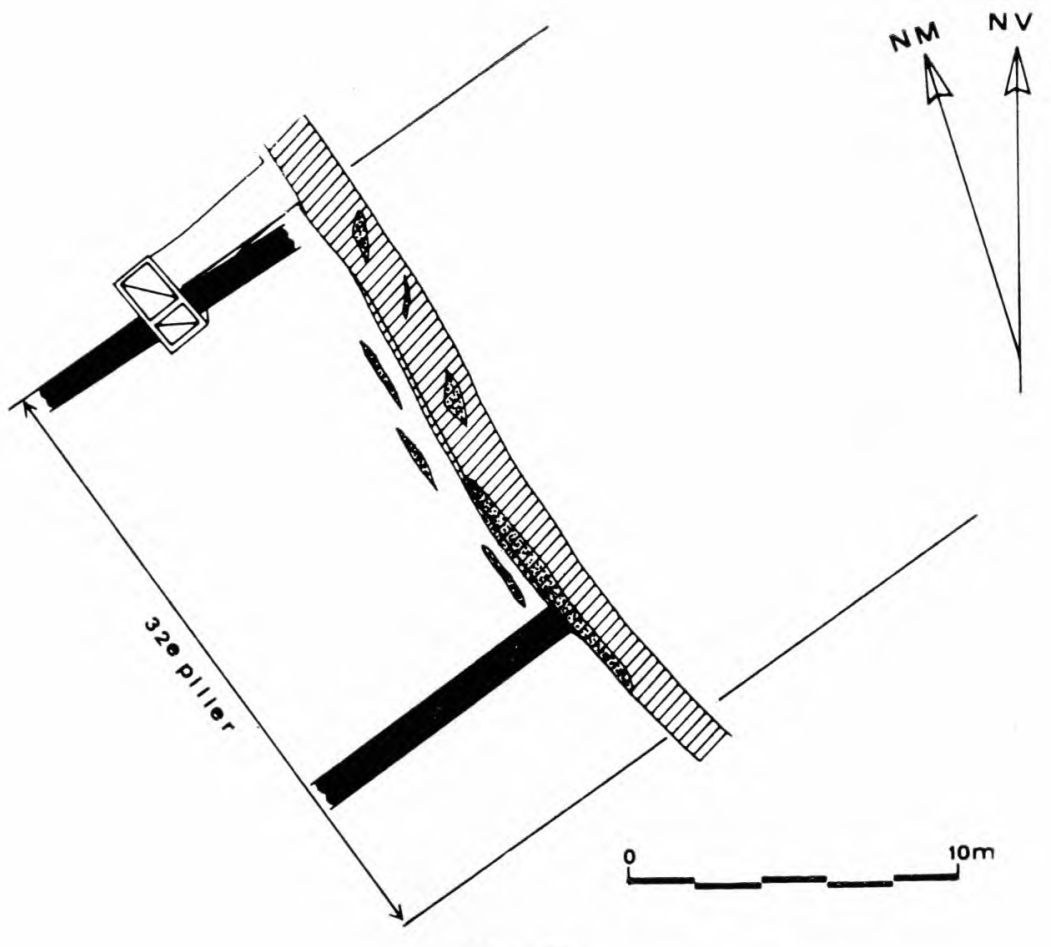

Fig. 32 

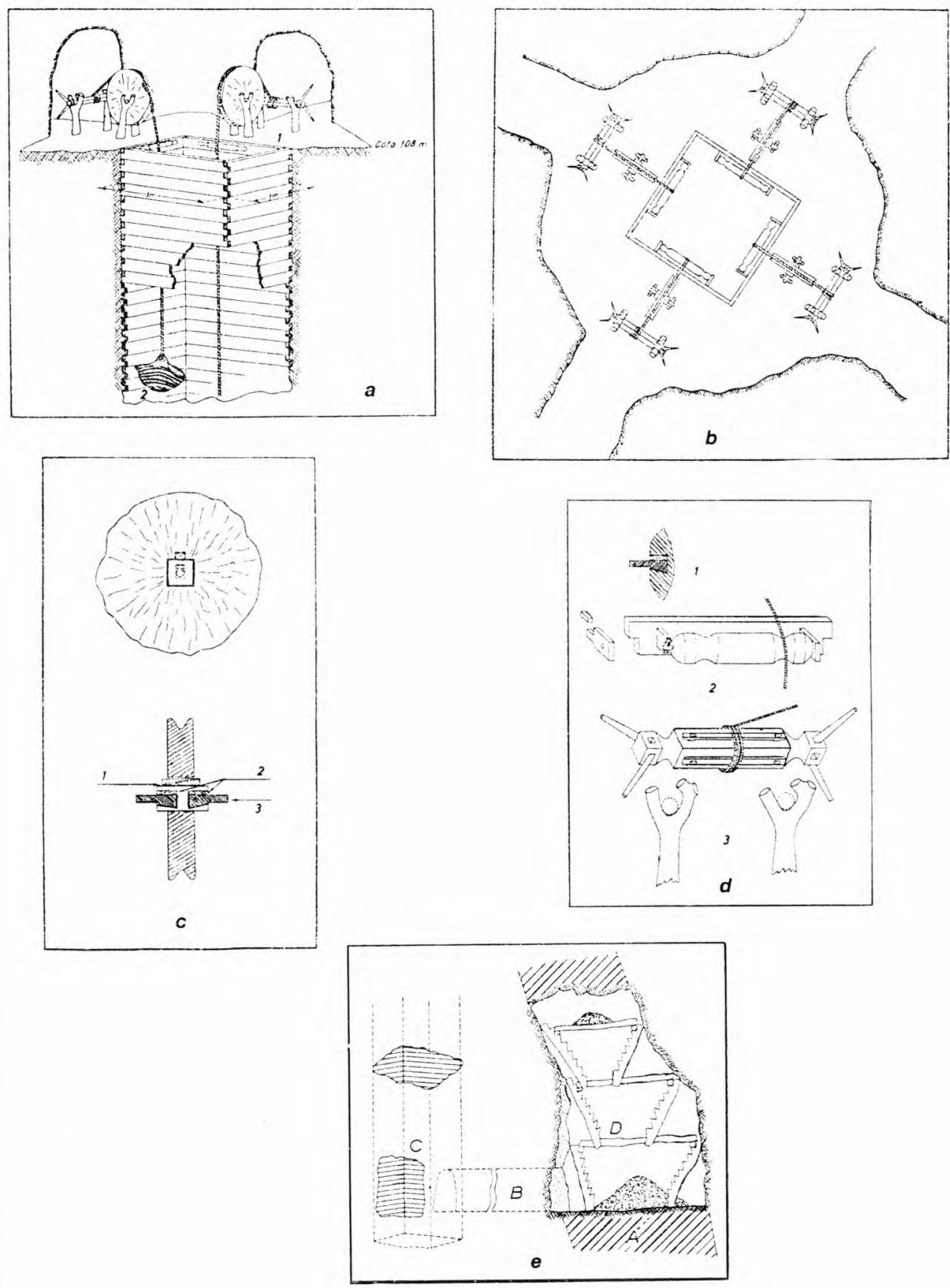

FIg. 33 

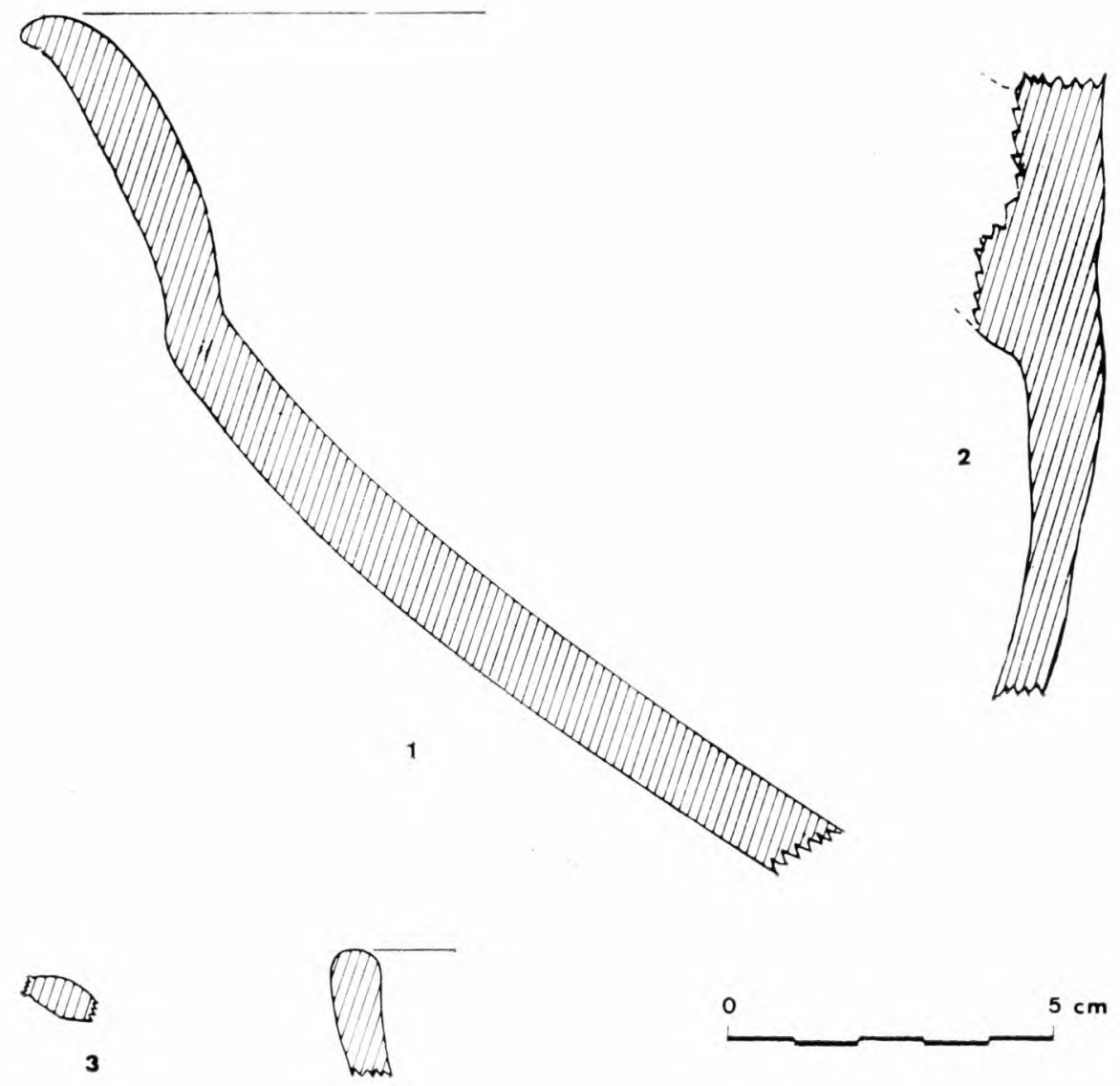

4

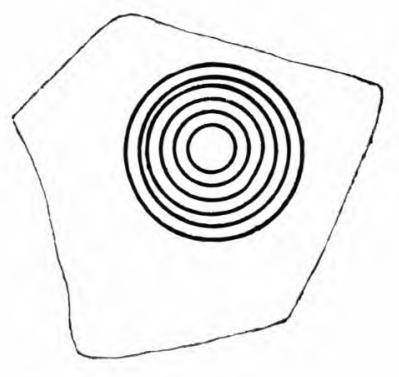

5

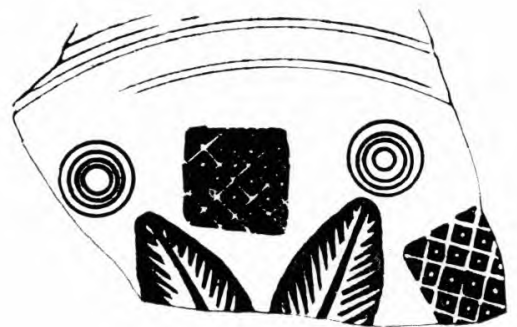

6

FIG. ${ }^{4}$ 


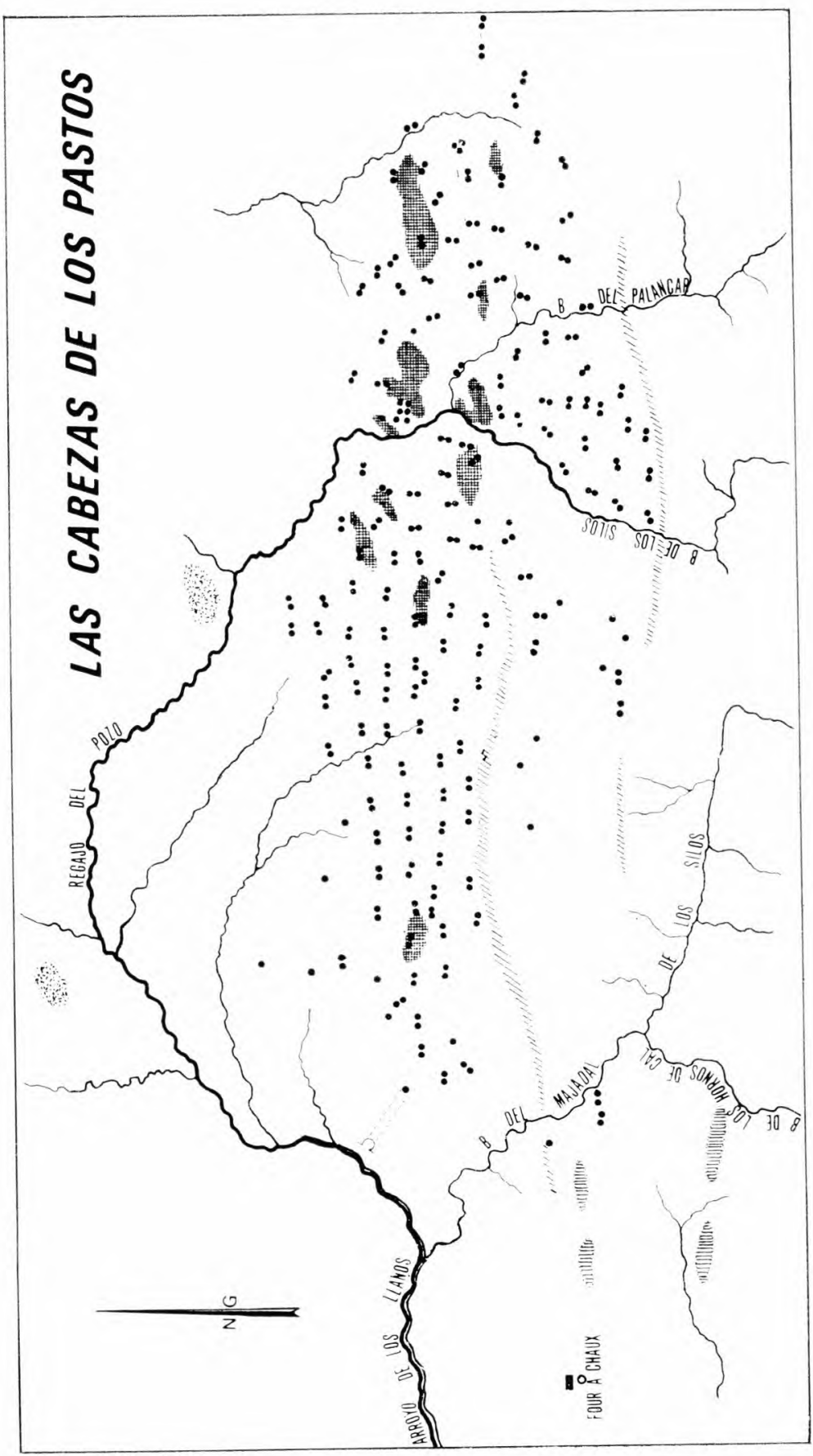




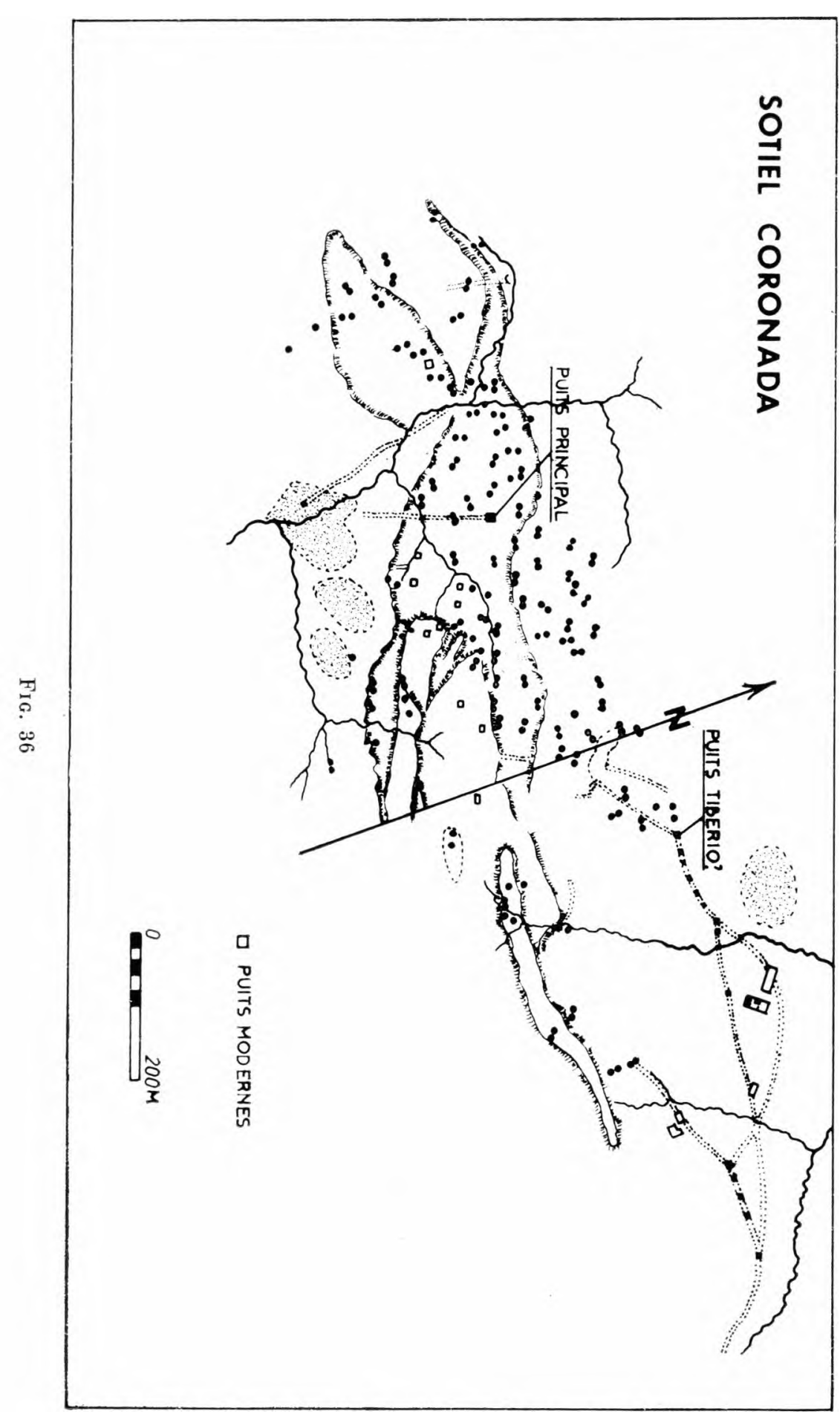

